sea. 3

JUL 221966 ENGINEERING DATA TRANSMITTAL

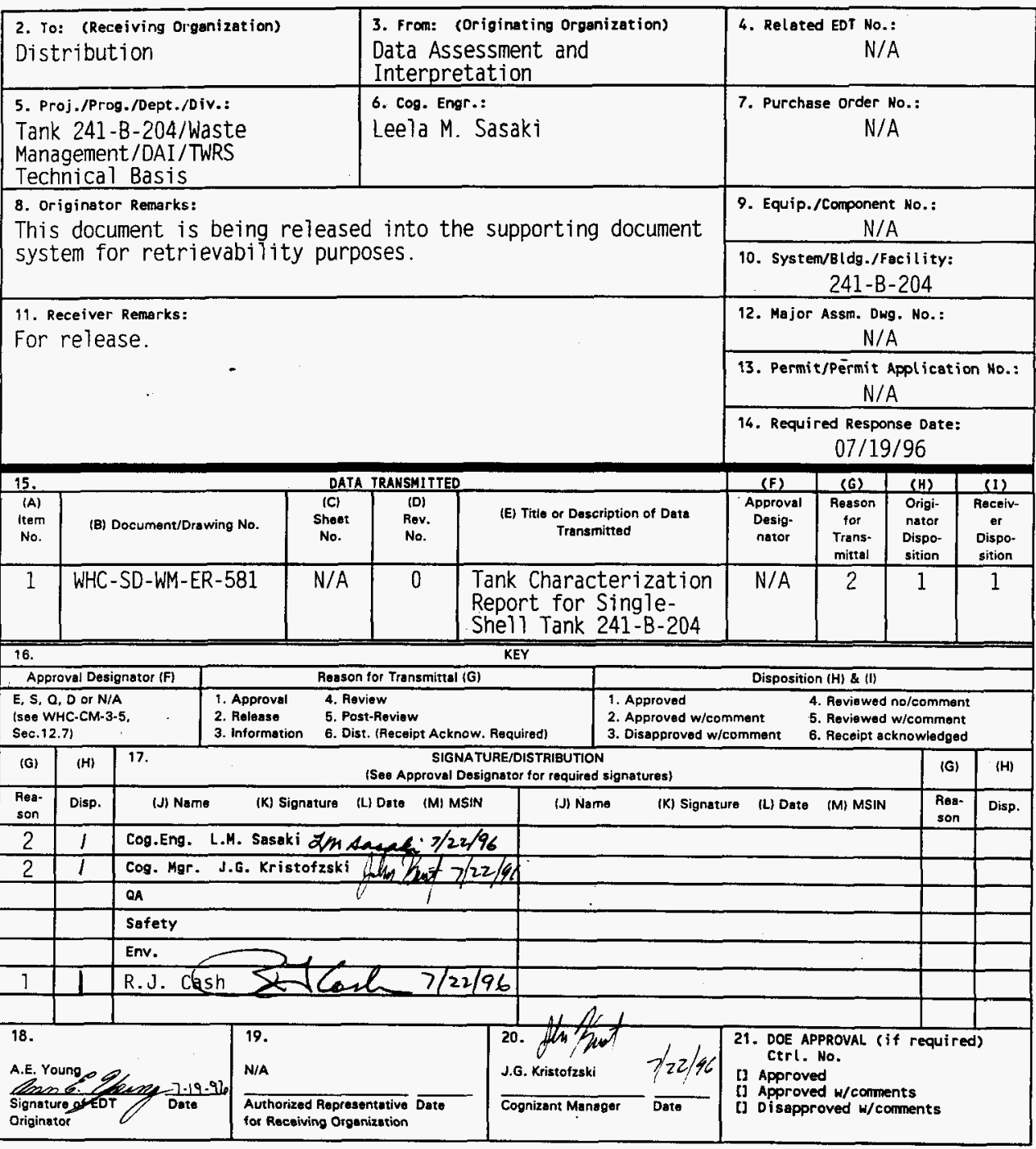

BD-7400-172-2 (04/94) GEF097 


\section{Tank Characterization Report for Single-Shell Tank 241-B-204}

Leela M. Sasaki

Westinghouse Hanford Company, Richland, WA 99352

U.S. Department of Energy Contract DE-AC06-87RL10930

$\begin{array}{lll}\text { EDT/ECN: } & \text { EDT }-617501 & \text { UC: } 2070 \\ \text { Org Code: } & 79400 & \text { Charge Code: N4G4D } \\ \text { B\&R Code: } & \text { EW 3120074 } & \text { Total Pages: } 254\end{array}$

Key words: Tank Characterization Report, TCR. Characterization, SingleShe11. SST, Tank 241-B-204. Tank B-204. B-204. B Farm. Milestone M-44-09

Abstract: This document summarizes the information on the historical uses, present status, and the sampling and analysis results of waste stored in Tank 241-B-204. This report supports the requirements of TriParty Agreement Mi lestone M-44-09.

TRADEMARK DISCLAIMER. Reference herein to any specific comercial product, process, or service by trade name, trademark, manufacturer, or otherwise, does not necessarily constitute or imply its endorsement, recommendation, or favoring by the United States Goverrment or any agency thereof or its contractors or subcontractors.

Printed in the United States of America. To obtain copies of this document, contact: WHC/BCS Document Control Services, P.O. Box 1970, Mailstop H6-08, Richland WA 99352, Phone (509) 372-2420; Fax (509) 376-4989.
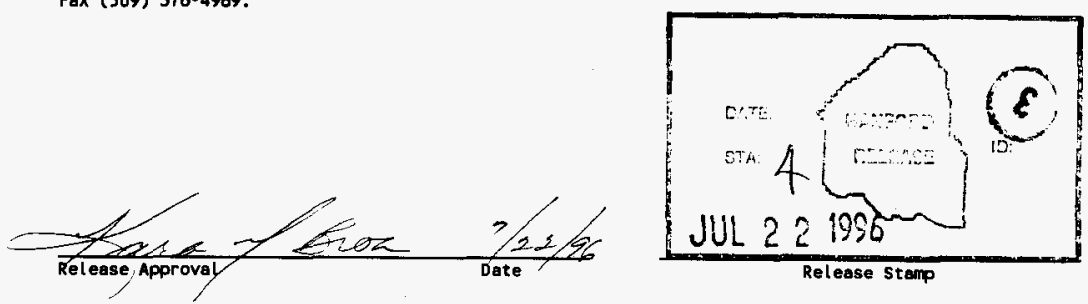

\section{Approved for Public Release}




\title{
Tank Characterization Report for Single-Shell Tank 241-B-204
}

\author{
L. M. Sasaki \\ J. G. Douglas
}

Westinghouse Hanford Company

R. H. Stephens

L. C. Amato

T. T. Tran

Los Alamos Technical Associates

Date Published

July 1996

Prepared for the U.S. Department of Energy

Assistant Secretary for Environmental Management

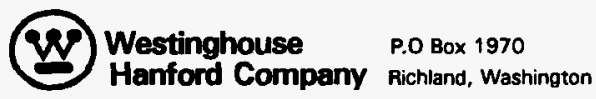

Manegement and Operations Contrector for the

U.S. Depertment of Energy under Contract DE-AC06-87RL10930

Approved for public release, distribution unlimited 


\section{EXECUTIVE SUMMARY}

This characterization report summarizes the available information on the historical uses, current status, and sampling and analysis results of waste stored in single-shell tank 241-B-204. This report supports the requirements of the Hanford Federal Facility Agreement and Consent Order, Milestone M-44-09 (Ecology et al. 1996).

Tank 241-B-204 is one of 16 tanks located in the Hanford 200 East Area B Tank Farm. The tank went into service in the second quarter of 1952 and received lanthanum fluoride waste (224) from the B Plant lanthanum fluoride process. The transfers continued for one more quarter. In the fourth quarter of 1952 and the first quarter of 1953 , the tank received B Plant flush waste. These were the last waste transfers into the tank. Other than removing $23 \mathrm{~kL}$ (6 kgal) of supernatant in 1974, no other transfer activity is recorded (Agnew et al. 1996). Tank 241-B-204 was removed from service in 1978, and it was declared an assumed leaker in 1984. Interim stabilization and intrusion prevention were completed in June 1984 and June 1985, respectively.

Table ES-1 describes tank 241-B-204, and Figure ES-1 shows a diagram of the tank. The tank has an operating capacity of $208 \mathrm{~kL}$ (55 kgal), and it currently contains an estimated $189 \mathrm{~kL}$ (50 kgal) of waste. The waste is estimated to be comprised of $185 \mathrm{~kL}$ (49 kgal) sludge, $4 \mathrm{~kL}$ (1 kgal) supernatant, and no saltcake or pumpable liquid. The sludge contains approximately $19 \mathrm{~kL}$ (5 kgal) of drainable interstitial liquid (Hanlon 1996). 
Table ES-1. Description and Status of Tank 241-B-204.

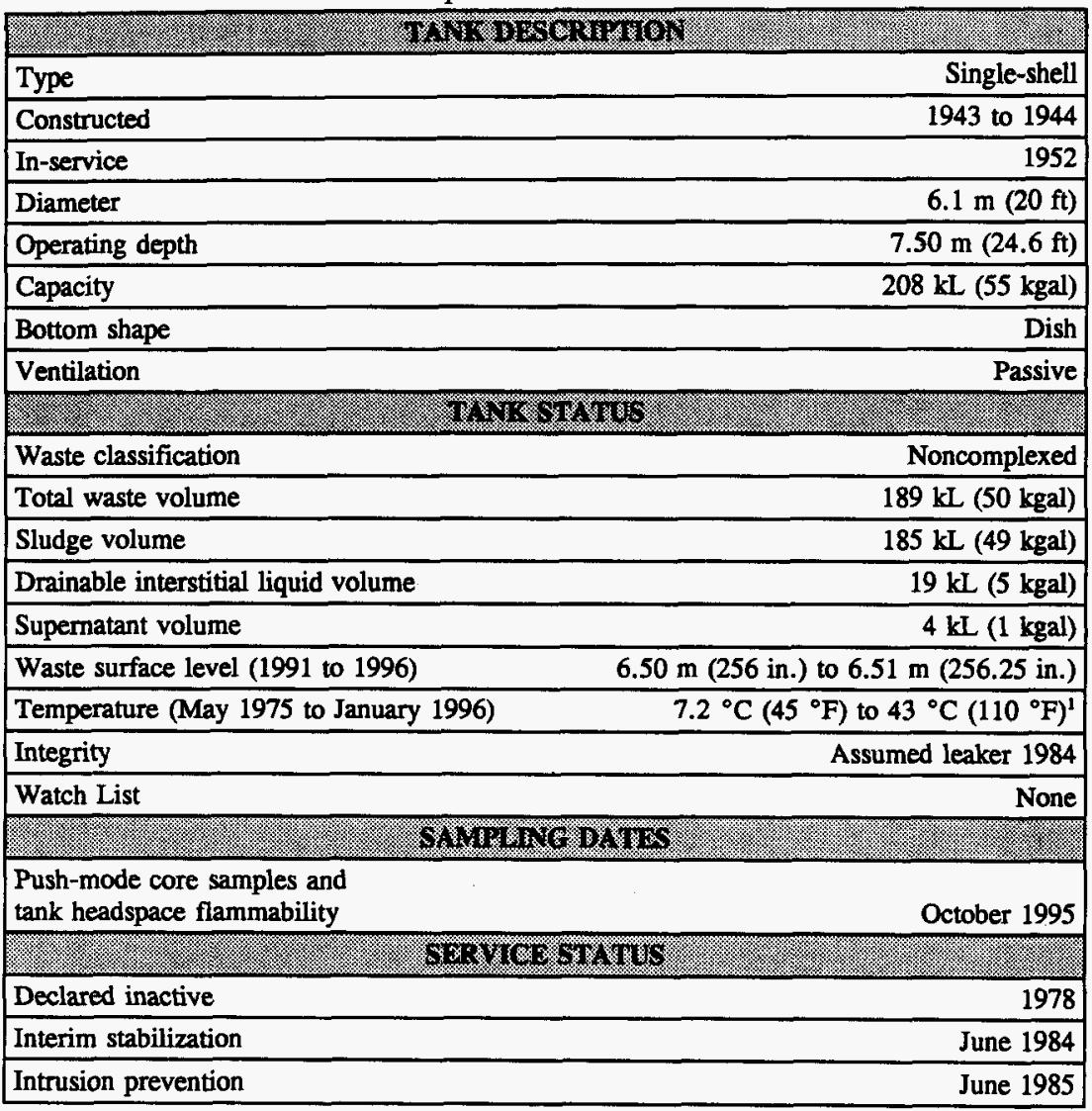

Note:

${ }^{1}$ The high temperature of $43^{\circ} \mathrm{C}$ is suspect. 
Figure ES-1. Profile of Tank 241-B-204.
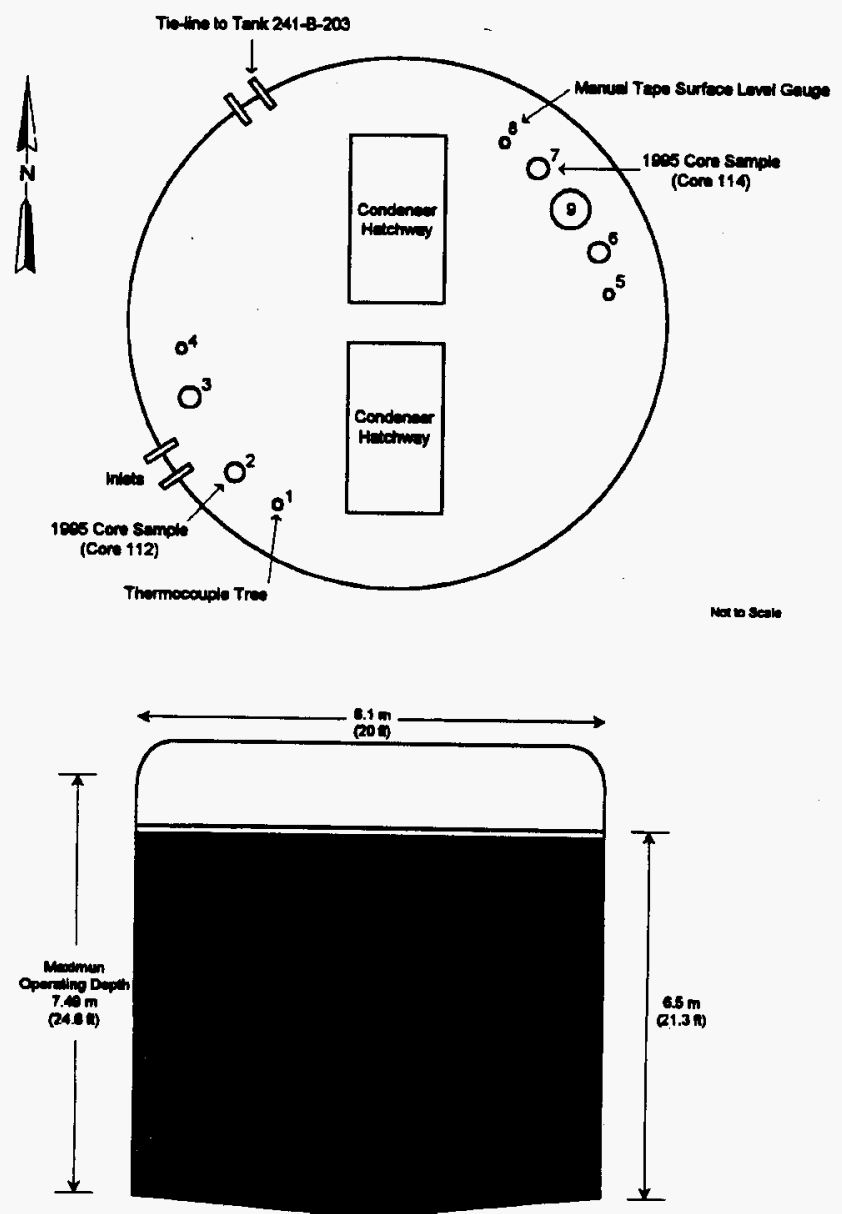

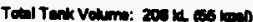

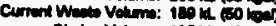

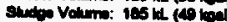

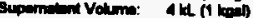

Not to scals 
This report summarizes the analysis of the core samples taken in October 1995. The sampling event was performed to satisfy the requirements of the Tank Safety Screening Data Quality Objective (Dukelow et al. 1995). The sampling and analyses were performed in accordance with the Tank 241-B-204 Push-Mode Sampling and Analysis Plan (Sasaki 1995). The sampling effort involved taking two push-mode core samples of the tank waste from widely-spaced risers. Core 112 was taken from riser 2, and core 114 was taken from riser 7.

The safety screening data quality objective (DQO) requires analyses for fuel content using differential scanning calorimetry (DSC), percent water by thermogravimetric analysis (TGA), total alpha activity through alpha proportional counting, and bulk density. The safety screening DQO also requires a determination of the flammability of the tank headspace gases. To satisfy this requirement, vapor samples were taken prior to core sampling, and the flammability was measured as a percent of the lower flammability limit using a combustible gas meter. The sampling and analysis plan (SAP) also required analyses for lithium and bromide to check for contamination of the samples by the hydrostatic head fluid (HHF) used during sampling operations. Additional results for other metals and anions were obtained as a result of these analyses.

No exothermic reactions were observed during the DSC analysis. The mean values for weight-percent water, determined by TGA, were 77.1 for sludge and 89.1 for drainable liquid. All total alpha activity results were well below the safety screening DQO notification limit of $41 \mu \mathrm{Ci} / \mathrm{g}$. Sludge bulk density results were $1.19 \mathrm{~g} / \mathrm{mL}$, while that of the drainable 
liquids was $1.05 \mathrm{~g} / \mathrm{mL}$. The flammability of tank $241-\mathrm{B}-204$ headspace was measured at zero percent of the lower flammability limit. The analytical results were well within the parameters listed in the safety screening DQO. Table ES-2 shows the average values for major analytes and analytes of interest.

The concentrations of individual radionuclides were measured in only one sample. Assuming that the sludge is homogeneous, a tank heat load of $<0.8 \mathrm{~W}(<3 \mathrm{Btu} / \mathrm{hr})$ was estimated. The historical tank content estimate (HTCE) heat load value was zero (Brevick et al. 1994a), and the heat load based on headspace temperature was $47.2 \mathrm{~W}(161 \mathrm{Btu} / \mathrm{hr})$ (Kummerer 1994). Both estimates were well below the $11,700 \mathrm{~W}(40,000 \mathrm{Btu} / \mathrm{hr})$ threshold differentiating high-heat from low-heat tanks (Bergmann 1991). The available temperature data for the tank support either heat load estimate. 
WHC-SD-WM-ER-581 Rev. 0

Table ES-2. Major Analytes and Analytes of Concern.

\begin{tabular}{|c|c|c|c|c|c|}
\hline 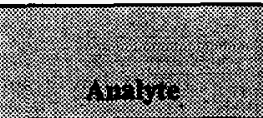 & 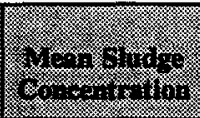 & 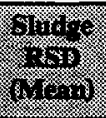 & 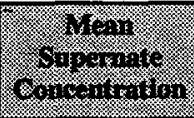 & $\begin{array}{l}\text { Supeninge } \\
\text { nesion: }\end{array}$ & 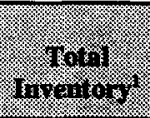 \\
\hline 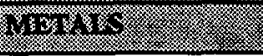 & $3-1,1 \%$ & 8 & $2.9 \mathrm{mi}$ & 3 & 76 \\
\hline Bismuth & 48,400 & 5.8 & $<15.1$ & $\mathrm{n} / \mathbf{a}$ & 10,700 \\
\hline Chromium & 3,240 & 5.7 & 154 & 2.7 & 715 \\
\hline Iron & 3,810 & 12.1 & $<7.53$ & $\mathbf{n} / \mathbf{a}$ & 839 \\
\hline Lanthanum & 10,400 & 2.6 & $<7.53$ & $n / a$ & 2,290 \\
\hline Manganese & 14,800 & 2.7 & $<1.51$ & $n / a$ & 3,270 \\
\hline Potassium & 5,780 & 1.8 & 6,110 & 4.6 & 1,290 \\
\hline Sodium & 26,200 & 2.3 & 28,000 & 2.2 & 5,880 \\
\hline Haros. & 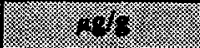 & 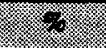 & 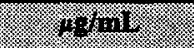 & (3) & 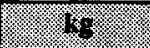 \\
\hline Fluoride & 7,080 & 9.5 & 6,240 & 6.0 & 1,580 \\
\hline Nitrate & 52,900 & 7.4 & 45,900 & 3.8 & 11,800 \\
\hline Phosphate & 3,620 & 8.8 & 2,060 & 12.4 & 805 \\
\hline 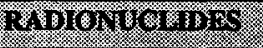 & 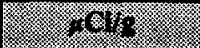 & 8 & $26 \operatorname{lng}$ & $\sqrt{2}$ & (2) \\
\hline Total alpha & 0.264 & 7.2 & 9.98E-05 & 8.2 & 58.1 \\
\hline 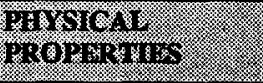 & S.ling & 4 & 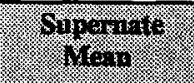 & \% & \\
\hline Weight percent water & $77.1 \%$ & 0.9 & $89.1 \%$ & 0.2 & 173,000 \\
\hline Density & $1.19 \mathrm{~g} / \mathrm{mL}$ & 2.0 & $1.05 \mathrm{~g} / \mathrm{mL}$ & 0.5 & $n / a$ \\
\hline
\end{tabular}

Notes:

RSD (Mean) (\%) = $100 \times$ standard deviation of the mean $\div$ mean value

$\mathbf{n} / \mathbf{a}=$ not applicable

'Total inventory $=$ total sludge mass $(220,000 \mathrm{~kg})$ plus total supernate mass $(3,970 \mathrm{~kg})$. 


\section{CONTENTS}

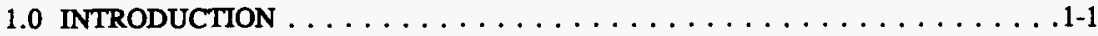

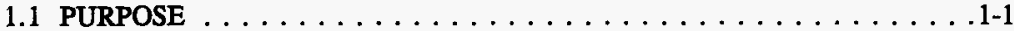

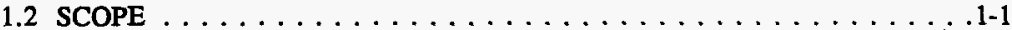

2.0 HISTORICAL TANK INFORMATION $\ldots \ldots \ldots \ldots \ldots \ldots \ldots \ldots \ldots \ldots$ 2-1

2.1 TANK STATUS $\ldots \ldots \ldots \ldots \ldots \ldots \ldots \ldots \ldots \ldots \ldots .2-1$

2.2 TANK DESIGN AND BACKGROUND $\ldots \ldots \ldots \ldots \ldots \ldots \ldots \ldots .2-2$

2.3 PROCESS KNOWLEDGE $\ldots \ldots \ldots \ldots \ldots \ldots \ldots \ldots \ldots \ldots . . \ldots \ldots$

2.3.1 Waste Transfer History . . . . . . . . . . . . . 2-6

2.3.2 Historical Estimation of Tank Contents . . . . . . . 2-6

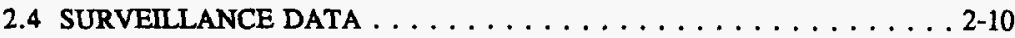

2.4.1 Surface Level . . . . . . . . . . . . . . . . . 2-10

2.4.2 Internal Tank Temperatures . . . . . . . . . . 2-10

2.4.3 Tank 241-B-204 Photograph . . . . . . . . . . . . 2-13

3.0 TANK SAMPLING OVERVIEW $\ldots \ldots \ldots \ldots \ldots \ldots \ldots \ldots \ldots$ 3-1

3.1 DESCRIPTION OF SAMPLING EVENT $\ldots \ldots \ldots \ldots \ldots \ldots \ldots \ldots$ 3-1

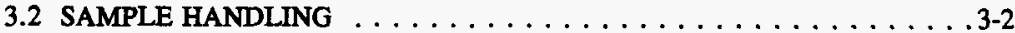

3.3 SAMPLE ANALYSIS $\ldots \ldots \ldots \ldots \ldots \ldots \ldots \ldots \ldots . \ldots \ldots$. 2

3.4 DESCRIPTION OF HISTORICAL SAMPLING EVENT $\ldots \ldots \ldots \ldots$. $\ldots$

4.0 ANALYTICAL RESULTS $\ldots \ldots \ldots \ldots \ldots \ldots \ldots \ldots \ldots \ldots .1$

4.1 DATA PRESENTATION $\ldots \ldots \ldots \ldots \ldots \ldots \ldots \ldots \ldots \ldots$. . . . . . .

4.1.1 Chemical Data Summary . . . . . . . . . . . . . . 4-1

4.1.2 Physical Data Summary . . . . . . . . . . . . 4-5

4.1.3 Tank Headspace Flammability . . . . . . . . . . . . . 4-7

4.1.4 Hydrostatic Head Fluid Contamination Check . . . . . . . . . 4-7

5.0 INTERPRETATION OF CHARACTERIZATION RESULTS $\ldots \ldots \ldots \ldots$. . .

5.1 ASSESSMENT OF SAMPLING AND ANALYTICAL RESULTS $\ldots \ldots$ 5-1

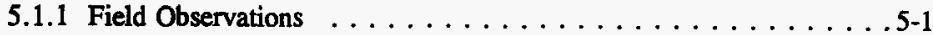

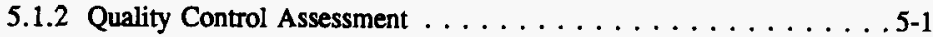

5.1 .3 Data Consistency Checks . . . . . . . . . . . . 5-2

5.2 COMPARISON OF HISTORICAL WITH ANALYTICAL RESULTS . . . 5-5

5.3 TANK WASTE PROFILE $\ldots \ldots \ldots \ldots \ldots \ldots \ldots \ldots \ldots . \ldots . . \ldots$

5.4 COMPARISON OF TRANSFER HISTORY WITH ANALYTICAL RESULTS $\ldots \ldots \ldots \ldots \ldots \ldots \ldots \ldots \ldots$. . . . . . . . . .

5.5 EVALUATION OF PROGRAM REQUIREMENTS $\ldots \ldots \ldots \ldots \ldots \ldots$ 5-13 5.5 .1 Safety Evaluation $\ldots \ldots \ldots \ldots \ldots \ldots \ldots \ldots \ldots \ldots \ldots \ldots \ldots$ 
WHC-SD-WM-ER-581 Rev. 0

CONTENTS (Cont'd)

6.0 CONCLUSIONS AND RECOMMENDATIONS $\ldots \ldots \ldots \ldots \ldots \ldots$. . . . . .

7.0 REFERENCES $\ldots \ldots \ldots \ldots \ldots \ldots \ldots \ldots \ldots \ldots \ldots \ldots \ldots \ldots \ldots \ldots \ldots .1$

\section{APPENDICES}

A SAMPLE INFORMATION FOR 1995 CORE SAMPLING $\ldots \ldots \ldots \ldots$ A-1

B ANALYTICAL RESULTS FROM 1995 CORE SAMPLING . . . . . . . . B-1

C RESULTS OF HYDROSTATIC HEAD FLUID CONTAMINATION CHECK FOR SINGLE-SHELL TANK $241-B-204 \ldots \ldots \ldots \ldots \ldots \ldots \ldots \ldots$ C-1

D HISTORICAL SAMPLING RESULTS $\ldots \ldots \ldots \ldots \ldots \ldots$ D-1

\section{LIST OF FIGURES}

2-1 Riser Configuration for Tank 241-B-204 . . . . . . . . . . 2-3

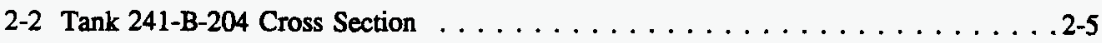

2-3 Tank Layer Model for Tank 241-B-204 . . . . . . . . . . . . . . . . 2-7

2-4 Tank 241-B-204 Level History . . . . . . . . . . . . . . 2-11

2-5 Tank 241-B-204 Weekly High Temperature Plot . . . . . . . . . . . . 2-12 2-6 Tank 241-B-204 In-Tank Photograph. . . . . . . . . . . . . . . 2-15 


\section{LIST OF TABLES}

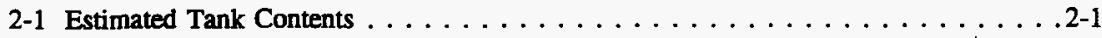

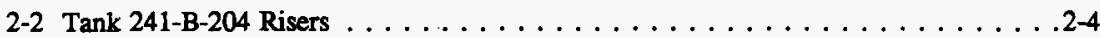

2-3 Summary of Tank 241-B-204 Waste Transfer History $\ldots \ldots \ldots \ldots \ldots \ldots$. . . . . . .

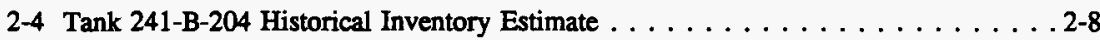

3-1 Integrated Data Quality Objective Requirements for Tank 241-B-204 . . . . . . . 3-1

4-1 Anaiytical Data Presentation Tables $\ldots \ldots \ldots \ldots \ldots \ldots \ldots \ldots$ 4-1

4-2 Chemical Data Summary for Tank $241-B-204 \ldots \ldots \ldots \ldots \ldots \ldots$. $\ldots \ldots \ldots$. $\ldots \ldots$

5-1 Cation Mass and Charge Data $\ldots \ldots \ldots \ldots \ldots \ldots \ldots \ldots \ldots \ldots$

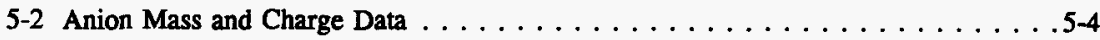

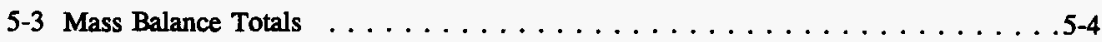

5-4 Comparisons of Sludge Data from the 1978 and 1995 Sampling Events for Tank $241-B-204 \ldots \ldots \ldots \ldots \ldots \ldots \ldots \ldots \ldots$. . . . . . . . . . . . .

5-5 Comparison of B-200 Series Tank Compositions $\ldots \ldots \ldots \ldots \ldots \ldots$ 5-8

5-6 Comparison of HTCE Predictions with the 1995 Analytical Results for Tank 241-B-204 . . . . . . . . . . . . . . . . . . . . 5-11

5-7 Safety Screening Data Quality Objective Decision Variables and Criteria . . . . 5-14 
WHC-SD-WM-ER-581 Rev. 0

This page intentionally left blank. 


\section{LIST OF TERMS}

224

ANOVA

Btu/hr

Ci

$\mathrm{Ci} / \mathrm{g}$

DQO

DSC

DL

$\mathrm{ft}$

gal

g

g/L

$\mathrm{g} / \mathrm{mL}$

GEA

HDW

HHF

HTCE

in.

IC

ICP

$\mathrm{J} / \mathrm{g}$

$\mathrm{kg}$

kgal

$\mathrm{kL}$

L

LL

m

$\mathrm{mg}$

$\mathrm{mL}$

$\mathrm{mol} / \mathrm{L}$

ppm

RPD

RSD

SAP

TLM

TGA

W

WHC

wt\%

${ }^{\circ} \mathrm{C}$

${ }^{\circ} \mathrm{F}$

$\mu \mathrm{Ci} / \mathrm{g}$ lanthanum fluoride waste

analysis of variance

British thermal units per hour

curies

curies per gram

data quality objective

differential scanning calorimetry

drainable liquid

feet

gallons

grams

grams per liter

grams per milliliter

gamma energy analysis

Hanford Defined Wastes

hydrostatic head fluid

historical tank content estimate

inches

ion chromatography

inductively coupled plasma spectroscopy

joules per gram

kilograms

kilogallons

kiloliters

liters

liner liquid

meters

milligrams

milliliters

moles per liter

parts per million

relative percent difference

relative standard deviation

sampling and analysis plan

Tank Layer Model

thermogravimetric analysis

watts

Westinghouse Hanford Company

weight percent

degrees Celsius

degrees Fahrenheit

microcuries per gram 
WHC-SD-WM-ER-581 Rev. 0

\section{LIST OF TERMS (Continued)}

$\mu \mathrm{Ci} / \mathrm{mL} \quad$ microcuries per milliliter

$\mu \mathrm{eq} / \mathrm{g} \quad$ microequivalents per gram

$\mu \mathrm{g} / \mathrm{g} \quad$ micrograms per gram

$\mu \mathrm{g} / \mathrm{mL} \quad$ micrograms per milliliter 


\subsection{INTRODUCTION}

This tank characterization report presents an overview of single-shell tank 241-B-204 and its waste contents. It provides estimated concentrations and inventories for waste components based on the latest sampling and analysis activities in combination with background tank information. The characterization of tank 241-B-204 is based on the results of a core sampling event in October 1995. For information purposes, results from a 1978 sampling event are also included. Other 241-B-200 series tanks contain waste similar to that in tank 241-B-204. For additional information, refer to Heasler et al. (1994) for tank 241-B-201 and Dougherty and Tran (1995) for tank 241-B-202.

Tank 241-B-204 was removed from service in 1978. Interim stabilization was completed in June 1984, and intrusion prevention was completed in June 1985; therefore, the composition of the waste should not change appreciably until pretreatment and retrieval activities commence. The analyte concentrations reported in this document reflect the best composition estimates of the waste based on the available analytical data and historical models. This report supports the requirements of the Hanford Federal Facility Agreement and Consent Order, Milestone M-44-09 (Ecology et al. 1996).

\subsection{PURPOSE}

The purpose of this report is to summarize the information about the use and contents of tank 241-B-204. When possible, this information will be used to assess issues associated with safety, operations, environmental, and process development activities. This report also serves as a reference point for more detailed information about tank 241-B-204.

\subsection{SCOPE}

The October 1995 core sampling event for tank 241-B-204 supported the evaluation of tank waste according to the Tank Safety Screening Data Quality Objective (Dukelow et al. 1995). Safety screening analyses were performed on the two core samples as directed in the Tank 241-B-204 Push Mode Core Sampling and Analysis Plan (Sasaki 1995). These analyses were differential scanning calorimetry (DSC) to evaluate fuel level and energetics, thermogravimetric analysis (TGA) to determine moisture content, total alpha activity analysis to evaluate criticality potential, and bulk density. The flammability of the tank headspace gases was also measured as required by the safety screening DQO. The SAP also required analyses for lithium by inductively coupled plasma spectroscopy (ICP) and bromide by ion chromatography (IC) to account for any hydrostatic head fluid (HHF) contamination during the core sampling operation. Results for additional analytes were obtained during the ICP and IC analyses and are included in this report. Gamma energy analyses (GEA) and ${ }^{90} \mathrm{Sr}$ analysis were performed on one segment to gather information for the disposal of laboratory waste (Sasaki 1996b). 
WHC-SD-WM-ER-581 Rev. 0

This page intentionally left blank. 


\subsection{HISTORICAL TANK INFORMATION}

This section describes tank 241-B-204 based on historical information. The first part details the current condition of the tank. It includes discussions of tank design, transfer history, process sources contributing to the tank waste, and an estimate of the current contents based on the process history. It also includes events that may be related to tank safety issues, such as potentially hazardous tank contents or off-normal operating temperatures. The final part summarizes available surveillance data for the tank. Solid and liquid level data are used to determine tank integrity (leaks) and to provide clues to internal activity in the solid layers of the tank. Temperature data are provided to evaluate the heat generating characteristics of the waste.

\subsection{TANK STATUS}

As of January 31, 1996, tank 241-B-204 contained an estimated $189 \mathrm{~kL}$ (50 kgal) of waste classified as noncomplexed (Hanlon 1996). Liquid volume was determined photographically. Solids volume was determined using a manual tape surface level gauge. The estimated amounts of the waste phases in the tank are shown in Table 2-1.

Table 2-1. Estimated Tank Contents. ${ }^{1}$

\begin{tabular}{|c|c|c|}
\hline 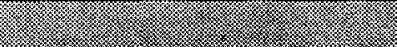 & 28 & (8) \\
\hline 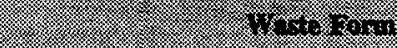 & (-2) & 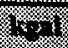 \\
\hline Total waste & 189 & 50 \\
\hline Supernatant liquid & 4 & 1 \\
\hline Sludge & 185 & 49 \\
\hline Saltcake & 0 & 0 \\
\hline Drainable interstitial liquid & 19 & 5 \\
\hline Drainable liquid remaining & 23 & 6 \\
\hline Pumpable liquid remaining & 0 & $\mathbf{0}$ \\
\hline
\end{tabular}

Note:

${ }^{1} H a n l o n$ (1996) 
Tank 241-B-204 was declared an assumed a leaker in 1984 with a leak volume of approximately $1,500 \mathrm{~L}$ (400 gal). Interim stabilization was completed in June 1984, and intrusion prevention was completed in June 1985. Tank 241-B-204 is a low heat load tank that is passively ventilated. It is not on any Watch Lists. All monitoring systems were in compliance with documented standards as of January 31, 1996 (Hanlon 1996).

\subsection{TANK DESIGN AND BACKGROUND}

The 241-B Tank Farm is a first generation tank farm. Built between 1943 and 1944, it consists of $122,006 \mathrm{~kL}$ (530 kgal) and four $208 \mathrm{~kL}(55 \mathrm{kgal})$ single-shell tanks (Leach and Stahl 1993). The tanks were designed for nonboiling waste with a maximum fluid temperature of $104^{\circ} \mathrm{C}\left(220^{\circ} \mathrm{F}\right)$. As with all first generation tank farms, equipment to monitor the waste is sparse. A typical B Farm 208-kL (55-kgal) tank contains risers that provide surface level access to the underground tank. The risers range from $10 \mathrm{~cm}(4 \mathrm{in.})$ to $30 \mathrm{~cm}$ (12 in.).

Tank 241-B-204 is constructed of 0.3-m (1-ft) thick reinforced concrete with a .64-cm (0.25-in.)-thick mild carbon-steel liner on the bottom and sides and a 30-cm (12-in.) thick flat concrete top (Brevick 1994a). Tank 241-B-204 is equipped with nine risers through the top of the tank. The risers range in diameter from $10 \mathrm{~cm}(4 \mathrm{in}$.) to $1.1 \mathrm{~m}(3.5 \mathrm{ft})$. The carbon-steel liner has a $7.49 \mathrm{~m}(24.6 \mathrm{ft})$ operating depth, is $6.1 \mathrm{~m}(20 \mathrm{ft})$ in diameter, and has a $15-\mathrm{cm}(6-\mathrm{in}$.) deep-dished bottom with a $0.9 \mathrm{~m}(3 \mathrm{ft})$ radius knuckle. The tank is set on a reinforced concrete foundation. At the time of construction, the tank was waterproofed on the sides and top with tar and gunite. The tank is covered with approximately $3.5 \mathrm{~m}$ $(11.5 \mathrm{ft})$ of overburden.

The four 200-series tanks on the B tank farm are at the same elevation. Tank 241-B-204 is connected to $\tan \mathrm{k} 241-\mathrm{B}-203$ by a $7.6-\mathrm{cm}$ (3-in.) diameter line that enables waste transfers from one tank to the other.

Figure 2-1 shows the riser configuration. Table 2-2 lists tank 241-B-204 risers, their diameter, and a description of each. Figure 2-2 shows a cross section of the tank with the approximate waste level and a schematic of the tank equipment. Tank 241-B-204 has a construction manhole and eight grade-level risers (Alstad 1993). A thermocouple tree in riser 1 monitors the tank waste temperature, and a manual tape through riser 8 measures the surface level. Risers 2, 3, 6 and 7 (30 cm [12 in.] in diameter) and risers 4 and $5(10 \mathrm{~cm}$ [4 in.] in diameter) access the two sides of the tank (Lipnicki 1995). 
Figure 2-1. Riser Configuration for Tank 241-B-204.

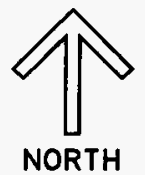

$$
\begin{array}{r}
210 \mathrm{~kL} \\
\text { [55kgal] }
\end{array}
$$

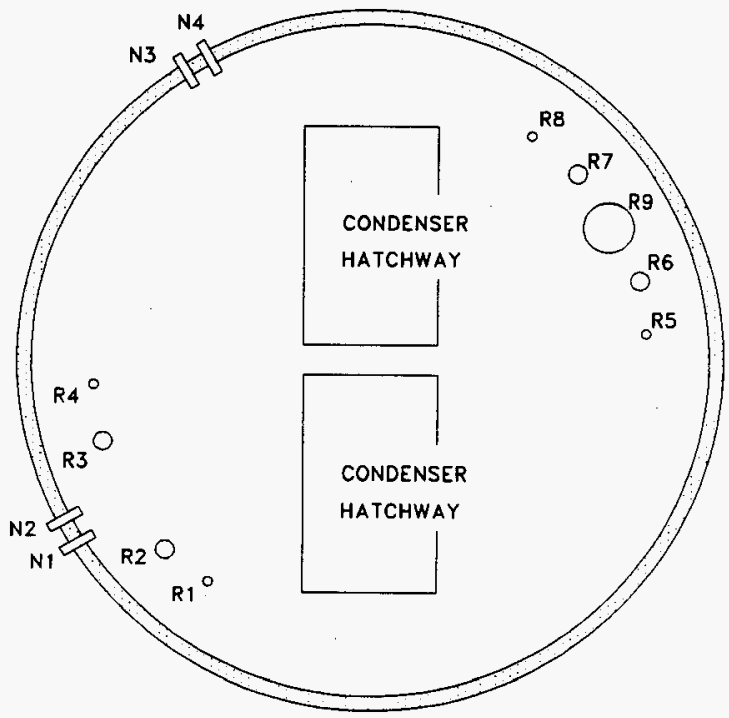

204203202201

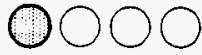

(112) (109 103

(111) (108 (105)

(110) (107) (101)

KEY PLAN 
WHC-SD-WM-ER-581 Rev. 0

Table 2-2. Tank 241-B-204 Risers. ${ }^{1,2}$

\begin{tabular}{|c|c|c|}
\hline 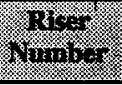 & 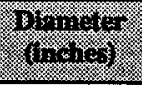 & Bescrintion ind Conments. \\
\hline R1 & 4 & Thermocouple tree \\
\hline $\mathbf{R} \mathbf{2}$ & 12 & Flange/observation port \\
\hline $\mathbf{R} 3$ & 12 & Saltwell \\
\hline $\mathbf{R 4}$ & 4 & Breather filter, G1 housing \\
\hline R5 & 4 & Flange \\
\hline R6 & 12 & Flange \\
\hline R7 & 12 & Flange, dip tubes \\
\hline R8 & 4 & Liquid level reel (manual tape) \\
\hline $\mathbf{R} 9$ & 42 & Manhole, below grade \\
\hline Nonmin & 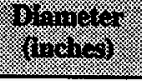 & 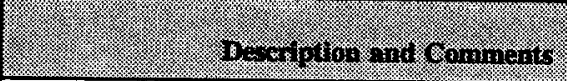 \\
\hline N1 & 3 & Line V-296 \\
\hline $\mathbf{N} 2$ & 3 & Line V-297 \\
\hline N3 & 3 & Spare \\
\hline N4 & 3 & Line to $\operatorname{tank}$ B-203 \\
\hline
\end{tabular}

Notes:

'Alstad (1993)
'Vitro Engineering Corporation (1986) 
Figure 2-2. Tank 241-B-204 Cross Section.

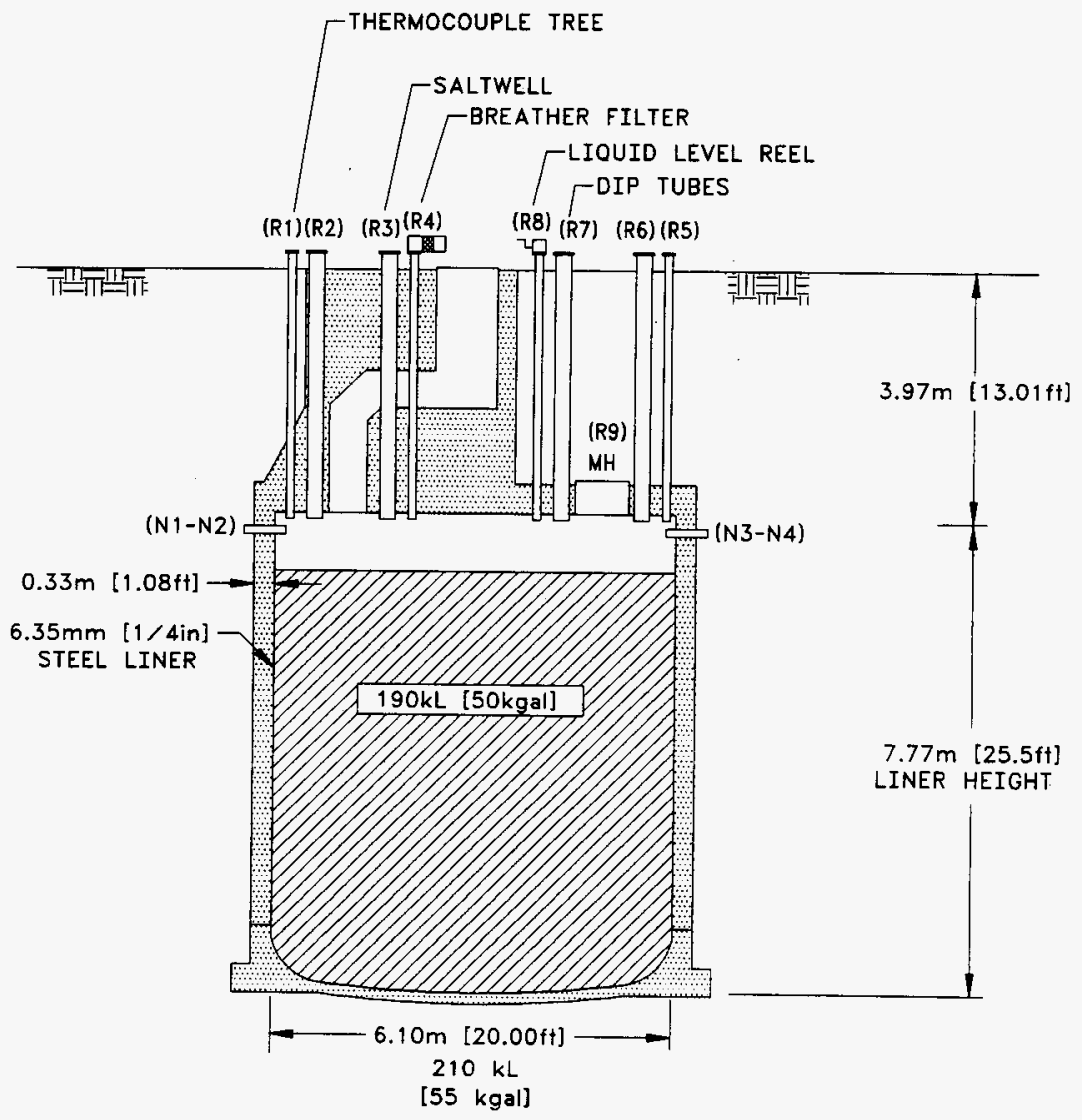




\subsection{PROCESS KNOWLEDGE}

The following sections describe the transfer history of waste in tank 241-B-204, the process wastes that were transferred, and an estimate of current tank contents based on transfer history.

\subsubsection{Waste Transfer History}

Table 2-3 shows the waste transfer history for tank 241-B-204. B Plant 224 waste from the lanthanum fluoride process was transferred to tank 241-B-204 during the second and third quarters of 1952 . In the fourth quarter of 1952 and the first quarter of 1953, tank 241-B-204 received B Plant flush waste. From the second quarter of 1952 to the first quarter of 1953, the overflow from tank 241-B-204 was cascaded to a crib (Brevick et al. 1994b). Approximately $185 \mathrm{~kL}$ (49 kgal) of waste was left in the tank after the final transfer in 1974. Tank 241-B-204 was removed from service in 1978.

Table 2-3. Summary of Tank 241-B-204 Waste Transfer History. ${ }^{1}$

\begin{tabular}{|c|c|c|c|c|}
\hline \multirow[b]{2}{*}{$4 \times 1342$} & \multirow[b]{2}{*}{ 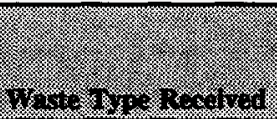 } & \multirow[b]{2}{*}{3110} & \multicolumn{2}{|c|}{ 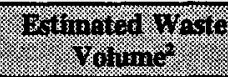 } \\
\hline & & & 46 & 8 \\
\hline $\begin{array}{c}224-U \\
\text { (B Plant) }\end{array}$ & 224 & 1952 & 943 & 249 \\
\hline B Plant & B Plant Flush & $1952-1953$ & 466 & 123 \\
\hline
\end{tabular}

Notes:

\footnotetext{
${ }^{1}$ Agnew (1995)

${ }^{2}$ Waste volumes and types are best estimates based on historical data.
}

\subsubsection{Historical Estimation of Tank Contents}

An estimate of the current contents of tank 241-B-204 based on historical transfer data is available from the Historical Tank Content Estimate for the Northeast Quadrant of the Hanford 200 East Area (Brevick et al. 1994a). The HTCE predictions have not been validated and should not be used as a basis for decisions affecting the waste in tank 241-B-204. Historical data used for the estimate include the Waste Status and Transaction Record Summary (WSTRS) for the Northeast Quadrant (Agnew et al. 1996), the Hanford Defined Waste: Chemical and Radionuclide Compositions (Agnew 1995), the Tank Layer Model (TLM) for the Northeast, Southwest, and Northwest Quadrants (Agnew et al. 1995), and the Hanford Tank Chemical and Radionuclide Inventories: HDW Model Rev. 3 (Agnew 1996). The Waste Status and Transaction Record Summary is a compilation of available waste transfer and volume status data. The Hanford Defined Waste 
(HDW) document provides the assumed typical compositions for Hanford waste types. In most cases, the available data are incomplete thereby reducing the reliability of the transfer data and the modeling results derived from it. The TLM takes the Waste Status and Transaction Record Summary data, models the waste deposition processes and, using additional data from the HDW document (which may introduce more error), generates an estimate of the tank contents. Therefore, these model predictions can only be considered an estimate that requires further evaluation using analytical data.

The TLM states that tank 241-B-204 contains $185 \mathrm{~kL}$ (49 kgal) of 224 waste from the $\mathrm{LaF}_{3}$ process (waste generated in the B-224 building) and $4 \mathrm{~kL} \mathrm{(1} \mathrm{kgal)} \mathrm{of} \mathrm{supernate} \mathrm{(see}$ Figure 2-3). The 224 sludge waste layer should contain the following: (1) very large quantities of iron, lanthanum, sodium, strontium, bismuth, fluoride, and nitrate, (2) a large quantity of carbonates, and (3) a trace amount of plutonium. The plutonium quantity may be enough to give this waste a slight activity. Table $2-4$ shows the waste constituents and their concentrations.

Figure 2-3. Tank Layer Model for Tank 241-B-204.

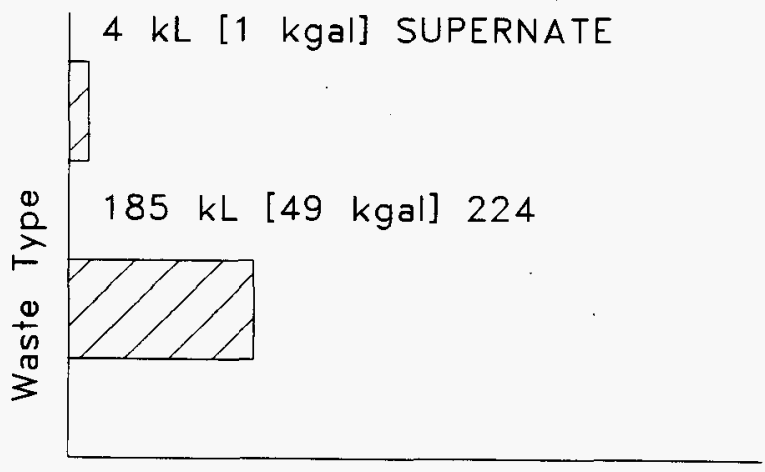

Waste Volume 
WHC-SD-WM-ER-581 Rev. 0

Table 2-4. Tank 241-B-204 Historical Inventory Estimate. ${ }^{1,2}$ (2 sheets)

\begin{tabular}{|c|c|c|c|}
\hline (1) & $100+000$ & 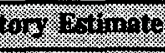 & \\
\hline 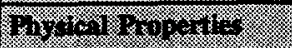 & s: & & \\
\hline Total solid waste & $2.55 \mathrm{E}+05 \mathrm{k}$ & kgal) & \\
\hline Heat load & O W (0 Btu/l & & \\
\hline Bulk density & $1.38 \mathrm{~g} / \mathrm{mL}$ & & \\
\hline Void fraction & 0.834 & & \\
\hline Water wt\% & 55.5 & & \\
\hline $\begin{array}{l}\text { Total organic carbon } \\
\text { (wt\% carbon wet) }\end{array}$ & 1.12 & & \\
\hline Chonisu Constinous & 1) & 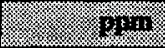 & 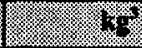 \\
\hline $\mathrm{Na}^{+}$ & 4.52 & 75,500 & 19,300 \\
\hline $\mathrm{Al}^{3+}$ & 0 & 0 & 0 \\
\hline $\mathrm{Fe}^{3+}$ (total Fe) & 0.361 & 14,600 & 3,740 \\
\hline $\mathrm{Cr}^{3+}$ & 0.00344 & 130 & 33.2 \\
\hline $\mathrm{Bi}^{3+}$ & 0.0604 & 9,170 & 2,340 \\
\hline $\mathrm{La}^{3+}$ & 0.237 & 23,900 & 6,100 \\
\hline $\mathrm{Ce}^{3+}$ & 0 & 0 & 0 \\
\hline $\mathrm{Zr}\left(\right.$ as $\left.\mathrm{ZrO}(\mathrm{OH})_{2}\right)$ & 0 & 0 & 0 \\
\hline $\mathrm{Pb}^{2+}$ & 0 & 0 & 0 \\
\hline $\mathrm{Ni}^{2+}$ & 0.00134 & 57.3 & 14.6 \\
\hline $\mathrm{Sr}^{2+}$ & 1.57 & 99,700 & 25,400 \\
\hline $\mathrm{Mn}^{4+}$ & 0.00386 & 154 & 39.3 \\
\hline $\mathrm{Ca}^{2+}$ & 0.244 & 7,100 & 1,810 \\
\hline$\overline{\mathrm{K}^{+}}$ & 0.228 & 6,460 & 1,650 \\
\hline $\mathrm{OH}^{-}$ & 4.92 & 60,700 & 15,500 \\
\hline $\mathrm{NO}_{3}^{-}$ & 1.33 & 59,800 & 15,300 \\
\hline $\mathrm{NO}_{2}^{-}$ & 0 & 0 & 0 \\
\hline $\mathrm{CO}_{3}^{2-}$ & 0.244 & 10,600 & 2,710 \\
\hline $\mathrm{PO}_{4}^{3-}$ & 0.0965 & 6,660 & 1,700 \\
\hline $\mathrm{SO}_{4}^{2-}$ & 0.00134 & 93.7 & 23.9 \\
\hline
\end{tabular}




\section{WHC-SD-WM-ER-581 Rev. 0}

Table 2-4. Tank 241-B-204 Historical Inventory Estimate. ${ }^{1,2}$ (2 sheets)

\begin{tabular}{|c|c|c|c|}
\hline 1) & 6) Congosite inren & 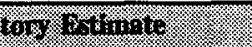 & 6 \\
\hline 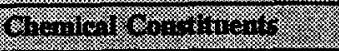 & 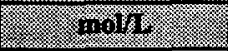 & (2) & 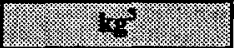 \\
\hline $\mathrm{Si}\left(\right.$ as $\left.\mathrm{SiO}_{3}^{2-}\right)$ & 0 & 0 & 0 \\
\hline $\mathrm{F}^{-}$ & 2.03 & 28,100 & 7,170 \\
\hline $\mathrm{Cl}^{-}$ & 0.0249 & 642 & 164 \\
\hline citrate & 0 & 0 & 0 \\
\hline EDTA $^{4}$ & 0 & 0 & 0 \\
\hline HEDTA $^{3-}$ & 0 & 0 & 0 \\
\hline $\mathrm{NTA}^{3-}$ & $\mathbf{0}$ & 0 & 0 \\
\hline glycolate & 0 & 0 & 0 \\
\hline acetate & 0 & 0 & 0 \\
\hline oxalate & 0.643 & 41,100 & 10,500 \\
\hline DBP & 0 & 0 & 0 \\
\hline NPH & 0 & 0 & 0 \\
\hline $\mathrm{CCl}_{4}$ & 0 & 0 & 0 \\
\hline hexone & 0 & 0 & 0 \\
\hline$(\mathrm{FeCN})_{6}^{4-}$ & 0 & 0 & 0 \\
\hline 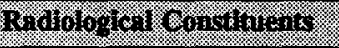 & .0101183 & 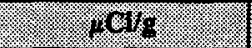 & 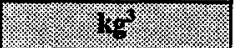 \\
\hline $\mathrm{Pu}$ & $\mathrm{n} / \mathbf{a}$ & 0.0100 & 0.0427 \\
\hline$\overline{\mathrm{U}}$ & 0 & 0 & 0 \\
\hline Cs & 0 & 0 & 0 \\
\hline $\mathrm{Sr}$ & 0 & 0 & 0 \\
\hline
\end{tabular}

Notes:

$\mathbf{n} / \mathbf{a}=$ not applicable

${ }^{1}$ Agnew (1996)

'The HTCE predictions have not been validated and should not be used as the basis for decisions affecting the waste in tank 241-B-204.

${ }^{3}$ Small differences appear to exist among the inventory values in this column and the inventories calculated from the two sets of concentrations. The differences are being evaluated. 


\subsection{SURVEILANCE DATA}

Tank 241-B-204 surveillance consists of surface level measurements (liquid and solid) and temperature monitoring (inside the tank waste and headspace). The data provide the basis for determining tank integrity. Liquid level measurements may indicate the presence of a major tank leak. Solid surface level measurements indicate physical changes and consistency of the solid layers. Tank 241-B-204 does not have liquid observation wells or drywells.

\subsubsection{Surface Level}

The surface level of the waste is monitored daily with a manual tape through riser 8 . The maximum allowed deviations from the $6.515 \mathrm{~m}(21.37 \mathrm{ft})$ baseline established for tank 241-B-204 are a $5.0 \mathrm{~cm}$ ( 2 in.) increase and a $5.0 \mathrm{~cm}$ (2 in.) decrease. Between 1991 and 1996, the surface level readings have ranged between $6.50 \mathrm{~m}(21.33 \mathrm{ft})$ and $6.51 \mathrm{~m}$ (21.36 ft). Figure 2-4 shows the tank volume history. The surface level on March 14, 1996 , was $6.5 \mathrm{~m}(21.33 \mathrm{ft})$.

\subsubsection{Internal Tank Temperatures}

Tank 241-B-204 has a single thermocouple tree with 12 thermocouples for monitoring temperatures. Documentation describing the thermocouple tree design and the elevations of the thermocouples was not available. No temperature data are available prior to 1975 , and there is a data gap between April 1982 and July 1989. Tank 241-B-204 temperatures currently are monitored semiannually. For plots of individual thermocouple readings, refer to Brevick et al. (1994b).

Between May 1975 and January 1996 , the mean temperature was $17^{\circ} \mathrm{C}\left(62.7^{\circ} \mathrm{F}\right)$ with a minimum of $7.2^{\circ} \mathrm{C}\left(45^{\circ} \mathrm{F}\right)$ and a maximum of $43^{\circ} \mathrm{C}\left(110^{\circ} \mathrm{F}\right)$. On January 9,1996 , the high temperature was $17^{\circ} \mathrm{C}\left(62.6^{\circ} \mathrm{F}\right)$ recorded by thermocouple 9 , and the low temperature was $14{ }^{\circ} \mathrm{C}\left(57 .{ }^{\circ} \mathrm{F}\right)$ recorded by thermocouple 12 . Figure $2-5$ shows a graph of high temperatures recorded between 1975 and 1996 . Brevick et al. (1994b) classify the maximum temperature of $43^{\circ} \mathrm{C}$, recorded July 5, 1989, as an outlier. All tank 241-B-204 thermocouples and most thermocouples for other 241-B 200-series tanks show a similar high reading on that same date. Considering the temperature history of the tank and the nature of the tank waste, it seems unlikely that tank waste temperatures were actually as high as $43{ }^{\circ} \mathrm{C}$. It is more likely that all the readings were subject to the same systematic error when they were collected. 


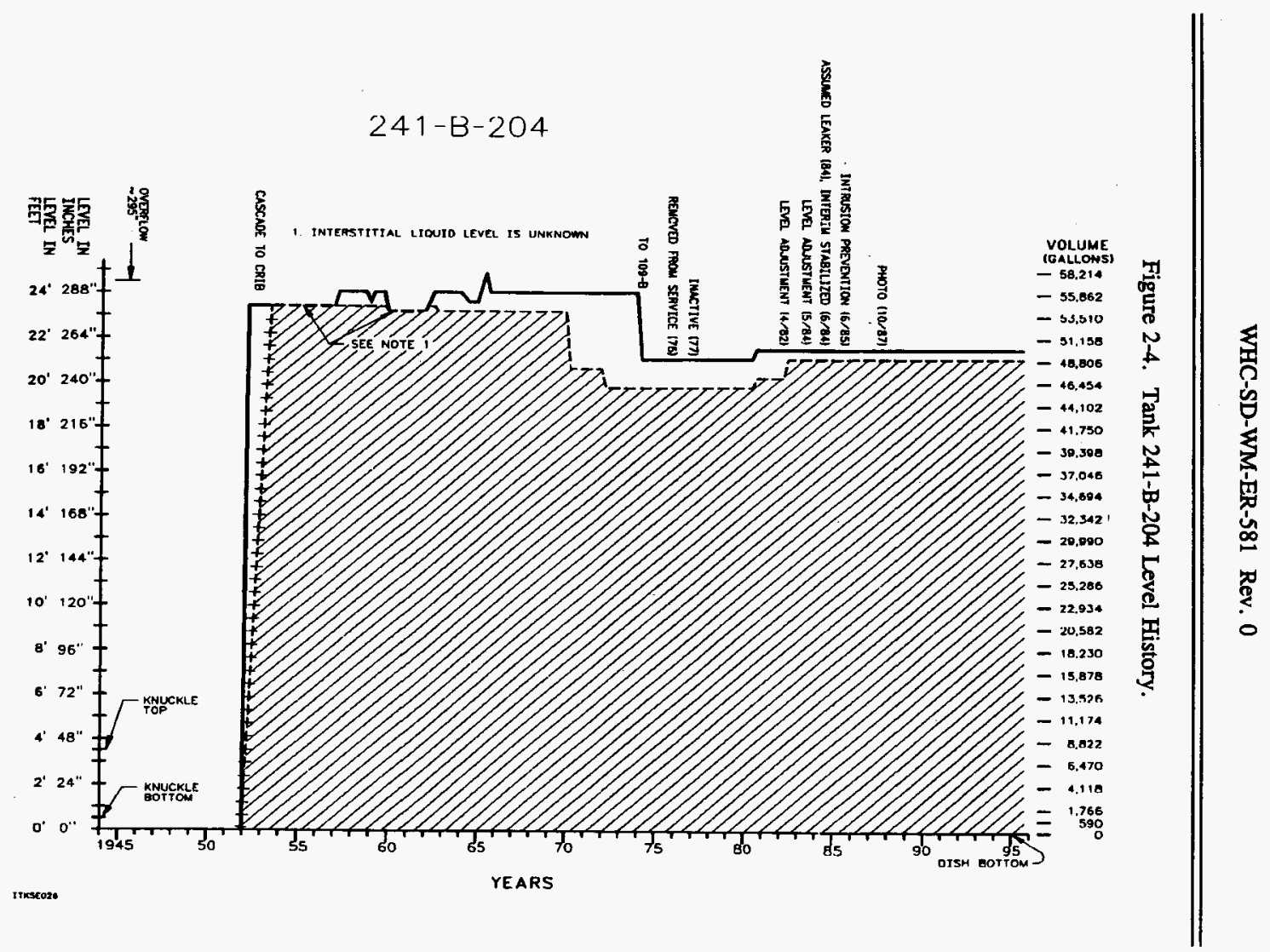


Figure 2-5. Tank 241-B-204 Weekly High Temperature Plot.

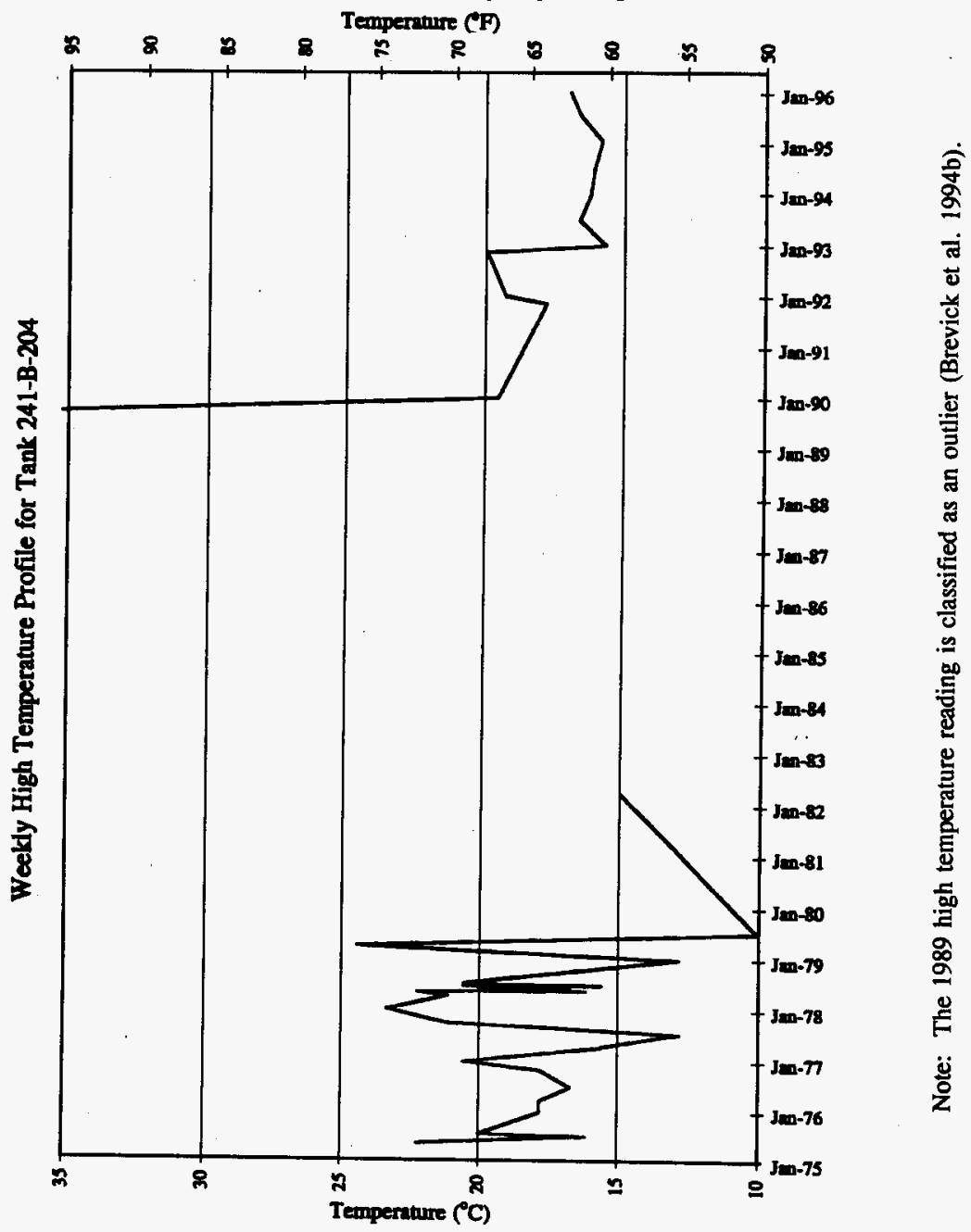




\subsubsection{Tank 241-B-204 Photograph}

Figure 2-6 is a montage of 1987 photographs of the tank 241-B-204 interior showing a thin supernate layer over a dark orange sludge. The in-tank photographs should correspond to the current tank contents since no transfers have taken place since the photographs were taken. The photographs show approximately $189 \mathrm{~kL}$ ( $50 \mathrm{kgal}$ ) of waste which equals a waste depth

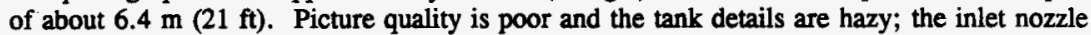
and temperature probe were overexposed because they were too close to the flash. 
WHC-SD-WM-ER-581 Rev. 0

This page intentionally left blank. 


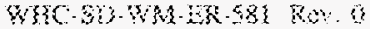

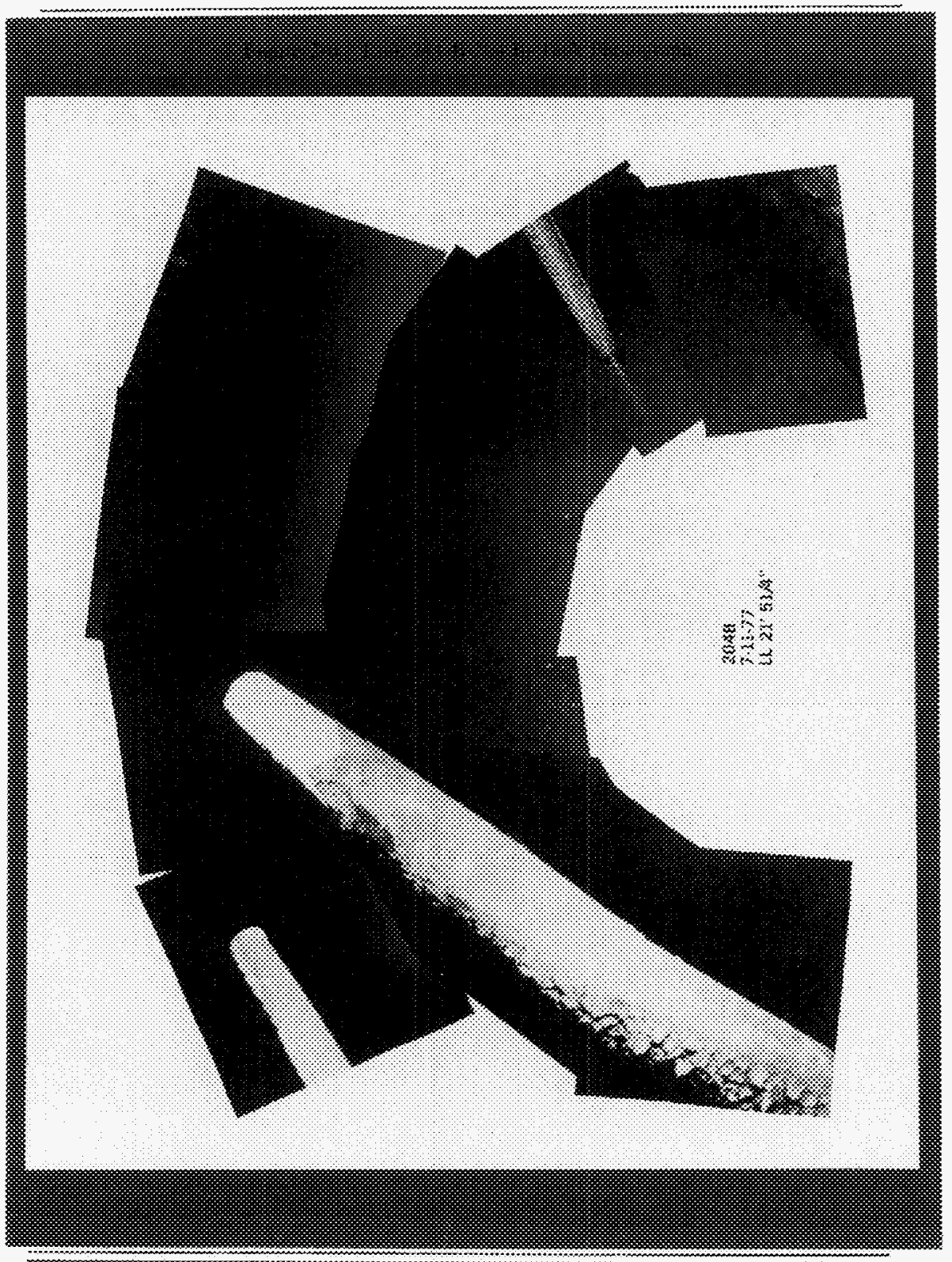


WHC-SD-WM-ER-581 Rev. 0

This page intentionally left blank. 


\subsection{TANK SAMPLING OVERVIEW}

This section describes the October 1995 sampling and analysis event for tank 241-B-204. Push-mode core samples were taken to satisfy the requirements of the safety screening DQO (Dukelow et al. 1995), and sampling and analysis were performed in accordance with the sampling and analysis plan (SAP) (Sasaki 1995). For further discussions of the sampling and analysis procedures, refer to the Tank Characterization Reference Guide (DeLorenzo et al. 1994).

\subsection{DESCRIPTION OF SAMPLING EVENT}

Two 14-segment push-mode core samples were collected from tank 241-B-204 between October 11 and October 19,1995. Cores 112 and 114 were taken from risers 2 and 7, respectively. Water was used as the HHF during sampling operations. A tracer (lithium bromide) was added to the HHF to gauge contamination of the segments by the HHF. A field blank and a sample of the HHF were also taken. Prior to core sampling, the tank headspace was sampled to determine the flammable gas concentration as outlined in the safety screening DQO. This sampling was done through riser 2 at approximately $1 \mathrm{~m}$ (3.3 ft) above the waste surface (or $4 \mathrm{~m} \mathrm{[13} \mathrm{ft]} \mathrm{below} \mathrm{the} \mathrm{top} \mathrm{of} \mathrm{the} \mathrm{riser)} \mathrm{(Sasaki} \mathrm{1996a).}$

All analyses were performed by the Westinghouse Hanford Company 222-S Laboratory in accordance with the SAP (Sasaki 1995). Table 3-1 summarizes the sampling and analysis requirements of the safety screening DQO (Dukelow et al. 1995). Table A-2 summarizes each core segment's riser number, sample depth, sample numbering, and drill string dose rate information.

Table 3-1. Integrated Data Quality Objective Requirements for Tank 241-B-204.

\begin{tabular}{|c|c|c|c|}
\hline $\begin{array}{l}\text { Sampling } \\
\text { Erent }\end{array}$ & 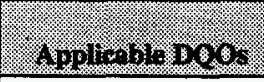 & Simpling Requirenents & $\begin{array}{l}\text { Inatitical } \\
\text { Requirements }\end{array}$ \\
\hline $\begin{array}{l}\text { Push-mode } \\
\text { core sampling }\end{array}$ & $\begin{array}{l}\text { Safety screening } \\
\text { (Dukelow et al. 1995) }\end{array}$ & $\begin{array}{l}\text { Core samples from a } \\
\text { minimum of two risers } \\
\text { separated radially to the } \\
\text { maximum extent possible }\end{array}$ & $\begin{array}{l}\text { Energetics } \\
\text { Moisture content } \\
\text { Total alpha activity } \\
\text { Density } \\
\text { Flammable gas } \\
\text { concentration }\end{array}$ \\
\hline
\end{tabular}




\subsection{SAMPLE HANDLING}

Cores 112 and 114 were received by the 222-S Laboratory between October 12 and October 20,1995, and were extruded between October 16 and October 26, 1995. The top two segments of core 112 and the top segment of core 114 contained drainable liquid. Small amounts of liner liquid were also recovered for most samples. All remaining segments of the two cores were full of smooth, soft, black sludge. No separable organic layer was observed in any segments. One field blank and one HHF sample were delivered to the 222-S Laboratory with core 114. Both cores were subsampled at the half-segment level for analysis. Table A-3 tabulates the subsampling scheme, the subsegment masses, and visual characteristics of the segments.

\subsection{SAMPLE ANALYSIS}

The safety screening DQO required analyses for thermal properties by DSC, moisture by TGA, fissile content by total alpha analysis, and bulk density. In addition to the core sample analyses, the tank headspace flammability was measured prior to core sampling.

Bromide analysis by IC and lithium analysis by ICP were required by the SAP to determine the amount of HHF contamination in the samples. During these measurements, results for other metals and anions were obtained and reported as requested by the SAP.

A GEA and ${ }^{90} \mathrm{Sr}$ analysis were performed on one solid subsample to provide radionuclide content information for the disposal of laboratory waste. The sample analyzed was from the lower half solids of segment 14 , core 112 , because this sample had the highest measured total alpha result.

All reported analyses were performed in accordance with approved laboratory procedures. Table A-4 lists the sample numbers and applicable analyses. Table A-5 displays analytical procedures by title and number. No deviations or modifications were noted by the laboratory. The appropriate quality control checks were run in conjunction with all chemical and physical analyses. Section 5.1.2 assesses the quality control results.

\subsection{DESCRIPTION OF HISTORICAL SAMPLING EVENT}

There was one historical sampling event reported for tank 241-B-204. The sample was analyzed in 1978 and referenced as sample number 1974 (Horton 1978). Because the sample was collected and analyzed prior to May 1989, the data do not meet current quality assurance requirements. Therefore, no decisions regarding the waste in tank 241-B-204 should be based upon these historical data. 
The specifics for this event such as the procedure, riser used, and depth at which the sample was taken are not available from historical records. The sample was received by the Chemical Analysis Laboratories at the 222-S Laboratory and was reported as black in color with a consistency of soft grease. Test results were reported on December 4, 1978. Both a water leach and an unspecified acid digestion method were performed on the sample. The results of the analyses revealed that the primary constituents by volume (percent) were sodium, nitrate, potassium, and carbonate. Appendix $\mathbf{D}$ shows the analytical results for this historical sample, and Section 5.2 compares the 1978 and 1995 analytical results. 
WHC-SD-WM-ER-581 Rev. 0

This page intentionally left blank. 


\subsection{ANALYTICAL RESULTS}

This section summarizes the analytical results of the October 1995 sampling of tank 241-B-204. The sampling and analysis were performed as directed in the SAP (Sasaki 1995). This plan integrated all documents related to sampling and analytical requirements including applicable DQOs. Analysis of the two cores were performed at the Westinghouse Hanford Company 222-S Laboratory.

Table 4-1 shows the locations of the analytical results.

Table 4-1. Analytical Data Presentation Tables.

\begin{tabular}{|l|l|}
\hline \multicolumn{1}{|c|}{ Unalosis } \\
\hline Chemical data summary & Table 4-2 \\
\hline Thermogravimetric analysis results & Appendix B, Table B-50 \\
\hline Differential scanning calorimetry & Appendix B, Table B-51 \\
\hline 1995 comprehensive analytical data & Appendix B \\
\hline HHF contamination check data & Appendix C \\
\hline
\end{tabular}

\subsection{DATA PRESENTATION}

This section summarizes the analytical results from the 1995 sampling of tank 241-B-204. The subsections below provide information about the chemical, physical, vapor, and HHF contamination check data. Data from the analysis of cores 112 and 114 were reported in 45-Day Safery Screening Results for Tank 241-B-204, Push Mode Cores 112 and 114 (Sasaki 1996a) and Final Report for Tank 241-B-204, Push Mode Cores 112 and 114 (Sasaki 1996b).

\subsubsection{Chemical Data Summary}

Table 4-2 shows the mean concentration estimates and inventories for the sludge and drainable liquid results separately and as a total tank inventory. Data from the two cores were combined to derive the overall concentration means for all analytes, with the exception of the radionuclides analyzed by GEA. The overall means reported are weighted means and were calculated by taking a simple mean of all subsegment values for a particular segment, averaging the segment means for an individual core to derive a core mean, and averaging the two core means to obtain an overall mean. When 50 percent or more of the individual 
Table 4-2. Chemical Data Summary for Tank 241-B-204. (3 sheets)

\begin{tabular}{|c|c|c|c|c|c|c|c|}
\hline r. & 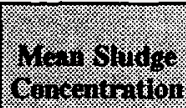 & 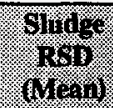 & ollower & 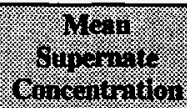 & 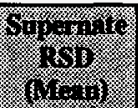 & 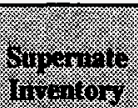 & min \\
\hline 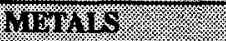 & m. & 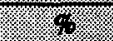 & ty & Siling & $\sqrt{*}$ & \% & ক্যে \\
\hline Aluminum & 65.3 & 36.5 & 14.4 & $<7.53$ & $\mathrm{n} / \mathrm{a}$ & $<0.0301$ & 14.4 \\
\hline Antimony & $<26.9$ & $\mathrm{n} / \mathrm{a}$ & $<5.92$ & $<9.08$ & $\mathrm{n} / \mathrm{a}$ & $<0.0363$ & $<5.96$ \\
\hline Arsenic & $<44.8$ & $\mathrm{n} / \mathrm{a}$ & $<9.86$ & $<15.1$ & $n / a$ & $<0.0604$ & $<9.92$ \\
\hline Barium & $<199$ & $\mathrm{n} / \mathrm{a}$ & $<43.8$ & $<7.53$ & $\mathrm{n} / \mathrm{a}$ & $<0.0301$ & $<43.8$ \\
\hline Beryllium & $<2.24$ & $\mathrm{n} / \mathrm{a}$ & $<0.493$ & $<0.753$ & $n / a$ & $<0.00300$ & $<0.496$ \\
\hline Bismuth & 48,400 & 5.8 & 10,700 & $<15.1$ & $\mathrm{n} / \mathrm{a}$ & $<0.0604$ & 10,700 \\
\hline Boron & 103 & 14.3 & 22.6 & $<7.53$ & $n / a$ & $<0.0301$ & 22.6 \\
\hline Cadmium & $<2.26$ & $\mathrm{n} / \mathrm{a}$ & $<0.497$ & $<0.753$ & $\mathrm{n} / \mathrm{a}$ & $<0.00301$ & $<0.500$ \\
\hline Calcium & 305 & 38.1 & 67.1 & $<15.1$ & $\mathrm{n} / \mathrm{a}$ & $<0.0604$ & 67.2 \\
\hline Cerium & 55.4 & 13.3 & 12.2 & $<15.1$ & $\mathrm{n} / \mathrm{a}$ & $<0.0604$ & 12.3 \\
\hline Chromium & 3,240 & 5.7 & 714 & 154 & 2.7 & 0.615 & 715 \\
\hline Cobalt & $<8.97$ & $\bar{n} / \mathbf{a}$ & $<1.98$ & $<3.02$ & $\mathrm{n} / \mathrm{a}$ & $<0.0121$ & $<1.99$ \\
\hline Copper & 22.1 & 80.4 & 4.87 & $<1.51$ & $\mathrm{n} / \mathrm{a}$ & $<0.00604$ & 4.88 \\
\hline Iron & 3,810 & 12.1 & 839 & $<7.53$ & $\mathrm{n} / \mathrm{a}$ & $<0.0301$ & 839 \\
\hline Lanthanum & 10,400 & 2.6 & 2,290 & $<7.53$ & $\mathrm{n} / \mathrm{a}$ & $<0.0301$ & 2,290 \\
\hline Lead & $<1,070$ & $\mathrm{n} / \mathrm{a}$ & $<236$ & $<15.1$ & $\mathrm{n} / \mathrm{a}$ & $<0.0604$ & $<236$ \\
\hline Magnesium & 82.8 & 20.2 & 18.2 & $<15.1$ & $\mathrm{n} / \mathrm{a}$ & $<0.0604$ & 18.3 \\
\hline Manganese & 14,800 & 2.7 & 3,270 & $<1.51$ & $\mathrm{n} / \mathrm{a}$ & $<0.00604$ & 3,270 \\
\hline Molybdenum & $<22.4$ & $\mathrm{n} / \mathrm{a}$ & $<4.93$ & $<7.53$ & $\mathrm{n} / \mathrm{a}$ & $<0.0301$ & $<4.96$ \\
\hline Neodymium & $<\overline{44.8}$ & $n / a$ & $<9.86$ & $<15.1$ & $\mathrm{n} / \mathrm{a}$ & $<0.0604$ & $<9.92$ \\
\hline
\end{tabular}




\begin{tabular}{|c|c|c|c|c|c|c|c|}
\hline G & 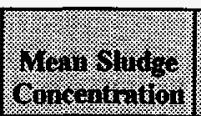 & 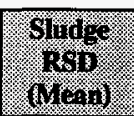 & (17\%) & 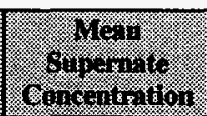 & 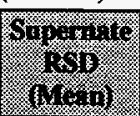 & 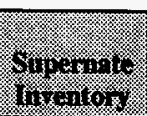 & Hiveritor \\
\hline 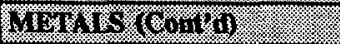 & t) & $\sqrt{1 / 28}$ & 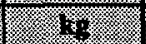 & I & স & (্) & (x) \\
\hline Nickel & 234 & 10.6 & 51.5 & $<3.02$ & $n / a$ & $<0.0121$ & 51.5 \\
\hline Phosphorous & 2,320 & 2.8 & 511 & 680 & 1.0 & 2.72 & 514 \\
\hline Potassium & 5,780 & 1.8 & 1,270 & 6,110 & 4.6 & 24.4 & 1,290 \\
\hline Samarium & $<44.8$ & $\mathrm{n} / \mathbf{a}$ & $<9.86$ & $<15.1$ & $\overline{n / a}$ & $<0.0604$ & $<9.92$ \\
\hline Selenium & $<44.9$ & $\mathrm{n} / \mathrm{a}$ & $<9.88$ & $<15.1$ & $n / a$ & $<0.0604$ & $<9.94$ \\
\hline Silicon & 1,070 & 22.8 & 236 & 60.9 & 10.0 & 0.244 & \begin{tabular}{|l|}
236 \\
\end{tabular} \\
\hline Silver & $<5.56$ & $\mathrm{n} / \mathrm{a}$ & $<1.22$ & $<1.51$ & $n / a$ & $<0.00604$ & $<1.23$ \\
\hline Sodium & 26,200 & 2.3 & 5,770 & 28,000 & 2.2 & 112 & 5,880 \\
\hline Strontium & 391 & 6.8 & 86.1 & $<1.51$ & $\mathrm{n} / \mathrm{a}$ & $<0.00604$ & 86.1 \\
\hline Sulfur & 95.0 & 8.4 & 20.9 & 134 & 3.7 & 0.536 & 21.4 \\
\hline Thallium & $<89.6$ & $n / a$ & $<19.7$ & $<30.2$ & $n / a$ & $<0.121$ & $<19.8$ \\
\hline Titanium & $<6.98$ & $\mathrm{n} / \mathrm{a}$ & $<1.54$ & $<1.51$ & $\mathrm{n} / \mathrm{a}$ & $<0.00604$ & $<1.55$ \\
\hline Uranium & $<240$ & $n / a$ & $<52.8$ & 164 & 7.9 & 0.656 & $<53.5$ \\
\hline Vanadium & $<22.4$ & $\mathrm{n} / \mathrm{a}$ & $<4.93$ & $<7.53$ & $n / a$ & $<0.0301$ & $<4.96$ \\
\hline Zinc & 53.7 & 39.7 & 11.8 & 2.62 & 41.2 & 0.0105 & 11.8 \\
\hline Zirconium & $<4.52$ & $\mathrm{n} / \mathrm{a}$ & $<0.995$ & $<1.51$ & $\pi / \mathbf{a}$ & $<0.00604$ & $<1.00$ \\
\hline WMors & . $4 \mathrm{gg}$ & . & 4 & (1) & (3) & (: & 80 \\
\hline Chloride & 693 & 7.3 & 153 & 592 & 8.8 & 2.37 & 155 \\
\hline Fluoride (soluble) & 7,080 & 9.5 & 1,560 & 6,240 & 6.0 & 25.0 & 1,580 \\
\hline Nitrate & 52,900 & 7.4 & 11,600 & 45,900 & 3.8 & 184 & 11,800 \\
\hline Nitrite & 694 & 18.5 & 153 & 1,730 & 14.2 & 6.92 & 160 \\
\hline
\end{tabular}


Table 4-2. Chemical Data Summary for Tank 241-B-204. (3 sheets)

\begin{tabular}{|c|c|c|c|c|c|c|c|}
\hline 政: & $\begin{array}{l}\text { Wein Shitg } \\
\text { Goncenorition }\end{array}$ & 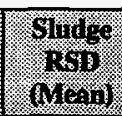 & $\begin{array}{l}\text { Sindyo } \\
\text { Inreatery }\end{array}$ & 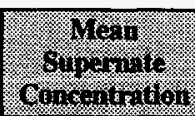 & 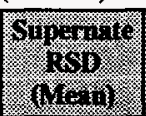 & 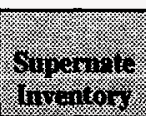 & 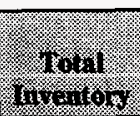 \\
\hline 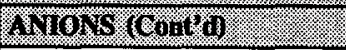 & (3) & 13 & 18. & (1. & 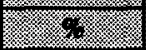 & 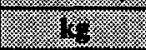 & (3. \\
\hline Oxalate & 1,710 & 5.5 & 376 & $<356$ & $n / a$ & $<1.42$ & 376 \\
\hline Phosphate (soluble) & 3,620 & 8.8 & 797 & 2,060 & 12.4 & 8.24 & 805 \\
\hline Sulfate & 648 & 12.0 & 143 & 785 & 34.2 & 3.14 & 146 \\
\hline 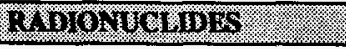 & igro & (3) & (4) & $16 \mathrm{~cm}$ & (4) & (4) & 0 \\
\hline${ }^{241} \mathrm{Am}^{1}$ & $<0.0833$ & $n / a$ & $<18.3$ & $n / a$ & $\mathrm{n} / \mathrm{a}$ & $n / a$ & $<18.3$ \\
\hline${ }^{60} \mathrm{Co}^{1}$ & $<0.0131$ & $\mathbf{n} / \mathbf{a}$ & $<2.88$ & $\mathrm{n} / \mathrm{a}$ & $\mathrm{n} / \mathrm{a}$ & $n / a$ & $<2.88$ \\
\hline${ }^{137} \mathrm{Cs}^{1}$ & $<0.0314$ & $\mathrm{n} / \mathrm{a}$ & $<6.91$ & $n / a$ & $n / a$ & $n / a$ & $<6.91$ \\
\hline${ }^{154} \mathrm{Eu}^{1}$ & $<0.0357$ & $n / a$ & $<7.86$ & $\mathrm{n} / \mathrm{a}$ & $n / a$ & $n / a$ & $<7.86$ \\
\hline${ }^{155} \mathrm{Eu}^{1}$ & $<0.0380$ & $\mathrm{n} / \mathrm{a}$ & $<8.37$ & $\mathrm{n} / \mathbf{a}$ & $n / a$ & $n / a$ & $<8.37$ \\
\hline${ }^{90} \mathrm{Sr}^{1}$ & $5.00 \mathrm{E}-03$ & $24.0^{2}$ & 1.10 & $\mathrm{n} / \mathbf{a}$ & $\mathrm{n} / \mathrm{a}$ & $\mathrm{n} / \mathbf{a}$ & 1.10 \\
\hline Total alpha & 0.264 & 7.2 & 58.1 & $9.98 \mathrm{E}-05$ & 8.2 & 3.99E-04 & 58.1 \\
\hline 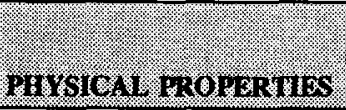 & sugen Men & 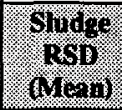 & 18 & 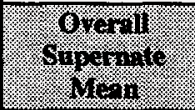 & 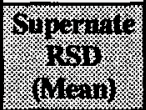 & 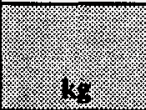 & 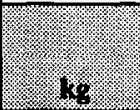 \\
\hline Weight-percent water ${ }^{3}$ & $77.1 \%$ & 0.9 & 170,000 & $89.1 \%$ & 0.2 & 3,540 & 173,000 \\
\hline Density & $1.19 \mathrm{~g} / \mathrm{mL}$ & 2.0 & 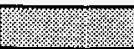 & $1.05 \mathrm{~g} / \mathrm{mL}$ & 0.5 & (5) & (1) \\
\hline
\end{tabular}

Notes:

$\mathrm{n} / \mathbf{a}=$ not applicable

${ }^{1}$ Radionuclide means are based on the results from one sample/duplicate pair taken from the lower half solids of segment 14 , core 112 .

${ }^{2}$ Relative standard deviation of the data is shown because only one sample/duplicate pair was analyzed.

${ }^{3}$ Weight-percent water overall means are based on results corrected for HHF contamination. 
sample/duplicate measurements had detected results, the overall mean was reported as a detected value. Conversely, when results for more than 50 percent of the individual sample/duplicate results were nondetected, the overall mean was reported as a less than $(<)$ value.

The GEA and ${ }^{90} \mathrm{Sr}$ were performed only on the lower half solids of segment 14, core 112 . Consequently, the overall means for ${ }^{241} \mathrm{Am},{ }^{60} \mathrm{Co},{ }^{137} \mathrm{Cs},{ }^{154} \mathrm{Eu},{ }^{155} \mathrm{Eu}$, and ${ }^{90} \mathrm{Sr}$ are based on the results from one sample/duplicate pair and may not represent the entire tank contents.

Table 4-2 shows the overall means in columns 2 and 5 for the sludge and supernate portions of the waste, respectively. Appendix B lists the original subsegment analytical data.

Relative standard deviations of the mean (RSD [Mean]), defined as 100 times the standard deviation of the mean divided by the tank mean. The standard deviation was computed using a nested analysis of variance model and restricted maximum likelihood methods. They are reported in columns 3 and 6 and were calculated only for analytes which had 50 percent or more of their individual sample/duplicate results above the detection limit.

The sludge inventory in column 4 was calculated by multiplying the overall mean by the sludge density $(1.19 \mathrm{~g} / \mathrm{mL})$ and the sludge waste volume $(185,000 \mathrm{~L}$ [49,000 gal]), then dividing by a unit conversion factor of $1 \mathrm{E}+06$. The supernate inventory in column 7 was calculated by multiplying the overall mean by the supernate waste volume $(4,000 \mathrm{~L}$ [1,000 gal]) and dividing by a unit conversion factor of $1 E+06$. Total inventory results in column 8 are the sum of the sludge and supernate inventories. Using the respective estimates for the sludge and supernate volumes and densities, the total sludge mass is estimated to be $220,000 \mathrm{~kg}$, and the total supernate mass is estimated to be $3,970 \mathrm{~kg}$.

\subsubsection{Physical Data Summary}

Thermal analyses were performed on tank 241-B-204 core samples to satisfy the requirements of the safety screening DQO (Dukelow et al. 1995) which dictated that TGA and DSC be performed on both solid and liquid phases of the waste samples. Density determinations were also performed.

4.1.2.1 Thermogravimetric Analysis. In a TGA, the mass of a sample is measured while its temperature is increased at a constant rate. A gas, such as nitrogen or air, is passed over the sample during the heating to remove any released gases. A decrease in the weight of a sample represents a loss of gaseous matter from the sample through evaporation or through a reaction that forms gas phase products. 
Weight-percent water by TGA was performed by the 222-S Laboratory under a nitrogen purge using procedures LA-560-112, Rev. B-1 (Mettler ${ }^{1}$ ) and LA-514-114, Rev. C-1 (Perkin-Elmer ${ }^{2}$.

Table B-50 shows the TGA percent water data for tank 241-B-204. The HHF-corrected mean weight-percent water result for drainable liquid samples was 89.1 percent with an RSD of 0.2 percent. The HHF-corrected mean result for the sludge samples was 77.1 percent with an RSD of 0.9 percent. All weight-percent water sample means were calculated by equal weighting of the two cores, the segments, and the subsegments using values corrected for HHF contamination based on the bromide results. Sample S95T003260 was rerun because of an instrument error, and a triplicate was analyzed on sample S95T003278 because of the large discrepancy between the original and duplicate results. The laboratory did not encounter other problems.

4.1.2.2 Differential Scanning Calorimetry. In a DSC analysis, heat absorbed or emitted by a substance is measured while the substance is exposed to a linear increase in temperature. As the substance is being heated, a gas such as nitrogen is passed over the waste material to remove any gases being released. The onset temperature for an endothermic event (characterized by or causing the absorption of heat) or an exothermic event (characterized by or causing the release of heat) is determined graphically.

The DSC analyses were performed by the 222-S Laboratory under a nitrogen atmosphere using procedures LA-514-113, Rev. C-1 (Mettler ${ }^{\text {TM}}$ ) or LA-514-114, Rev. C-1 (Perkin-Elmer ${ }^{\mathrm{TM}}$ ).

No exothermic reactions were observed, and the only endothermic transition exhibited was that associated with the evaporation of free and interstitial water. Because there were no exothermic reactions, the calculation of a 95 percent confidence interval as specified by the safety screening DQO (Dukelow et al. 1995) was not necessary. Table B-51 shows the DSC results and includes, for each sample, the temperature range and enthalpy for the single endothermic transition.

4.1.2.3 Density. Bulk density measurements were performed on solid samples using procedure LO-160-103, Rev. A-7. Specific gravity measurements were performed on the liquid samples using procedure LA-510-112, Rev. C-3. The mean density of the sludge was $1.19 \mathrm{~g} / \mathrm{mL}$, and that of the drainable liquid was $1.05 \mathrm{~g} / \mathrm{mL}$. Table B-52 shows the analytical data.

\footnotetext{
'Mettler is a registered trademark of Mettler Electronics, Anaheim, CA.

${ }^{2}$ Perkin-Elmer is a registered trademark of Perkins Research and Manufacturing Company, Inc., Canoga, Park CA.
} 


\subsubsection{Tank Headspace Flammability}

The sampling of tank 241-B-204 headspace was performed prior to core sampling. The tank vapors were field tested using a combustible gas meter; the flammable vapor content was determined to be 0 percent of the lower flammability limit, indicating no flammability concerns. In addition, the concentrations of oxygen (20.7 percent), total organic vapors (1.4 ppm), and ammonia (2 ppm) were measured.

\subsubsection{Hydrostatic Head Fluid Contamination Check}

Hydrostatic head fluid was used in conjunction with the 1995 core samplings, and lithium bromide was used as a tracer in the HHF to estimate the degree of sample contamination. This check, through chemical analyses for lithium and bromide, was prescribed by the SAP (Sasaki 1995). The analytical results indicated that both elements were present in detectable quantities in all sludge subsegments and most drainable liquid samples (see Appendix C). Based on the bromide results, the HHF contamination was less than 2 percent for all samples. The HHF correction factors were applied to the weight-percent water analytical data for all subsegments; the HHF correction factors were not applied to any other analyte. The bromide values were chosen for the HHF correction factors because bromide is less likely than lithium to form insoluble compounds with tank waste. When lithium and bromide results were compared, the equivalents of lithium recovered were less than those for bromide in general. An overall mean and tank inventory were not calculated for these two analytes because they are not constituents of the tank waste. 
WHC-SD-WM-ER-581 Rev. 0

This page intentionally left blank. 


\subsection{INTERPRETATION OF CHARACTERIZATION RESULTS}

This section discusses the overall quality and consistency of the current sampling results for tank 241-B-204 and assesses and compares these results to historical information and program requirements.

\subsection{ASSESSMENT OF SAMPLING AND ANALYTICAL RESULTS}

This section evaluates sampling and analysis factors that may impact data interpretation. These factors are used to assess the overall data quality and consistency and to identify data limitations.

\subsubsection{Field Observations}

The safety screening DQO (Dukelow et al. 1995) requirement to sample from at least two widely-spaced risers was fulfilled. Therefore, a horizontal and vertical comparison of the analytical results was possible and gave an estimate of waste constituent distribution. No sampling problems were noted. All samples achieved 100 percent recovery.

\subsubsection{Quality Control Assessment}

The usual quality control assessment includes evaluating the appropriate standard recoveries, spike recoveries, duplicate analyses, and blanks that are performed in conjunction with the chemical analyses. All pertinent quality control tests were conducted on the 1995 core samples, enabling a full assessment of data accuracy and precision. The SAP (Sasaki 1995) established the specific criteria for the primary analytes (TGA, DSC, bulk density, total alpha activity, lithium, and bromide), and the remainder of the analytes were governed by the laboratory criteria (DOE 1995). Sample and duplicate pairs that had one or more quality control results outside the specified criteria are identified by footnoting the data in Appendices $\mathrm{B}$ and $\mathrm{C}$.

The standard and spike recovery results provide an estimate of analysis accuracy. If a standard or spike recovery is above or below the given criterion, the analytical results may be biased high or low, respectively. The precision is estimated by the relative percent difference (RPD), which is defined as the absolute value of the difference between the primary and duplicate samples, divided by their mean, times one hundred. The lithium analyses had two low spike recoveries, a result of the lithium concentration in the sample being two orders of magnitude greater than the spike. When the sample concentration is more than four times larger than the spike, the error introduced in calculating the spike recovery becomes large and results in a poor recovery. The lithium analyses also had three high RPDs. Several spike recoveries and RPDs were outside the target level for total alpha 
activity. This was caused by low sample activities and high dissolved solids in the samples and by possible self-shielding. Several standard recoveries, conducted with total alpha activity, were outside the SAP criterion; TGA had three RPDs, and bulk density had one RPD outside the limits. Finally, no sample exceeded the criterion for preparation blanks; therefore, contamination was not a problem.

In summary, almost all quality control results for the primary analytes listed above were within the boundaries specified in the SAP. The few discrepancies noted should not impact data validity or use. None of the remaining data in this report had quality control violations (ICP, IC, and GEA analytes).

\subsubsection{Data Consistency Checks}

Comparing different analytical methods can help to assess data consistency and quality. The quantity of data available enabled calculations of mass and charge balances and comparisons of the ICP phosphorus and sulfur results with the IC phosphate and sulfate results, respectively.

5.1.3.1 Comparison of Results from Different Analytical Methods. The following data consistency check compares phosphate and sulfate results from two analytical methods. A close correlation between the two methods strengthens the credibility of both results, whereas a poor correlation may bring the data reliability into question. Table 4-2 shows the analytical mean results.

For supernate, the ICP mean total phosphorous result was $680 \mu \mathrm{g} / \mathrm{mL}$ (equivalent to $2,090 \mu \mathrm{g} / \mathrm{mL}$ phosphate). The IC mean phosphate result was $2,060 \mu \mathrm{g} / \mathrm{mL}$. The ICP and IC results agree with an RPD of only 1.4 percent.

For sludge, the analytical phosphorous mean result as determined by ICP was $2,320 \mu \mathrm{g} / \mathrm{g}$ (equivalent to $7,110 \mu \mathrm{g} / \mathrm{g}$ phosphate). The IC phosphate mean result was $3,620 \mu \mathrm{g} / \mathrm{g}$, which yielded an RPD between the two phosphate results of 65 percent. The low IC phosphate value is caused by the inability of the water-leach sample-preparation procedure to solubilize the water-insoluble phosphate compounds prior to IC analysis. The acid-digestion procedure, which is used to dissolve the sludge prior to ICP analysis, is much more likely to yield soluble phosphate that is detected as total phosphorous. Comparing IC and ICP results for phosphate suggests that only 51 percent of the phosphate in the sludge is water soluble.

The mean total sulfur value for the supernate, as determined by ICP, was $134 \mu \mathrm{g} / \mathrm{mL}$ (equivalent to $401 \mu \mathrm{g} / \mathrm{mL}$ sulfate). The mean sulfate value, as determined by IC, was $785 \mu \mathrm{g} / \mathrm{mL}$; the RPD for the two values is 64.8 percent. The poor agreement between the two sulfate values may be caused in part by the IC supernate samples being diluted to very near the method's detection limit for sulfate. Therefore, the poor agreement between the ICP and IC supernate sulfate values may be caused by inaccuracies associated with quantifying sulfate near the IC detection limit. In contrast, the ICP sulfur values tended to 
fall between five and 10 times the ICP detection limit for sulfur. Therefore, the sulfate value calculated from the ICP sulfur results may be a better estimate of the sulfate concentration in the supernate than the IC sulfate value.

The mean ICP sulfur value for the sludge of $95.0 \mu \mathrm{g} / \mathrm{g}$ converts to $284 \mu \mathrm{g} / \mathrm{g}$ of sulfate. The IC sulfate mean result was $648 \mu \mathrm{g} / \mathrm{g}$, which yielded an RPD of 78 percent between the two sulfate results. Again, the IC sulfate values for the diluted, water-digested samples were near the detection limit of sulfate and the ICP sulfur values tended to be between five and 10 times the ICP detection limit for sulfur. Furthermore, the water leach of the sludge may not have removed all sulfate from the sludge. Therefore, the sulfate value calculated from the ICP sulfur results may be a better estimate of the sulfate concentration in the sludge than the IC sulfate value.

5.1.3.2 Mass and Charge Balances. The principle objective in performing mass and charge balances is to determine whether the measurements are self-consistent. When calculating the balances, only the analytes detected at a concentration of $3,000 \mu \mathrm{g} / \mathrm{g}$ or greater were considered (see Table 4-2). Only the sludge portion of the waste was considered because it comprises 98 percent of the tank contents.

Except for sodium and potassium, all cations were assumed present in their most common hydroxide or oxide form, and the concentrations of the assumed species were calculated stoichiometrically (see Table 5-1). Because precipitates are neutral species, all positive charge was attributed to the sodium and potassium cations. The anionic analytes were assumed present as sodium/potassium salts and were expected to balance the positive charge exhibited by the cations (see Table 5-2). Phosphorus is assumed present as the soluble phosphate ion and the insoluble compound bismuth phosphate. The concentration of the insoluble phosphate was found by subtracting soluble phosphate, as determined by IC, from the total amount of phosphate calculated from total phosphorous by ICP. The concentrations of the cationic species, the anionic species, and the percent water were used to calculate the mass balance. The uncertainty estimates (RSDs) associated with each analyte are also given in the tables. Uncertainty estimates for the cation and anion totals and the overall uncertainty (see Table 5-3), were computed by a statistical technique known as the propagation of errors (Nuclear Regulatory Commission 1988).

The mass balance was calculated from the formula below. The factor 0.0001 is the conversion factor from $\mu \mathrm{g} / \mathrm{g}$ to weight percent.

$$
\begin{aligned}
\text { Mass balance } & =\% \text { Water }+0.0001 \times\{\text { Total Analyte Concentration }\} \\
& =\% \text { Water }+0.0001 \times\left\{\mathrm{BiPO}_{4}+\mathrm{Bi}_{2} \mathrm{O}_{3}+\mathrm{Cr}(\mathrm{OH})_{3}+\mathrm{FeO}(\mathrm{OH})+\mathrm{La}(\mathrm{OH})_{3}\right. \\
& \left.+\mathrm{MnO}(\mathrm{OH})+\mathrm{Na}^{+}+\mathrm{K}^{+}+\mathrm{F}^{-}+\mathrm{NO}_{3}^{-}+\mathrm{PO}_{4}^{-3}\right\}
\end{aligned}
$$

The total analyte concentrations calculated from the above equation is $203,000 \mu \mathrm{g} / \mathrm{g}$. The sludge mean weight percent water obtained from TGA (see Table 4-2) is 77.1 percent or $771,000 \mu \mathrm{g} / \mathrm{g}$. The mass balance resulting from adding the percent water to the total analyte concentration is 97.4 percent (see Table 5-3). 
WHC-SD-WM-ER-581 Rev. 0

Table 5-1. Cation Mass and Charge Data.

\begin{tabular}{|c|c|c|c|c|c|}
\hline \multirow[b]{2}{*}{101818} & 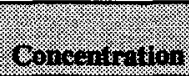 & \multirow{2}{*}{40} & 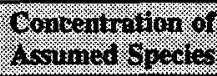 & 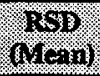 & o \\
\hline & 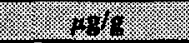 & & 2,38 & 8 & rects \\
\hline Bismuth & 7,680 & $\mathrm{BiPO}_{4}$ & 11,200 & 5.8 & 0 \\
\hline Bismuth & 40,700 & $\mathrm{Bi}_{2} \mathrm{O}_{3}$ & 45,400 & 5.8 & 0 \\
\hline Chromium & 3,240 & $\mathrm{Cr}(\mathrm{OH})_{3}$ & 6,420 & 5.7 & 0 \\
\hline Iron & 3,810 & $\mathrm{FeO}(\mathrm{OH})$ & 6,060 & 12.1 & 0 \\
\hline Lanthanum & 10,400 & $\mathrm{La}(\mathrm{OH})_{3}$ & 14,200 & 2.6 & 0 \\
\hline Manganese & 14,800 & $\mathrm{MnO}(\mathrm{OH})$ & 23,700 & 2.7 & 0 \\
\hline Potassium & 5,780 & $\mathrm{~K}^{+}$ & 5,780 & 1.8 & 148 \\
\hline Sodium & 26,200 & $\mathrm{Na}^{+}$ & 26,200 & 2.3 & 1,140 \\
\hline Total & & & 139,000 & 2.1 & 1,290 \\
\hline
\end{tabular}

Table 5-2. Anion Mass and Charge Data.

\begin{tabular}{|c|c|c|c|c|c|}
\hline \multirow[b]{2}{*}{1.121018} & $0,110-11,941 \%$ & \multirow{2}{*}{$4 x_{0+210}$} & 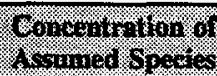 & 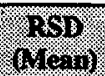 & $0101 \%$ \\
\hline & $\sqrt{150}$ & & 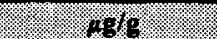 & 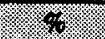 & 100 \\
\hline Fluoride & 7,080 & $F$ & 7,080 & 9.5 & 373 \\
\hline Nitrate & 52,900 & $\mathrm{NO}_{3}^{-}$ & 52,900 & 7.4 & 853 \\
\hline Phosphate & 3,620 & $\mathrm{PO}_{4}{ }^{3-}$ & 3,620 & 8.8 & 114 \\
\hline Total & & & 63,600 & 6.3 & 1,340 \\
\hline
\end{tabular}

Table 5-3. Mass Balance Totals.

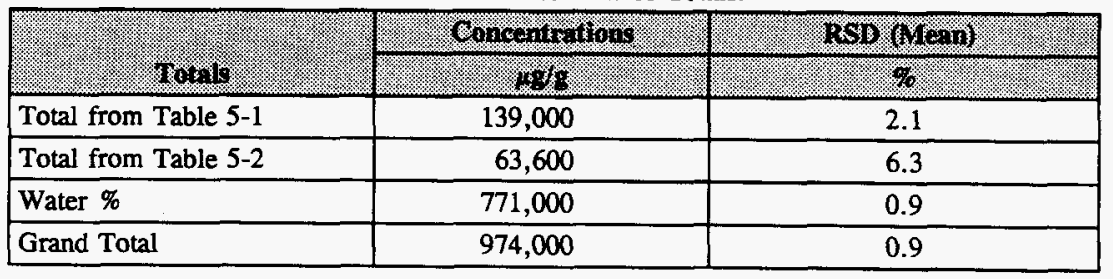


The following equations demonstrate the derivation of total cations and total anions; the charge balance is the ratio of these two values.

Total cations $(\mu \mathrm{eq} / \mathrm{g})=\left[\mathrm{Na}^{+}\right] / 23.0+\left[\mathrm{K}^{+}\right] / 39.1=1,290 \mu \mathrm{eq} / \mathrm{g}$

Total anions $(\mu \mathrm{eq} / \mathrm{g})=\left[\mathrm{F}^{-}\right] / 19.0+\left[\mathrm{NO}_{3}^{-}\right] / 62.0+\left[\mathrm{PO}_{4}^{3-}\right] / 31.7=1,340 \mu \mathrm{eq} / \mathrm{g}$

The charge balance obtained by dividing the sum of the positive charge by the sum of the negative charge was 0.96 .

In summary, the above calculations yield reasonable mass and charge balance values (close to 1.00 for charge balance and 100 percent for mass balance), indicating that the analytical results and the assumptions used in interpreting them are generally self-consistent.

\subsection{COMPARISON OF HISTORICAL WITH ANALYTICAL RESULTS}

Based on the tank waste transfer history, tank contents have not changed since 1974 . Therefore, it is possible to compare historical sludge sampling from 1978 and the sludge results from the 1995 core sampling event. The 1995 sampling was well documented and should be representative of the tank contents. No specific information was available about sample location, depth, or number of samples in the 1978 data so it is not known how representative these samples were. These data are not validated and are for comparison only. No decisions regarding the waste in tank 241-B-204 should be based on the 1978 data.

Table 5-4 compares the two data sets. The 1978 results were derived by summing the water soluble and acid (fusion) results for each analyte (see Appendix D). Only one plutonium value was available from the 1978 results. It is unknown whether this represents a sum of all plutonium isotopes or just one of them. When comparing to the 1995 total alpha activity result, it was assumed that plutonium was the only alpha emitter present, and that all plutonium was present as ${ }^{239} \mathrm{Pu}$. The conversion factor of $0.0615 \mathrm{Ci} / \mathrm{g}$ was used to convert the 1978 plutonium result of $9.74 \mathrm{E}-06 \mathrm{~g} / \mathrm{g}$ to $0.599 \mu \mathrm{Ci} / \mathrm{g}$ total alpha.

As seen from the large RPDs, most analytes do not compare well. The percent water and density results compare very well indicating the liquid content of the tank has remained unchanged. Although the waste-water content might have been expected to decrease between the two sampling events because of 17 years evaporation, very little evaporation may have occurred because the thermal driver for evaporation is very small, and the tank is passively ventilated. The large differences between the results of the two sampling events may be caused by different sampling depths and locations and the possibility that 1978 results do not represent the tank waste and may not be valid. 
Table 5-4. Comparisons of Sludge Data from the 1978 and 1995 Sampling Events for Tank 241-B-204.

\begin{tabular}{|c|c|c|c|}
\hline (- & 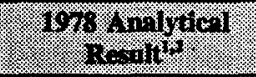 & 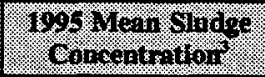 & 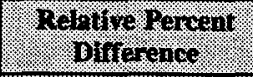 \\
\hline WHWMU & 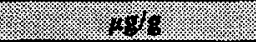 & 1606 & W. \\
\hline Aluminum & 88 & 65.3 & 29.6 \\
\hline Bismuth & 62,000 & 48,400 & 24.6 \\
\hline Chromium & 400 & 3,240 & 156 \\
\hline Iron & 29,000 & 3,810 & 154 \\
\hline Lanthanum & 20,100 & 10,400 & 63.6 \\
\hline Manganese & 1 & 14,800 & 200 \\
\hline Nickel & 300 & 234 & 24.7 \\
\hline Potassium & 4,000 & 5,780 & 36.4 \\
\hline Silicon & 1,420 & 1,070 & 28,1 \\
\hline Sodium & 14,000 & 26,200 & 60.7 \\
\hline ANOAS & 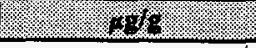 & 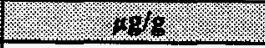 & 9. \\
\hline Chloride & 700 & 693 & 1.0 \\
\hline Fluoride & 2,000 & 7,080 & 112 \\
\hline Nitrate & 28,700 & 52,900 & 59.3 \\
\hline Nitrite & 3,000 & 694 & 125 \\
\hline Phosphate & 13,000 & 3,620 & 113 \\
\hline 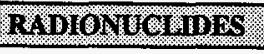 & acts & actg & - \\
\hline Total alpha & $0.599^{4}$ & 0.264 & 77.6 \\
\hline \multicolumn{3}{|c|}{ 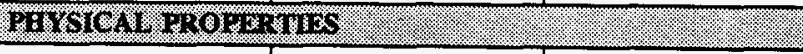 } & 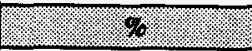 \\
\hline Bulk density & $1.14 \mathrm{~g} / \mathrm{mL}$ & $1.19 \mathrm{~g} / \mathrm{mL}$ & 4.3 \\
\hline Percent water & $76.0 \%$ & $77.1 \%$ & 1.4 \\
\hline
\end{tabular}

Notes:

'Brevick et al. (1994a)

These data are not validated and are for comparison only.

${ }^{3}$ Sasaki (1996b)

${ }^{4}$ Calculated from total plutonium. 
Table 5-5 compares the calculated mean concentrations for tank 241-B-204 sludge samples with those of tanks 241-B-201, 241-B-202, and 241-B-203. The HTCE estimates (Agnew 1996) identical sludge compositions for these four tanks.

Tank 241-B-201 was the first of the four tanks to receive waste. It received 224 waste in the first quarter of 1952; no other waste transfers were made into the tank. Tank 241-B-204 received 224 waste from the second quarter of 1952 to the third quarter of 1952 and B Plant flush water from the fourth quarter of 1952 to the first quarter of 1953 . These wastes cascaded from tank 241-B-204 to tank 241-B-203, then to tank 241-B-202, then to a crib.

Because tanks 241-B-204 and 241-B-203 are adjacent tanks in a cascade, they are expected to be similar in composition. Table 5-5 shows the waste compositions of the tanks are similar. Almost all analytes are within \pm 30 percent of each other. The limited number of analytes for tank 241-B-202 also compare well with tank 241-B-204. There is less similarity between tanks 241-B-201 and 241-B-204. Because tank 241-B-201 was the first to receive 224 waste, the composition of the 224 waste stream at startup might vary from later waste. Also, higher concentrations of aluminum, cesium, and strontium suggest that some first cycle decontamination waste and cladding waste may have been mixed in with the 224 waste that went to tank 241-B-201.

\subsection{TANK WASTE PROFILE}

The visual description of the sludge samples from both risers was soft, damp, and black thereby indicating possible homogeneity within the sludge layer. The color of the drainable liquid samples varied. Information on the vertical disposition of the tank contents was available from the TLM (see Figure 2-3), indicating that the waste consists of several inches of supernatant covering approximately 250 inches of sludge (composed of a single waste type). Thus, the waste sample description and the TLM indicate that a thin supernate layer overlies the sludge layer and that the sludge layer may be vertically homogeneous.

Because vertical profiles of the tank waste were taken from two widely-spaced risers, an analysis of variance (ANOVA) was conducted on the 1995 samples to determine whether there were horizontal and/or vertical variations in the analyte concentrations. These calculations were performed only for analytes that had half or more of their individual measurements above the detection limit. The ANOVA generates a p-value that is compared with a standard significance level $(\alpha=0.05)$. If a p-value is below 0.05 , there is sufficient evidence to conclude that the sample means are significantly different from each other and that the difference is not due to chance. However, if a p-value is above 0.05 , there is not sufficient evidence to conclude that the samples are significantly different from each other. 
Table 5-5. Comparison of B-200 Series Tank Compositions. (2 sheets)

\begin{tabular}{|c|c|c|c|c|}
\hline (2) & 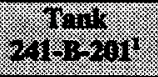 & 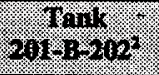 & \% & 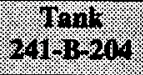 \\
\hline Chloride & 1,650 & nr & 861 & 693 \\
\hline Cyanide & 3.49 & nr & $<5.91$ & na \\
\hline Fluoride & 5,830 & $\mathrm{nr}$ & 7,790 & 7,080 \\
\hline Nitrate & 49,300 & $\mathrm{nr}$ & 63,900 & 52,900 \\
\hline Nitrite & 881 & $\mathrm{nr}$ & 730 & 694 \\
\hline Oxalate & na & $\mathrm{nr}$ & 2,020 & 1,710 \\
\hline Phosphate & 1,210 & $\mathrm{nr}$ & 3,850 & 3,620 \\
\hline Sulfate & 348 & $\mathrm{nr}$ & 702 & 648 \\
\hline Aluminum & 3,440 & $\mathbf{n r}$ & 52.1 & 65.3 \\
\hline Antimony & $<37.1$ & $\mathrm{nr}$ & $<18.9$ & $<26.9$ \\
\hline Arsenic & 59.5 & $\mathrm{nr}$ & $<53$ & $<44.8$ \\
\hline Barium & 86.4 & $\mathrm{nr}$ & 122 & $<199$ \\
\hline Beryllium & $<3.71$ & $\mathrm{nr}$ & $<1.56$ & $<2.24$ \\
\hline Bismuth & 94,500 & $\mathrm{nr}$ & 41,700 & 48,400 \\
\hline Boron & 70.5 & nr & 132 & 103 \\
\hline Cadmium & 4.81 & $\mathrm{nr}$ & $<1.57$ & $<2.26$ \\
\hline Calcium & 12,200 & $\mathrm{nr}$ & 222 & 305 \\
\hline Cerium & 69.6 & $\mathrm{nr}$ & 50.2 & 55.4 \\
\hline Chromium & 3,340 & $\mathrm{nr}$ & 3,080 & 3,240 \\
\hline Cobalt & 9.6 & $\mathrm{nr}$ & $<6.29$ & $<8.97$ \\
\hline Copper & 48.2 & $\mathrm{nr}$ & 6.99 & 22.1 \\
\hline Iron & 13,400 & $\mathrm{nr}$ & 4,410 & 3,810 \\
\hline Lanthanum & 15,100 & $\mathrm{nr}$ & 10,400 & 10,400 \\
\hline Lead & 1,360 & $\mathrm{nr}$ & $<473$ & $<1,070$ \\
\hline Magnesium & 1,510 & $\mathrm{nr}$ & 52.6 & 82.8 \\
\hline Manganese & 19,200 & nr & 14,200 & 14,800 \\
\hline Molybdenum & 19.1 & $\mathrm{nr}$ & $<15.7$ & $<22.4$ \\
\hline Neodymium & $<22.3$ & $\mathrm{nr}$ & $<31.5$ & $<44.8$ \\
\hline Nickel & 479 & $\mathrm{nr}$ & 183 & 234 \\
\hline Phosphorus & 5,450 & nr & 2,180 & 2,320 \\
\hline
\end{tabular}


WHC-SD-WM-ER-581 Rev. 0

Table 5-5. Comparison of B-200 Series Tank Compositions. (2 sheets)

\begin{tabular}{|c|c|c|c|c|}
\hline א. & 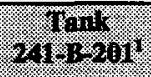 & 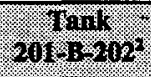 & $141 \sin ^{3}$ & $241 \mathrm{n}_{204}$ \\
\hline Potassium & 5,810 & $\mathbf{n r}$ & 5,120 & 5,780 \\
\hline Samarium & na & $\mathrm{nr}$ & $<31.4$ & $<44.8$ \\
\hline Selenium & 66.8 & $\mathrm{nr}$ & $<31.6$ & $<44.9$ \\
\hline Silicon & 20,200 & $\mathrm{nr}$ & 925 & 1,070 \\
\hline Silver & 12.3 & $\mathrm{nr}$ & 4.25 & $<5.56$ \\
\hline Sodium & 38,200 & $\overline{n r}$ & 29,000 & 26,200 \\
\hline Strontium & 923 & $\mathrm{nr}$ & 493 & 391 \\
\hline Sulfur & na & $\mathrm{nr}$ & 87.6 & 95 \\
\hline Thallium & $<371$ & $\mathrm{nr}$ & $<62.9$ & $<89.6$ \\
\hline Titanium & 285 & $\mathrm{nr}$ & $<3.74$ & $<6.98$ \\
\hline Uranium & 156 & $\mathrm{nr}$ & $<192$ & $<240$ \\
\hline Vanadium & 15.9 & $\mathrm{nr}$ & $<15.7$ & $<22.4$ \\
\hline Zinc & 217 & $\mathrm{nr}$ & 60.4 & 53.7 \\
\hline Zirconium & 10.7 & $\mathrm{nr}$ & $<3.15$ & $<4.52$ \\
\hline TIC & 2,090 & $\mathrm{nr}$ & 645 & na \\
\hline TOC & 518 & $\mathrm{nr}$ & 77.5 & na \\
\hline wt\% water & 60.7 & 61 & 75.8 & 77.1 \\
\hline${ }^{241} \mathrm{Am}$ & 0.031 & $\mathrm{nr}$ & na & $<0.0833$ \\
\hline${ }^{137} \mathrm{Cs}$ & 0.8 & $\mathrm{nr}$ & na & $<0.0314$ \\
\hline${ }^{60} \mathrm{Co}$ & 0.00196 & $\mathrm{nr}$ & na & $<0.0131$ \\
\hline${ }^{154} \mathrm{Eu}$ & 0.00438 & $\mathrm{nr}$ & na & $<0.0357$ \\
\hline${ }^{155} \mathrm{Eu}$ & 0.00328 & $\mathrm{nr}$ & na & $<0.0380$ \\
\hline Total alpha & 1.31 & 0.406 & 0.214 & 0.264 \\
\hline${ }^{90} \mathrm{Sr}$ & 2.09 & $\mathrm{nr}$ & na & 0.005 \\
\hline Density & 1.5 & approx 1.2 & 1.19 & 1.19 \\
\hline
\end{tabular}

Notes:

$\begin{array}{ll}\text { na } & =\text { not analyzed } \\ \mathbf{n r} & =\text { not reported }\end{array}$

'Heasler et al. (1994)

${ }^{2}$ Dougherty and Tran (1995)

3Jo et al. (1996) 
For drainable liquid samples, the results of the ANOVA indicated that none of the 17 analytes tested showed concentration differences between the two cores. The sludge results indicated that only three of 29 analytes tested had concentration differences between the two cores: bismuth, silicon, and density (with p-values of $0.0026,0.0034$, and 0.0006 , respectively). The test for vertical differences between the main segments was conducted only on the sludge samples because of the very limited vertical data from the drainable liquid results. Fourteen of the 29 analytes tested on the segment level, all metals, showed significant vertical concentration differences ( $p$-values are given in parentheses): aluminum $(<0.001)$, calcium $(<0.001)$, cerium $(0.033)$, chromium $(<0.001)$, lanthanum $(0.006)$, manganese $(<0.001)$, nickel $(<0.001)$, phosphorus $(<0.001)$, potassium $(<0.001)$, silicon $(<0.001)$, sodium $(0.003)$, strontium $(<0.001)$, sulfur $(0.002)$, and zinc $(<0.001)$. For these analytes showing vertical differences, no obvious general trend of increasing or decreasing analyte concentration as a function of depth was apparent. Of these 14 analytes, only seven have mean concentrations above 0.1 weight percent and only lanthanum, manganese, and sodium have mean concentrations above 1 weight percent. Subsegment concentrations ranged from 0.67 to 1.44 weight percent for lanthanum, 0.88 to 1.92 weight percent for manganese, and 2.24 to 2.90 weight percent for sodium.

In summary, horizontal concentration differences in the tank waste do not appear to exist. Vertically, the results of the statistical tests for the sludge layer indicate about half the analytes showed some differences among the segments, but no obvious trends appeared in analyte concentration as a function of the sample point in the tank.

\subsection{COMPARISON OF TRANSFER HISTORY WITH ANALYTICAL RESULTS}

Table 5-6 shows the HTCE predictions for the contents of tank 241-B-204 and Table 4-2 shows the mean sludge concentrations from the 1995 core sampling event. This comparison is for information purposes only, because the HTCE values have not been validated and should not be used for decisions affecting the waste in tank 241-B-204.

The HTCE and analytical values generally did not agree well. Eighteen analytes were compared. Only five analytes (potassium, chloride, nitrate, density, and percent water) exhibited RPDs less than 50 percent; of these, only one (chloride) exhibited an RPD less than 10 percent. Six analytes (calcium, chromium, manganese, nitrite, oxalate, and total alpha) exhibited RPDs greater than 150 percent. The RPDs for the remaining analytes were between these two extremes.

Table 5-6 shows the anion mean sludge concentrations as determined using a water-leach sample preparation and IC; therefore, the results for the anions forming insoluble compounds, fluoride and phosphate, will be biased low. If the phosphate and sulfate values, as calculated from ICP results, are compared with the HTCE predictions, the agreement for those two species is somewhat better. The phosphate and sulfate values, as determined by ICP, are in parentheses. 
Table 5-6. Comparison of HTCE Predictions with the 1995 Analytical Results for Tank 241-B-204. (2 sheets)

\begin{tabular}{|c|c|c|c|}
\hline (2) & 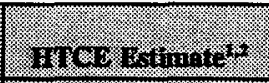 & 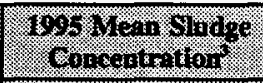 & 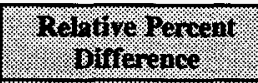 \\
\hline WHYWLS & 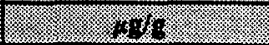 & 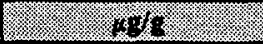 & (I) \\
\hline Bismuth & 9,170 & 48,400 & 136 \\
\hline Calcium & 7,100 & 305 & 184 \\
\hline Chromium & 130 & 3,240 & 185 \\
\hline Iron & 14,600 & 3,810 & 117 \\
\hline Lanthanum & 23,900 & 10,400 & 78.7 \\
\hline Manganese & 154 & 14,800 & 196 \\
\hline Nickel & 57.3 & 234 & 121 \\
\hline Potassium & 6,460 & 5,780 & 11.1 \\
\hline Sodium & 75,500 & 26,200 & 97.0 \\
\hline AHOMS & (2) & 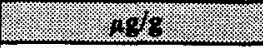 & (1) \\
\hline Chloride & 642 & 693 & 7.6 \\
\hline Fluoride & 28,100 & 7,080 & 120 \\
\hline Nitrate & 59,800 & 52,900 & 12.2 \\
\hline Nitrite & 0 & 694 & 200 \\
\hline Oxalate & 41,100 & 1,710 & 184 \\
\hline Phosphate $^{4}$ & 6,660 & $3,620(7110)$ & $\begin{array}{ll}59.1 & (6.5) \\
\end{array}$ \\
\hline Sulfate $^{4}$ & 93.7 & $\begin{array}{|ll|}648 & (284) \\
\end{array}$ & $150 \quad(101)$ \\
\hline RunOHUCH, &., $01 / 8$ & 1. $101 \mathrm{~g}$ & : \\
\hline Total alpha & $0.0100^{5}$ & 0.264 & 185 \\
\hline \multicolumn{3}{|c|}{ 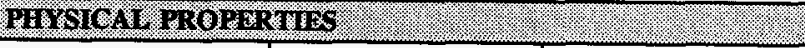 } & य. \\
\hline Bulk Density & $1.38 \mathrm{~g} / \mathrm{mL}$ & $1.19 \mathrm{~g} / \mathrm{mL}$ & 14.8 \\
\hline Percent Water & 55.5 & 77.1 & 32.6 \\
\hline
\end{tabular}

Notes:

'Agnew (1996)

2These data are not validated and are for comparison only.

${ }^{3}$ Sasali (1996b)

'Results in parentheses are calculated from ICP results.

${ }^{5}$ Calculated from total plutonium. 
The original process flowsheets from the lanthanum fluoride process were reviewed (Schneider 1951), and the source terms for the HDW definition of 224 (lanthanum fluoride) waste were examined. Results of this investigation provided plausible explanations for the large discrepancies observed for some analytes between the two data sets.

The HTCE underestimates the concentrations of bismuth, chromium, and manganese. Sodium bismuthate $\left(\mathrm{NaBiO}_{3}\right)$ was used as the primary oxidant during efforts to oxidize plutonium for separation from fission products. Sodium dichromate $\left(\mathrm{Na}_{2} \mathrm{Cr}_{2} \mathrm{O}_{7}\right)$ was used as a holding oxidant to stabilize oxidized plutonium. It is possible that the HDW did not take into account the metals used in these oxidizing compounds. According to Schneider (1951), potassium permanganate $\left(\mathrm{KMnO}_{4}\right)$ was determined to be an acceptable substitute for sodium dischromate in laboratory tests. However, the use of potassium permanganate is not specifically documented in the process flowsheets. The elevated levels of manganese in the waste samples strongly suggest that potassium permangante was used.

The HTCE overestimated the concentration of iron. It appears the HDW source term is biased high. From the process flowsheets, iron was not expected to be present in the quantities predicted by the HTCE. The discrepancy is probably caused by overestimating iron from corrosion.

It appeared that the HTCE also overestimated the concentration of sodium. Sodium and nitrate are the soluble chemical species present in the greatest quantities, and their charges can be expected to balance each other more or less. This prediction was born out in the analytical results evidenced by the change balance (see Section 5.1.3.2). Of the $1,290 \mu \mathrm{eq} / \mathrm{g}$ of positive charge, $1,140 \mu \mathrm{eq} / \mathrm{g}$ are attributed to sodium. Nitrate contributed $853 \mu \mathrm{eq} / \mathrm{g}$ to the anion total of $1,340 \mu \mathrm{eq} / \mathrm{g}$. A comparison of the sodium and nitrate values gives a ratio of 1.34. The HTCE values should be similarly distributed. However, a ratio of the HTCE sodium and nitrate predictions on a $\mu \mathrm{eq} / \mathrm{g}$ basis yields a value of 3.4. Consequently, it appears that a high bias in the HTCE sodium prediction does exist.

The reason for the poor agreement in fluoride concentrations is a deficiency in the analytical results. Because fluoride is measured by IC after a water digestion, only the soluble fluoride is measured. This does not compare with the HTCE fluoride value, which includes both soluble and insoluble fluoride.

The reason for the large HTCE prediction of oxalate is unknown. Organic material is not expected to be present in lanthanum fluoride in the high quantities estimated by the HTCE. In addition, the low radionuclide content of the waste is unlikely to produce large amounts of oxalate as an organic degradation product.

Finally, the HTCE prediction for weight percent water is too low. It is known from other samples of 224 waste (tanks 241-B-201, 241-B-202, 241-B-203, and 241-T-111) that the percent water in this waste type is 60 to 85 weight percent. 
In summary, the problems that occur when estimating the composition of the 224 waste are probably caused by documentation which does not describe the waste as it currently exists. A more accurate estimate of the 224 waste probably could be derived by examining the analytical results from all tanks containing 224 waste.

\subsection{EVALUATION OF PROGRAM REQUIREMENTS}

The two tank 241-B-204 core samples analyzed at the 222-S Laboratory were taken to meet the requirements of the safety screening DQO (Dukelow et al. 1995). This section discusses the requirements of the DQO and compares the analytical data to defined concentration limits.

\subsubsection{Safety Evaluation}

Data criteria, identified in the safety screening DQO, are used to assess the waste safety and to check for unidentified safety issues. The DQO requires a vertical profile of the tank waste from two widely-spaced risers. This requirement was met. Of the five primary analyses required by the DQO, three have decision criteria thresholds which, if exceeded, could warrant further investigation to ensure tank safety. These three analyses include DSC to measure the fuel content, a determination of the total alpha activity to evaluate the criticality potential, and a measurement of the flammability of the tank headspace vapors.

The safety screening DQO has established a notification limit of $-480 \mathrm{~J} / \mathrm{g}$ (dry weight basis) for exothermic reactions detected during the DSC analysis. No exothermic reactions were observed in any tank 241-B-204 samples.

The safety screening DQO decision threshold for criticality screening is $1 \mathrm{~g} / \mathrm{L}$ plutonium, or $41 \mu \mathrm{Ci} / \mathrm{g}$ as specified in the SAP. All results were well below the limit, with $0.496 \mu \mathrm{Ci} / \mathrm{g}$ being the largest single result. The highest 95 percent upper confidence level limit for these results was $1.00 \mu \mathrm{Ci} / \mathrm{g}$, far below the $\mathrm{DQO}$ limit.

The DQO notification limit for flammable gas concentration in the tank headspace is 25 percent of the lower flammability limit. Combustible gas meter readings taken at the time of sampling revealed the concentration of flammable gases to be 0 percent of the lower flammability limit.

In summary, the October 1995 core sampling event met all the requirements of the safety screening DQO. Table 5-7 lists the safety issues, the properties of concern and their notification limits, and the corresponding analytical results. 
Table 5-7. Safety Screening Data Quality Objective Decision Variables and Criteria.

\begin{tabular}{|l|l|l|l|}
\hline Ferrocyanide/Organics & Total fuel content & $-480 \mathrm{~J} / \mathrm{g}$ & $\begin{array}{l}\text { No exothermic } \\
\text { reactions }\end{array}$ \\
\hline Criticality & Total alpha activity & $41 \mu \mathrm{Ci} / \mathrm{g}$ & $0.264 \mu \mathrm{Ci} / \mathrm{g}$ \\
\hline Flammable gas & Flammable gas & $\begin{array}{l}25 \text { percent of the } \\
\text { lower flammability } \\
\text { limit }\end{array}$ & $\begin{array}{l}0 \text { percent of the } \\
\text { lower flammability } \\
\text { limit }\end{array}$ \\
\hline
\end{tabular}

Another factor in assessing tank waste safety is the heat generation and the temperature of the waste. Heat is generated in the tanks from radioactive decay. Radionuclides were only analyzed on one subsegment from one core. If the sludge is assumed to be homogeneous, a tank heat load of $<0.8 \mathrm{~W}(<3 \mathrm{Btu} / \mathrm{hr}$ ) is estimated from the analytical data. The HTCE prediction was zero (Brevick et al. 1994a), and the heat load, based on headspace temperature, was $47.2 \mathrm{~W}(161 \mathrm{Btu} / \mathrm{hr})$ (Kummerer 1994). These estimates are well below the $11,700 \mathrm{~W}(40,000 \mathrm{Btu} / \mathrm{hr})$ threshold differentiating high-heat from low-heat tanks (Bergmann 1991). Because an upper temperature limit has been exhibited (see Section 2.4.3), it may be concluded that any heat generated from radioactive sources throughout the year is dissipated.

Another safety related issue relates to the drying of wastes after they have been saltwell pumped. Tank 241-B-204 was not saltwell pumped, but it was interim stabilized in June 1984. The tank is not actively ventilated; therefore, the water loss from the waste is expected to be low. The sludge in tank $241-\mathrm{B}-204$ has a uniformly high water content (75 to 80 weight percent) which is similar to that measured in a sludge sample taken in 1978 prior to stabilization. There is a small amount of supernate $(1 \mathrm{kgal})$ in the tank. Liquid level readings indicate a decrease in the liquid level of approximately $1.3 \mathrm{~cm}(0.5 \mathrm{in}$.) or approximately $370 \mathrm{~L}$ (100 gal) since the tank was stabilized. 


\subsection{CONCLUSIONS AND RECOMMENDATIONS}

The waste in tank 241-B-204 has been sampled and analyzed according to the requirements listed in the Tank Safety Screening Data Quality Objective (Dukelow et al. 1995). The tank was core sampled using the push-mode method in October 1995. To assess tank safety, the safety screening DQO required analyses for energetics, weight percent water, density, total alpha activity, and the flammable gas concentration in the tank headspace. In addition, the SAP required the laboratory to perform ICP and IC analyses to determine the concentrations of lithium and bromide, indicators of contamination by the hydrostatic head fluid. The laboratory was also required to report all results generated by ICP and IC not just lithium and bromide (Sasaki 1996b). Finally, one subsegment was subjected to GEA and ${ }^{90} \mathrm{Sr}$ analysis to obtain information for the disposal of laboratory waste. All samples were analyzed at the Westinghouse Hanford Company 222-S Laboratory.

All analyses met the requirements of the safety screening DQO. No exothermic reactions were observed in the DSC analysis. Weight percent water was measured by TGA for the correction of the DSC results to a dry-weight basis; the overall mean (corrected for HHF contamination) was $77.1 \mathrm{wt} \%$ for the sludge and $89.1 \mathrm{wt} \%$ for the supernate. (Dry-weight corrections of the DSC results were not performed because no exotherms were observed.) The total alpha activity overall mean was $0.264 \mu \mathrm{Ci} / \mathrm{g}$, well below the DQO notification limit of $41 \mu \mathrm{Ci} / \mathrm{g}$. Finally, the concentration of flammable gas in the tank headspace was zero percent of the lower flammability limit. No quality assurance issues were identified that affected the interpretation of the analytical results. Based upon the decision criteria of the safety screening DQO, this tank may be categorized as "safe."

The heat load estimate based on analytical results was $<0.8 \mathrm{~W}(<3 \mathrm{Btu} / \mathrm{hr})$, the HTCE prediction was zero, and the heat load based on headspace temperature was $47.2 \mathrm{~W}$ (161 Btu/hr). Because the tank temperature is not continuously increasing, it may be concluded that any heat generated from radioactive sources throughout the year is dissipated.

Hydrostatic head fluid marked with a lithium bromide tracer was used during core sampling operations, and some contamination was detected in all samples; the maximum calculated HHF contamination was two weight-percent water. Corrections for the HHF contamination were applied to the weight-percent water measurements.

The results from the 1995 core samples show that the major cation constituents of the sludge are bismuth, chromium, iron, lanthanum, manganese, potassium, and sodium. Major anion constituents are fluoride, nitrate, and phosphate. Charge and mass balance calculations based on the 1995 sludge sample results indicate that the results appear to be reasonably selfconsistent. The charge balance yielded a value of 0.96 (ideal $=1$ ), and the mass balance was 97.4 percent (ideal $=100$ percent). Analysis of variance of the analytical data revealed only minor horizontal heterogeneity and statistically significant vertical heterogeneity in the sludge layer. 
Comparing the analytical results of the sludge from the 1995 core samples to 1978 historical results yielded mixed results. The sludge density and percent water values compare closely for the two data sets. However, many of the values for the chemical constituents, especially the major components, compare poorly. Because the details of the 1978 sampling event (such as riser number, sample depth, etc.) are unknown, and the 1995 results are an overall estimate for the entire tank, the two sets of samples may not be entirely comparable.

Between the HTCE estimate and the 1995 samples, only five analytes (potassium, chloride, nitrate, density, and percent water) compare favorably, and other major analytes (bismuth, sodium, fluoride, and phosphate) compare poorly. The poor fluoride and phosphate correlations may be due in part to the limitations of the water-leach sample preparation method used prior to the IC analysis of the 1995 samples. The water-leach sample preparation allows only a fraction of the insoluble fluoride and phosphate solids to be determined. The poor correlation of the other analytes between the HTCE and the 1995 core samples may indicate poor source term estimates for, or poor modeling of, those analytes in the HTCE. 


\subsection{REFERENCES}

Agnew, S. F., 1995, Hanford Defined Wastes: Chemical and Radionuclide Compositions, LA-UR-94-2657, Rev. 2, Los Alamos National Laboratory, Los Alamos, New Mexico.

Agnew, S. F., 1996, Hanford Tank Chemical and Radionuclide Inventories: HDW Model Rev. 3, LA-UR-96-858, Los Alamos National Laboratory, Los Alamos, New Mexico.

Agnew, S. F., P. Baca, R. Corbin, K. Jurgensen, and B. Young, 1995, Tank Layer Model (TLM) for the Northeast, Southwest, and Northwest Quadrants, LA-UR-94-4269, Rev. 1, Los Alamos National Laboratory, Los Alamos, New Mexico.

Agnew, S. F., P. Baca, R. Corbin, T. Duran, and K. Jurgensen, 1996, Waste Status and Transaction Record Summary for the Northeast Quadrant, WHC-SD-WM-TI-615, Rev. 1, Westinghouse Hanford Company, Richland, Washington.

Alstad, A. T., 1993, Riser Configuration Document for Single-Shell Waste Tanks, WHC-SD-RE-TI-053, Rev. 9, Westinghouse Hanford Company, Richland, Washington.

Bergmann, L. M., 1991, Single-Shell Tank Isolation Safety Analysis Report, WHC-SD-WM-SAR-006, Rev. 2, Westinghouse Hanford Company, Richland, Washington.

Brevick, C. H., L. A. Gaddis, W. W. Pickett, 1994a, Historical Tank Content Estimate for the Northeast Quadrant of the Hanford 200 East Area, WHC-SD-WM-ER-349, Rev. 0A, Westinghouse Hanford Company, Richland, Washington.

Brevick, C. H., L. A. Gaddis, E. Johnson, 1994b, Supporting Document for the Historical Tank Content Estimate for B Tank Farm, WHC-SD-WM-ER-310, Rev. 0, Westinghouse Hanford Company, Richland, Washington.

DeLorenzo, D. S., A. T. DiCenso, D. B. Hiller, K. W. Johnson, J. H. Rutherford, D.J. Smith, and B. C. Simpson, 1994, Tank Characterization Reference Guide, WHC-SD-WM-TI-648, Rev. 0, Westinghouse Hanford Company, Richland, Washington.

DOE, 1995, Hanford Analytical Services Quality Assurance Plan, DOE/RL-94-55, Rev. 2, U.S. Department of Energy, Richland, Washington.

Dougherty, L. F. and T. T. Tran, 1995, Tank Characterization Report for Single-Shell Tank 241-B-202, WHC-SD-WM-ER-371, Rev. 0, Westinghouse Hanford Company, Richland, Washington. 
Dukelow, G. T., J. W. Hunt, H. Babad, and J. E. Meacham, 1995, Tank Safety Screening Data Quality Objective, WHC-SD-WM-SP-004, Rev. 2, Westinghouse Hanford Company, Richland, Washington.

Ecology, EPA, and DOE, 1996, Hanford Federal Facility Agreement and Consent Order, as amended, Washington State Department of Ecology, U.S. Environmental Protection Agency, and U.S. Department of Energy, Olympia, Washington.

Hanlon, B. M., 1996, Waste Tank Summary Report for Month Ending January 31, 1996, WHC-EP-0182-94, Westinghouse Hanford Company, Richland, Washington.

Heasler, P. G., K. M. Remund, J. M. Tingey, D. B. Baird, and F. M. Ryan, 1994, Tank Characterization Report for Single-Shell Tank B-201, PNL-10100, Rev. 0, Pacific Northwest Laboratory, Richland, Washington.

Horton, J. E., 1978, "Characterization of 200 Series Tanks", Letter 60120-78-131 (to J. E. Mirabella, December 4), Atlantic Richfield Hanford Company, Richland, Washington.

Jo, J., L. C. Amato, and T. T. Tran, 1996, Tank Characterization Report for Single-Shell Tank 241-B-203, WHC-SD-WM-ER-587, Rev. 0, Westinghouse Hanford Company, Richland, Washington.

Kummerer, M., 1994, Topical Report on Heat Removal Characteristics of Waste Storage Tanks, WHC-SD-WM-SARR-010, Rev. 0, Westinghouse Hanford Company, Richland, Washington.

Leach, C. E. and S. M. Stahl, 1993, Hanford Site Tank Farm Facilities Interim Safety Basis, WHC-SD-WM-ISB-001, Rev. 0, Westinghouse Hanford Company, Richland, Washington.

Lipnicki, J., 1995, Waste Tank Risers Available for Sampling, WHC-SD-WM-TI-710, Rev. 2, Westinghouse Hanford Company, Richland, Washington.

Nuclear Regulatory Commission, 1988, Statistical Methods for Nuclear Materials Management, NUREG-CR-4604, PNL-5849, (C. A. Bennett and W. M. Bowen eds.), U.S. Government Printing Office, Washington, D.C.

Sasaki, L. M., 1995, Tank 241-B-204 Push Mode Core Sampling and Analysis Plan, WHC-SD-WM-TSAP-005, Rev. 1, Westinghouse Hanford Company, Richland, Washington.

Sasaki, L. M., 1996a, 45-Day Safety Screening Results for Tank 241-B-204, Push Mode Cores 112 and 114, WHC-SD-WM-DP-160, Rev. 0, Westinghouse Hanford Company, Richland, Washington. 
Sasaki, L. M., 1996b, Final Report for Tank 241-B-204, Push Mode Cores 112 and 114, WHC-SD-WM-DP-160, Rev. 1, Westinghouse Hanford Company, Richland, Washington.

Schneider, K. J., 1951, Flow Sheets and Flow Diagrams of Precipitation Separarions Process, HW-23043, General Electric Company, Richland, Washington.

Vitro Engineering Corporation, 1986, "Piping Waste Tank Isolation 241-B-204", Drawing No. H-2-73291, Rev. 2, ICF Kaiser Hanford, Richland, Washington. 
WHC-SD-WM-ER-581 Rev. 0

This page intentionally left blank. 
WHC-SD-WM-ER-581 Rev. 0

APPENDIX A

SAMPLE INFORMATION FOR 1995 CORE SAMPLING

A-1 
WHC-SD-WM-ER-581 Rev. 0

This page intentionally left blank. 


\section{APPENDIX A}

\section{SAMPLE INFORMATION FOR 1995 CORE SAMPLING}

\section{A.1 INTRODUCTION}

Appendix A contains the sample information for tank 241-B-204 push-mode cores 112 and 114 collected in October 1995 . Table A-1 shows the location of specific sample information in Tables A-2 through A-5. All data, except the core segment depths, are from Sasaki (1996b); core segment depths were obtained in a telephone conference from the core sampling cognizant engineer.

Table A-1. Sample Information Tables

\begin{tabular}{|c|c|}
\hline 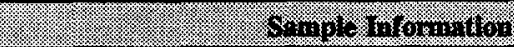 & (6aile \\
\hline $\begin{array}{l}\text { Core and segment number } \\
\text { Customer sample number } \\
\text { Sample depth } \\
\text { Drill string dose rate }\end{array}$ & Table A-2 \\
\hline $\begin{array}{l}\text { Core and segment number } \\
\text { Subsegment breakdown } \\
\text { Subsegment recovery (grams) }\end{array}$ & Table A-3 \\
\hline $\begin{array}{l}\text { Core and segment number } \\
\text { Laboratory sample number } \\
\text { Analyses performed }\end{array}$ & Table A-4 \\
\hline $\begin{array}{l}\text { Type of analysis } \\
\text { Analytical instrument } \\
\text { Sample preparation procedure number } \\
\text { Analytical procedure number }\end{array}$ & Table A-5 \\
\hline
\end{tabular}


Table A-2. Push-Mode Cores 112 and 114 Sample Information. (2 sheets)

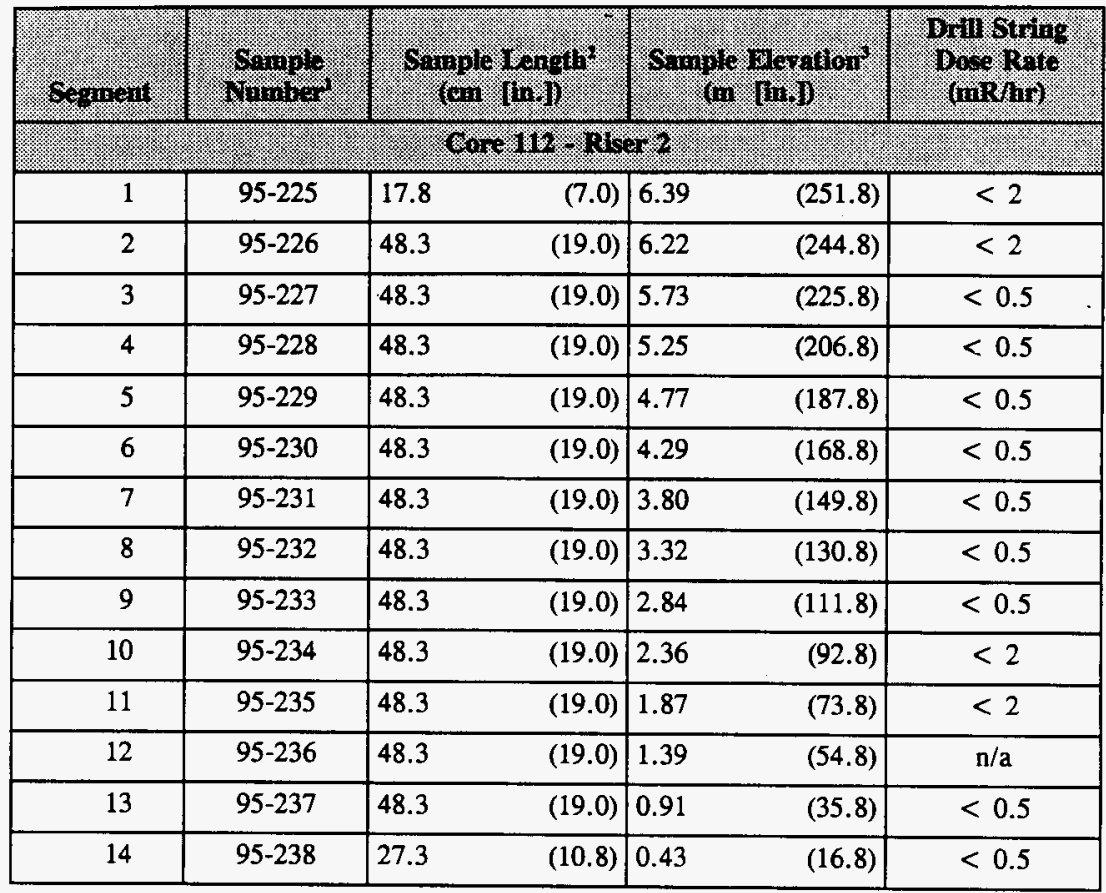


Table A-2. Push-Mode Cores 112 and 114 Sample Information. (2 sheets)

\begin{tabular}{|c|c|c|c|c|c|c|}
\hline 90 & (4) & $\sqrt{3}$ & $1 w^{2}$ & $\frac{10}{110}$ & $\frac{1310+3}{41}$ & 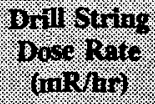 \\
\hline & & & 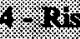 & $28 \%$ & & \\
\hline 1 & $95-246$ & 17.8 & (7.0) & 6.40 & $(252.0)$ & $<0.5$ \\
\hline 2 & $95-247$ & 48.3 & $(19.0)$ & 6.22 & $(245.0)$ & $<0.5$ \\
\hline 3 & $95-248$ & 48.3 & $(19.0)$ & 5.74 & $(226.0)$ & $<0.5$ \\
\hline 4 & $95-249$ & 48.3 & (19.0) & 5.26 & $(207.0)$ & $<0.5$ \\
\hline 5 & $95-250$ & 48.3 & $(19.0)$ & 4.78 & $(188.0)$ & $<0.5$ \\
\hline 6 & $95-251$ & 48.3 & $(19.0)$ & 4.29 & $(169.0)$ & $<0.5$ \\
\hline 7 & $95-252$ & 48.3 & $(19.0)$ & 3.81 & $(150.0)$ & $<0.5$ \\
\hline 8 & $95-253$ & 48.3 & $(19.0)$ & 3.33 & (131.0) & $<0.5$ \\
\hline 9 & $95-254$ & 48.3 & $(19.0)$ & 2.84 & $(112.0)$ & $<0.5$ \\
\hline 10 & $95-255$ & 48.3 & $(19.0)$ & 2.36 & $(93.0)$ & $<0.5$ \\
\hline 11 & $95-256$ & 48.3 & $(19.0)$ & 1.88 & $(74.0)$ & $<0.5$ \\
\hline 12 & $95-257$ & 48.3 & $(19.0)$ & 1.40 & $(55.0)$ & $<0.5$ \\
\hline 13 & $95-258$ & 48.3 & $(19.0)$ & 0.91 & $(36.0)$ & $<0.5$ \\
\hline 14 & $95-259$ & 27.9 & $(11.0)$ & 0.43 & $(17.0)$ & $<0.5$ \\
\hline \multicolumn{2}{|l|}{ Field blank } & \multicolumn{2}{|c|}{$\mathrm{n} / \mathrm{a}$} & \multicolumn{2}{|c|}{$\mathrm{n} / \mathrm{a}$} & $n / a$ \\
\hline \multicolumn{2}{|c|}{ Hydrostatic head fluid blank } & \multicolumn{2}{|c|}{$\mathrm{n} / \mathrm{a}$} & \multicolumn{2}{|c|}{$\mathrm{n} / \mathrm{a}$} & $\mathrm{n} / \mathrm{a}$ \\
\hline
\end{tabular}

Notes:

$\mathbf{n} / \mathbf{a}=$ not available or not applicable

'Customer sample number

2"Sample length" is the distance the drill string was advanced for that core segment and is not necessarily the same as the length of the recovered core.

${ }^{3}$ Sample elevation is measured from the bottom centerline of the tank to the top of the segment; these values are approximate, and the uncertainty on these values is unknown. 
Table A-3. Subsampling Scheme, Recovery, and Sample Description. (3 sheets)

\begin{tabular}{|c|c|c|c|}
\hline$T_{6}, 31,12$ & 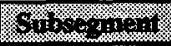 & 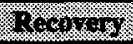 & Bencelplon \\
\hline \multicolumn{4}{|c|}{ 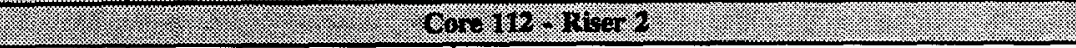 } \\
\hline \multirow[t]{2}{*}{1} & $\mathrm{DL}$ & $150.1 \mathrm{~g}$ & \multirow{2}{*}{$\begin{array}{l}\text { DL was clear and yellow. No solids present. } \\
\text { Estimated sample volume was } 150 \mathrm{~mL} \text {. }\end{array}$} \\
\hline & LL & $<5 \mathrm{~mL}$ & \\
\hline \multirow[t]{3}{*}{2} & Whole & $40.2 \mathrm{~g}$ & \multirow{3}{*}{$\begin{array}{l}\text { DL was opaque and dark gray/black. Three to four } \\
\text { in. of solids extruded. Solids were wet and black } \\
\text { and did not retain their shape. No facies present. }\end{array}$} \\
\hline & $\mathrm{DL}$ & $125.6 \mathrm{~g}$ & \\
\hline & $\mathbf{L L}$ & $122.5 \mathrm{~g}$ & \\
\hline \multirow[t]{3}{*}{3} & Upper $1 / 2$ & $167.2 \mathrm{~g}$ & \multirow{3}{*}{$\begin{array}{l}19 \text { in. of solids were extruded. Solids were a } \\
\text { black, damp, and soft sludge that retained its shape. } \\
\text { The top in. of upper } 1 / 2 \text { contained a slurry. No } \\
\text { facies or DL. }\end{array}$} \\
\hline & Lower $1 / 2$ & $179.9 \mathrm{~g}$ & \\
\hline & LL & $<5 \mathrm{~mL}$ & \\
\hline \multirow[t]{3}{*}{4} & Upper $1 / 2$ & $183.3 \mathrm{~g}$ & \multirow{3}{*}{$\begin{array}{l}19 \text { in. of solids were extruded. Solids were a } \\
\text { black, damp, and soft sludge that retained its shape. } \\
\text { No facies or DL. }\end{array}$} \\
\hline & Lower $1 / 2$ & $186.1 \mathrm{~g}$ & \\
\hline & LL & $<5 \mathrm{~mL}$ & \\
\hline \multirow[t]{2}{*}{5} & Upper $1 / 2$ & $176.5 \mathrm{~g}$ & \multirow{2}{*}{$\begin{array}{l}19 \mathrm{in} \text {. of solids were extruded. Solids were a } \\
\text { black, damp, and soft sludge that retained its shape. } \\
\text { No facies, DL, or LL. }\end{array}$} \\
\hline & Lower $1 / 2$ & $185.4 \mathrm{~g}$ & \\
\hline \multirow[t]{2}{*}{6} & Upper $1 / 2$ & $188.3 \mathrm{~g}$ & \multirow{2}{*}{$\begin{array}{l}19 \text { in. of solids were extruded. Solids were a } \\
\text { black, damp, and soft sludge that retained its shape. } \\
\text { No facies, DL, or LL. }\end{array}$} \\
\hline & Lower $1 / 2$ & $175.0 \mathrm{~g}$ & \\
\hline \multirow[t]{2}{*}{7} & Upper $1 / 2$ & $179.6 \mathrm{~g}$ & \multirow{2}{*}{$\begin{array}{l}19 \mathrm{in} \text {. of solids were extruded. Solids were a } \\
\text { black, damp, and soft sludge that retained its shape. } \\
\text { No facies, DL, or LL. }\end{array}$} \\
\hline & Lower $1 / 2$ & $177.4 \mathrm{~g}$ & \\
\hline \multirow[t]{3}{*}{8} & Upper $1 / 2$ & $182.8 \mathrm{~g}$ & \multirow{3}{*}{$\begin{array}{l}19 \text { in. of solids were extruded. Solids were a } \\
\text { black, damp, and soft sludge that retained its shape. } \\
\text { No facies or DL. }\end{array}$} \\
\hline & Lower $1 / 2$ & $184.0 \mathrm{~g}$ & \\
\hline & $\mathbf{L L}$ & $7.4 \mathrm{~g}$ & \\
\hline \multirow[t]{3}{*}{9} & Upper $1 / 2$ & $190.2 \mathrm{~g}$ & \multirow{3}{*}{$\begin{array}{l}19 \text { in. of solids were extruded. Solids were a } \\
\text { black, damp, and soft sludge that retained its shape. } \\
\text { No facies or DL. }\end{array}$} \\
\hline & Lower $1 / 2$ & $176.9 \mathrm{~g}$ & \\
\hline & $\mathrm{LL}$ & $<5 \mathrm{~mL}$ & \\
\hline \multirow[t]{3}{*}{10} & Upper $1 / 2$ & $206.4 \mathrm{~g}$ & \multirow{3}{*}{$\begin{array}{l}19 \text { in. of solids were extruded. Solids were a } \\
\text { black, damp, and soft sludge that retained its shape. } \\
\text { No facies or DL. }\end{array}$} \\
\hline & Lower $1 / 2$ & $161.8 \mathrm{~g}$ & \\
\hline & $\mathrm{LL}$ & $<5 \mathrm{~mL}$ & \\
\hline \multirow[t]{3}{*}{11} & Upper $1 / 2$ & $194.5 \mathrm{~g}$ & \multirow{3}{*}{$\begin{array}{l}19 \text { in. of solids were extruded. Solids were a } \\
\text { black, damp, and soft sludge that retained its shape. } \\
\text { No facies or DL. }\end{array}$} \\
\hline & Lower $1 / 2$ & $183.6 \mathrm{~g}$ & \\
\hline & LL & $<5 \mathrm{~mL}$ & \\
\hline
\end{tabular}


Table A-3. Subsampling Scheme, Recovery, and Sample Description. (3 sheets)

\begin{tabular}{|c|c|c|c|}
\hline $7+3110114$ & $(416,-3116112$ & $1+3-438)=8$ & $30=2 \times 91403$ \\
\hline \multicolumn{4}{|c|}{ : } \\
\hline \multirow[t]{3}{*}{12} & Upper $1 / 2$ & $192.0 \mathrm{~g}$ & \multirow{3}{*}{$\begin{array}{l}19 \text { in. of solids were extruded. Solids were a } \\
\text { black, damp, and soft sludge that retained its shape. } \\
\text { No facies or DL. }\end{array}$} \\
\hline & Lower $1 / 2$ & $180.7 \mathrm{~g}$ & \\
\hline & LL & $<5 \mathrm{~mL}$ & \\
\hline \multirow[t]{3}{*}{13} & Upper $1 / 2$ & $184.0 \mathrm{~g}$ & \multirow{3}{*}{$\begin{array}{l}19 \text { in. of solids were extruded. Solids were a } \\
\text { black, damp, and soft sludge that retained its shape. } \\
\text { No facies or DL. }\end{array}$} \\
\hline & Lower $1 / 2$ & $191.7 \mathrm{~g}$ & \\
\hline & $\mathrm{LL}$ & $<5 \mathrm{~mL}$ & \\
\hline \multirow[t]{3}{*}{14} & Upper $1 / 2$ & $197.4 \mathrm{~g}$ & \multirow{3}{*}{$\begin{array}{l}19 \mathrm{in.} \text { of solids were extruded. Solids were a } \\
\text { black, damp, and soft sludge that retained its shape. } \\
\text { No facies or DL. }\end{array}$} \\
\hline & Lower $1 / 2$ & $169.1 \mathrm{~g}$ & \\
\hline & LL & $<5 \mathrm{~mL}$ & \\
\hline & (3) & & 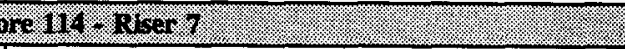 \\
\hline \multirow[t]{2}{*}{1} & $\mathrm{DL}$ & $125.5 \mathrm{~g}$ & \multirow{2}{*}{$\begin{array}{l}\text { DL was opaque and brownish gray. No solids } \\
\text { were present. Estimated sample volume was } 130 \\
\text { mL. }\end{array}$} \\
\hline & LL & $<20 \mathrm{~mL}$ & \\
\hline \multirow[t]{3}{*}{2} & Upper $1 / 2$ & $143.8 \mathrm{~g}$ & \multirow{3}{*}{$\begin{array}{l}19 \text { in. of solids were extruded. Solids were a } \\
\text { black, damp, and soft sludge. Bottom } 15 \text { in. } \\
\text { retained its shape but slumped slightly. Top } 4 \text { in. } \\
\text { did not keep its shape. No facies or DL. }\end{array}$} \\
\hline & Lower $1 / 2$ & $178.7 \mathrm{~g}$ & \\
\hline & $\mathrm{LL}$ & $<5 \mathrm{~mL}$ & \\
\hline \multirow[t]{3}{*}{3} & Upper $1 / 2$ & $163.3 \mathrm{~g}$ & \multirow{3}{*}{$\begin{array}{l}19 \text { in. of solids were extruded. Solids were a } \\
\text { black, damp, and soft sludge that retained its shape. } \\
\text { No facies or DL. }\end{array}$} \\
\hline & Lower $1 / 2$ & $162.0 \mathrm{~g}$ & \\
\hline & $\mathrm{LL}$ & $<10 \mathrm{~mL}$ & \\
\hline \multirow[t]{2}{*}{4} & Upper $1 / 2$ & $171.9 \mathrm{~g}$ & \multirow{2}{*}{$\begin{array}{l}19 \text { in. of solids were extruded. Solids were a } \\
\text { black, damp, and soft sludge that retained its shape. } \\
\text { No facies or DL. }\end{array}$} \\
\hline & Lower $1 / 2$ & $172.8 \mathrm{~g}$ & \\
\hline \multirow[t]{3}{*}{5} & Upper $1 / 2$ & $178.5 \mathrm{~g}$ & \multirow{3}{*}{$\begin{array}{l}19 \text { in. of solids were extruded. Solids were a } \\
\text { black, damp, and soft sludge that retained its shape. } \\
\text { No facies or DL. }\end{array}$} \\
\hline & Lower $1 / 2$ & $165.3 \mathrm{~g}$ & \\
\hline & $\mathrm{LL}$ & $<10 \mathrm{~mL}$ & \\
\hline \multirow[t]{3}{*}{6} & Upper $1 / 2$ & $163.5 \mathrm{~g}$ & \multirow{3}{*}{$\begin{array}{l}19 \text { in. of solids were extruded. Solids were a } \\
\text { black, damp, and soft sludge that retained its shape } \\
\text { for most segments. Top } 2-3 \text { in. were softer and did } \\
\text { not retain their shape. No facies or DL. }\end{array}$} \\
\hline & Lower $1 / 2$ & $176.0 \mathrm{~g}$ & \\
\hline & $\mathrm{LL}$ & $<10 \mathrm{~mL}$ & \\
\hline \multirow[t]{3}{*}{7} & Upper $1 / 2$ & $189.3 \mathrm{~g}$ & \multirow{3}{*}{$\begin{array}{l}19 \text { in. of solids were extruded. Solids were a } \\
\text { black, damp, and soft sludge that retained its shape. } \\
\text { No facies or DL. }\end{array}$} \\
\hline & Lower $1 / 2$ & $167.0 \mathrm{~g}$ & \\
\hline & $\mathrm{LL}$ & $<20 \mathrm{~mL}$ & \\
\hline
\end{tabular}


Table A-3. Subsampling Scheme, Recovery, and Sample Description. (3 sheets)

\begin{tabular}{|c|c|c|c|}
\hline $4=211=11$ & 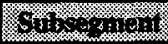 & 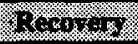 & $B=\alpha+x+10 n$ \\
\hline & & \multicolumn{2}{|c|}{ 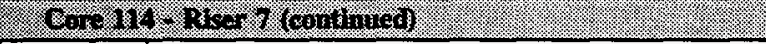 } \\
\hline \multirow[t]{3}{*}{8} & Upper $1 / 2$ & $175.5 \mathrm{~g}$ & \multirow{3}{*}{$\begin{array}{l}19 \text { in. of solids were extruded. Solids were a } \\
\text { black, damp, and soft sludge that retained its shape. } \\
\text { No facies or DL. }\end{array}$} \\
\hline & Lower $1 / 2$ & $183.5 \mathrm{~g}$ & \\
\hline & LL & $<20 \mathrm{~mL}$ & \\
\hline \multirow[t]{3}{*}{9} & Upper $1 / 2$ & $172.0 \mathrm{~g}$ & \multirow{3}{*}{$\begin{array}{l}19 \text { in. of solids were extruded. Solids were a } \\
\text { black, damp, and soft sludge that retained its shape. } \\
\text { No facies or DL. }\end{array}$} \\
\hline & Lower $1 / 2$ & $191.1 \mathrm{~g}$ & \\
\hline & $\mathrm{LL}$ & $<20 \mathrm{~mL}$ & \\
\hline \multirow[t]{3}{*}{10} & Upper $1 / 2$ & $179.1 \mathrm{~g}$ & \multirow{3}{*}{$\begin{array}{l}19 \text { in. of solids were extruded. Solids were a } \\
\text { black, damp, and soft sludge that retained its shape. } \\
\text { No facies or DL. }\end{array}$} \\
\hline & Lower $1 / 2$ & $186.5 \mathrm{~g}$ & \\
\hline & LL & $<20 \mathrm{~mL}$ & \\
\hline \multirow[t]{3}{*}{11} & Upper $1 / 2$ & $188.9 \mathrm{~g}$ & \multirow{3}{*}{$\begin{array}{l}19 \text { in. of solids were extruded. Solids were a } \\
\text { black, damp, and soft sludge that retained its shape. } \\
\text { No facies or DL. }\end{array}$} \\
\hline & Lower $1 / 2$ & $176.0 \mathrm{~g}$ & \\
\hline & $\mathrm{LL}$ & $<20 \mathrm{~mL}$ & \\
\hline \multirow[t]{3}{*}{12} & Upper $1 / 2$ & $165.6 \mathrm{~g}$ & \multirow{3}{*}{$\begin{array}{l}19 \text { in. of solids were extruded. Solids were a } \\
\text { black, damp, and soft sludge that retained its shape. } \\
\text { No facies or DL. }\end{array}$} \\
\hline & Lower $1 / 2$ & $198.6 \mathrm{~g}$ & \\
\hline & LL & $<20 \mathrm{~mL}$ & \\
\hline \multirow[t]{3}{*}{13} & Upper $1 / 2$ & $171.3 \mathrm{~g}$ & \multirow{3}{*}{$\begin{array}{l}19 \text { in. of solids were extruded. Solids were a } \\
\text { black, damp, and soft sludge that retained its shape. } \\
\text { No facies or DL. }\end{array}$} \\
\hline & Lower $1 / 2$ & $198.2 \mathrm{~g}$ & \\
\hline & $\mathrm{LL}$ & $<5 \mathrm{~mL}$ & \\
\hline \multirow[t]{3}{*}{14} & Upper $1 / 2$ & $195.5 \mathrm{~g}$ & \multirow{3}{*}{$\begin{array}{l}19 \text { in. of solids were extruded. Solids were a } \\
\text { black, damp, and soft sludge that retained its shape. } \\
\text { No facies or DL. }\end{array}$} \\
\hline & Lower $1 / 2$ & $176.3 \mathrm{~g}$ & \\
\hline & $\mathrm{LL}$ & $<5 \mathrm{~mL}$ & \\
\hline \multicolumn{2}{|c|}{ Field blank } & $213.6 \mathrm{~g}$ & Clear liquid. No solids. No liner liquid. \\
\hline \multicolumn{2}{|c|}{$\begin{array}{l}\text { Hydrostatic head fluid } \\
\text { blank }\end{array}$} & $\mathrm{n} / \mathrm{a}$ & $\mathrm{n} / \mathrm{a}$ \\
\hline
\end{tabular}

Note:
$\mathrm{DL}=$ drainable liquid
$L I=$ liner liquid
$\mathbf{n} / \mathbf{a}=$ not applicable 
Table A-4. Summary of Samples and Analyses. (8 sheets)

\begin{tabular}{|c|c|c|c|}
\hline 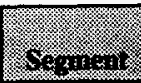 & 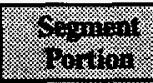 & Yingerits & inalyes \\
\hline \multicolumn{4}{|c|}{ (1) } \\
\hline 1 & DL & 2976 & $\begin{array}{l}\text { TGA, Specific gravity, DSC, ICP, IC, } \\
\text { Alpha }\end{array}$ \\
\hline \multirow[t]{3}{*}{2} & Whole & $\begin{array}{l}2977 \\
2979 \\
2980 \\
2981 \\
2987 \\
\end{array}$ & $\begin{array}{l}\text { Bulk density } \\
\text { TGA, DSC } \\
\text { ICP } \\
\text { IC } \\
\text { Alpha }\end{array}$ \\
\hline & DL & 2984 & $\begin{array}{l}\text { TGA, Specific gravity, DSC, ICP, IC, } \\
\text { Alpha }\end{array}$ \\
\hline & $L L$ & 2986 & $\begin{array}{l}\text { TGA, Specific gravity, DSC, ICP, IC, } \\
\text { Alpha }\end{array}$ \\
\hline \multirow[t]{2}{*}{3} & Upper $1 / 2$ & $\begin{array}{l}2988 \\
3037 \\
3061 \\
3085\end{array}$ & $\begin{array}{l}\text { Bulk density } \\
\text { TGA, DSC } \\
\text { ICP } \\
\text { IC }\end{array}$ \\
\hline & Lower $1 / 2$ & $\begin{array}{l}2989 \\
3038 \\
3062 \\
3086 \\
3109 \\
\end{array}$ & $\begin{array}{l}\text { Bulk density } \\
\text { TGA, DSC } \\
\text { ICP } \\
\text { IC } \\
\text { Alpha } \\
\end{array}$ \\
\hline \multirow[t]{2}{*}{4} & Upper $1 / 2$ & \begin{tabular}{|l|}
2991 \\
3039 \\
3063 \\
3087
\end{tabular} & $\begin{array}{l}\text { Bulk density } \\
\text { TGA, DSC } \\
\text { ICP } \\
\text { IC }\end{array}$ \\
\hline & Lower $1 / 2$ & $\begin{array}{l}2992 \\
3040 \\
3064 \\
3088 \\
3110\end{array}$ & $\begin{array}{l}\text { Bulk density } \\
\text { TGA, DSC } \\
\text { ICP } \\
\text { IC } \\
\text { Alpha }\end{array}$ \\
\hline
\end{tabular}


Table A-4. Summary of Samples and Analyses. (8 sheets)

\begin{tabular}{|c|c|c|c|}
\hline 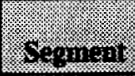 & 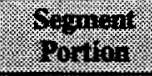 & Ualorere: & ( \\
\hline \multicolumn{4}{|c|}{ 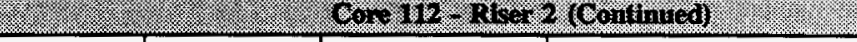 } \\
\hline \multirow[t]{2}{*}{5} & Upper $1 / 2$ & $\begin{array}{l}2993 \\
3041 \\
3065 \\
3066 \\
3089 \\
\end{array}$ & $\begin{array}{l}\text { Bulk density } \\
\text { TGA, DSC } \\
\text { ICP } \\
\text { ICP } \\
\text { IC } \\
\end{array}$ \\
\hline & Lower $1 / 2$ & $\begin{array}{l}2994 \\
3042 \\
3090 \\
3111\end{array}$ & $\begin{array}{l}\text { Bulk density } \\
\text { TGA, DSC } \\
\text { IC } \\
\text { Alpha }\end{array}$ \\
\hline \multirow[t]{2}{*}{6} & Upper $1 / 2$ & $\begin{array}{l}2995 \\
3043 \\
3067 \\
3091 \\
\end{array}$ & $\begin{array}{l}\text { Bulk density } \\
\text { TGA, DSC } \\
\text { ICP } \\
\text { IC } \\
\end{array}$ \\
\hline & Lower $1 / 2$ & \begin{tabular}{|l|}
2996 \\
3044 \\
3068 \\
3092 \\
3112 \\
\end{tabular} & $\begin{array}{l}\text { Bulk density } \\
\text { TGA, DSC } \\
\text { ICP } \\
\text { IC } \\
\text { Alpha } \\
\end{array}$ \\
\hline \multirow[t]{2}{*}{7} & Upper $1 / 2$ & $\begin{array}{l}2997 \\
3045 \\
3069 \\
3093 \\
\end{array}$ & $\begin{array}{l}\text { Bulk density } \\
\text { TGA, DSC } \\
\text { ICP } \\
\text { IC } \\
\end{array}$ \\
\hline & Lower $1 / 2$ & $\begin{array}{l}2998 \\
3046 \\
3070 \\
3094 \\
3113 \\
\end{array}$ & $\begin{array}{l}\text { Bulk density } \\
\text { TGA, DSC } \\
\text { ICP } \\
\text { IC } \\
\text { Alpha }\end{array}$ \\
\hline \multirow[t]{2}{*}{8} & Upper $1 / 2$ & $\begin{array}{l}2999 \\
3047 \\
3071 \\
3095 \\
\end{array}$ & $\begin{array}{l}\text { Bulk density } \\
\text { TGA, DSC } \\
\text { ICP } \\
\text { IC }\end{array}$ \\
\hline & Lower $1 / 2$ & $\begin{array}{l}3000 \\
3048 \\
3072 \\
3096 \\
3114 \\
\end{array}$ & $\begin{array}{l}\text { Bulk density } \\
\text { TGA, DSC } \\
\text { ICP } \\
\text { IC } \\
\text { Alpha }\end{array}$ \\
\hline
\end{tabular}


WHC-SD-WM-ER-581 Rev. 0

Table A-4. Summary of Samples and Analyses. (8 sheets)

\begin{tabular}{|c|c|c|c|}
\hline Cocuniterit & 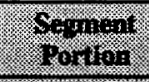 & 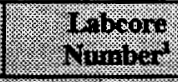 & t. \\
\hline \multicolumn{4}{|c|}{ 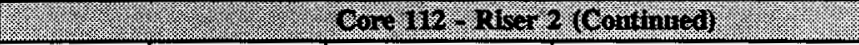 } \\
\hline \multirow[t]{2}{*}{9} & Upper $1 / 2$ & $\begin{array}{l}3001 \\
3049 \\
3073 \\
3097 \\
\end{array}$ & $\begin{array}{l}\text { Bulk density } \\
\text { TGA, DSC } \\
\text { ICP } \\
\text { IC }\end{array}$ \\
\hline & Lower $1 / 2$ & $\begin{array}{l}3002 \\
3050 \\
3074 \\
3098 \\
3115 \\
\end{array}$ & $\begin{array}{l}\text { Bulk density } \\
\text { TGA, DSC } \\
\text { ICP } \\
\text { IC } \\
\text { Alpha }\end{array}$ \\
\hline \multirow[t]{2}{*}{10} & Upper $1 / 2$ & $\begin{array}{l}3003 \\
3051 \\
3075 \\
3099\end{array}$ & $\begin{array}{l}\text { Bulk density } \\
\text { TGA, DSC } \\
\text { ICP } \\
\text { IC }\end{array}$ \\
\hline & Lower $1 / 2$ & $\begin{array}{l}3004 \\
3052 \\
3076 \\
3100 \\
3116 \\
\end{array}$ & \begin{tabular}{|l} 
Bulk density \\
TGA, DSC \\
ICP \\
IC \\
Alpha \\
\end{tabular} \\
\hline \multirow[t]{2}{*}{11} & Upper $1 / 2$ & $\begin{array}{l}3005 \\
3053 \\
3077 \\
3101 \\
\end{array}$ & \begin{tabular}{|l} 
Bulk density \\
TGA, DSC \\
ICP \\
IC \\
\end{tabular} \\
\hline & Lower $1 / 2$ & $\begin{array}{l}3006 \\
3054 \\
3078 \\
3102 \\
3117 \\
\end{array}$ & $\begin{array}{l}\text { Bulk density } \\
\text { TGA, DSC } \\
\text { ICP } \\
\text { IC } \\
\text { Alpha } \\
\end{array}$ \\
\hline \multirow[t]{2}{*}{12} & Upper $1 / 2$ & \begin{tabular}{|l}
3007 \\
3055 \\
3079 \\
3103 \\
\end{tabular} & $\begin{array}{l}\text { Bulk density } \\
\text { TGA, DSC } \\
\text { ICP } \\
\text { IC } \\
\end{array}$ \\
\hline & Lower $1 / 2$ & \begin{tabular}{|l|}
3008 \\
3056 \\
3080 \\
3104 \\
3118
\end{tabular} & $\begin{array}{l}\text { Bulk density } \\
\text { TGA, DSC } \\
\text { ICP } \\
\text { IC } \\
\text { Alpha }\end{array}$ \\
\hline
\end{tabular}


Table A-4. Summary of Samples and Analyses. (8 sheets)

\begin{tabular}{|c|c|c|c|}
\hline (3.sing & 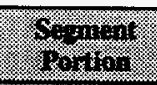 & 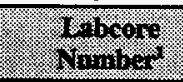 & (3. \\
\hline \multicolumn{4}{|c|}{ 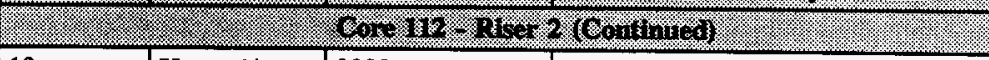 } \\
\hline \multirow[t]{2}{*}{13} & Upper $1 / 2$ & $\begin{array}{l}3009 \\
3057 \\
3081 \\
3105\end{array}$ & $\begin{array}{l}\text { Bulk density } \\
\text { TGA, DSC } \\
\text { ICP } \\
\text { IC } \\
\end{array}$ \\
\hline & Lower $1 / 2$ & \begin{tabular}{|l|}
3010 \\
3058 \\
3082 \\
3106 \\
3119 \\
\end{tabular} & $\begin{array}{l}\text { Bulk density } \\
\text { TGA, DSC } \\
\text { ICP } \\
\text { IC } \\
\text { Alpha } \\
\end{array}$ \\
\hline \multirow[t]{2}{*}{14} & Upper $1 / 2$ & \begin{tabular}{|l|}
3011 \\
3059 \\
3083 \\
3107 \\
\end{tabular} & $\begin{array}{l}\text { Bulk density } \\
\text { TGA, DSC } \\
\text { ICP } \\
\text { IC } \\
\end{array}$ \\
\hline & Lower $1 / 2$ & $\begin{array}{l}3012 \\
3060 \\
3084 \\
3108 \\
3120\end{array}$ & \begin{tabular}{|l|} 
Bulk density \\
TGA, DSC \\
ICP \\
IC \\
Alpha, Americium, Cobalt, Cesium, \\
Europium, Strontium \\
\end{tabular} \\
\hline \multicolumn{4}{|c|}{ 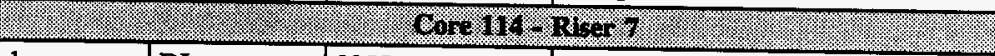 } \\
\hline 1 & DL & 3255 & TGA, DSC, ICP, IC, Alpha \\
\hline \multirow[t]{2}{*}{2} & Upper $1 / 2$ & $\begin{array}{l}3202 \\
3256 \\
3282 \\
3308 \\
\end{array}$ & $\begin{array}{l}\text { Bulk density } \\
\text { TGA, DSC } \\
\text { ICP } \\
\text { IC } \\
\end{array}$ \\
\hline & Lower $1 / 2$ & $\begin{array}{l}3203 \\
3257 \\
3283 \\
3309 \\
3334 \\
\end{array}$ & $\begin{array}{l}\text { Bulk density } \\
\text { TGA, DSC } \\
\text { ICP } \\
\text { IC } \\
\text { Alpha } \\
\end{array}$ \\
\hline
\end{tabular}


WHC-SD-WM-ER-581 Rev. 0

Table A-4. Summary of Samples and Analyses. (8 sheets)

\begin{tabular}{|c|c|c|c|}
\hline 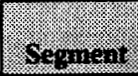 & 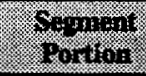 & 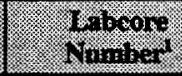 & (2): \\
\hline (3) & (1) & $6,20,164=2 y$ & 7 (Continued) \\
\hline \multirow[t]{2}{*}{3} & Upper $1 / 2$ & $\begin{array}{l}3204 \\
3258 \\
3284 \\
3310\end{array}$ & $\begin{array}{l}\text { Bulk density } \\
\text { TGA, DSC } \\
\text { ICP } \\
\text { IC }\end{array}$ \\
\hline & Lower $1 / 2$ & $\begin{array}{l}3205 \\
3259 \\
3285 \\
3311 \\
3335\end{array}$ & $\begin{array}{l}\text { Bulk density } \\
\text { TGA, DSC } \\
\text { ICP } \\
\text { IC } \\
\text { Alpha }\end{array}$ \\
\hline \multirow[t]{2}{*}{4} & Upper $1 / 2$ & $\begin{array}{l}3206 \\
3260 \\
3286 \\
3312 \\
\end{array}$ & $\begin{array}{l}\text { Bulk density } \\
\text { TGA, DSC } \\
\text { ICP } \\
\text { IC } \\
\end{array}$ \\
\hline & Lower $1 / 2$ & $\begin{array}{l}3207 \\
3261 \\
3287 \\
3313 \\
3336 \\
\end{array}$ & $\begin{array}{l}\text { Bulk density } \\
\text { TGA, DSC } \\
\text { ICP } \\
\text { IC } \\
\text { Alpha }\end{array}$ \\
\hline \multirow[t]{2}{*}{5} & Upper $1 / 2$ & $\begin{array}{l}3208 \\
3262 \\
3288 \\
3314 \\
\end{array}$ & $\begin{array}{l}\text { Bulk density } \\
\text { TGA, DSC } \\
\text { ICP } \\
\text { IC } \\
\end{array}$ \\
\hline & Lower $1 / 2$ & $\begin{array}{l}3209 \\
3263 \\
3289 \\
3315 \\
3337 \\
\end{array}$ & $\begin{array}{l}\text { Bulk density } \\
\text { TGA, DSC } \\
\text { ICP } \\
\text { IC } \\
\text { Alpha }\end{array}$ \\
\hline
\end{tabular}


Table A-4. Summary of Samples and Analyses. (8 sheets)

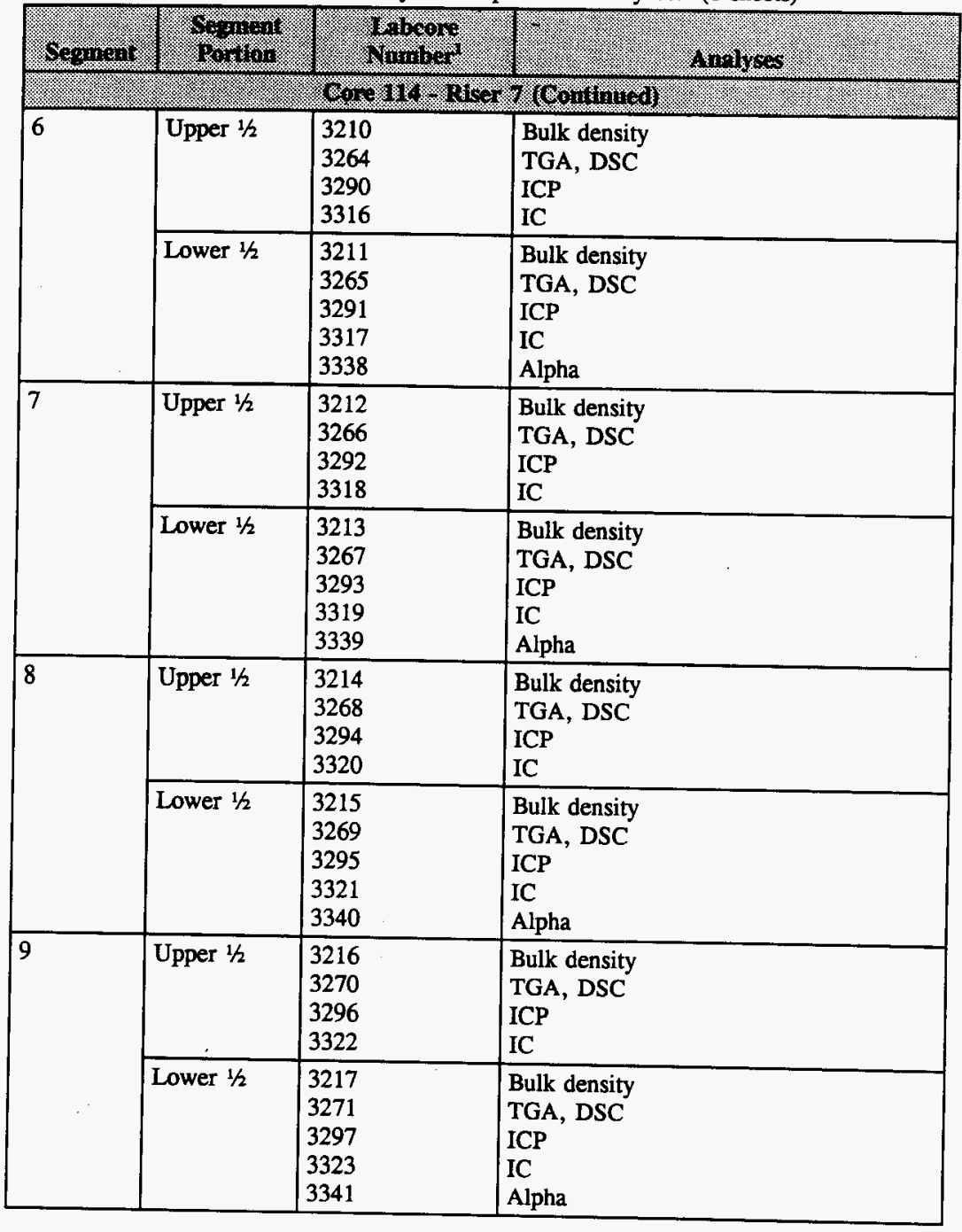


Table A-4. Summary of Samples and Analyses. (8 sheets)

\begin{tabular}{|c|c|c|c|}
\hline 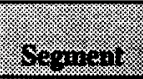 & 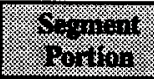 & 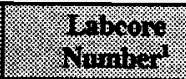 & inilys \\
\hline \multicolumn{4}{|c|}{ 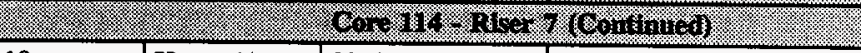 } \\
\hline \multirow[t]{2}{*}{10} & Upper $1 / 2$ & $\begin{array}{l}3218 \\
3272 \\
3298 \\
3324 \\
\end{array}$ & $\begin{array}{l}\text { Bulk density } \\
\text { TGA, DSC } \\
\text { ICP } \\
\text { IC }\end{array}$ \\
\hline & Lower $1 / 2$ & $\begin{array}{l}3219 \\
3273 \\
3299 \\
3325 \\
3342 \\
\end{array}$ & $\begin{array}{l}\text { Bulk density } \\
\text { TGA, DSC } \\
\text { ICP } \\
\text { IC } \\
\text { Alpha } \\
\end{array}$ \\
\hline \multirow[t]{2}{*}{11} & Upper $1 / 2$ & $\begin{array}{l}3220 \\
3274 \\
3300 \\
3326 \\
\end{array}$ & $\begin{array}{l}\text { Bulk density } \\
\text { TGA, DSC } \\
\text { ICP } \\
\text { IC } \\
\end{array}$ \\
\hline & Lower $1 / 2$ & \begin{tabular}{|l|}
3221 \\
3275 \\
3301 \\
3327 \\
3343 \\
\end{tabular} & $\begin{array}{l}\text { Bulk density } \\
\text { TGA, DSC } \\
\text { ICP } \\
\text { IC } \\
\text { Alpha } \\
\end{array}$ \\
\hline \multirow[t]{2}{*}{12} & Upper $1 / 2$ & $\begin{array}{l}3222 \\
3276 \\
3302 \\
3328 \\
\end{array}$ & $\begin{array}{l}\text { Bulk density } \\
\text { TGA, DSC } \\
\text { ICP } \\
\text { IC } \\
\end{array}$ \\
\hline & Lower $1 / 2$ & \begin{tabular}{|l|}
3223 \\
3277 \\
3303 \\
3329 \\
3344 \\
\end{tabular} & $\begin{array}{l}\text { Bulk density } \\
\text { TGA, DSC } \\
\text { ICP } \\
\text { IC } \\
\text { Alpha } \\
\end{array}$ \\
\hline \multirow[t]{2}{*}{13} & Upper $1 / 2$ & $\begin{array}{l}3224 \\
3278 \\
3304 \\
3330\end{array}$ & $\begin{array}{l}\text { Bulk density } \\
\text { TGA, DSC } \\
\text { ICP } \\
\text { IC }\end{array}$ \\
\hline & Lower $1 / 2$ & $\begin{array}{l}3225 \\
3279 \\
3305 \\
3331 \\
3345 \\
\end{array}$ & $\begin{array}{l}\text { Bulk density } \\
\text { TGA, DSC } \\
\text { ICP } \\
\text { IC } \\
\text { Alpha } \\
\end{array}$ \\
\hline
\end{tabular}


Table A-4. Summary of Samples and Analyses. (8 sheets)

\begin{tabular}{|c|c|c|c|}
\hline 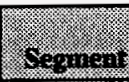 & 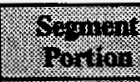 & 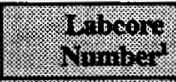 & (1) \\
\hline 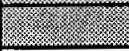 & 3. & \multicolumn{2}{|c|}{ 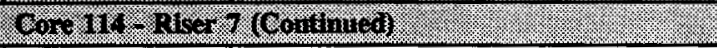 } \\
\hline \multirow[t]{2}{*}{14} & Upper $1 / 2$ & $\begin{array}{l}3226 \\
3280 \\
3306 \\
3332\end{array}$ & $\begin{array}{l}\text { Bulk density } \\
\text { TGA, DSC } \\
\text { ICP } \\
\text { IC }\end{array}$ \\
\hline & Lower $1 / 2$ & $\begin{array}{l}3227 \\
3281 \\
3307 \\
3333 \\
3346 \\
\end{array}$ & $\begin{array}{l}\text { Bulk density } \\
\text { TGA, DSC } \\
\text { ICP } \\
\text { IC } \\
\text { Alpha }\end{array}$ \\
\hline $\mathrm{n} / \mathrm{a}$ & $\mathrm{n} / \mathrm{a}$ & $\begin{array}{l}3349 \\
3770\end{array}$ & $\begin{array}{l}\text { TGA, Specific gravity, DSC, IC, Alpha } \\
\text { ICP }\end{array}$ \\
\hline $\mathrm{n} / \mathrm{a}$ & $\mathrm{n} / \mathrm{a}$ & 2952 & ICP, IC \\
\hline
\end{tabular}

Notes:

$\mathbf{n} / \mathbf{a}=$ not applicable

'All laboratory sample numbers begin with $\$ 95 T 00$. 
WHC-SD-WM-ER-581 Rev. 0

Table A-5. Analytical Procedures.

\begin{tabular}{|c|c|c|c|}
\hline 1.10 & 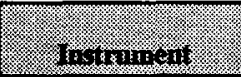 & 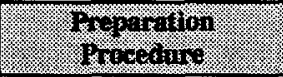 & 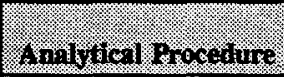 \\
\hline Energetics by DSC & $\begin{array}{l}\text { Mettler } \\
\text { Perkin-Elmer }\end{array}$ & $n / a$ & $\begin{array}{l}\text { LA-514-113, Rev. C-1 } \\
\text { LA-514-114, Rev. C-1 }\end{array}$ \\
\hline $\begin{array}{l}\text { Percent water by } \\
\text { TGA }\end{array}$ & $\begin{array}{l}\text { Mettler } \\
\text { Perkin-Elmer } \\
\text { TM }\end{array}$ & $\mathrm{n} / \mathrm{a}$ & $\begin{array}{l}\text { LA-560-112, Rev. B-1 } \\
\text { LA-514-114, Rev. C-1 }\end{array}$ \\
\hline Total alpha activity & $\begin{array}{l}\text { Alpha proportional } \\
\text { counter }\end{array}$ & LA-549-141, Rev. D-0 & LA-508-101, Rev. D-2 \\
\hline Solid bulk density & $\mathrm{n} / \mathrm{a}$ & $n / a$ & LO-160-103, Rev. A-7 \\
\hline $\begin{array}{l}\text { Liquid specific } \\
\text { gravity }\end{array}$ & $\mathrm{n} / \mathrm{a}$ & $n / a$ & LA-510-112, Rev. C-3 \\
\hline $\begin{array}{l}\text { Lithium and other } \\
\text { metals by ICP }\end{array}$ & $\begin{array}{l}\text { Inductively coupled } \\
\text { plasma } \\
\text { spectrometer }\end{array}$ & LA-505-159, Rev. C-0 & $\begin{array}{l}\text { LA-505-151, Rev. D-3 } \\
\text { LA-505-161, Rev. B-0 }\end{array}$ \\
\hline $\begin{array}{l}\text { Bromide and other } \\
\text { anions by IC }\end{array}$ & Ion chromatograph & LA-504-101, Rev. D-0 & LA-533-105, Rev. D-1 \\
\hline $\begin{array}{l}\text { Radionuclides by } \\
\text { GEA }\end{array}$ & $\begin{array}{l}\text { Gamma detector } \\
\text { spectrometer }\end{array}$ & LA-549-141, Rev. D-0 & LA-548-121, Rev. D-1 \\
\hline $\begin{array}{l}{ }^{90} \mathrm{Sr} \text { by separation } \\
\text { and beta counting }\end{array}$ & $\begin{array}{l}\text { Beta proportional } \\
\text { counter }\end{array}$ & LA-549-141, Rev. D-0 & LA-220-101, Rev. D-1 \\
\hline
\end{tabular}

Notes:

$\mathbf{n} / \mathbf{a}=$ not applicable

Rev. = revision 
WHC-SD-WM-ER-581 Rev. 0

This page intentionally left blank. 
WHC-SD-WM-ER-581 Rev. 0

APPENDIX B

ANALYTICAL RESULTS FROM 1995 CORE SAMPLING 
WHC-SD-WM-ER-581 Rev. 0

This page intentionally left blank. 


\section{APPENDDX B}

\section{ANALYTICAL RESULTS FROM 1995 CORE SAMPLING}

\section{B.1 INTRODUCTION}

Appendix B reports the chemical, radiochemical, and physical characteristics of tank 241-B-204 in table form and in terms of the specific concentrations of metals, ions, radionuclides, and physical properties.

Tables B-1 through B-53 list the following information: laboratory sample identification; sample origin (core/segment/subsegment); an original and duplicate result for each sample; a sample mean; a mean for the tank in which all cores, segments, and subsegments are weighted equally; a relative standard deviation of the mean (RSD [Mean]); and a projected tank inventory for the particular analyte using the weighted mean and the appropriate conversion factors. The projected tank inventory column is not applicable to the percent water, DSC, TGA, or bulk density data. The data are listed in standard notation for values greater than 0.001 and less than 100,000. Values outside these limits are listed in scientific notation.

A description of the units and symbols used in the analyte tables and the sources used in compiling the analytical data (Sasaki 1996b) are found in the List of Terms and Section 7.0, respectively. For information on sampling rationale, locations, and descriptions of sampling events, see Section 3.0 and Appendix A.

\section{B.2 ANALYTE TABLE DESCRIPTION}

Tables B-1 through B-50 describe the organization of the chemical species and radionuclide tables. Tables B-51 through B-53 contain the TGA, DSC, and density results; the organization of these tables is similar to the chemical species tables with only slight variations.

Column 1 lists the laboratory sample for which the analyte was measured.

Column 2 lists the core and segment from which each sample was derived. The first number is the core number; it is followed by a colon and the segment number.

Column 3 lists the name of the segment portion (subsegment) from which the sample was taken. This can be the entire segment (whole), the drainable liquid portion (DL), or the upper or lower half segment portions. 
Columns 4 and 5 (Result and Duplicate) are self-explanatory. The "Mean" is the average of the result and duplicate values. All values, including those below the detection level (indicated by the less-than symbol, <), were averaged in calculating the sample means. If the result and duplicate values were both nondetected, the mean is expressed as a nondetected value. If one of the two values is nondetected and one is detected, or if both are detected, then the sample mean is reported as a detected value. The result and duplicate values and the result/duplicate means are reported in the tables exactly as found in the original laboratory data package. The means may appear to have been rounded up in some cases and rounded down in others. This is because the analytical results given in the tables may have fewer significant figures than originally reported not because the means were incorrectly calculated.

Column 7 is the overall (or analyte concentration) means for the waste in tank 241-B-204; they were calculated as follows:

The drainable liquid means were calculated by averaging the segment means within a core, then averaging the two core means to obtain an overall mean.

The estimated overall sludge mean based on segment level data were calculated in the following manner. The sample/duplicate results within a subsegment were averaged to obtain a subsegment mean, subsegment means were averaged to obtain a segment mean, then segment means were averaged to obtain a core mean, and finally the two core means were averaged to obtain the overall mean.

Column 8 (RSD [Mean]) is 100 times the standard deviation of the mean divided by the overall tank mean. The standard deviation of the mean was estimated using standard ANOVA statistical techniques. Relative standard deviations of the mean were not computed for analytes that had greater than 50 percent nondetected values. For analytes with 50 percent or more detected results, all data for a given analyte were used in the calculation.

Column 9 (projected inventory) is the product of the overall analyte concentration mean, the volume of tank waste ( $185 \mathrm{~kL}$ for the sludge and $4 \mathrm{~kL}$ for the drainable liquid), the bulk density of the waste $(1.19 \mathrm{~g} / \mathrm{mL}$ for the sludge and $1.05 \mathrm{~g} / \mathrm{mL}$ for the drainable liquid), and the appropriate conversion factors.

The four quality control parameters assessed on the tank 241-B-204 samples were standard recoveries, spike recoveries, duplicate analyses (RPDs), and blanks. These were summarized in Section 5.1.2. Sample and duplicate pairs, in which any quality control parameters were outside their specified limits, are footnoted in column 6 of the following tables. Limits for quality control parameters were specified only for total alpha, TGA, DSC, specific gravity, bulk density, lithium, and bromide analyses because these were the analyses required by the applicable DQO. 
Table B-1. Tank 241-B-204 Analytical Results: Aluminum. (3 sheets)

\begin{tabular}{|c|c|c|c|c|c|c|c|c|}
\hline Singor & 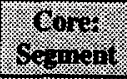 & ocion & Ifosing & Higlinate & 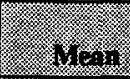 & $x_{304}$ & $\frac{1963 \%}{(0.39}$ & 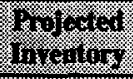 \\
\hline Noritu & & X:- & 89 & 18 & 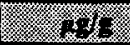 & 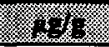 & 3 & $\frac{18}{85}$ \\
\hline S95T002980 & $112: 2$ & Whole & 448.0 & 397.0 & 422.5 & \multirow[t]{21}{*}{65.3} & \multirow[t]{21}{*}{36.5} & \multirow[t]{21}{*}{14.4} \\
\hline S95T003061 & \multirow[t]{2}{*}{$112: 3$} & Upper $1 / 2$ & 64.10 & 79.5 & 71.8 & & & \\
\hline \$95T003062 & & Lower $1 / 2$ & 55.90 & 53.20 & 54.55 & & & \\
\hline \$95T003063 & \multirow[t]{2}{*}{$112: 4$} & Upper $1 / 2$ & 59.00 & 74.70 & 66.85 & & & \\
\hline S95T003064 & & Lower $1 / 2$ & 48.80 & 49.40 & 49.10 & & & \\
\hline \$95T003065 & \multirow[t]{2}{*}{$112: 5$} & Upper $1 / 2$ & 57.70 & 45.00 & 51.35 & & & \\
\hline S95T003066 & & Lower $1 / 2$ & 66.20 & 60.70 & 63.45 & & & \\
\hline S95T003067 & \multirow[t]{2}{*}{$112: 6$} & Upper $1 / 2$ & 63.00 & 71.60 & 67.30 & & & \\
\hline S95T003068 & & Lower $1 / 2$ & 56.50 & 51.40 & 53.95 & & & \\
\hline S95T003069 & \multirow[t]{2}{*}{$112: 7$} & Upper $1 / 2$ & 65.60 & 69.90 & 67.75 & & & \\
\hline S95T003070 & & Lower $1 / 2$ & 64.40 & 49.50 & 56.95 & & & \\
\hline S95T003071 & \multirow[t]{2}{*}{$112: 8$} & Upper $1 / 2$ & 44.70 & 43.10 & 43.90 & & & \\
\hline S95T003072 & & Lower $1 / 2$ & 45.70 & 53.60 & 49.65 & & & \\
\hline S95T003073 & \multirow[t]{2}{*}{$112: 9$} & Upper $1 / 2$ & 51.90 & 54.60 & 53.25 & & & \\
\hline S95T003074 & & Lower $1 / 2$ & 324.0 & 72.60 & 198.3 & & & \\
\hline S95T003075 & \multirow[t]{2}{*}{$112: 10$} & Upper $1 / 2$ & 47.90 & 59.10 & 53.50 & & & \\
\hline S95T003076 & & Lower $1 / 2$ & 50.30 & 46.00 & 48.15 & & & \\
\hline S95T003077 & \multirow[t]{2}{*}{$112: 11$} & Upper $1 / 2$ & 47.50 & 46.10 & 46.80 & & & \\
\hline S95T003078 & & Lower $1 / 2$ & $<47.4$ & $<57.4$ & $<52.4$ & & & \\
\hline 6S95T003079 & \multirow[t]{2}{*}{ 112:12 } & Upper $1 / 2$ & $<53.5$ & $<48.3$ & $<50.9$ & & & \\
\hline S95T003080 & & Lower $1 / 2$ & 52.90 & $<50.4$ & 51.7 & & & \\
\hline
\end{tabular}


Table B-1. Tank 241-B-204 Analytical Results: Aluminum. (3 sheets)

\begin{tabular}{|c|c|c|c|c|c|c|c|c|}
\hline 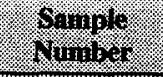 & 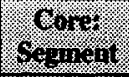 & $\begin{array}{l}0.13 \\
0.0110 \% 11\end{array}$ & Hovili & 1.310.10 & Sena & $6 \%$ & 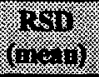 & 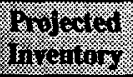 \\
\hline $8014 \mathrm{~s}$ & & & 1910 & 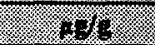 & S:- & $.1 \%$ & (1) & $4 y$ \\
\hline S95T003081 & \multirow[t]{2}{*}{$112: 13$} & Upper $1 / 2$ & $<43.6$ & 44.70 & 44.15 & & & \\
\hline S95T003082 & & Lower $1 / 2$ & $<48.6$ & $<42.4$ & $<45.5$ & & & \\
\hline S95T003083 & \multirow[t]{2}{*}{$112: 14$} & Upper $1 / 2$ & 46.30 & $<52.9$ & 49.6 & & & \\
\hline S95T003084 & & Lower $1 / 2$ & $<99.6$ & $<90.5$ & $<95.1$ & & & \\
\hline S95T003282 & \multirow[t]{2}{*}{$114: 2$} & Upper $1 / 2$ & 63.90 & 60.30 & 62.10 & & & \\
\hline S95T003283 & & Lower $1 / 2$ & 19.30 & 16.50 & 17.90 & & & \\
\hline S95T003284 & \multirow[t]{2}{*}{$114: 3$} & Upper $1 / 2$ & 26.40 & 19.80 & 23.10 & & & \\
\hline S95T003285 & & Lower $1 / 2$ & 93.40 & $<96.6$ & 95 & & & \\
\hline S95T003286 & \multirow[t]{2}{*}{$114: 4$} & Upper $1 / 2$ & 26.50 & 25.60 & 26.05 & & & \\
\hline S95T003287 & & Lower $1 / 2$ & 48.10 & 35.10 & 41.60 & & & \\
\hline \$95T003288 & \multirow[t]{2}{*}{$114: 5$} & Upper $1 / 2$ & 30.90 & 34.40 & 32.65 & & & \\
\hline S95T003289 & & Lower $1 / 2$ & 42.30 & 33.30 & 37.80 & & & \\
\hline S95T003290 & \multirow[t]{2}{*}{$114: 6$} & Upper $1 / 2$ & 35.70 & 43.70 & 39.70 & & & \\
\hline S95T003291 & & Lower $1 / 2$ & 28.20 & 33.10 & 30.65 & & & \\
\hline S95T003292 & \multirow[t]{2}{*}{$114: 7$} & Upper $1 / 2$ & 23.20 & 32.90 & 28.05 & & & \\
\hline S95T003293 & & Lower $1 / 2$ & 48.00 & 28.70 & 38.35 & & & \\
\hline S95T003294 & \multirow[t]{2}{*}{$114: 8$} & Upper $1 / 2$ & 62.10 & 49.20 & 55.65 & & & \\
\hline S95T003295 & & Lower $1 / 2$ & 21.30 & 35.50 & 28.40 & & & \\
\hline S95T003296 & \multirow[t]{2}{*}{$114: 9$} & Upper $1 / 2$ & 41.10 & 44.80 & 42.95 & & & \\
\hline S95T003297 & & Lower $1 / 2$ & 41.30 & 37.80 & 39.55 & & & \\
\hline
\end{tabular}


Table B-1. Tank 241-B-204 Analytical Results: Aluminum. (3 sheets)

\begin{tabular}{|c|c|c|c|c|c|c|c|c|}
\hline $\begin{array}{l}\text { Ganple } \\
\text { yonimser }\end{array}$ & 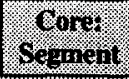 & 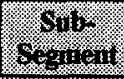 & (19uili: & bipitalo & tenis: & $6 \%$ & 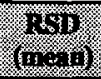 & 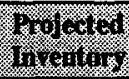 \\
\hline Solus & (n) & (4) & $18 / \mathrm{g}$ & 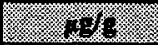 & .98 & 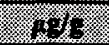 & 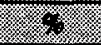 & 36 \\
\hline \$95T003298 & \multirow[t]{2}{*}{$114: 10$} & Upper $1 / 2$ & 37.50 & 28.00 & 32.75 & & & \\
\hline$\$ 951003299$ & & Lower $1 / 2$ & 40.00 & 59.90 & 49.95 & & & \\
\hline S95T003300 & \multirow[t]{2}{*}{$114: 11$} & Upper $1 / 2$ & 46.30 & 43.20 & 44.75 & & & \\
\hline S95T003301 & & Lower $1 / 2$ & 37.20 & 37.40 & 37.30 & & & \\
\hline \$95T003302 & \multirow[t]{2}{*}{$114: 12$} & Upper $1 / 2$ & 35.10 & 42.50 & 38.80 & & & \\
\hline S95T003303 & & Lower $1 / 2$ & 37.10 & 39.30 & 38.20 & & & \\
\hline S95T003304 & \multirow[t]{2}{*}{$114: 13$} & Upper $1 / 2$ & 44.80 & 39.50 & 42.15 & & & \\
\hline S95T003305 & & Lower $1 / 2$ & 44.50 & 43.00 & 43.75 & & & \\
\hline S95T003306 & \multirow[t]{2}{*}{$114: 14$} & Upper $1 / 2$ & 46.40 & 48.80 & 47.60 & & & \\
\hline S95T003307 & & Lower $1 / 2$ & 43.60 & 53.10 & 48.35 & & & \\
\hline \multicolumn{3}{|c|}{ 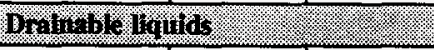 } & $(1,8111$ & (3) & $13 / \ln$ & \% & (2) & (2) \\
\hline S95T002976 & 112:1 & DL & $<10.0$ & $<10.0$ & $<10.0$ & \multirow[t]{3}{*}{$<7.53$} & \multirow[t]{3}{*}{$n / a$} & \multirow[t]{3}{*}{$<0.0301$} \\
\hline S95T002984 & $112: 2$ & DL & $<10.0$ & $<10.0$ & $<10.0$ & & & \\
\hline S95T003255 & $114: 1$ & $\mathrm{DL}$ & $<5.05$ & $<5.05$ & $<5.05$ & & & \\
\hline
\end{tabular}


Table B-2. Tank 241-B-204 Analytical Results: Antimony. (3 sheets)

\begin{tabular}{|c|c|c|c|c|c|c|c|c|}
\hline 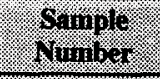 & wor & $x_{0}^{4}$ & in aning & Diplite & 40.11 & 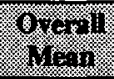 & 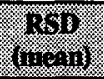 & 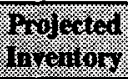 \\
\hline 8.11) & স & ; & . & (n) & 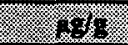 & ing & 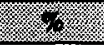 & $8 \%$ \\
\hline S95T002980 & $112: 2$ & Whole & $<16.6$ & $<22.3$ & $<19.5$ & \multirow[t]{21}{*}{$<26.9$} & \multirow[t]{21}{*}{$\mathrm{n} / \mathrm{a}$} & \multirow[t]{21}{*}{$<5.92$} \\
\hline S95T003061 & \multirow[t]{2}{*}{$112: 3$} & Upper $1 / 2$ & $<14.6$ & $<11.3$ & $<13.0$ & & & \\
\hline S95T003062 & & Lower $1 / 2$ & $<13.5$ & $<12.8$ & $<13.2$ & & & \\
\hline S95T003063 & \multirow[t]{2}{*}{$112: 4$} & Upper $1 / 2$ & $<10.5$ & $<14.0$ & $<12.3$ & & & \\
\hline \$95T003064 & & Lower $1 / 2$ & $<14.6$ & $<13.3$ & $<14.0$ & & & \\
\hline S95T003065 & \multirow[t]{2}{*}{$112: 5$} & Upper $1 / 2$ & $<26.2$ & $<24.9$ & $<25.6$ & & & \\
\hline S95T003066 & & Lower $1 / 2$ & $<13.8$ & $<11.7$ & $<12.8$ & & & \\
\hline S95T003067 & \multirow[t]{2}{*}{$112: 6$} & Upper 1/2 & $<27.9$ & $<20.7$ & $<24.3$ & & & \\
\hline S95T003068 & & Lower $1 / 2$ & $<13.4$ & $<11.6$ & $<12.5$ & & & \\
\hline S95T003069 & \multirow[t]{2}{*}{$112: 7$} & Upper $1 / 2$ & $<22.0$ & $<22.8$ & $<22.4$ & & & \\
\hline S95T003070 & & Lower $1 / 2$ & $<21.1$ & $<20.6$ & $<20.9$ & & & \\
\hline S95T003071 & \multirow[t]{2}{*}{$112: 8$} & Upper $1 / 2$ & $<12.6$ & $<13.8$ & $<13.2$ & & & \\
\hline S95T003072 & & Lower $1 / 2$ & $<23.4$ & $<22.9$ & $<23.2$ & & & \\
\hline S95T003073 & \multirow[t]{2}{*}{$112: 9$} & Upper $1 / 2$ & $<24.8$ & $<22.8$ & $<23.8$ & & & \\
\hline \$95T003074 & & Lower $1 / 2$ & $<20.7$ & $<22.9$ & $<21.8$ & & & \\
\hline S95T003075 & \multirow[t]{2}{*}{ 112: 10} & Upper $1 / 2$ & $<22.3$ & $<21.8$ & $<22.1$ & & & \\
\hline S95T003076 & & Lower $1 / 2$ & $<27.4$ & $<24.6$ & $<26.0$ & & & \\
\hline S95T003077 & \multirow[t]{2}{*}{ 112:11 } & Upper $1 / 2$ & $<24.4$ & $<20.9$ & $<22.7$ & & & \\
\hline S95T003078 & & Lower $1 / 2$ & $<56.9$ & $<68.8$ & $<62.9$ & & & \\
\hline S95T003079 & \multirow[t]{2}{*}{$112: 12$} & Upper $1 / 2$ & $<64.3$ & $<57.9$ & $<61.1$ & & & \\
\hline S95T003080 & & Lower $1 / 2$ & $<58.7$ & $<60.5$ & $<59.6$ & & & \\
\hline
\end{tabular}




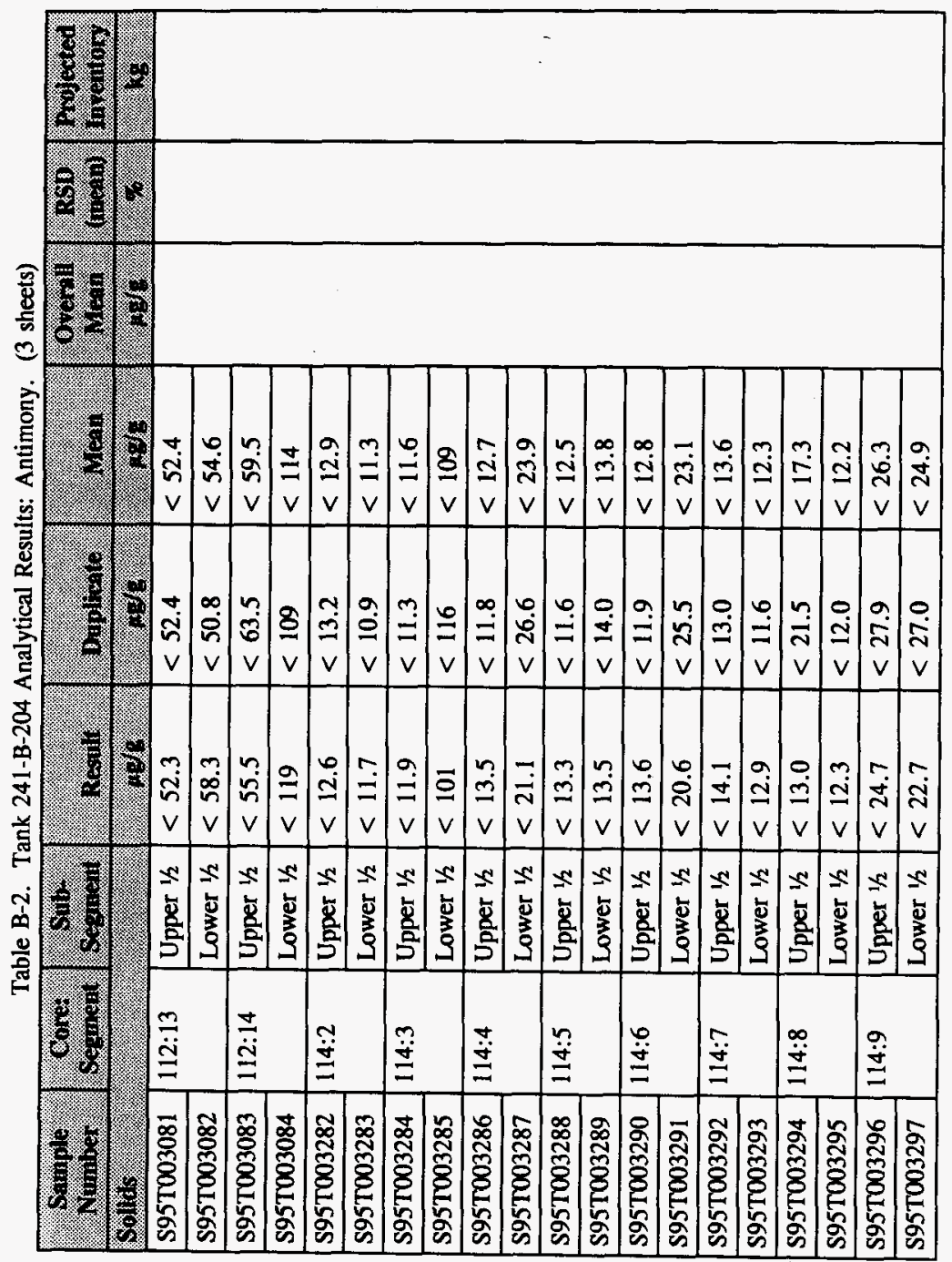


Table B-2. Tank 241-B-204 Analytical Results: Antimony. (3 sheets)

\begin{tabular}{|c|c|c|c|c|c|c|c|c|}
\hline $\begin{array}{l}\text { Oningte } \\
\text { Vuminger }\end{array}$ & (6) & 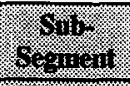 & Inosin! & 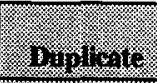 & (3) & \% & $\frac{6193}{(10 \%)}$ & 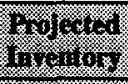 \\
\hline 5013 & & & (1) & \% & .830 & 20 & ( & (t) \\
\hline S95T003298 & \multirow[t]{2}{*}{ 114: 10} & Upper $1 / 2$ & $<22.2$ & $<21.5$ & $<21.9$ & & & \\
\hline \$95T003299 & & Lower $1 / 2$ & $<20.3$ & $<23.1$ & $<21.7$ & & & \\
\hline \$95T003300 & \multirow[t]{2}{*}{$114: 11$} & Upper $1 / 2$ & $<28.2$ & $<29.0$ & $<28.6$ & & & \\
\hline S95T003301 & & Lower $1 / 2$ & $<20.4$ & $<20.4$ & $<20.4$ & & & \\
\hline \$95T003302 & \multirow[t]{2}{*}{$114: 12$} & Upper $1 / 2$ & $<14.4$ & $<13.4$ & $<13.9$ & & & \\
\hline S95T003303 & & Lower $1 / 2$ & $<13.4$ & $<22.2$ & $<17.8$ & & & \\
\hline \$95T003304 & \multirow[t]{2}{*}{$114: 13$} & Upper $1 / 2$ & $<27.5$ & $<24.5$ & $<26.0$ & & & \\
\hline S95T003305 & & Lower $1 / 2$ & $<21.6$ & $<21.9$ & $<21.8$ & & & \\
\hline S95T003306 & \multirow[t]{2}{*}{ 114:14 } & Upper $1 / 2$ & $<25.9$ & $<25.6$ & $<25.8$ & & & \\
\hline S95T003307 & & Lower $1 / 2$ & $<23.2$ & $<25.3$ & $<24.3$ & & & \\
\hline \multicolumn{3}{|c|}{ 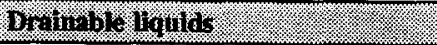 } & 4.11日1) & \% & . & 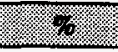 & (2) & 164 \\
\hline S95T002976 & 112:1 & DL & $<12.1$ & $<12.1$ & $<12.1$ & \multirow[t]{3}{*}{$<9.08$} & \multirow[t]{3}{*}{$n / a$} & \multirow[t]{3}{*}{$<0.0363$} \\
\hline S95T002984 & $112: 2$ & DL & $<12.1$ & $<12.1$ & $<12.1$ & & & \\
\hline S95T003255 & 114:1 & DL & $<6.06$ & $<6.06$ & $<6.06$ & & & \\
\hline
\end{tabular}


Table B-3. Tank 241-B-204 Analytical Results: Arsenic. (3 sheets)

\begin{tabular}{|c|c|c|c|c|c|c|c|c|}
\hline 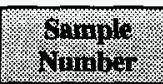 & 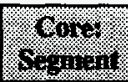 & ofing & 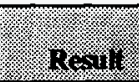 & $3,130 \%$ & 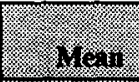 & 9\%,nin & 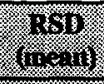 & 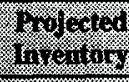 \\
\hline Sollos & & & . & 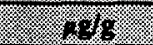 & 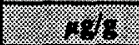 &.$\%$ & (1) & (2) \\
\hline S95T002980 & $112: 2$ & Whole & $<27.6$ & $<37.1$ & $<32.35$ & \multirow[t]{21}{*}{$<44.8$} & \multirow[t]{21}{*}{$n / a$} & \multirow[t]{21}{*}{$<9.86$} \\
\hline S95T003061 & \multirow[t]{2}{*}{$112: 3$} & Upper $1 / 2$ & $<24.1$ & $<18.8$ & $<21.5$ & & & \\
\hline S95T003062 & & Lower $1 / 2$ & $<22.5$ & $<21.3$ & $<21.9$ & & & \\
\hline S95T003063 & \multirow[t]{2}{*}{$112: 4$} & Upper $1 / 2$ & $<17.6$ & $<23.3$ & $<20.5$ & & & \\
\hline \$95T003064 & & Lower $1 / 2$ & $<24.3$ & $<22.2$ & $<23.3$ & & & \\
\hline S95T003065 & \multirow[t]{2}{*}{$112: 5$} & Upper $1 / 2$ & $<43.7$ & $<41.6$ & $<42.7$ & & & \\
\hline \$95T003066 & & Lower $1 / 2$ & $<23.1$ & $<19.5$ & $<21.3$ & & & \\
\hline \$95T003067 & \multirow[t]{2}{*}{$112: 6$} & Upper $1 / 2$ & $<46.6$ & $<34.6$ & $<40.6$ & & & \\
\hline S95T003068 & & Lower $1 / 2$ & $<22.3$ & $<19.4$ & $<20.9$ & & & \\
\hline \$95T003069 & \multirow[t]{2}{*}{$112: 7$} & Upper $1 / 2$ & $<36.4$ & $<38.1$ & $<37.3$ & & & \\
\hline S95T003070 & & Lower $1 / 2$ & $<35.1$ & $<34.3$ & $<34.7$ & & & \\
\hline S95T003071 & \multirow[t]{2}{*}{$112: 8$} & Upper $1 / 2$ & $<21.0$ & $<23.0$ & $<22.0$ & & & \\
\hline S95T003072 & & Lower $1 / 2$ & $<39.1$ & $<38.1$ & $<38.6$ & & & \\
\hline S95T003073 & \multirow[t]{2}{*}{$112: 9$} & Upper $1 / 2$ & $<41.4$ & $<38.0$ & $<39.7$ & & & \\
\hline S95T003074 & & Lower $1 / 2$ & $<34.3$ & $<38.1$ & $<36.2$ & & & \\
\hline S95T003075 & \multirow[t]{2}{*}{$112: 10$} & Upper $1 / 2$ & $<37.2$ & $<36.4$ & $<36.8$ & & & \\
\hline S95T003076 & & Lower $1 / 2$ & $<45.6$ & $<41.1$ & $<43.4$ & & & \\
\hline \$95T003077 & \multirow[t]{2}{*}{$112: 11$} & Upper $1 / 2$ & $<40.7$ & $<34.8$ & $<37.8$ & & & \\
\hline S95T003078 & & Lower $1 / 2$ & $<94.9$ & $<115$ & $<105$ & & & \\
\hline S95T003079 & \multirow[t]{2}{*}{$112: 12$} & Upper $1 / 2$ & $<107$ & $<96.6$ & $<102$ & & & \\
\hline S95T003080 & & Lower $1 / 2$ & $<97.8$ & $<101$ & $<99.4$ & & & \\
\hline
\end{tabular}


Table B-3. Tank 241-B-204 Analytical Results: Arsenic. (3 sheets)

\begin{tabular}{|c|c|c|c|c|c|c|c|c|}
\hline Shinplo & 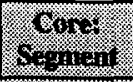 & 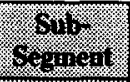 & ingul & 13 & 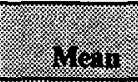 & 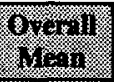 & $\frac{1}{4.13}$ & 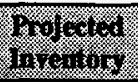 \\
\hline (3)10\% & 2. & (T) & ritis & . & (1) & 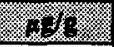 & 4 & 36 \\
\hline S95T003081 & \multirow[t]{2}{*}{$112: 13$} & Upper 1/2 & $<87.2$ & $<87.3$ & $<87.3$ & & & \\
\hline$\$ 95 T 003082$ & & Lower $1 / 2$ & $<97.2$ & $<84.7$ & $<91.0$ & & & \\
\hline S95T003083 & \multirow[t]{2}{*}{$112: 14$} & Upper $1 / 2$ & $<92.6$ & $<106$ & $<99.3$ & & & \\
\hline S95T003084 & & Lower $1 / 2$ & $<199$ & $<181$ & $<190$ & & & \\
\hline S95T003282 & \multirow[t]{2}{*}{$114: 2$} & Upper $1 / 2$ & $<21.0$ & $<22.0$ & $<21.5$ & & & \\
\hline S95T003283 & & Lower $1 / 2$ & $<19.5$ & $<18.2$ & $<18.9$ & & & \\
\hline S95T003284 & \multirow[t]{2}{*}{$114: 3$} & Upper $1 / 2$ & $<19.8$ & $<18.9$ & $<19.4$ & & & \\
\hline S95T003285 & & Lower $1 / 2$ & $<169$ & $<193$ & $<181$ & & & \\
\hline S95T003286 & \multirow[t]{2}{*}{$114: 4$} & Upper $1 / 2$ & $<22.3$ & $<19.7$ & $<21.0$ & & & \\
\hline S95T003287 & & Lower $1 / 2$ & $<35.1$ & $<44.3$ & $<39.7$ & & & \\
\hline S95T003288 & \multirow[t]{2}{*}{$114: 5$} & Upper $1 / 2$ & $<22.2$ & $<19.3$ & $<20.8$ & & & \\
\hline \$95T003289 & & Lower $1 / 2$ & $<22.6$ & $<23.4$ & $<23.0$ & & & \\
\hline S95T003290 & \multirow[t]{2}{*}{$114: 6$} & Upper $1 / 2$ & $<22.6$ & $<19.8$ & $<21.2$ & & & \\
\hline S95T003291 & & Lower $1 / 2$ & $<34.4$ & $<42.5$ & $<38.5$ & & & \\
\hline S95T003292 & \multirow[t]{2}{*}{$114: 7$} & Upper $1 / 2$ & $<23.4$ & $<21.7$ & $<22.6$ & & & \\
\hline S95T003293 & & Lower $1 / 2$ & $<21.6$ & $<19.4$ & $<20.5$ & & & \\
\hline S95T003294 & \multirow[t]{2}{*}{$114: 8$} & Upper $1 / 2$ & $<21.7$ & $<35.8$ & $<28.8$ & & & \\
\hline S95T003295 & & Lower $1 / 2$ & $<20.5$ & $<20.0$ & $<20.3$ & & & \\
\hline S95T003296 & \multirow[t]{2}{*}{$114: 9$} & Upper $1 / 2$ & $<41.1$ & $<46.4$ & $<43.8$ & & & \\
\hline S95T003297 & & Lower $1 / 2$ & $<37.9$ & $<45.0$ & $<41.5$ & & & \\
\hline
\end{tabular}


Table B-3. Tank 241-B-204 Analytical Results: Arsenic. (3 sheets)

\begin{tabular}{|c|c|c|c|c|c|c|c|c|}
\hline Oanple & 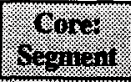 & 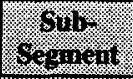 & 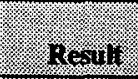 & (3) & iner. & 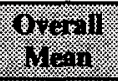 & $\frac{1}{403}$ & 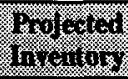 \\
\hline Allias & (2) & (3. & (3. & 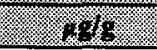 & , 15 & (19/6) & , & ; \\
\hline S95T003298 & \multirow[t]{2}{*}{$114: 10$} & Upper $1 / 2$ & $<37.0$ & $<35.9$ & $<36.5$ & & & \\
\hline S95T003299 & & Lower $1 / 2$ & $<33.9$ & $<38.5$ & $<36.2$ & & & \\
\hline S95T003300 & \multirow[t]{2}{*}{$114: 11$} & Upper $1 / 2$ & $<47.0$ & $<48.3$ & $<47.7$ & & & \\
\hline S95T003301 & & Lower $1 / 2$ & $<34.0$ & $<34.0$ & $<34.0$ & & & \\
\hline S95T003302 & \multirow[t]{2}{*}{$114: 12$} & Upper $1 / 2$ & $<24.0$ & $<22.1$ & $<23.1$ & & & \\
\hline S95T003303 & & Lower $1 / 2$ & $<22.3$ & $<37.0$ & $<29.7$ & & & \\
\hline S95T003304 & \multirow[t]{2}{*}{$114: 13$} & Upper $1 / 2$ & $<45.8$ & $<40.9$ & $<.43 .4$ & & & \\
\hline S95T003305 & & Lower $1 / 2$ & $<36.0$ & $<\overline{36.6}$ & $<36.3$ & & & \\
\hline S95T003306 & \multirow[t]{2}{*}{ 114:14 } & Upper $1 / 2$ & $<42.9$ & $<42.7$ & $<42.8$ & & & \\
\hline \$95T003307 & & Lower $1 / 2$ & $<38.6$ & $<42.2$ & $<40.4$ & & & \\
\hline \multicolumn{3}{|c|}{ Dininable fiquits } & Hojm & iginu & a 1311 & W) & (1) & 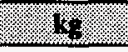 \\
\hline S95T002976 & 112:1 & DL & $<20.1$ & $<20.1$ & $<20.1$ & \multirow[t]{3}{*}{$<15.1$} & \multirow[t]{3}{*}{$n / a$} & \multirow[t]{3}{*}{$<0.0604$} \\
\hline S95T002984 & $112: 2$ & $\mathrm{DL}$ & $<20.1$ & $<20.1$ & $<20.1$ & & & \\
\hline \$95T003255 & $114: 1$ & $\mathrm{DL}$ & $<10.1$ & $<10.1$ & $<10.1$ & & & \\
\hline
\end{tabular}




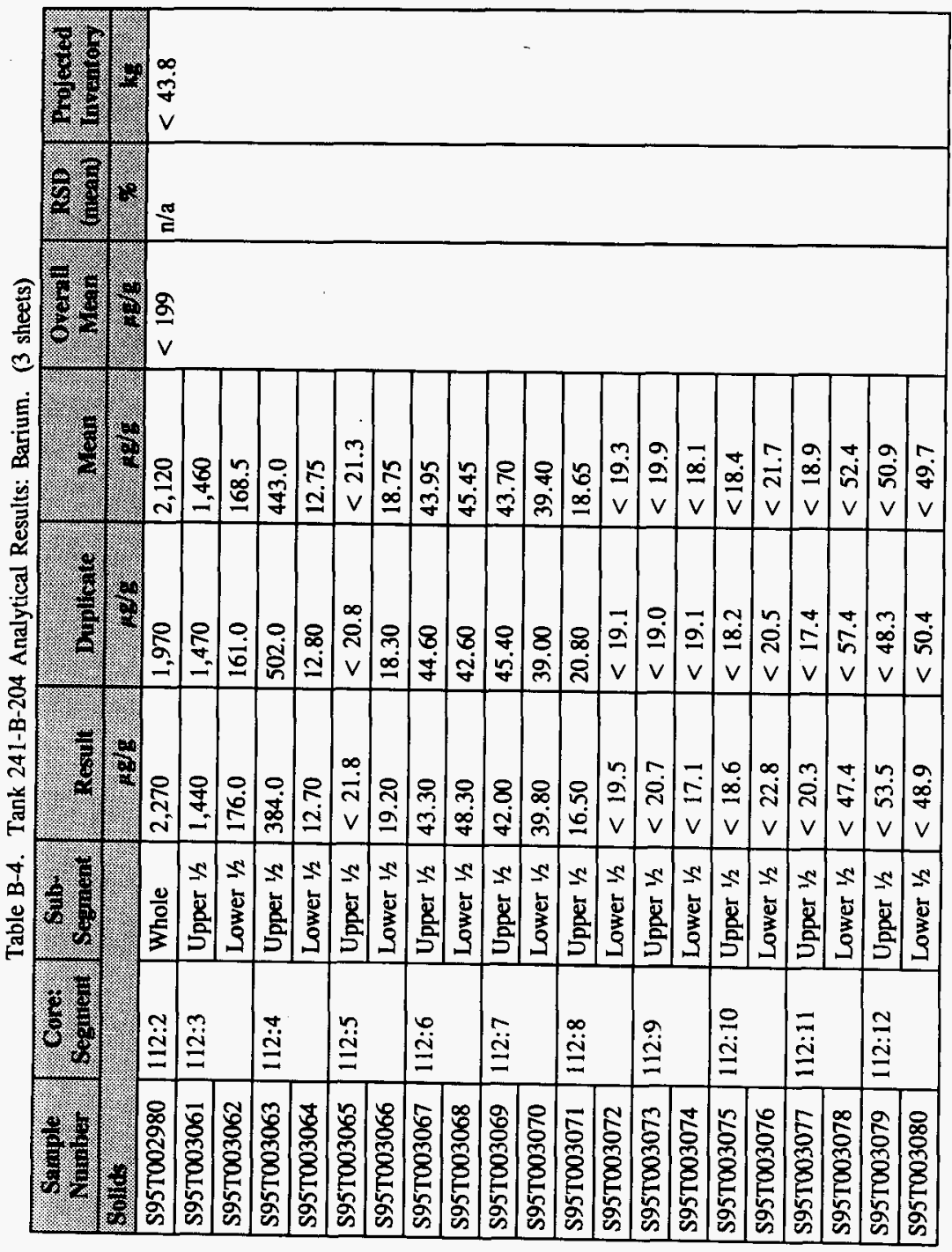


Table B-4. Tank 241-B-204 Analytical Results: Barium. (3 sheets)

\begin{tabular}{|c|c|c|c|c|c|c|c|c|}
\hline sompl & agror & $64 \cos 11$ & \% & Bupis:or. & (1) & 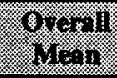 & 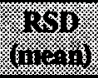 & 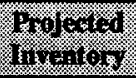 \\
\hline 6019 & & (1) & $(1 /)^{2}$ & 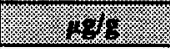 & 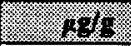 & s. & 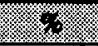 & 18 \\
\hline S95T003081 & \multirow[t]{2}{*}{$112: 13$} & Upper $1 / 2$ & $<43.6$ & $<43.6$ & $<43.6$ & & & \multirow{20}{*}{1} \\
\hline S95T003082 & & Lower $1 / 2$ & $<48.6$ & $<42.4$ & $<45.5$ & & & \\
\hline S95T003083 & \multirow[t]{2}{*}{$112: 14$} & Upper $1 / 2$ & $<46.3$ & $<52.9$ & $<49.6$ & & & \\
\hline \$95T003084 & & Lower $1 / 2$ & $<99.6$ & $<90.5$ & $<95.1$ & & & \\
\hline S95T003282 & \multirow[t]{2}{*}{$114: 2$} & Upper $1 / 2$ & 2,020 & 1,990 & 2,005 & & & \\
\hline \$95T003283 & & Lower $1 / 2$ & 253.0 & 261.0 & 257.0 & & & \\
\hline S95T003284 & \multirow[t]{2}{*}{$114: 3$} & Upper $1 / 2$ & 59.90 & 58.50 & 59.20 & & & \\
\hline S95T003285 & & Lower $1 / 2$ & $<84.5$ & $<96.6$ & $<90.1$ & & & \\
\hline S95T003286 & \multirow[t]{2}{*}{$114: 4$} & Upper $1 / 2$ & 73.00 & 45.60 & 59.30 & & & \\
\hline S95T003287 & & Lower $1 / 2$ & $<17.7$ & $<22.1$ & $<19.9$ & & & \\
\hline S95T003288 & \multirow[t]{2}{*}{$114: 5$} & Upper $1 / 2$ & 21.60 & 21.30 & 21.45 & & & \\
\hline S95T003289 & & Lower $1 / 2$ & 42.50 & 42.70 & 42.60 & & & \\
\hline S95T003290 & \multirow[t]{2}{*}{$114: 6$} & Upper $1 / 2$ & 82.60 & 74.60 & 78.60 & & & \\
\hline S95T003291 & & Lower $1 / 2$ & 43.40 & 42.50 & 42.95 & & & \\
\hline \$95T003292 & \multirow[t]{2}{*}{$114: 7$} & Upper $1 / 2$ & 44.50 & 48.60 & 46.55 & & & \\
\hline S95T003293 & & Lower $1 / 2$ & 28.50 & 26.70 & 27.60 & & & \\
\hline S95T003294 & \multirow[t]{2}{*}{$114: 8$} & Upper $1 / 2$ & 440.0 & 189.0 & 314.5 & & & \\
\hline \$95T003295 & & Lower $1 / 2$ & $<10.4$ & $<10.0$ & $<10.2$ & & & \\
\hline S95T003296 & \multirow[t]{2}{*}{$114: 9$} & Upper $1 / 2$ & $<20.7$ & $<23.2$ & $<22,0$ & & & \\
\hline S95T003297 & & Lower $1 / 2$ & $<18.9$ & $<22.5$ & $<20.7$ & & & \\
\hline
\end{tabular}




\begin{tabular}{|c|c|c|c|c|c|c|c|c|}
\hline \multirow[b]{3}{*}{$\operatorname{IOEO} 0^{\circ} 0>$} & \multirow[b]{3}{*}{ e/u } & \multirow[b]{3}{*}{$\varepsilon S \cdot L>$} & So $0^{\circ}>$ & so.s $>$ & $\mathrm{s}_{0}^{\circ} \mathrm{s}>$ & $\mathrm{Ta}$ & $I: t I I$ & SSZEOOLS6S \\
\hline & & & $0.01>$ & $0.01>$ & $0.01>$ & $\mathrm{Td}$ & $2: 211$ & \$86200LS6S \\
\hline & & & $0.01>$ & $0.01>$ & $0.01>$ & Td & $I: Z I I$ & 9L6200LS6S \\
\hline \multirow[t]{11}{*}{. 1} & 9 & 1urfiry & myrgry & 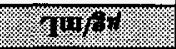 & 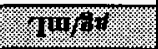 & (1) & \multicolumn{2}{|c|}{ 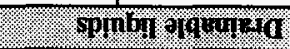 } \\
\hline & & & $202>$ & $I^{\circ} I Z>$ & $\varepsilon^{\circ} 6 \mathrm{I}>$ & $y_{1}$ IOMOT & \multirow[b]{4}{*}{$\varepsilon I: t I I$} & LOEEOOLS6S \\
\hline & & & s.Iz> & $t I z>$ & $9.12>$ & $y / 1$ Iadd $\Lambda$ & & 90EEO0LS6S \\
\hline & & & $2.81>$ & E8I 8 & $0.81>$ & z/ IOMOT & & SOEEOOLS6S \\
\hline & & & $L \cdot I Z>$ & $70 z>$ & $6.22>$ & $3 / 1$ Iadd $n$ & & t0EEOOLS6S \\
\hline & & & $6.51>$ & $5.81>$ & $2 \cdot 11>$ & $2 / 12 \mathrm{MO}$ & \multirow[b]{2}{*}{ ZI:tII } & EOEE00LS6S \\
\hline & & & S.II > & $0.1 \mathrm{II}>$ & $0.21>$ & $y / 1$ Iadd $\Omega$ & & ZOEEOOLS6S \\
\hline & & & $0.2 I>$ & $0 \angle \mathrm{LI}>$ & $0 . L I>$ & $2 / 1$ دәMOT & \multirow[b]{2}{*}{ II } & IOEEOOLS6S \\
\hline & & & $8^{\circ} \varepsilon Z>$ & $I \cdot t z>$ & $S^{*} \varepsilon Z>$ & $y_{1}$ sadd $_{n}$ & & 00EE00LS6S \\
\hline & & & I'8I $>$ & $\varepsilon^{\prime} 6 I>$ & $6.91>$ & $z / 12 M O 7$ & \multirow[b]{2}{*}{$0 I: \nabla I I$} & 66ZE00LS6S \\
\hline & & & $2.8 I>$ & $6.21>$ & S.8I > & $z / 1$ I ddd & & $862 \varepsilon 00 \mathrm{LS} 6 \mathrm{~S}$ \\
\hline$\sqrt{4 \%}$ & 8 & 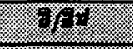 & $8 \%$ & $8.8 \%$ & $97 \%$ & (2) & (2) & $901 \%$ \\
\hline 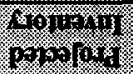 & $\frac{(10 \%}{168 \%}$ & $\begin{array}{l}10901 \% \\
1130010\end{array}$ & 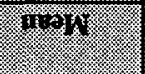 & 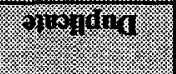 & mist & 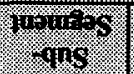 & 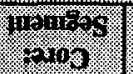 & Timor \\
\hline
\end{tabular}


Table B-5. Tank 241-B-204 Analytical Results: Beryllium. (3 sheets)

\begin{tabular}{|c|c|c|c|c|c|c|c|c|}
\hline 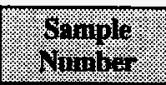 & 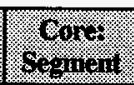 & Sorition & 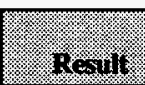 & Biflicis & . Mentis & 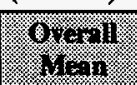 & (ingen: & $\begin{array}{l}\text { Prujeted } \\
\text { Finfentong }\end{array}$ \\
\hline Sollos & 3. & 4 & $4=18 \mathrm{~L}$ & ( 828 & 28 & (2) & , & $18 \mathrm{~g}$ \\
\hline S95T002980 & $112: 2$ & Whole & $<1.38$ & $<1.86$ & $<1.62$ & \multirow[t]{21}{*}{$<2.24$} & \multirow[t]{21}{*}{$n / a$} & \multirow[t]{21}{*}{$<0.493$} \\
\hline S95T003061 & \multirow[t]{2}{*}{$112: 3$} & Upper $1 / 2$ & $<1.20$ & $<0.942$ & $<1.07$ & & & \\
\hline S95T003062 & & Lower $1 / 2$ & $<1.12$ & $<1.06$ & $<1.09$ & & & \\
\hline S95T003063 & \multirow[t]{2}{*}{$112: 4$} & Upper $1 / 2$ & $<0.878$ & $<1.16$ & $<1.02$ & & & \\
\hline S95T003064 & & Lower $1 / 2$ & $<1.21$ & $<1.11$ & $<1.16$ & & & \\
\hline S95T003065 & \multirow[t]{2}{*}{$112: 5$} & Upper $1 / 2$ & $<2.18$ & $<2.08$ & $<2.13$ & & & \\
\hline S95T003066 & & Lower $1 / 2$ & $<1.15$ & $<0.977$ & $<1.06$ & & & \\
\hline S95T003067 & \multirow[t]{2}{*}{$112: 6$} & Upper $1 / 2$ & $<2.33$ & $<1.72$ & $<2.03$ & & & \\
\hline S95T003068 & & Lower $1 / 2$ & $<1.11$ & $<0.969$ & $<1.04$ & & & \\
\hline S95T003069 & \multirow[t]{2}{*}{$112: 7$} & Upper $1 / 2$ & $<1.82$ & $<1.90$ & $<1.86$ & & & \\
\hline S95T003070 & & Lower $1 / 2$ & $<1.76$ & $<1.71$ & $<1.74$ & & & \\
\hline S95T003071 & \multirow[t]{2}{*}{$112: 8$} & Upper $1 / 2$ & $<1.05$ & $<1.15$ & $<1.10$ & & & \\
\hline S95T003072 & & Lower $1 / 2$ & $<1.95$ & $<1.91$ & $<1.93$ & & & \\
\hline S95T003073 & \multirow[t]{2}{*}{$112: 9$} & Upper $1 / 2$ & $<2.07$ & $<1.90$ & $<1.99$ & & & \\
\hline S95T003074 & & Lower $1 / 2$ & $<1.71$ & $<1.91$ & $<1.81$ & & & \\
\hline S95T003075 & \multirow[t]{2}{*}{$112: 10$} & Upper $1 / 2$ & $<1.86$ & $<1.82$ & $<1.84$ & & & \\
\hline S95T003076 & & Lower $1 / 2$ & $<2.28$ & $<2.05$ & $<2.17$ & & & \\
\hline S95T003077 & \multirow[t]{2}{*}{$112: 11$} & Upper $1 / 2$ & $<2.03$ & $<1.74$ & $<1.89$ & & & \\
\hline S95T003078 & & Lower $1 / 2$ & $<4.74$ & $<5.74$ & $<5.24$ & & & \\
\hline S95T003079 & \multirow[t]{2}{*}{$112: 12$} & Upper $1 / 2$ & $<5.35$ & $<4.83$ & $<5.09$ & & & \\
\hline S95T003080 & & Lower $1 / 2$ & $<4.89$ & $<5.04$ & $<4.97$ & & & \\
\hline
\end{tabular}


Table B-5. Tank 241-B-204 Analytical Results: Beryllium. (3 sheets)

\begin{tabular}{|c|c|c|c|c|c|c|c|c|}
\hline 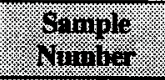 & 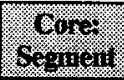 & S. & resuil & 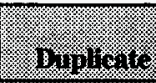 & Hen. & 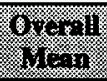 & 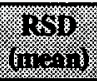 & 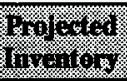 \\
\hline Solvin & & (1) & $8 y$ & (1) & $8 y_{5}$ & 将 & 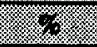 & 28 \\
\hline S95T003081 & \multirow[t]{2}{*}{$112: 13$} & Upper $1 / 2$ & $<4.36$ & $<4.36$ & $<4.36$ & & & \\
\hline S95T003082 & & Lower $1 / 2$ & $<4.86$ & $<4.24$ & $<4.55$ & & & \\
\hline S95T003083 & \multirow[t]{2}{*}{$112: 14$} & Upper $1 / 2$ & $<4.63$ & $<5.29$ & $<4.96$ & & & \\
\hline S95T003084 & & Lower $1 / 2$ & $<9.96$ & $<9.05$ & $<9.51$ & & & \\
\hline S95T003282 & \multirow[t]{2}{*}{$114: 2$} & Upper $1 / 2$ & $<1.05$ & $<1.10$ & $<1.08$ & & & \\
\hline S95T003283 & & Lower $1 / 2$ & $<0.974$ & $<0.910$ & $<0.942$ & & & \\
\hline S95T003284 & \multirow[t]{2}{*}{$114: 3$} & Upper $1 / 2$ & $<0.990$ & $<0.944$ & $<0.967$ & & & \\
\hline S95T003285 & & Lower $1 / 2$ & $<8.45$ & $<9.66$ & $<9.06$ & & & \\
\hline S95T003286 & \multirow[t]{2}{*}{$114: 4$} & Upper $1 / 2$ & $<1.11$ & $<0.984$ & $<1.05$ & & & \\
\hline S95T003287 & & Lower $1 / 2$ & $<1.77$ & $<2.21$ & $<1.99$ & & & \\
\hline S95T003288 & \multirow[t]{2}{*}{$114: 5$} & Upper $1 / 2$ & $<1.11$ & $<0.966$ & $<1.04$ & & & \\
\hline S95T003289 & & Lower $1 / 2$ & $<1.13$ & $<1.17$ & $<1.15$ & & & \\
\hline S95T003290 & \multirow[t]{2}{*}{$114: 6$} & Upper $1 / 2$ & $<1.13$ & $<0.990$ & $<1.06$ & & & \\
\hline S95T003291 & & Lower $1 / 2$ & $<1.72$ & $<2.13$ & $<1.93$ & & & \\
\hline \$95T003292 & \multirow[t]{2}{*}{$114: 7$} & Upper $1 / 2$ & $<1.17$ & $<1.09$ & $<1.13$ & & & \\
\hline S95T003293 & & Lower $1 / 2$ & $<1.08$ & $<0.969$ & $<1.02$ & & & \\
\hline S95T003294 & \multirow[t]{2}{*}{$114: 8$} & Upper $1 / 2$ & $<1.10$ & $<1.79$ & $<1.45$ & & & \\
\hline S95T003295 & & Lower $1 / 2$ & $<1.04$ & $<1.00$ & $<1.02$ & & & \\
\hline S95T003296 & \multirow[t]{2}{*}{$114: 9$} & Upper $1 / 2$ & $<2.07$ & $<2.32$ & $<2.20$ & & & \\
\hline \$95T003297 & & Lower $1 / 2$ & $<1.89$ & $<2.25$ & $<2.07$ & & & \\
\hline
\end{tabular}




\begin{tabular}{|c|c|c|c|c|c|c|c|c|}
\hline \multirow[b]{3}{*}{$\varepsilon 00^{\circ} 0>$} & \multirow[b]{3}{*}{$\mathbf{E} / \mathbf{U}$} & \multirow[b]{3}{*}{$E S L L^{\circ} 0>$} & $0 S^{\circ} 0>$ & $0 S^{\circ} 0>$ & $0 S^{\circ} 0>$ & 7a & I:tII & SSZEOOLS6S \\
\hline & & & $00^{\circ} \mathrm{I}>$ & $00^{\prime} I>$ & $00^{\circ} \mathrm{I}>$ & 70 & Z:ZII & t86200LS6S \\
\hline & & & $00^{\circ} I>$ & $00^{\circ} \mathrm{I}>$ & $00^{\circ} I>$ & Ta & $\mathrm{I}: \mathrm{ZII}$ & $9 \angle 6200 \perp S 6 S$ \\
\hline \multirow[t]{11}{*}{.9} & 9.9: & Tinfl: & (1w & 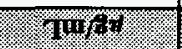 & Ww & 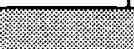 & \multicolumn{2}{|c|}{ spribi arqurier } \\
\hline & & \multirow{10}{*}{. } & $20^{\circ} z>$ & II' $Z>$ & $\mathfrak{E} 6^{\circ} I>$ & $3 / 12 M O T$ & & LOEEOOLS6S \\
\hline & & & sl' $z>$ & $t I^{\circ} \tau>$ & $9 I^{\circ} Z>$ & z/ seddn & $\nabla I: t I I$ & 90EEOOLS6S \\
\hline & & & $28 \cdot 1>$ & E8.I > & $08.1>$ & $2 / 10 M 07$ & \multirow[b]{2}{*}{ EI:†II } & SOEE00LS6S \\
\hline & & & $\angle I Z>$ & $100^{\circ}>$ & $62 \cdot 2>$ & $z_{1}$ lədd $n$ & & TOEEOOLS6S \\
\hline & & & $6 t^{\circ} \mathrm{I}>$ & S8.I $>$ & $2 I^{\circ} I>$ & $2 / 12 \mathrm{MOT}$ & \multirow[b]{2}{*}{$Z I: t I I$} & EOEE00LS6S \\
\hline & & & SI'I > & OI'I > & $0 Z^{\circ} I>$ & $z / 1$ Jodd $n$ & & ZOEE00LS6S \\
\hline & & & $0 L \cdot I>$ & $0 L^{\prime} I>$ & $0 L^{\circ} I>$ & Z/, دOMOT & \multirow[b]{2}{*}{ II:tII } & IOEEOOLS6S \\
\hline & & & $8 \varepsilon^{\prime} Z>$ & It'Z> & $\overline{S E} Z>$ & $z_{1}$ sədd $\Omega$ & & OOEE00LS6S \\
\hline & & & $18.1>$ & $E 6^{\circ} \mathrm{I}>$ & $69^{\circ} I>$ & z/, IOMO7 & \multirow[b]{2}{*}{ OI:DII } & 66ZE00LL6S \\
\hline & & & $28^{\circ} I>$ & $6 L^{\prime} I>$ & $58^{\circ} I>$ & $z / 1$ doddn & & $862 E 00 L S 6 S$ \\
\hline (4y & 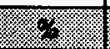 & T/F & STI & 3दि & Why & (3) & & 80108 \\
\hline 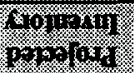 & $\begin{array}{l}\text { proving } \\
\text { asid }\end{array}$ & $\frac{11010}{10+1040}$ & 1360 & 80107 ing & (mosy & 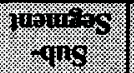 & 10minss: & 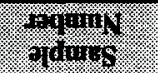 \\
\hline
\end{tabular}


Table B-6. Tank 241-B-204 Analytical Results: Bismuth. (3 sheets)

\begin{tabular}{|c|c|c|c|c|c|c|c|c|}
\hline Hingin & Morer & osing & $\mathrm{h}=\mathrm{s}$ & Oanleme & 38 & 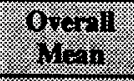 & $\begin{array}{l}x+3 B \\
61+231\end{array}$ & 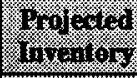 \\
\hline 60110 & 26. & ) & 1968s & 8 & 38 & 30 & . & $x$ \\
\hline S95T002980 & $112: 2$ & Whole & 42,700 & 38,900 & 40,800 & \multirow[t]{19}{*}{48,400} & \multirow[t]{19}{*}{5.8} & \multirow[t]{19}{*}{10,700} \\
\hline S95T003061 & \multirow[t]{2}{*}{$112: 3$} & Upper $1 / 2$ & 48,600 & 50,700 & 49,700 & & & \\
\hline S95T003062 & & Lower $1 / 2$ & 50,000 & 48,800 & 49,400 & & & \\
\hline S95T003063 & \multirow[t]{2}{*}{$112: 4$} & Upper $1 / 2$ & 43,900 & 58,100 & 51,000 & & & \\
\hline S95T003064 & & Lower 1/2 & 61,000 & 61,700 & 61,400 & & & \\
\hline S95T003065 & \multirow[t]{2}{*}{$112: 5$} & Upper $1 / 2$ & 63,100 & 52,200 & 57,600 & & & \\
\hline S95T003066 & & Lower $1 / 2$ & 43,600 & 42,000 & 42,800 & & & \\
\hline S95T003067 & \multirow[t]{2}{*}{$112: 6$} & Upper $1 / 2$ & 47,400 & 49,400 & 48,400 & & & \\
\hline \$95T003068 & & Lower $1 / 2$ & 55,400 & 48,800 & 52,100 & & & \\
\hline S95T003069 & \multirow[t]{2}{*}{$112: 7$} & Upper $1 / 2$ & 50,500 & 56,500 & 53,500 & & & \\
\hline S95T003070 & & Lower $1 / 2$ & 59,500 & 58,900 & 59,200 & & & \\
\hline S95T003071 & \multirow[t]{2}{*}{$112: 8$} & Upper $1 / 2$ & 40,000 & 42,600 & 41,300 & & & \\
\hline S95T003072 & & Lower $1 / 2$ & 49,800 & 48,900 & 49,400 & & & \\
\hline \$95T003073 & \multirow[t]{2}{*}{$112: 9$} & Upper $1 / 2$ & 55,700 & 55,100 & 55,400 & & & \\
\hline S95T003074 & & Lower $1 / 2$ & 47,500 & 49,800 & 48,600 & & & \\
\hline S95T003075 & \multirow[t]{2}{*}{ 112:10 } & Upper $1 / 2$ & 51,200 & 51,200 & 51,200 & & & \\
\hline$\$ 95 T 003076$ & & Lower $1 / 2$ & 48,900 & 50,700 & 49,800 & & & \\
\hline S95T003077 & \multirow[t]{2}{*}{ 112:11 } & Upper $1 / 2$ & 47,500 & 50,000 & 48,800 & & & \\
\hline S95T003078 & & Lower $1 / 2$ & 51,100 & 49,300 & 50,200 & & & \\
\hline
\end{tabular}


Table B-6. Tank 241-B-204 Analytical Results: Bismuth. (3 sheets)

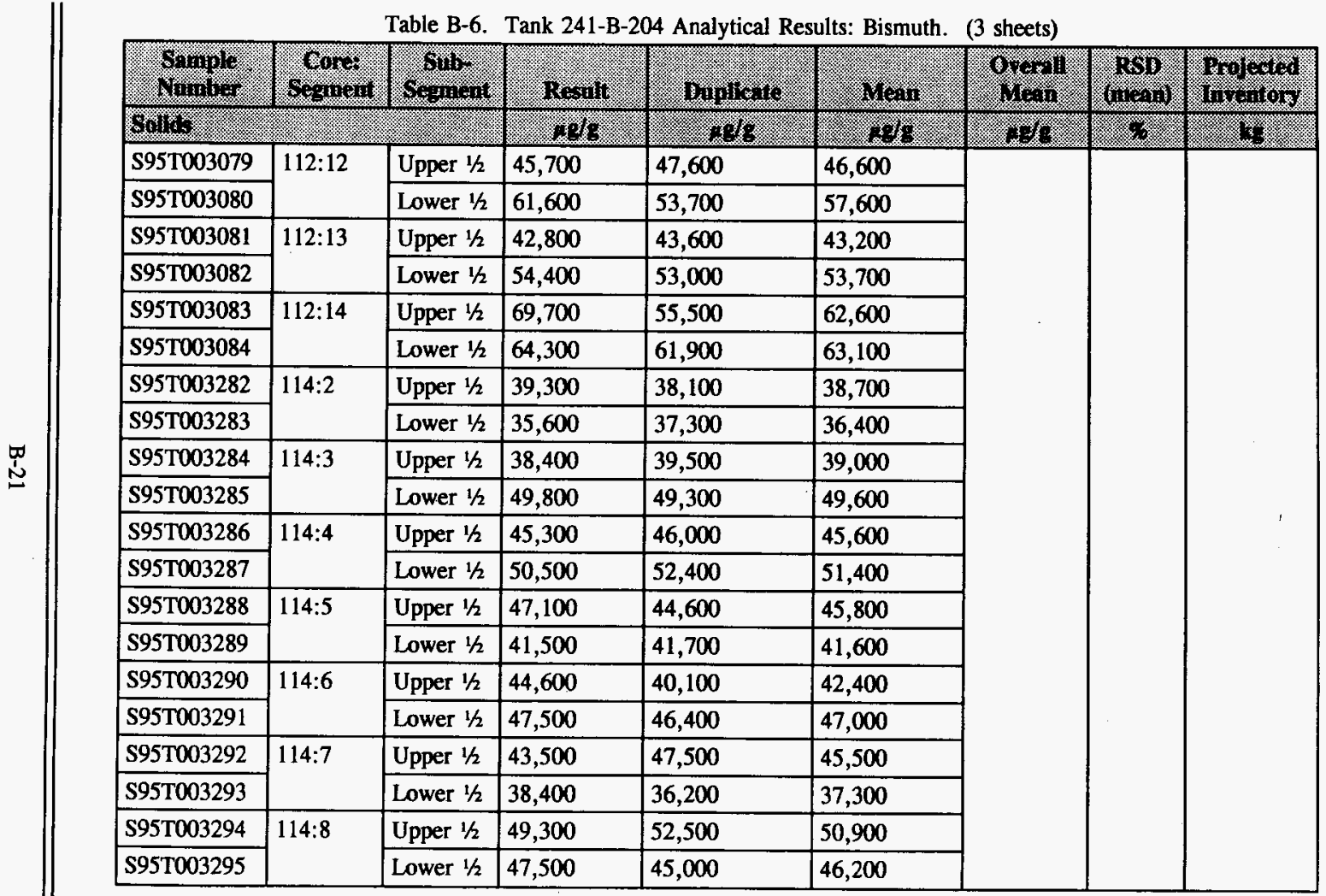


Table B-6. Tank 241-B-204 Analytical Results: Bismuth. (3 sheets)

\begin{tabular}{|c|c|c|c|c|c|c|c|c|}
\hline . & a & 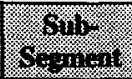 & indil & 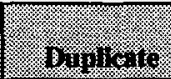 & is & S. & 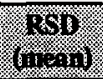 & 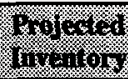 \\
\hline \$95T003296 & \multirow[t]{2}{*}{$114: 9$} & Upper $1 / 2$ & 45,300 & 43,200 & 44,200 & & & \\
\hline \$95T003297 & & Lower $1 / 2$ & 54,900 & 53,300 & 54,100 & & & \\
\hline S95T003300 & \multirow[t]{2}{*}{ 114:11 } & Upper $1 / 2$ & 48,000 & 46,700 & 47,400 & & & \\
\hline$\$ 95 \mathrm{~T} 003301$ & & Lower $1 / 2$ & 48,900 & 47,500 & 48,200 & & & \\
\hline S95T003302 & \multirow[t]{2}{*}{$114: 12$} & Upper $1 / 2$ & 39,100 & 42,500 & 40,800 & & & \\
\hline S95T003303 & & Lower $1 / 2$ & 46,900 & 47,700 & 47,300 & & & \\
\hline S95T003307 & $114: 14$ & Lower $1 / 2$ & 50,300 & 53,300 & 51,800 & & & ' \\
\hline \multicolumn{2}{|c|}{ Draniable logoids? } & 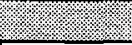 & 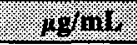 & rog & 1. & $8 \mathrm{~g}$ mI & 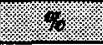 & (2) \\
\hline S95T002976 & 112:1 & DL & $<20.1$ & $<20.1$ & $<20.1$ & \multirow[t]{3}{*}{$<15.1$} & \multirow[t]{3}{*}{$\mathrm{n} / \mathrm{a}$} & \multirow[t]{3}{*}{$<0.0604$} \\
\hline S95T002984 & $112: 2$ & $\mathrm{DL}$ & $<20.1$ & $<20.1$ & $<20.1$ & & & \\
\hline S95T003255 & $114: 1$ & $\mathrm{DL}$ & $<10.1$ & $<10.1$ & $<10.1$ & & & \\
\hline
\end{tabular}




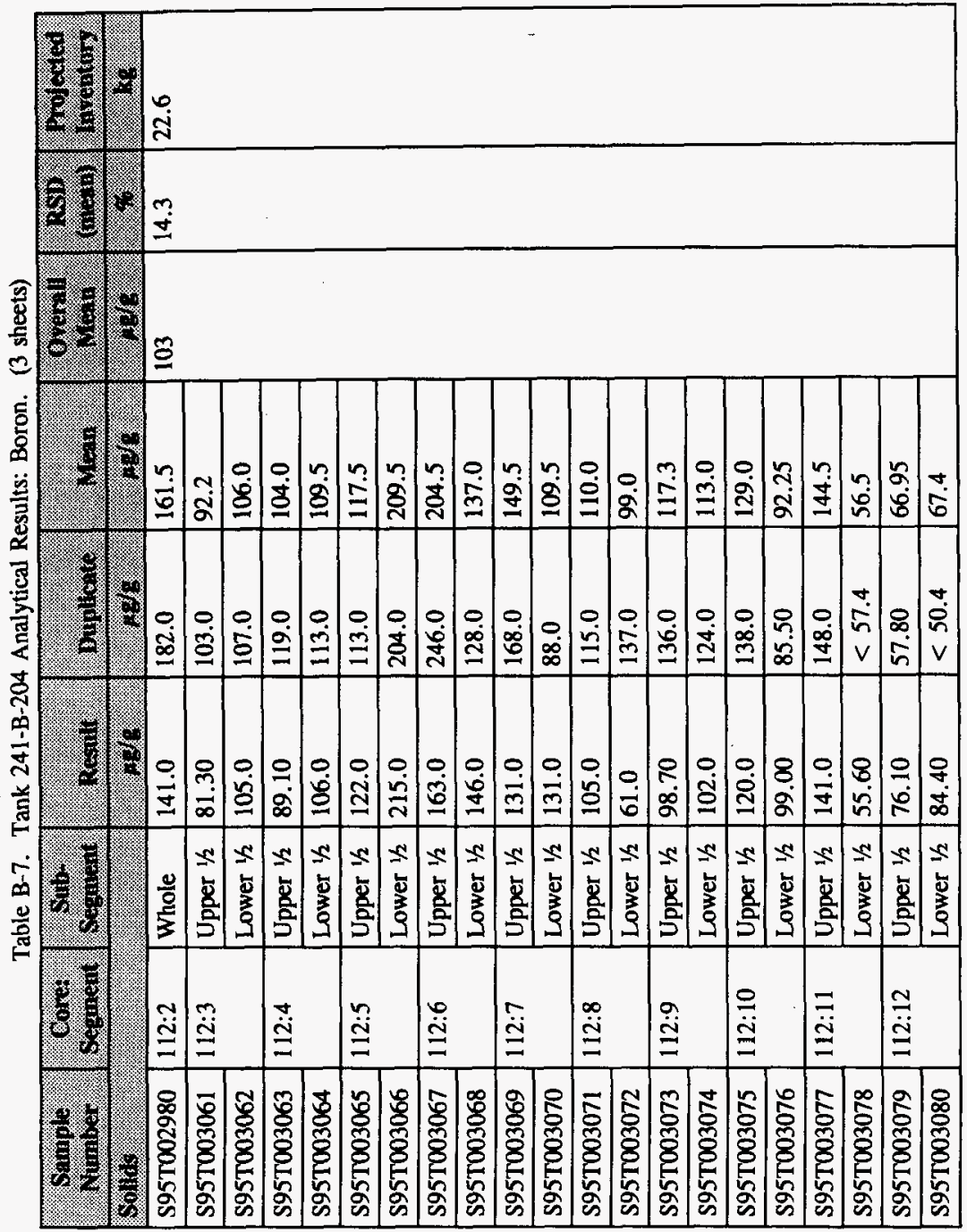




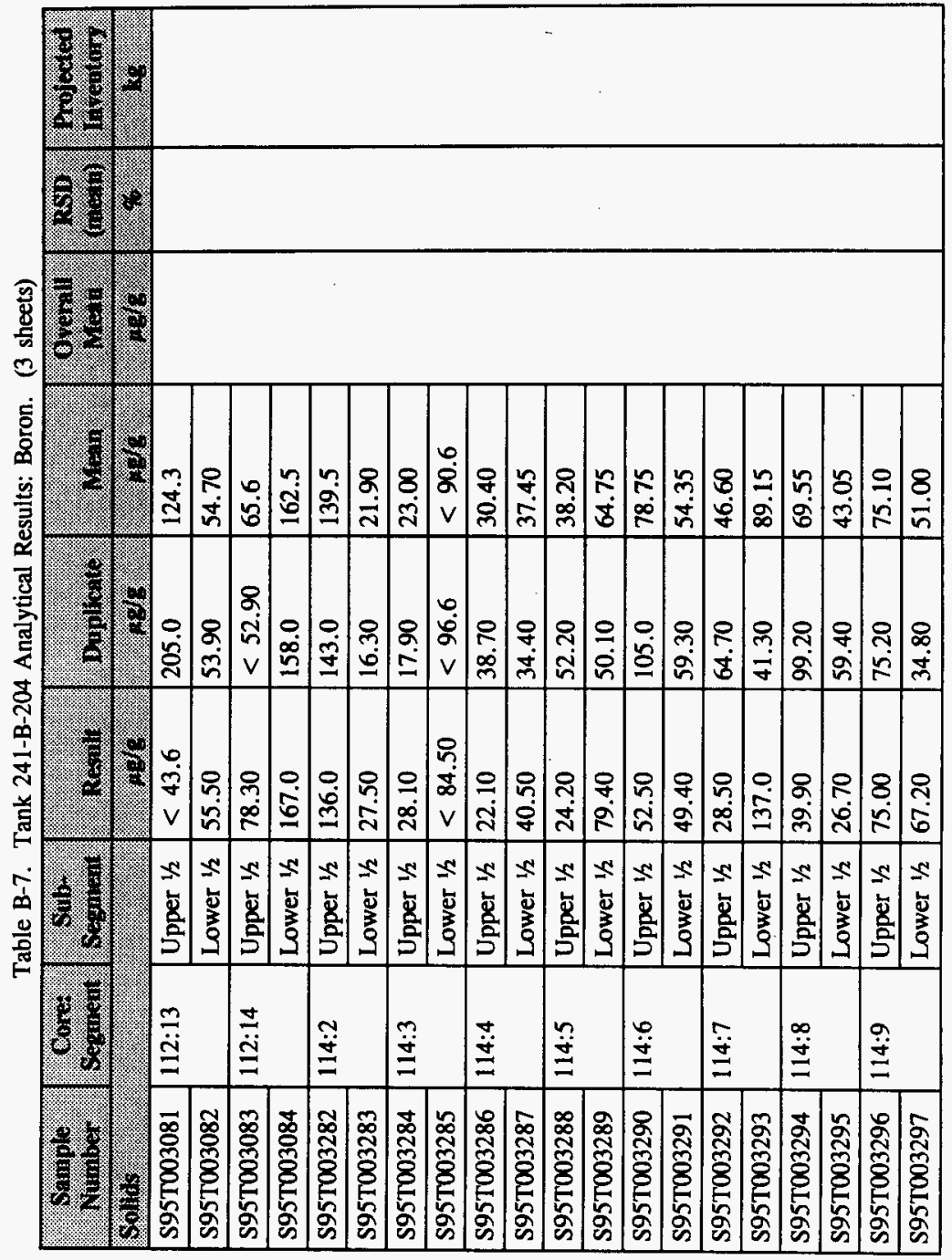


Table B-7. Tank 241-B-204 Analytical Results: Boron. (3 sheets)

\begin{tabular}{|c|c|c|c|c|c|c|c|c|}
\hline Wing & Worther & 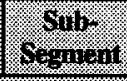 & Menull: & B.pilone & 1) & 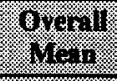 & 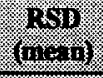 & 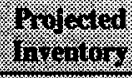 \\
\hline \multicolumn{3}{|l|}{ Solis } & 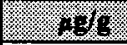 & (1) & $.0 \%$ & 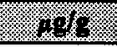 & 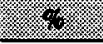 & 8 \\
\hline S95T003298 & \multirow[t]{2}{*}{$114: 10$} & Upper $1 / 2$ & 54.10 & 37.60 & 45.85 & & & \\
\hline \$95T003299 & & Lower $1 / 2$ & 123.0 & 129.0 & 126.0 & & & \\
\hline S95T003300 & \multirow[t]{2}{*}{ 114:11 } & Upper $1 / 2$ & 150.0 & 150.0 & 150.0 & & & \\
\hline S95T003301 & & Lower $1 / 2$ & 131.0 & 119.0 & 125.0 & & & \\
\hline S95T003302 & \multirow[t]{2}{*}{$114: 12$} & Upper $1 / 2$ & 135.0 & 142.0 & 138.5 & & & \\
\hline S95T003303 & & Lower $1 / 2$ & 132.0 & 138.0 & 135.0 & & & \\
\hline S95T003304 & \multirow[t]{2}{*}{$114: 13$} & Upper $1 / 2$ & 162.0 & 139.0 & 150.5 & & & \\
\hline S95T003305 & & Lower $1 / 2$ & 165.0 & 137.0 & 151.0 & & & \\
\hline S95T003306 & \multirow[t]{2}{*}{$114: 14$} & Upper $1 / 2$ & 143.0 & 163.0 & 153.0 & & & \\
\hline \$95T003307 & & Lower $1 / 2$ & 125.0 & 170.0 & 147.5 & & & \\
\hline \multicolumn{3}{|c|}{ 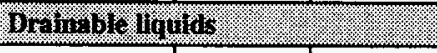 } & $4 \mathrm{~g} / \mathrm{ni}$ & a $\sin$ & (c) & 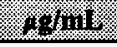 & 9. & \% \\
\hline S95T002976 & 112:1 & DL & $<10.0$ & $<10.0$ & $<10.0$ & \multirow[t]{3}{*}{$<7.53$} & \multirow[t]{3}{*}{$n / a$} & \multirow[t]{3}{*}{$<0.0301$} \\
\hline S95T002984 & $112: 2$ & $\mathrm{DL}$ & $<10.0$ & $<10.0$ & $<10.0$ & & & \\
\hline S95T003255 & 114:1 & DL & $<5.05$ & $<5.05$ & $<5.05$ & & & \\
\hline
\end{tabular}




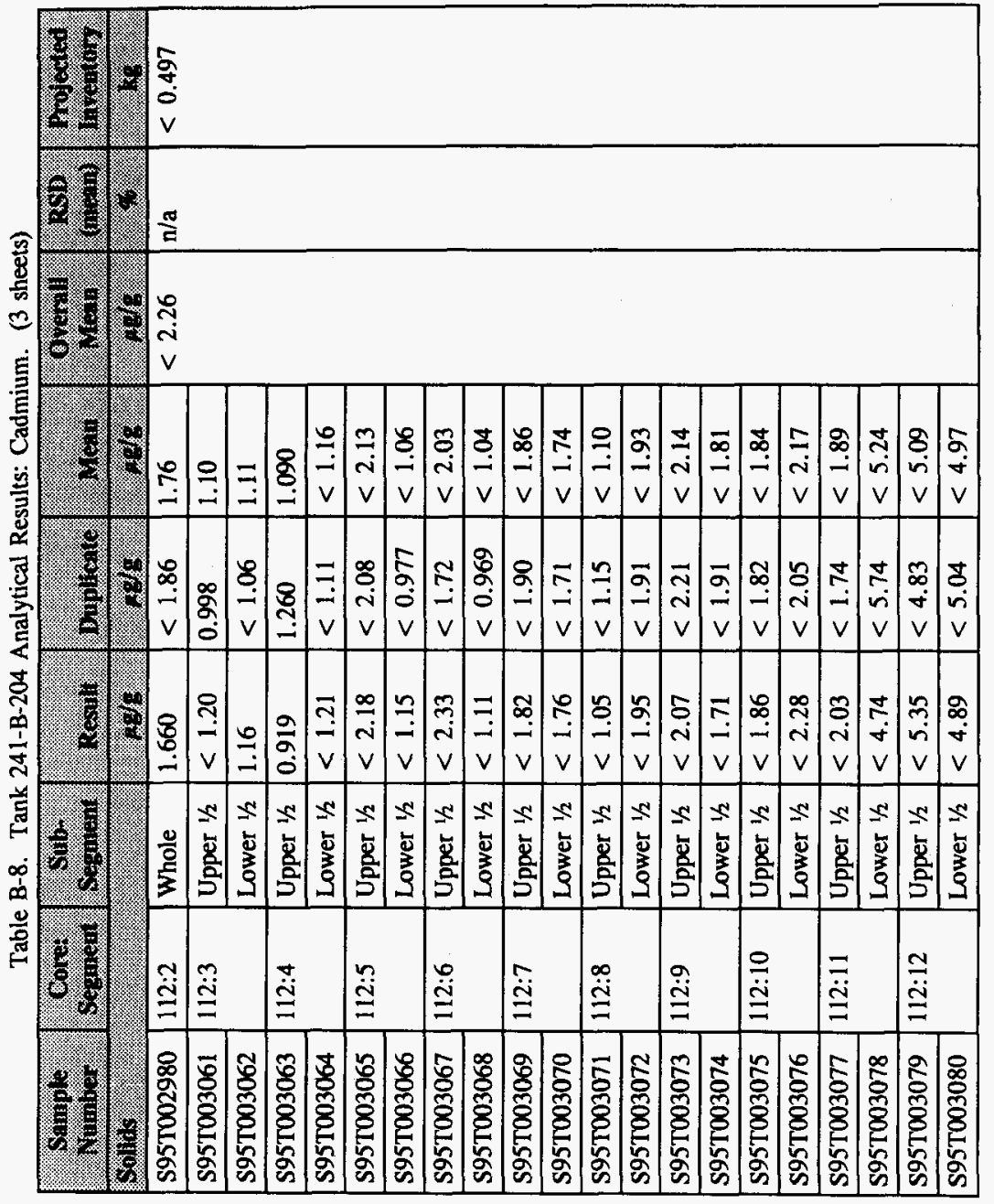


Table B-8. Tank 241-B-204 Analytical Results: Cadmium. (3 sheets)

\begin{tabular}{|c|c|c|c|c|c|c|c|c|}
\hline $\begin{array}{l}\text { Sample } \\
\text { rumber }\end{array}$ & 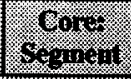 & sub & Rosing & $(34 y)$ sing & 3. & 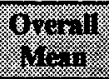 & 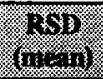 & 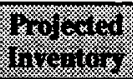 \\
\hline Solous & (2) & (5) & 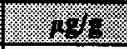 & 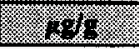 & 7.48 & 1.006 & 4. & 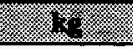 \\
\hline S95T003081 & \multirow[t]{2}{*}{$112: 13$} & Upper $1 / 2$ & $<4.36$ & $<4.36$ & $<4.36$ & & & \\
\hline S95T003082 & & Lower $1 / 2$ & $<4.86$ & $<4.24$ & $<4.55$ & & & \\
\hline S95T003083 & \multirow[t]{2}{*}{$112: 14$} & Upper $1 / 2$ & $<4.63$ & $<5.29$ & $<4.96$ & & & \\
\hline S95T003084 & & Lower $1 / 2$ & $<9.96$ & $<9.05$ & $<9.51$ & & & \\
\hline S95T003282 & \multirow[t]{2}{*}{$114: 2$} & Upper $1 / 2$ & $<1.05$ & $<1.10$ & $<1.08$ & & & \\
\hline S95T003283 & & Lower $1 / 2$ & $<0.974$ & $<0.910$ & $<0.942$ & & & \\
\hline S95T003284 & \multirow[t]{2}{*}{$114: 3$} & Upper $1 / 2$ & $<0.990$ & $<0.944$ & $<0.967$ & & & \\
\hline S95T003285 & & Lower $1 / 2$ & $<8.45$ & $<9.66$ & $<9.06$ & & & \\
\hline S95T003286 & \multirow[t]{2}{*}{$114: 4$} & Upper $1 / 2$ & $<1.11$ & $<0.984$ & $<1.05$ & & & \\
\hline S95T003287 & & Lower $1 / 2$ & $<1.77$ & $<2.21$ & $<1.99$ & & & \\
\hline S95T003288 & \multirow[t]{2}{*}{$114: 5$} & Upper $1 / 2$ & $<1.11$ & $<0.966$ & $<1.04$ & & & \\
\hline S95T003289 & & Lower $1 / 2$ & $<1.13$ & $<1.17$ & $<1.15$ & & & \\
\hline S95T003290 & \multirow[t]{2}{*}{$114: 6$} & \begin{tabular}{|l|} 
Upper $1 / 2$ \\
\end{tabular} & 1.280 & 1.390 & 1.335 & & & \\
\hline S95T003291 & & Lower $1 / 2$ & $<1.72$ & $<2.13$ & $<1.93$ & & & \\
\hline S95T003292 & \multirow[t]{2}{*}{$114: 7$} & Upper $1 / 2$ & $<1.17$ & $<1.09$ & $<1.13$ & & & \\
\hline S95T003293 & & Lower $1 / 2$ & $<1.08$ & $<0.969$ & $<1.02$ & & & \\
\hline S95T003294 & \multirow[t]{2}{*}{$114: 8$} & Upper $1 / 2$ & $<1.10$ & $<1.79$ & $<1.45$ & & & \\
\hline S95T003295 & & Lower $1 / 2$ & 1.180 & $<1.00$ & $<1.09$ & & & \\
\hline S95T003296 & \multirow[t]{2}{*}{$114: 9$} & Upper $1 / 2$ & $<2.07$ & $<2.32$ & $<2.20$ & & & \\
\hline S95T003297 & & Lower $1 / 2$ & 2.000 & $<2.25$ & $<2.13$ & & & \\
\hline
\end{tabular}


Table B-8. Tank 241-B-204 Analytical Results: Cadmium. (3 sheets)

\begin{tabular}{|c|c|c|c|c|c|c|c|c|}
\hline oinol & 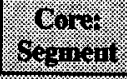 & (t) & ravil: & $g_{4}(3)$ & 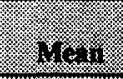 & (1) & 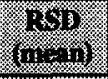 & 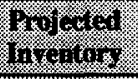 \\
\hline Solis:s. & 4. & (2) & 1945 & .915 & 4. & 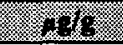 & 2. & IIs \\
\hline S95T003298 & \multirow[t]{2}{*}{$114: 10$} & Upper $1 / 2$ & $<1.85$ & $<1.79$ & $<1.82$ & & & \\
\hline \$95T003299 & & Lower $1 / 2$ & $<1.69$ & $<1.93$ & $<1.81$ & & & \\
\hline S95T003300 & \multirow[t]{2}{*}{$114: 11$} & Upper $1 / 2$ & $<2.35$ & $<2.41$ & $<2.38$ & & & \\
\hline S95T003301 & & Lower $1 / 2$ & $<1.70$ & $<1.70$ & $<1.70$ & & & \\
\hline S95T003302 & \multirow[t]{2}{*}{$114: 12$} & Upper $1 / 2$ & $<1.20$ & $<1.10$ & $<1.15$ & & & \\
\hline s95T003303 & & Lower $1 / 2$ & $<1.12$ & $<1.85$ & $<1.49$ & & & \\
\hline S95T003304 & \multirow[t]{2}{*}{$114: 13$} & Upper $1 / 2$ & $<2.29$ & $<2.04$ & $<2.17$ & & & \\
\hline \$95T003305 & & Lower $1 / 2$ & $<1.80$ & $<1.83$ & $<1.82$ & & & \\
\hline S95T003306 & \multirow[t]{2}{*}{$114: 14$} & Upper $1 / 2$ & $<2.16$ & $<2.14$ & $<2.15$ & & & \\
\hline S95T003307 & & Lower $1 / 2$ & $<1.93$ & $<2.11$ & $<2.02$ & & & \\
\hline \multicolumn{3}{|c|}{ Oraming ingus } & $49 \mathrm{mi}$ & $y_{11 i}$ & \%il & $=23 m_{11}$ & (x) & 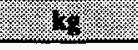 \\
\hline S95T002976 & $112: 1$ & $\mathrm{DL}$ & $<1.00$ & $<1.00$ & $<1.00$ & \multirow[t]{3}{*}{$<0.753$} & \multirow[t]{3}{*}{$n / a$} & \multirow[t]{3}{*}{$<0.00301$} \\
\hline S95T002984 & $112: 2$ & $\mathrm{DL}$ & $<1.00$ & $<1.00$ & $<1.00$ & & & \\
\hline S95T003255 & $114: 1$ & $\mathrm{DL}$ & $<0.505$ & $<0.505$ & $<0.505$ & & & \\
\hline
\end{tabular}




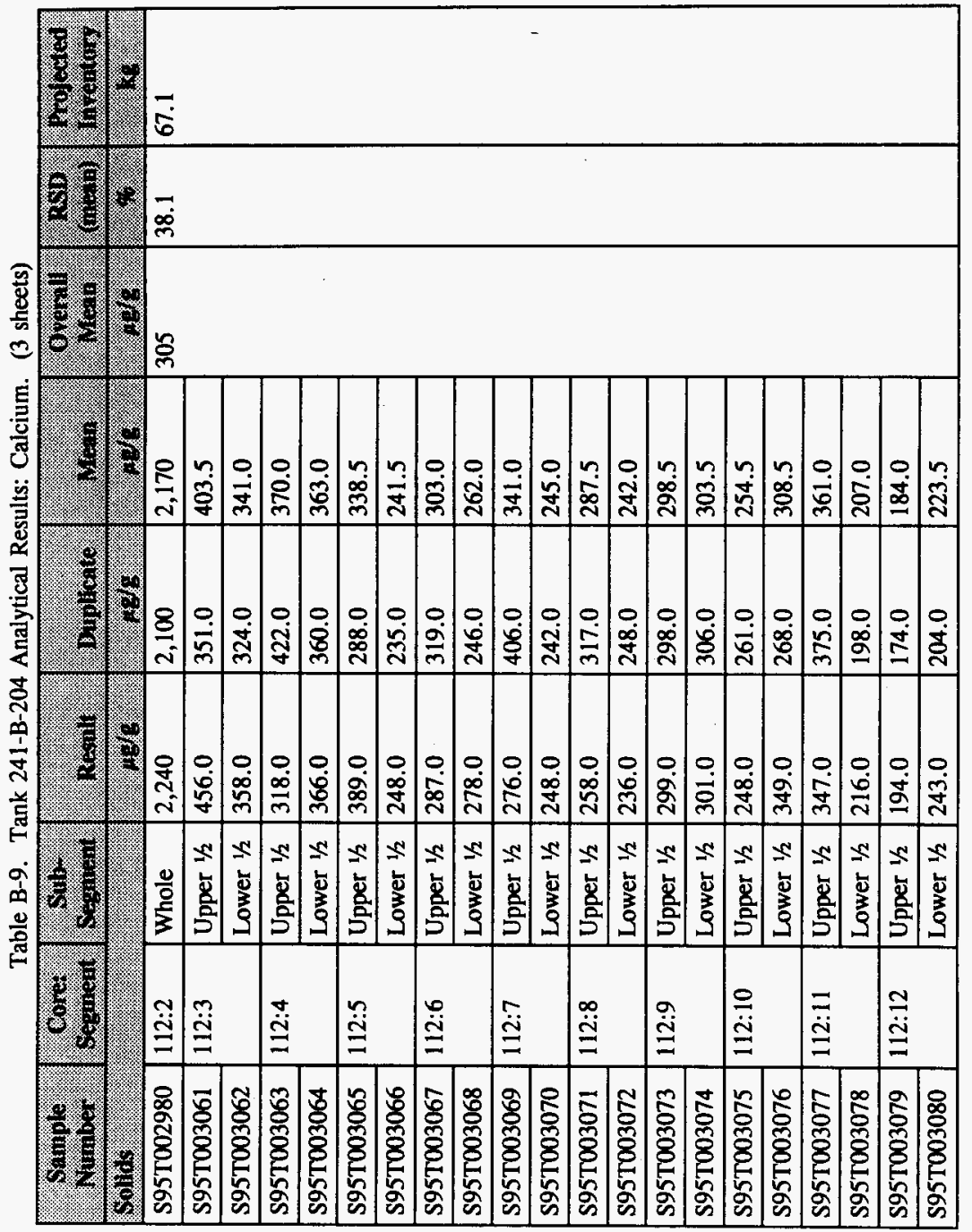




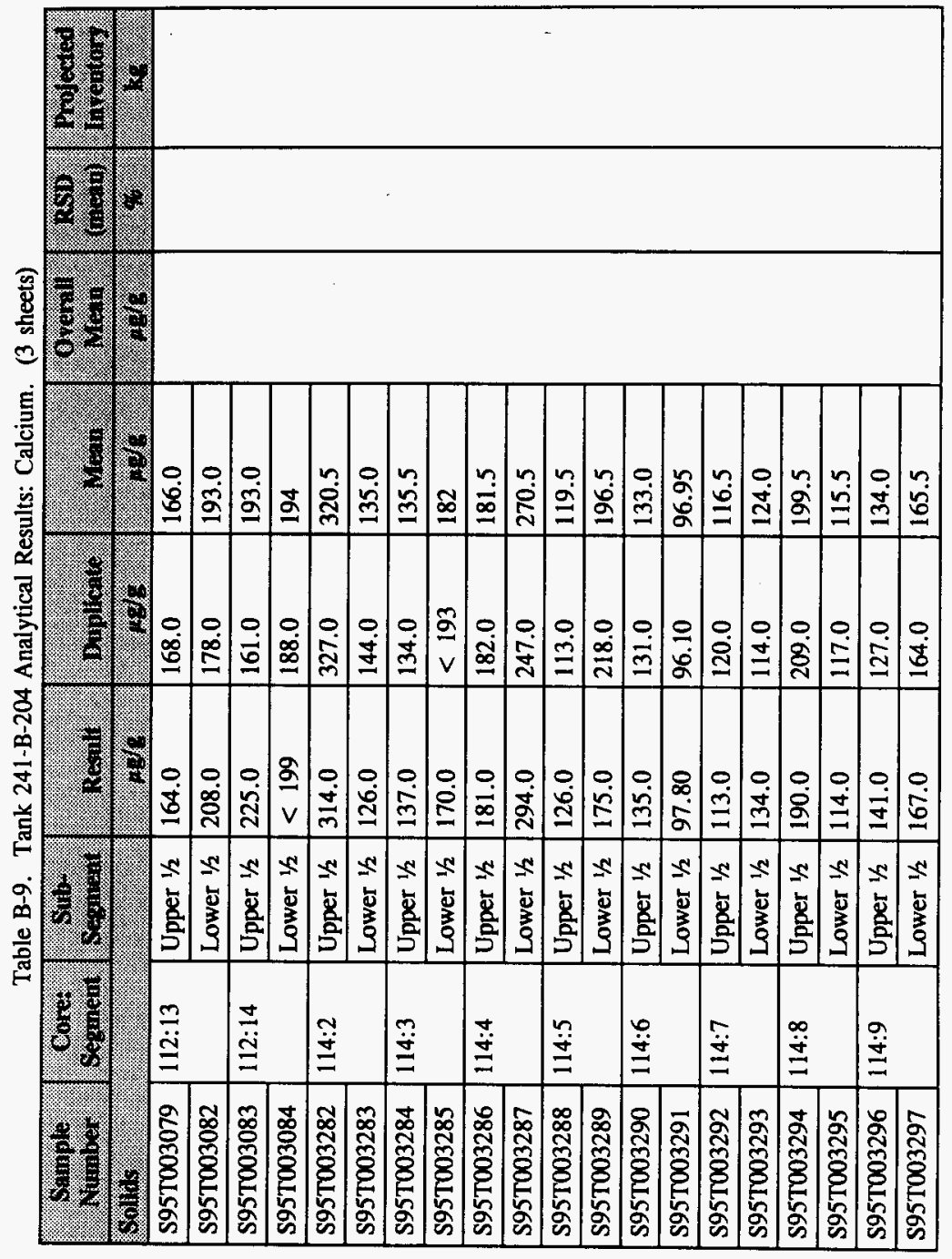


Table B-9. Tank 241-B-204 Analytical Results: Calcium. (3 sheets)

\begin{tabular}{|c|c|c|c|c|c|c|c|c|}
\hline Mong & hespon & 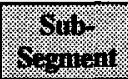 & alcinil & 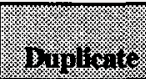 & 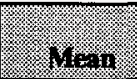 & (6) & 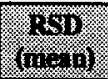 & 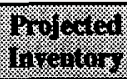 \\
\hline $6016 \%$ & & 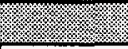 & 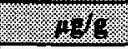 & 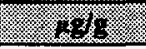 & m. & $y_{3}$ & 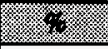 & (1) \\
\hline S95T003298 & \multirow[t]{2}{*}{$114: 10$} & Upper $1 / 2$ & 150.0 & 148.0 & 149.0 & & & \\
\hline S95T003299 & & Lower $1 / 2$ & 212.0 & 175.0 & 193.5 & & & \\
\hline S95T003300 & \multirow[t]{2}{*}{$114: 11$} & Upper $1 / 2$ & 170.0 & 169.0 & 169.5 & & & \\
\hline S95T003301 & & Lower $1 / 2$ & 174.0 & 221.0 & 197.5 & & & \\
\hline S95T003302 & \multirow[t]{2}{*}{$114: 12$} & Upper $1 / 2$ & 170.0 & 145.0 & 157.5 & & & \\
\hline S95T003303 & & Lower $1 / 2$ & 155.0 & 151.0 & 153.0 & & & \\
\hline S95T003304 & \multirow[t]{2}{*}{$114: 13$} & Upper $1 / 2$ & 225.0 & 237.0 & 231.0 & & & \\
\hline S95T003305 & & Lower $1 / 2$ & 280.0 & 228.0 & 254.0 & & & \\
\hline S95T003306 & \multirow[t]{2}{*}{$114: 14$} & Upper $1 / 2$ & 298.0 & 369.0 & 333.5 & & & \\
\hline S95T003307 & & Lower $1 / 2$ & 521.0 & 347.0 & 434.0 & & & \\
\hline \multicolumn{3}{|c|}{ Braingle Gruds } & 1.1/111 & 40 & aldint & (1) & 样 & 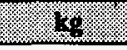 \\
\hline S95T002976 & $112: 1$ & DL & $<20.1$ & $<20.1$ & $<20.1$ & \multirow[t]{3}{*}{$<15.1$} & \multirow[t]{3}{*}{$n / a$} & \multirow[t]{3}{*}{$<0.0604$} \\
\hline S95T002984 & $112: 2$ & DL & $<20.1$ & $<20.1$ & $<20.1$ & & & \\
\hline S95T003255 & $114: 1$ & DL & $<10.1$ & $<10.1$ & $<10.1$ & & & \\
\hline
\end{tabular}


Table B-10. Tank 241-B-204 Analytical Results: Cerium. (3 sheets)

\begin{tabular}{|c|c|c|c|c|c|c|c|c|}
\hline Womp & 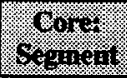 & 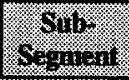 & Rowill & Hiplizar & (y) & 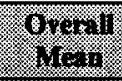 & 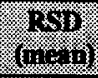 & 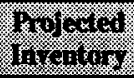 \\
\hline Comos. & (4: & ; & 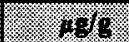 & $(27)$ & 380 & 1.96 & s. & 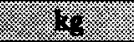 \\
\hline S95T002980 & $112: 2$ & Whole & $<27.6$ & $<37.1$ & $<32.4$ & \multirow[t]{21}{*}{55.4} & \multirow[t]{21}{*}{13.3} & \multirow[t]{21}{*}{12.2} \\
\hline S95T003061 & \multirow[t]{2}{*}{$112: 3$} & Upper $1 / 2$ & 44.50 & 47.60 & 46.05 & & & \\
\hline \$95T003062 & & Lower $1 / 2$ & 59.20 & 57.10 & 58.15 & & & \\
\hline S95T003063 & \multirow[t]{2}{*}{$112: 4$} & Upper $1 / 2$ & 47.70 & 62.30 & 55.00 & & & \\
\hline \$95T003064 & & Lower $1 / 2$ & 64.20 & 68.80 & 66.50 & & & \\
\hline S95T003065 & \multirow[t]{2}{*}{$112: 5$} & Upper $1 / 2$ & 62.20 & 48.50 & 55.35 & & & \\
\hline S95T003066 & & Lower $1 / 2$ & 47.50 & 43.00 & 45.25 & & & \\
\hline S95T003067 & \multirow[t]{2}{*}{$112: 6$} & Upper $1 / 2$ & 49.50 & 52.40 & 50.95 & & & \\
\hline S95T003068 & & Lower $1 / 2$ & 45.30 & 39.60 & 42.45 & & & \\
\hline \$95T003069 & \multirow[t]{2}{*}{$112: 7$} & Upper $1 / 2$ & 47.90 & 52.50 & 50.20 & & & \\
\hline S95T003070 & & Lower $1 / 2$ & 52.90 & 50.10 & 51.50 & & & \\
\hline S95T003071 & \multirow[t]{2}{*}{$112: 8$} & Upper $1 / 2$ & 25.90 & 30.30 & 28.10 & & & \\
\hline S95T003072 & & Lower $1 / 2$ & $<39.1$ & $<38.1$ & $<38.6$ & & & \\
\hline S95T003073 & \multirow[t]{2}{*}{$112: 9$} & Upper $1 / 2$ & 42.20 & 41.20 & 41.70 & & & \\
\hline \$95T003074 & & Lower $1 / 2$ & $<34.3$ & $<38.1$ & $<36.2$ & & & \\
\hline S95T003075 & \multirow[t]{2}{*}{$112: 10$} & Upper $1 / 2$ & $<37.2$ & $<36.4$ & $<36.8$ & & & \\
\hline S95T003076 & & Lower $1 / 2$ & $<45.6$ & $<41.1$ & $<43.4$ & & & \\
\hline S95T003077 & \multirow[t]{2}{*}{$112: 11$} & Upper $1 / 2$ & $<40.7$ & $<34.8$ & $<37.8$ & & & \\
\hline S95T003078 & & Lower $1 / 2$ & $<94.9$ & $<115$ & $<105$ & & & \\
\hline \$95T003079 & \multirow[t]{2}{*}{$112: 12$} & Upper $1 / 2$ & $<107$ & $<96.6$ & $<102$ & & & \\
\hline S95T003080 & & Lower $1 / 2$ & $<97.8$ & $<101$ & $<99.4$ & & & \\
\hline
\end{tabular}


Table B-10. Tank 241-B-204 Analytical Results: Cerium. (3 sheets)

\begin{tabular}{|c|c|c|c|c|c|c|c|c|}
\hline 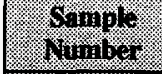 & orotion & $\log 10$ & Q a & 1) & $314=$ & $8 \%$ & $\frac{(2983}{(19-13)}$ & 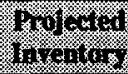 \\
\hline Sollos & & (2.8. & 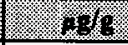 & 28 & bese & 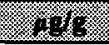 & 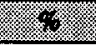 & 86 \\
\hline S95T003081 & \multirow[t]{2}{*}{$112: 13$} & Upper $1 / 2$ & $<87.2$ & $<87.3$ & $<87.3$ & & & \\
\hline S95T003082 & & Lower $1 / 2$ & $<97.2$ & $<84.7$ & $<91.0$ & & & \\
\hline S95T003083 & \multirow[t]{2}{*}{ 112:14 } & Upper $1 / 2$ & $<92.6$ & $<106$ & $<99.3$ & & & \\
\hline S95T003084 & & Lower $1 / 2$ & $<199$ & $<181$ & $<190$ & & & \\
\hline S95T003282 & \multirow[t]{2}{*}{$114: 2$} & Upper $1 / 2$ & 48.30 & 46.40 & 47.35 & & & \\
\hline S95T003283 & & Lower $1 / 2$ & 45.30 & 48.50 & 46.90 & & & \\
\hline S95T003284 & \multirow[t]{2}{*}{$114: 3$} & Upper $1 / 2$ & 43.6 & 42.0 & 42.80 & & & \\
\hline S95T003285 & & Lower $1 / 2$ & $<169$ & $<193$ & $<181$ & & & \\
\hline S95T003286 & \multirow[t]{2}{*}{$114: 4$} & Upper $1 / 2$ & 49.30 & 48.70 & 49.00 & & & \\
\hline S95T003287 & & Lower $1 / 2$ & 47.10 & 44.30 & 45.70 & & & \\
\hline S95T003288 & \multirow[t]{2}{*}{$114: 5$} & Upper $1 / 2$ & 31.00 & 28.20 & 29.60 & & & \\
\hline S95T003289 & & Lower $1 / 2$ & 43.00 & 41.50 & 42.25 & & & \\
\hline S95T003290 & \multirow[t]{2}{*}{$114: 6$} & Upper $1 / 2$ & 42.90 & 39.50 & 41.20 & & & \\
\hline S95T003291 & & Lower $1 / 2$ & $<34.4$ & $<42.5$ & 38.5 & & & \\
\hline S95T003292 & \multirow[t]{2}{*}{$114: 7$} & Upper $1 / 2$ & 46.3 & 49.20 & 47.75 & & & \\
\hline S95T003293 & & Lower $1 / 2$ & 44.10 & 36.80 & 40.45 & & & \\
\hline S95T003294 & \multirow[t]{2}{*}{$114: 8$} & Upper $1 / 2$ & 32.00 & $<35.8$ & 33.9 & & & \\
\hline S95T003295 & & Lower $1 / 2$ & 32.50 & 34.50 & 33.50 & & & \\
\hline S95T003296 & \multirow[t]{2}{*}{$114: 9$} & Upper $1 / 2$ & 49.70 & $<46.4$ & 48.1 & & & \\
\hline S95T003297 & & Lower $1 / 2$ & 44.20 & $<45.0$ & 44.6 & & & \\
\hline
\end{tabular}


Table B-10. Tank 241-B-204 Analytical Results: Cerium. (3 sheets)

\begin{tabular}{|c|c|c|c|c|c|c|c|c|}
\hline Simorer & $6 \log ^{2}$ & (4) & monill & 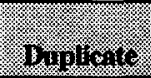 & $y=18$ & 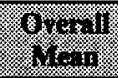 & 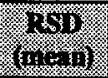 & How ching \\
\hline $601 y_{5}$ & & \% & 1190 & 4.96 & $8 \mathrm{~g}$ & $8 \%$ & (4) & 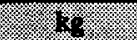 \\
\hline S95T003298 & \multirow[t]{2}{*}{$114: 10$} & Upper $1 / 2$ & $<37.0$ & $<35.9$ & $<36.5$ & & & \\
\hline S95T003299 & & Lower $1 / 2$ & $<33.9$ & $<38.5$ & $<36.2$ & & & \\
\hline S95T003300 & \multirow[t]{2}{*}{$114: 11$} & Upper $1 / 2$ & $<47.0$ & $<48.3$ & $<47.7$ & & & \\
\hline S95T003301 & & Lower $1 / 2$ & $<34.0$ & $<34.0$ & $<34.0$ & & & \\
\hline S95T003302 & \multirow[t]{2}{*}{$114: 12$} & Upper $1 / 2$ & 30.20 & 31.00 & 30.60 & & & \\
\hline \$95T003303 & & Lower $1 / 2$ & 37.30 & 37.30 & 37.30 & & & \\
\hline S95T003304 & \multirow[t]{2}{*}{$114: 13$} & Upper $1 / 2$ & 56.20 & 62.60 & 59.40 & & & \\
\hline \$95T003305 & & Lower $1 / 2$ & 50.30 & 50.30 & 50.30 & & & \\
\hline S95T003306 & \multirow[t]{2}{*}{$114: 14$} & Upper $1 / 2$ & 49.00 & 50.10 & 49.55 & & & \\
\hline S95T003307 & & Lower $1 / 2$ & 61.40 & 65.30 & 63.35 & & & \\
\hline \multicolumn{2}{|c|}{ Brativol hinuls } & (3. & 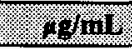 & (2) & $(2,2111$ & (1) \%int & s) & 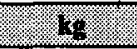 \\
\hline S95T002976 & 112:1 & $\mathrm{DL}$ & $<20.1$ & $<20.1$ & $<20.1$ & \multirow[t]{3}{*}{$<15.1$} & \multirow[t]{3}{*}{$n / a$} & \multirow[t]{3}{*}{$<0.0604$} \\
\hline S95T002984 & $112: 2$ & DL & $<20.1$ & $<20.1$ & $<20.1$ & & & \\
\hline \$95T003255 & $114: 1$ & DL & $<10.1$ & $<10.1$ & $<10.1$ & & & \\
\hline
\end{tabular}


Table B-11. Tank 241-B-204 Analytical Results: Chromium. (3 sheets)

\begin{tabular}{|c|c|c|c|c|c|c|c|c|}
\hline \%olinges & oror & 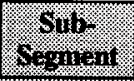 & Imanin & 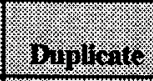 & 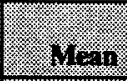 & (6) & 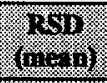 & matengo \\
\hline oolos & & & 40 & 1048 & 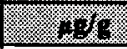 & 13: & . & lo \\
\hline S95T002980 & $112: 2$ & Whole & 1,190 & 1,110 & 1,150 & \multirow[t]{21}{*}{3,240} & \multirow[t]{21}{*}{5.7} & \multirow[t]{21}{*}{714} \\
\hline S95T003061 & \multirow[t]{2}{*}{$112: 3$} & Upper $1 / 2$ & 1,790 & 1,880 & 1,840 & & & \\
\hline S95T003062 & & Lower $1 / 2$ & 2,280 & 2,220 & 2,250 & & & \\
\hline S95T003063 & \multirow[t]{2}{*}{$112: 4$} & Upper $1 / 2$ & 1,990 & 2,630 & 2,310 & & & \\
\hline S95T003064 & & Lower $1 / 2$ & 2,360 & 2,390 & 2,380 & & & \\
\hline S95T003065 & \multirow[t]{2}{*}{$112: 5$} & Upper $1 / 2$ & 2,950 & 2,550 & 2,750 & & & \\
\hline S95T003066 & & Lower $1 / 2$ & 2,580 & 2,530 & 2,560 & & & \\
\hline S95T003067 & \multirow[t]{2}{*}{$112: 6$} & Upper $1 / 2$ & 3,040 & 3,150 & 3,100 & & & \\
\hline S95T003068 & & Lower $1 / 2$ & 4,100 & 3,630 & 3,860 & & & \\
\hline S95T003069 & \multirow[t]{2}{*}{$112: 7$} & Upper $1 / 2$ & 3,970 & 4,260 & 4,120 & & & \\
\hline S95T003070 & & Lower $1 / 2$ & 4,010 & 3,930 & 3,970 & & & \\
\hline S95T003071 & \multirow[t]{2}{*}{$112: 8$} & Upper $1 / 2$ & 3,360 & 3,440 & 3,400 & & & \\
\hline S95T003072 & & Lower $1 / 2$ & 4,420 & 4,490 & 4,460 & & & \\
\hline S95T003073 & \multirow[t]{2}{*}{$112: 9$} & Upper $1 / 2$ & 4,700 & 4,650 & 4,680 & & & \\
\hline S95T003074 & & Lower $1 / 2$ & 3,530 & 3,690 & 3,610 & & & \\
\hline S95T003075 & \multirow[t]{2}{*}{$112: 10$} & Upper $1 / 2$ & 3,680 & 3,710 & 3,700 & & & \\
\hline S95T003076 & & Lower $1 / 2$ & 4,170 & 4,260 & 4,220 & & & \\
\hline \$95T003077 & \multirow[t]{2}{*}{$112: 11$} & Upper $1 / 2$ & 4,250 & 4,320 & 4,280 & & & \\
\hline S95T003078 & & Lower $1 / 2$ & 4,400 & 4,430 & 4,420 & & & \\
\hline S95T003079 & \multirow[t]{2}{*}{$112: 12$} & Upper $1 / 2$ & 3,710 & 3,780 & 3,740 & & & \\
\hline S95T003080 & & Lower $1 / 2$ & 4,210 & 4,190 & 4,200 & & & \\
\hline
\end{tabular}


Table B-11. Tank 241-B-204 Analytical Results: Chromium. (3 sheets)

\begin{tabular}{|c|c|c|c|c|c|c|c|c|}
\hline 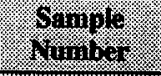 & $x_{0}^{*}$ & (5) & 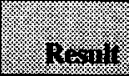 & 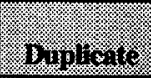 & 3 & (6) & 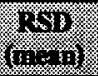 & 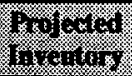 \\
\hline Hofig & & א. & 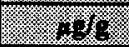 & 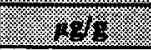 & 85 & 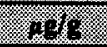 & 原 & (x) \\
\hline S95T003081 & \multirow[t]{2}{*}{$112: 13$} & Upper $1 / 2$ & 3,960 & 3,960 & 3,960 & & & \\
\hline S95T003082 & & Lower $1 / 2$ & 4,390 & 4,230 & 4,310 & & & \\
\hline S95T003083 & \multirow[t]{2}{*}{$112: 14$} & Upper $1 / 2$ & 3,250 & 3,370 & 3,310 & & & \\
\hline S95T003084 & & Lower $1 / 2$ & 1,940 & 1,860 & 1,900 & & & \\
\hline \$95T003282 & \multirow[t]{2}{*}{$114: 2$} & Upper $1 / 2$ & 1,370 & 1,300 & 1,340 & & & \\
\hline S95T003283 & & Lower $1 / 2$ & 1,840 & 1,940 & 1,890 & & & \\
\hline S95T003284 & \multirow[t]{2}{*}{$114: 3$} & Upper $1 / 2$ & 1,800 & 1,870 & 1,840 & & & \\
\hline S95T003285 & & Lower $1 / 2$ & 2,720 & 2,670 & 2,700 & & & \\
\hline S95T003286 & \multirow[t]{2}{*}{$114: 4$} & Upper $1 / 2$ & 1,970 & 1,920 & 1,940 & & & \\
\hline S95T003287 & & Lower $1 / 2$ & 2,460 & 2,510 & 2,480 & & & \\
\hline \$95T003288 & \multirow[t]{2}{*}{$114: 5$} & Upper $1 / 2$ & 2,470 & 2,320 & 2,400 & & & \\
\hline \$95T003289 & & Lower $1 / 2$ & 2,920 & 2,910 & 2,920 & & & \\
\hline S95T003290 & \multirow[t]{2}{*}{$114: 6$} & Upper $1 / 2$ & 3,100 & 2,820 & 2,960 & & & \\
\hline \$95T003291 & & Lower $1 / 2$ & 3,250 & 3,220 & 3,240 & & & \\
\hline S95T003292 & \multirow[t]{2}{*}{$114: 7$} & Upper $1 / 2$ & 4,130 & 4,320 & 4,220 & & & \\
\hline S95T003293 & & Lower $1 / 2$ & 3,590 & 3,250 & 3,420 & & & \\
\hline S95T003294 & \multirow[t]{2}{*}{$114: 8$} & Upper $1 / 2$ & 4,480 & 4,750 & 4,620 & & & \\
\hline S95T003295 & & Lower $1 / 2$ & 3,940 & 3,660 & 3,800 & & & \\
\hline S95T003296 & \multirow[t]{2}{*}{$114: 9$} & Upper $1 / 2$ & 4,100 & 3,910 & 4,000 & & & \\
\hline S95T003297 & & Lower $1 / 2$ & 4,470 & 4,360 & 4,420 & & & \\
\hline
\end{tabular}


Table B-11. Tank 241-B-204 Analytical Results: Chromium. (3 sheets)

\begin{tabular}{|c|c|c|c|c|c|c|c|c|}
\hline S Timples & $\begin{array}{l}\text { Eorrir } \\
\text { ceginer }\end{array}$ & Sognt. & Rerail & Barilione & (2) & $8.96+11$ & 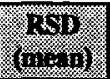 & 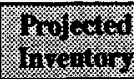 \\
\hline Eoblas: & \multirow{3}{*}{$114: 10$} & 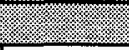 & 1. & (3) & 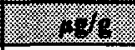 & 8.16 & 4 & 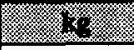 \\
\hline S95T003298 & & Upper $1 / 2$ & 3,550 & 3,300 & 3,420 & & & \\
\hline S95T003299 & & Lower $1 / 2$ & 3,980 & 4,020 & 4,000 & & & \\
\hline S95T003300 & \multirow[t]{2}{*}{$114: 11$} & Upper $1 / 2$ & 4,390 & 4,120 & 4,260 & & & \\
\hline S95T003301 & & Lower $1 / 2$ & 4,150 & 4,050 & 4,100 & & & \\
\hline S95T003302 & \multirow[t]{2}{*}{$114: 12$} & Upper $1 / 2$ & 3,220 & 3,390 & 3,300 & & & \\
\hline S95T003303 & & Lower $1 / 2$ & 3,890 & 3,930 & 3,910 & & & \\
\hline S95T003304 & \multirow[t]{2}{*}{$114: 13$} & Upper $1 / 2$ & 4,310 & 4,360 & 4,340 & & & \\
\hline S95T003305 & & Lower $1 / 2$ & 3,480 & 3,520 & 3,500 & & & \\
\hline S95T003306 & \multirow[t]{2}{*}{$114: 14$} & Upper $1 / 2$ & 2,250 & 2,230 & 2,240 & & & \\
\hline S95T003307 & & Lower $1 / 2$ & 1,680 & 1,780 & 1,730 & & & \\
\hline \multicolumn{3}{|c|}{ Drativible nifuids } & $1.1 \mathrm{ml}$ & 18fine & (19.1\% & Ojini. & (1) & 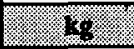 \\
\hline S95T002976 & $112: 1$ & DL & 154.1 & 152.0 & 153.0 & \multirow[t]{3}{*}{154} & \multirow[t]{3}{*}{2.7} & \multirow[t]{3}{*}{0.615} \\
\hline S95T002984 & $112: 2$ & $\mathrm{DL}$ & 146.0 & 146.0 & 146.0 & & & \\
\hline S95T003255 & 114:1 & DL & 161.0 & 155.0 & 158.0 & & & \\
\hline
\end{tabular}




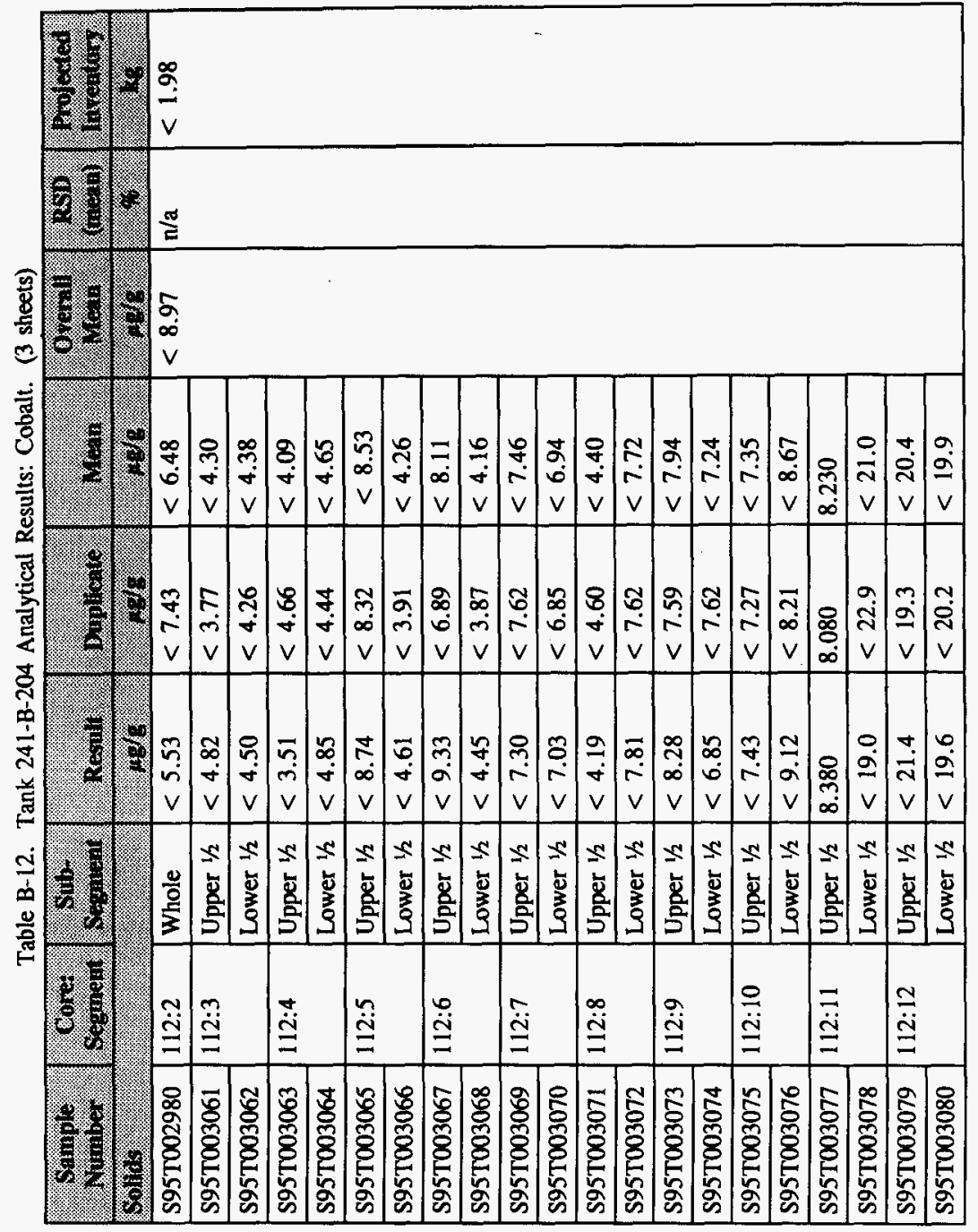


WHC-SD-WM-ER-581 Rev. 0

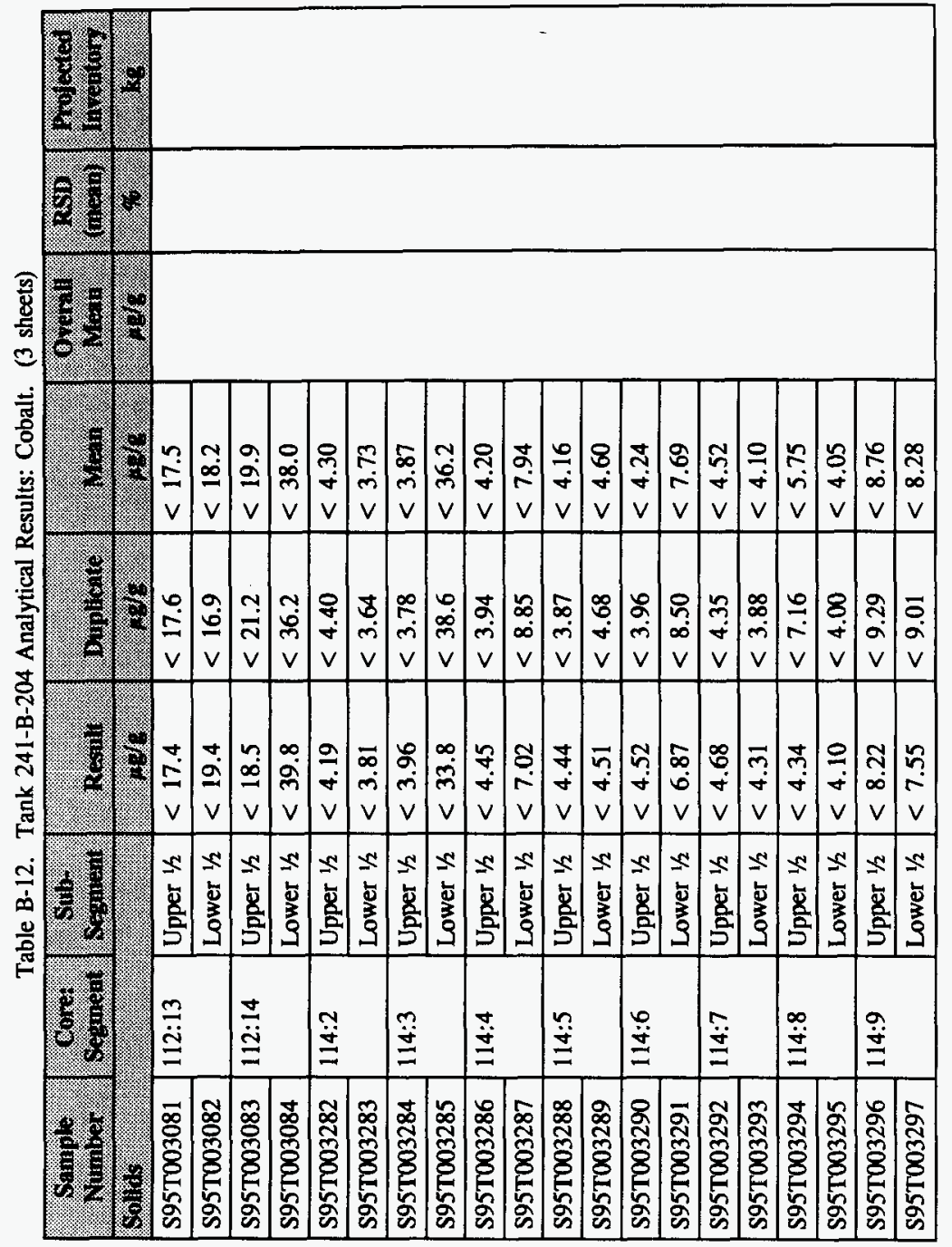


Table B-12. Tank 241-B-204 Analytical Results: Cobalt. (3 sheets)

\begin{tabular}{|c|c|c|c|c|c|c|c|c|}
\hline Sing & 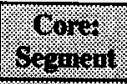 & (3) & (1) & BHDIIII) & 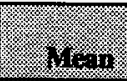 & $16 y+11$ & $\frac{1}{2 A B}$ & 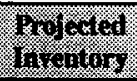 \\
\hline 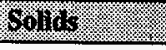 & \multirow{3}{*}{$114: 10$} & & 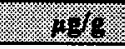 & (6) & 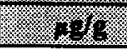 & 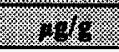 & 6. & (X) \\
\hline S95T003298 & & Upper $1 / 2$ & $<7.40$ & $<7.17$ & $<7.29$ & & & \\
\hline S95T003299 & & Lower $1 / 2$ & $<6.77$ & $<7.71$ & $<7.24$ & & & \\
\hline S95T003300 & \multirow[t]{2}{*}{$114: 11$} & Upper $1 / 2$ & $<9.40$ & $<9.66$ & $<9.53$ & & & \\
\hline S95T003301 & & Lower $1 / 2$ & $<6.80$ & $<6.82$ & $<6.81$ & & & \\
\hline S95T003302 & \multirow[t]{2}{*}{ 114:12 } & Upper $1 / 2$ & $<4.80$ & $<4.42$ & $<4.61$ & & & \\
\hline S95T003303 & & Lower $1 / 2$ & $<4.47$ & $<7.41$ & $<5.94$ & & & \\
\hline S95T003304 & \multirow[t]{2}{*}{$114: 13$} & Upper $1 / 2$ & $<9.16$ & $<8.18$ & $<8.67$ & & & \\
\hline S95T003305 & & Lower $1 / 2$ & $<7.21$ & $<7.31$ & $<7.26$ & & & \\
\hline S95T003306 & \multirow[t]{2}{*}{$114: 14$} & Upper $1 / 2$ & $<8.58$ & $<8.55$ & $<8.57$ & & & \\
\hline S95T003307 & & Lower $1 / 2$ & $<7.73$ & $<8.46$ & $<8.10$ & & & \\
\hline \multicolumn{3}{|c|}{ Wranate lauids } & aglin & $\sqrt{4 \sin 8}$ & 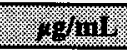 & $14 /$ inis & . & 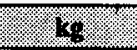 \\
\hline S95T002976 & $112: 1$ & DL & $<4.02$ & $<4.02$ & $<4.02$ & \multirow[t]{3}{*}{$<3.02$} & \multirow[t]{3}{*}{$n / a$} & \multirow[t]{3}{*}{$<0.0121$} \\
\hline \$95T002984 & $112: 2$ & DL & $<4.02$ & $<4.02$ & $<4.02$ & & & \\
\hline S95T003255 & $114: 1$ & $\overline{\mathrm{DL}}$ & $<2.02$ & $<2.02$ & $<2.02$ & & & \\
\hline
\end{tabular}


Table B-13. Tank 241-B-204 Analytical Results: Copper. (3 sheets)

\begin{tabular}{|c|c|c|c|c|c|c|c|c|}
\hline 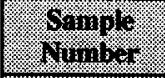 & \%or & oil1: & Silenili & 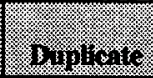 & ly & 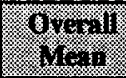 & 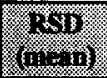 & 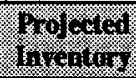 \\
\hline $2 \% 11 \%$ & & 8 & (1) & 80 & 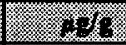 & 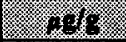 & . & $\frac{1}{4}$ \\
\hline S95T002980 & $112: 2$ & Whole & 39.20 & 11.30 & 25.25 & \multirow[t]{21}{*}{22.1} & \multirow[t]{21}{*}{80.4} & \multirow[t]{21}{*}{4.87} \\
\hline S95T003061 & \multirow[t]{2}{*}{$112: 3$} & Upper $1 / 2$ & 3.190 & $<1.88$ & 2.54 & & & \\
\hline S95T003062 & & Lower $1 / 2$ & 12.20 & 9.760 & 10.98 & & & \\
\hline S95T003063 & \multirow[t]{2}{*}{$112: 4$} & Upper $1 / 2$ & 2.740 & 3.580 & 3.160 & & & \\
\hline S95T003064 & & Lower $1 / 2$ & 92.60 & 90.30 & 91.45 & & & \\
\hline S95T003065 & \multirow[t]{2}{*}{$112: 5$} & Upper $1 / 2$ & 20.50 & 15.30 & 17.90 & & & \\
\hline S95T003066 & & Lower $1 / 2$ & 4.880 & 2.720 & 3.800 & & & \\
\hline S95T003067 & \multirow[t]{2}{*}{$112: 6$} & Upper $1 / 2$ & 4.970 & $<3.46$ & 4.22 & & & \\
\hline \$95T003068 & & Lower $1 / 2$ & 2.290 & $<1.94$ & 2.12 & & & \\
\hline S95T003069 & \multirow[t]{2}{*}{$112: 7$} & Upper $1 / 2$ & $<3.64$ & $<3.81$ & $<3.73$ & & & \\
\hline S95T003070 & & Lower $1 / 2$ & $<3.51$ & $<3.43$ & $<3.47$ & & & \\
\hline S95T003071 & \multirow[t]{2}{*}{$112: 8$} & Upper $1 / 2$ & $<2.10$ & $<2.30$ & $<2.20$ & & & \\
\hline$\$ 95 T 003072$ & & Lower $1 / 2$ & 4.460 & $<3.81$ & 4.14 & & & \\
\hline S95T003073 & \multirow[t]{2}{*}{$112: 9$} & Upper $1 / 2$ & 5.170 & 4.300 & 4.735 & & & \\
\hline S95T003074 & & Lower $1 / 2$ & $<3.43$ & $<3.81$ & $<3.62$ & & & \\
\hline S95T003075 & \multirow[t]{2}{*}{$112: 10$} & Upper $1 / 2$ & 3.720 & 7.810 & 5.767 & & & \\
\hline S95T003076 & & Lower $1 / 2$ & 7.220 & 5.940 & 6.580 & & & \\
\hline S95T003077 & \multirow[t]{2}{*}{ 112:11 } & Upper $1 / 2$ & 9.410 & 8.690 & 9.050 & & & \\
\hline S95T003078 & & Lower $1 / 2$ & $<9.49$ & $<11.5$ & $<10.5$ & & & \\
\hline S95T003079 & \multirow[t]{2}{*}{$112: 12$} & Upper $1 / 2$ & $<10.7$ & $<9.66$ & $<10.2$ & & & \\
\hline S95T003080 & & Lower $1 / 2$ & 19.70 & 1,450 & 734.9 & & & \\
\hline
\end{tabular}


Table B-13. Tank 241-B-204 Analytical Results: Copper. (3 sheets)

\begin{tabular}{|c|c|c|c|c|c|c|c|c|}
\hline Malieps & 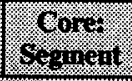 & 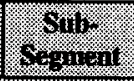 & Resnin & $b_{1131}, 410$ & 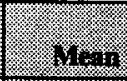 & $64 x+110$ & (110) & 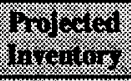 \\
\hline Soling & 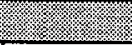 & & 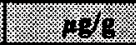 & 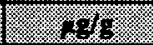 & 25 & 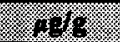 & \% & (3) \\
\hline S95T003081 & \multirow[t]{2}{*}{$112: 13$} & Upper $1 / 2$ & $<8.72$ & $<8.73$ & $<8.73$ & & & \multirow{20}{*}{, } \\
\hline S95T003082 & & Lower $1 / 2$ & $<9.72$ & $<8.47$ & $<9.10$ & & & \\
\hline S95T003083 & \multirow[t]{2}{*}{ 112:14 } & Upper $1 / 2$ & $<9.26$ & $<10.6$ & $<9.93$ & & & \\
\hline S95T003084 & & Lower $1 / 2$ & $<19.9$ & $<18.1$ & $<19.0$ & & & \\
\hline S95T003282 & \multirow[t]{2}{*}{$114: 2$} & Upper $1 / 2$ & $<2.10$ & $<2.20$ & $<2.15$ & & & \\
\hline S95T003283 & & Lower $1 / 2$ & $<1.95$ & $<1.82$ & $<1.89$ & & & \\
\hline S95T003284 & \multirow[t]{2}{*}{$114: 3$} & Upper $1 / 2$ & 9.350 & 8.270 & 8.810 & & & \\
\hline S95T003285 & & Lower $1 / 2$ & $<16.9$ & $<19.3$ & $<18.1$ & & & \\
\hline S95T003286 & \multirow[t]{2}{*}{$114: 4$} & Upper $1 / 2$ & 4.070 & 3.500 & 3.785 & & & \\
\hline S95T003287 & & Lower $1 / 2$ & $<3.51$ & $<4.43$ & $<3.97$ & & & \\
\hline S95T003288 & \multirow[t]{2}{*}{$114: 5$} & Upper $1 / 2$ & 4.170 & 2.130 & 3.150 & & & \\
\hline S95T003289 & & Lower $1 / 2$ & 3.010 & 4.590 & 3.800 & & & \\
\hline S95T003290 & \multirow[t]{2}{*}{$114: 6$} & Upper $1 / 2$ & 4.020 & $<1.98$ & 3.00 & & & \\
\hline S95T003291 & & Lower $1 / 2$ & $<3.44$ & $<4.25$ & $<3.85$ & & & \\
\hline S95T003292 & \multirow[t]{2}{*}{$114: 7$} & Upper $1 / 2$ & 2.590 & $<2.17$ & 2.38 & & & \\
\hline S95T003293 & & Lower $1 / 2$ & $<2.16$ & 2.790 & 2.48 & & & \\
\hline S95T003294 & \multirow[t]{2}{*}{$114: 8$} & Upper $1 / 2$ & $<2.17$ & $<3.58$ & $<2.88$ & & & \\
\hline S95T003295 & & Lower $1 / 2$ & $<2.05$ & 2.800 & 2.43 & & & \\
\hline S95T003296 & \multirow[t]{2}{*}{$114: 9$} & Upper $1 / 2$ & $<4.11$ & $<4.64$ & $<4.38$ & & & \\
\hline S95T003297 & & Lower $1 / 2$ & $<3.79$ & 5.010 & 4.40 & & & \\
\hline
\end{tabular}


Table B-13. Tank 241-B-204 Analytical Results: Copper. (3 sheets)

\begin{tabular}{|c|c|c|c|c|c|c|c|c|}
\hline Minplo & 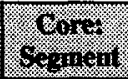 & 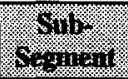 & ingin & $(3,1])=210$ & $91 \%$ & 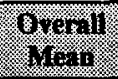 & 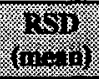 & 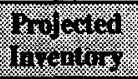 \\
\hline Solits & י. & (2) & (3) & $3 \%$ & 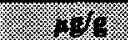 & 40 & ; & 8 \\
\hline S95T003298 & \multirow[t]{2}{*}{$114: 10$} & Upper $1 / 2$ & $<3.70$ & 3.700 & 3.70 & & & \\
\hline S95T003299 & & Lower $1 / 2$ & 5.050 & 7.570 & 6.310 & & & \\
\hline S95T003300 & \multirow[t]{2}{*}{$114: 11$} & Upper $1 / 2$ & 7.230 & 9.090 & 8.160 & & & \\
\hline S95T003301 & & Lower $1 / 2$ & 4.560 & 5.930 & 5.245 & & & \\
\hline S95T003302 & \multirow[t]{2}{*}{$114: 12$} & Upper $1 / 2$ & 2.850 & 4.530 & 3.690 & & & \\
\hline S95T003303 & & Lower $1 / 2$ & 3.210 & $<3.70$ & 3.46 & & & \\
\hline S95T003304 & \multirow[t]{2}{*}{$114: 13$} & Upper $1 / 2$ & $<4.58$ & $<4.09$ & $<4.34$ & & & \\
\hline S95T003305 & & Lower $1 / 2$ & $<3.60$ & $<3.66$ & $<3.63$ & & & \\
\hline S95T003306 & \multirow[t]{2}{*}{$114: 14$} & Upper $1 / 2$ & 5.150 & $<4.27$ & 4.71 & & & \\
\hline S95T003307 & & Lower $1 / 2$ & $<3.86$ & 4.510 & 4.19 & & & \\
\hline \multicolumn{3}{|c|}{ Bralinble liguts } & $49 / 111$ & $4 / m i$ & Q/m & (IIII) & (6) & 18 \\
\hline S95T002976 & 112:1 & DL & $<2.01$ & $<2.01$ & $<2.01$ & \multirow[t]{3}{*}{$<1.51$} & \multirow[t]{3}{*}{$n / 2$} & \multirow[t]{3}{*}{$<0.00604$} \\
\hline S95T002984 & $112: 2$ & DL & $<2.01$ & $<2.01$ & $<2.01$ & & & \\
\hline S95T003255 & $114: 1$ & DL & $<1.01$ & $<1.01$ & $<1.01$ & & & \\
\hline
\end{tabular}




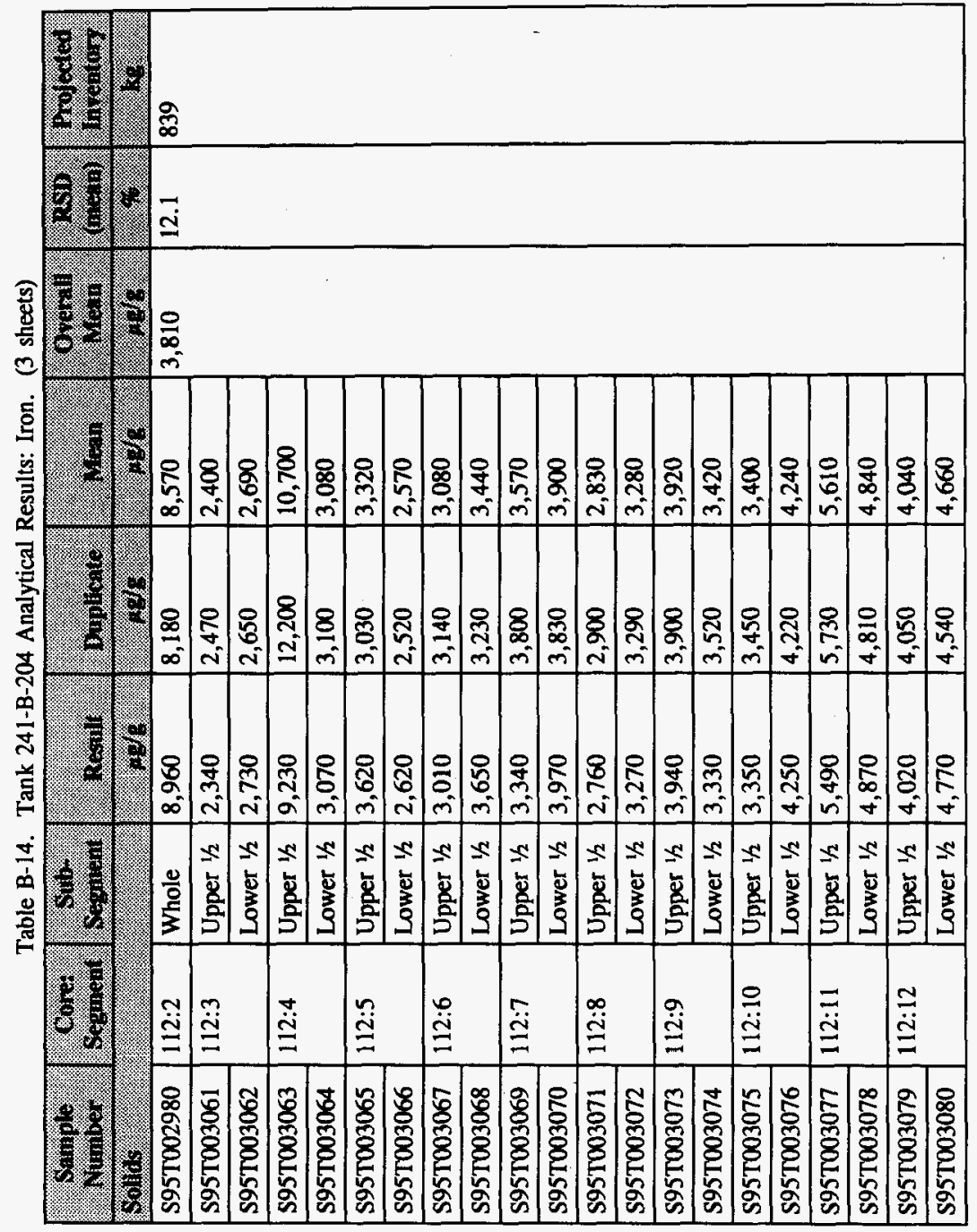




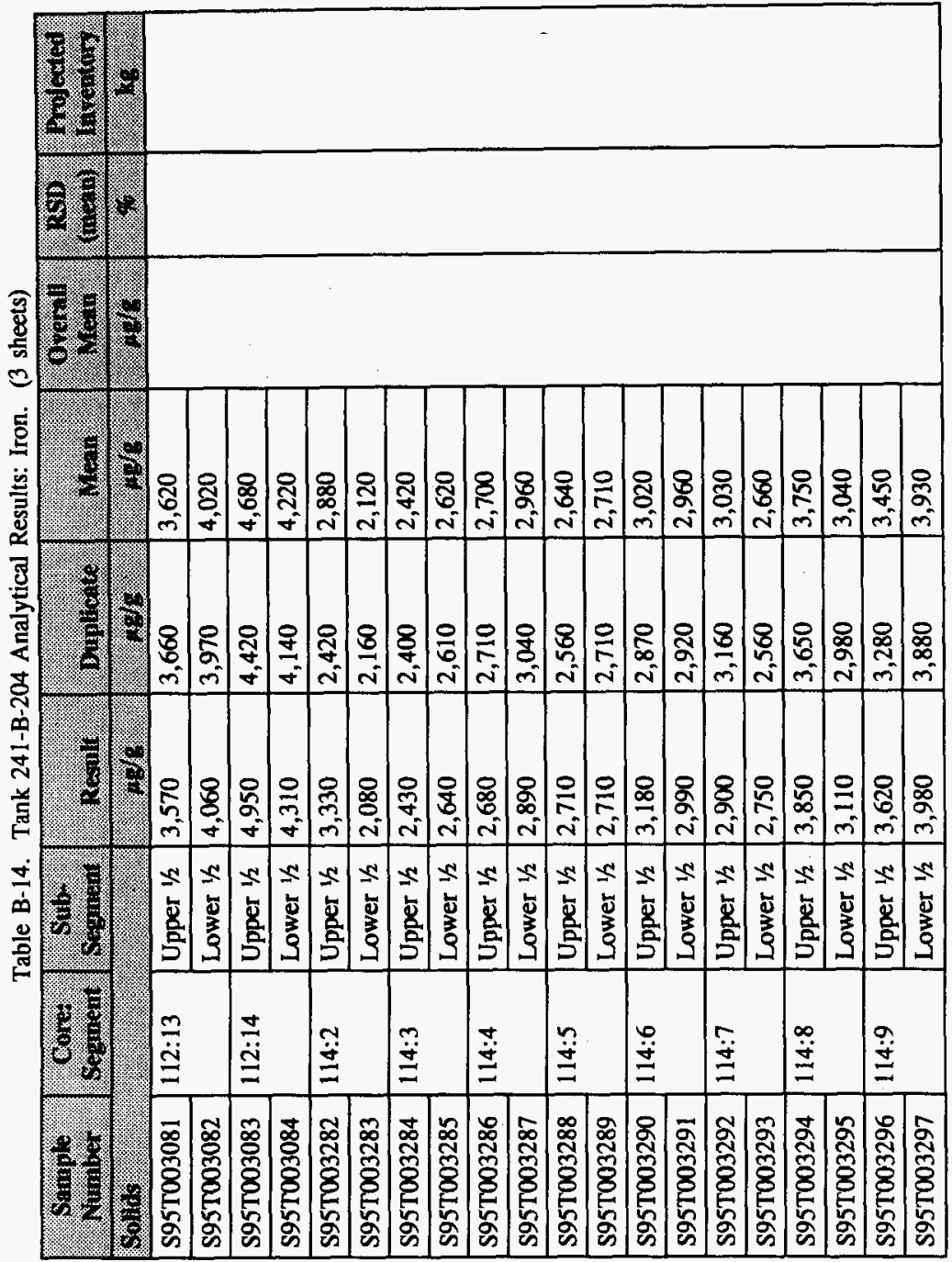


Table B-14. Tank 241-B-204 Analytical Results: Iron. (3 sheets)

\begin{tabular}{|c|c|c|c|c|c|c|c|c|}
\hline 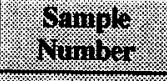 & 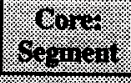 & 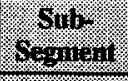 & pe.ilil & 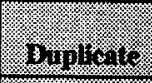 & 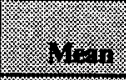 & Wy & 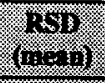 & 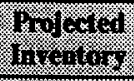 \\
\hline Sollis & & = & , & (1) & 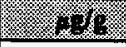 & 1. & (x) & (4) \\
\hline S95T003298 & \multirow[t]{2}{*}{$114: 10$} & Upper $1 / 2$ & 3,730 & 3,500 & 3,620 & & & \\
\hline S95T003299 & & Lower $1 / 2$ & 3,810 & 3,830 & 3,820 & & & \\
\hline \$95T003300 & \multirow[t]{2}{*}{$114: 11$} & Upper $1 / 2$ & 5,590 & 5,350 & 5,470 & & & \\
\hline S95T003301 & & Lower $1 / 2$ & 4,150 & 4,080 & 4,120 & & & \\
\hline \$95T003302 & \multirow[t]{2}{*}{$114: 12$} & Upper $1 / 2$ & 3,150 & 3,350 & 3,250 & & & \\
\hline S95T003303 & & Lower $1 / 2$ & 3,780 & 3,820 & 3,800 & & & \\
\hline S95T003304 & \multirow[t]{2}{*}{$114: 13$} & Upper $1 / 2$ & 3,850 & 3,850 & 3,850 & & & \\
\hline S95T003305 & & Lower $1 / 2$ & 3,380 & 3,430 & 3,400 & & & \\
\hline S95T003306 & \multirow[t]{2}{*}{$114: 14$} & Upper $1 / 2$ & 3,640 & 3,610 & 3,620 & & & \\
\hline S95T003307 & & Lower $1 / 2$ & 3,590 & 3,870 & 3,730 & & & \\
\hline \multicolumn{2}{|c|}{ Bualing ble rigulas } & & 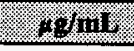 & (1) & . & 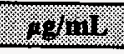 & 13 & 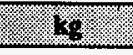 \\
\hline S95T002976 & 112:1 & $\mathrm{DL}$ & $<10.0$ & $<10.0$ & $<10.0$ & \multirow[t]{3}{*}{$<7.53$} & \multirow[t]{3}{*}{$n / a$} & \multirow[t]{3}{*}{$<0.0301$} \\
\hline S95T002984 & $112: 2$ & $\mathrm{DL}$ & $<10.0$ & $<10.0$ & $<10.0$ & & & \\
\hline S95T003255 & $114: 1$ & $\mathrm{DL}$ & $<5.05$ & $<5.05$ & $<5.05$ & & & \\
\hline
\end{tabular}


Table B-15. Tank 241-B-204 Analytical Results: Lanthanum. (3 sheets)

\begin{tabular}{|c|c|c|c|c|c|c|c|c|}
\hline Minger & (6) & ( & Panil & Binglionge & Mrin & hyong & $\frac{1518}{(100111}$ & 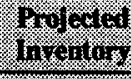 \\
\hline $801 \%$ & (1) & (1). & 18 & I 19 & (2) & (1) & 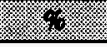 & 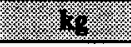 \\
\hline S95T002980 & $112: 2$ & Whole & 8,170 & 7,560 & 7,860 & \multirow[t]{21}{*}{10,400} & \multirow[t]{21}{*}{2.6} & \multirow[t]{21}{*}{2,290} \\
\hline$\$ 95 T 003061$ & \multirow[t]{2}{*}{$112: 3$} & Upper $1 / 2$ & 8,890 & 9,380 & 9,140 & & & \\
\hline S95T003062 & & Lower $1 / 2$ & 10,500 & 10,300 & 10,400 & & & \\
\hline S95T003063 & \multirow[t]{2}{*}{$112: 4$} & Upper $1 / 2$ & 8,910 & 11,700 & 10,300 & & & \\
\hline S95T003064 & & Lower $1 / 2$ & 11,400 & 11,400 & 11,400 & & & \\
\hline S95T003065 & \multirow[t]{2}{*}{$112: 5$} & Upper $1 / 2$ & 10,900 & 9,040 & 9,970 & & & \\
\hline S95T003066 & & Lower $1 / 2$ & 9,130 & 8,710 & 8,920 & & & \\
\hline \$95T003067 & \multirow[t]{2}{*}{$112: 6$} & Upper $1 / 2$ & 11,000 & 11,800 & 11,400 & & & \\
\hline S95T003068 & & Lower $1 / 2$ & 10,200 & 8,950 & 9,580 & & & \\
\hline \$95T003069 & \multirow[t]{2}{*}{$112: 7$} & Upper $1 / 2$ & 10,300 & 11,200 & 10,800 & & & \\
\hline S95T003070 & & Lower $1 / 2$ & 12,700 & 12,000 & 12,400 & & & \\
\hline S95T003071 & \multirow[t]{2}{*}{$112: 8$} & Upper $1 / 2$ & 7,730 & 8,370 & 8,050 & & & \\
\hline S95T003072 & & Lower $1 / 2$ & 8,430 & 8,330 & 8,380 & & & \\
\hline S95T003073 & \multirow[t]{2}{*}{$112: 9$} & Upper $1 / 2$ & 10,100 & 9,940 & 10,000 & & & \\
\hline S95T003074 & & Lower $1 / 2$ & 9,870 & 9,930 & 9,900 & & & \\
\hline S95T003075 & \multirow[t]{2}{*}{$112: 10$} & Upper $1 / 2$ & 9,280 & 9,810 & 9,540 & & & \\
\hline S95T003076 & & Lower $1 / 2$ & 9,300 & 10,400 & 9,850 & & & \\
\hline S95T003077 & \multirow[t]{2}{*}{$112: 11$} & Upper $1 / 2$ & 8,430 & 9,370 & 8,900 & & & \\
\hline S95T003078 & & Lower $1 / 2$ & 10,900 & 9,950 & 10,400 & & & \\
\hline S95T003079 & \multirow[t]{2}{*}{$112: 12$} & Upper $1 / 2$ & 11,500 & 10,600 & 11,000 & & & \\
\hline S95T003080 & & Lower $1 / 2$ & 14,700 & 11,700 & 13,200 & & & \\
\hline
\end{tabular}




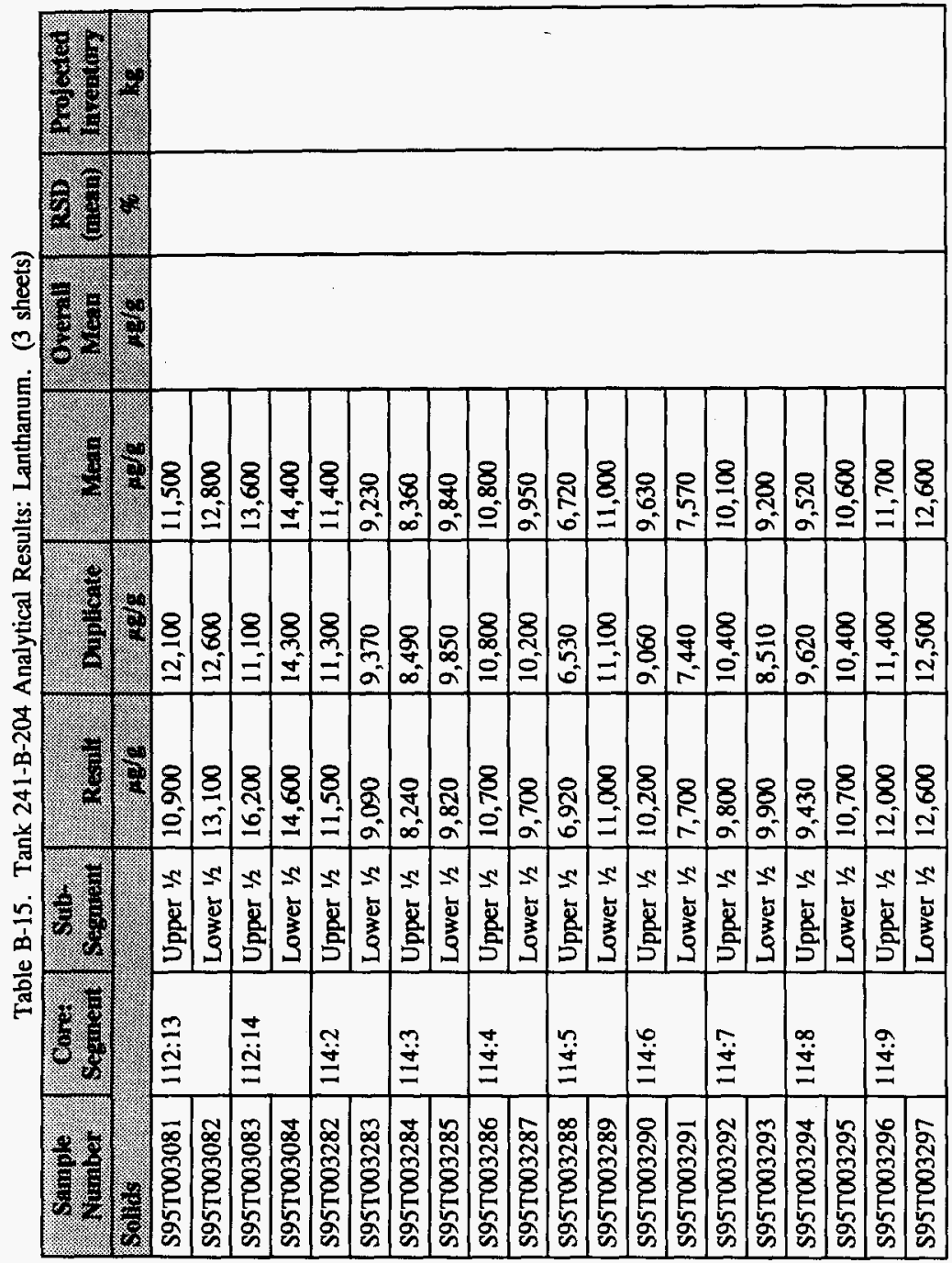


Table B-15. Tank 241-B-204 Analytical Results: Lanthanum. (3 sheets)

\begin{tabular}{|c|c|c|c|c|c|c|c|c|}
\hline . & 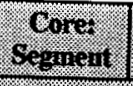 & 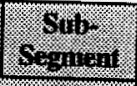 & resiln & 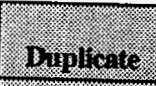 & 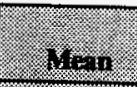 & 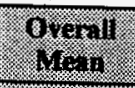 & 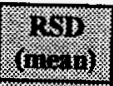 & 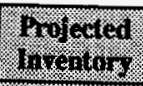 \\
\hline 6011,5 & 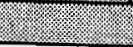 & (r) & Pf & 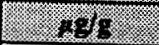 & 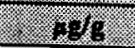 & 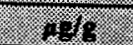 & 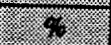 & 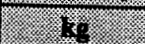 \\
\hline S95T003298 & \multirow[t]{2}{*}{$114: 10$} & Upper $1 / 2$ & 10,600 & 10,100 & 10,400 & & & \\
\hline S95T003299 & & Lower $1 / 2$ & 9,600 & 9,830 & 9,720 & & & \\
\hline S95T003300 & \multirow[t]{2}{*}{$114: 11$} & Upper $1 / 2$ & 10,900 & 10,100 & 10,500 & & & \\
\hline S95T003301 & & Lower $1 / 2$ & 10,600 & 10,300 & 10,400 & & & \\
\hline \$95T003302 & \multirow[t]{2}{*}{$114: 12$} & Upper $1 / 2$ & 8,850 & 9,700 & 9,280 & & & \\
\hline S95T003303 & & Lower $1 / 2$ & 11,400 & 11,500 & 11,400 & & & \\
\hline S95T003304 & \multirow[t]{2}{*}{$114: 13$} & Upper $1 / 2$ & 13,200 & 14,000 & 13,600 & & & \\
\hline S95T003305 & & Lower $1 / 2$ & 10,600 & 10,100 & 10,400 & & & \\
\hline S95T003306 & \multirow[t]{2}{*}{$114: 14$} & Upper $1 / 2$ & 11,500 & 11,600 & 11,600 & & & \\
\hline \$95T003307 & & Lower $1 / 2$ & 12,000 & 12,800 & 12,400 & & & \\
\hline \multicolumn{3}{|c|}{ Drainable liqulos } & apditis & $41 \mathrm{mi}$ & 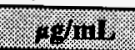 & 211113 & 2. & \% \\
\hline$\$ 95 T 002976$ & $112: 1$ & DL & $<10.0$ & $<10.0$ & $<10.0$ & \multirow[t]{3}{*}{$<7.53$} & \multirow[t]{3}{*}{$n / a$} & \multirow[t]{3}{*}{$<0.0301$} \\
\hline \$95T002984 & $112: 2$ & $\mathrm{DL}$ & $<10.0$ & $<10.0$ & $<10.0$ & & & \\
\hline S95T003255 & $114: 1$ & $\mathrm{DL}$ & $<5.05$ & $<5.05$ & $<5.05$ & & & \\
\hline
\end{tabular}


Table B-16. Tank 241-B-204 Analytical Results: Lead. (3 sheets)

\begin{tabular}{|c|c|c|c|c|c|c|c|c|}
\hline 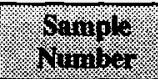 & 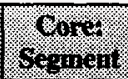 & 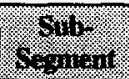 & towin & BHol) & 18 & 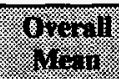 & yin & 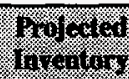 \\
\hline 60115 & & 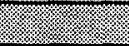 & $30 / 6$ & 48 & 40 & .16 & 9.6 & 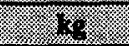 \\
\hline \$95T002980 & $112: 2$ & Whole & 610.0 & 568.0 & 589.0 & \multirow[t]{21}{*}{$<1,070$} & \multirow[t]{21}{*}{$n / a$} & \multirow[t]{21}{*}{$<236$} \\
\hline \$95T003061 & \multirow[t]{2}{*}{$112: 3$} & Upper $1 / 2$ & $<24.1$ & $<18.8$ & $<21.5$ & & & \\
\hline S95T003062 & & Lower $1 / 2$ & $<22.5$ & $<21.3$ & $<21.9$ & & & \\
\hline S95T003063 & \multirow[t]{2}{*}{$112: 4$} & Upper $1 / 2$ & $<17.6$ & $<23.3$ & $<20.5$ & & & \\
\hline S95T003064 & & Lower $1 / 2$ & 38.60 & 44.30 & 41.45 & & & \\
\hline S95T003065 & \multirow[t]{2}{*}{$112: 5$} & Upper $1 / 2$ & $<43.7$ & $<41.6$ & $<42.7$ & & & \\
\hline S95T003066 & & Lower $1 / 2$ & $<23.1$ & 19.60 & 21.4 & & & \\
\hline S95T003067 & \multirow[t]{2}{*}{$112: 6$} & Upper $1 / 2$ & $<46.6$ & $<34.6$ & $<40.6$ & & & \\
\hline S95T003068 & & Lower $1 / 2$ & 30.30 & 27.40 & 28.85 & & & \\
\hline S95T003069 & \multirow[t]{2}{*}{$112: 7$} & Upper $1 / 2$ & 47.10 & 40.90 & 44.0 & & & \\
\hline S95T003070 & & Lower $1 / 2$ & $<35.1$ & $<34.3$ & $<34.7$ & & & \\
\hline S95T003071 & \multirow[t]{2}{*}{$112: 8$} & Upper $1 / 2$ & $<21.0$ & $<23.0$ & $<22.0$ & & & \\
\hline S95T003072 & & Lower $1 / 2$ & 357.0 & 366.0 & 361.5 & & & \\
\hline S95T003073 & \multirow[t]{2}{*}{$112: 9$} & Upper $1 / 2$ & 557.0 & 557.0 & 557.0 & & & \\
\hline S95T003074 & & Lower $1 / 2$ & 485.0 & 580.0 & 532.5 & & & \\
\hline S95T003075 & \multirow[t]{2}{*}{$112: 10$} & Upper $1 / 2$ & 39,400 & $40, \overline{300}$ & 39,800 & & & \\
\hline \$95T003076 & & Lower $1 / 2$ & 103.0 & 94.40 & 98.70 & & & \\
\hline S95T003077 & \multirow[t]{2}{*}{$112: 11$} & Upper $1 / 2$ & $<40.7$ & $<34.8$ & $<37.8$ & & & \\
\hline S95T003078 & & Lower $1 / 2$ & $<94.9$ & $<115$ & $<105$ & & & \\
\hline S95T003079 & \multirow[t]{2}{*}{$112: 12$} & Upper $1 / 2$ & $<107$ & $<96.6$ & $<102$ & & & \\
\hline S95T003080 & & Lower $1 / 2$ & $<97.8$ & $<101$ & $<\overline{99.4}$ & & & \\
\hline
\end{tabular}


Table B-16. Tank 241-B-204 Analytical Results: Lead. (3 sheets)

\begin{tabular}{|c|c|c|c|c|c|c|c|c|}
\hline 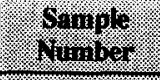 & 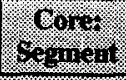 & (1) & Resuil & 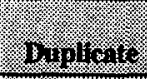 & $4=$ & 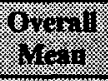 & $\begin{array}{c}353 \\
31+11\end{array}$ & $\frac{p}{604}$ \\
\hline 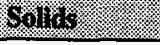 & & & $8 y^{3}$ & 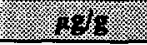 & 30) & 18 & , & 25. \\
\hline S95T003081 & \multirow[t]{2}{*}{$112: 13$} & Upper $1 / 2$ & $<87.2$ & $<87.3$ & $<87.3$ & & & \\
\hline S95T003082 & & Lower $1 / 2$ & $<97.2$ & $<84.7$ & $<91.0$ & & & \\
\hline S95T003083 & \multirow[t]{2}{*}{$112: 14$} & Upper $1 / 2$ & 1,310 & 825.0 & 1070 & & & \\
\hline S95T003084 & & Lower $1 / 2$ & 3,780 & 3,710 & 3,740 & & & \\
\hline \$95T003282 & \multirow[t]{2}{*}{$114: 2$} & Upper $1 / 2$ & $<21.0$ & $<22.0$ & $<21.5$ & & & \\
\hline \$95T003283 & & Lower $1 / 2$ & $<19.5$ & $<18.2$ & $<18.9$ & & & \\
\hline$\$ 957003284$ & \multirow[t]{2}{*}{$114: 3$} & Upper $1 / 2$ & $<19.8$ & $<18.9$ & $<19.35$ & & & \\
\hline S95T003285 & & Lower $1 / 2$ & $<169$ & $<193$ & $<181$ & & & \\
\hline S95T003286 & \multirow[t]{2}{*}{$114: 4$} & Upper $1 / 2$ & $<22.3$ & $<19.7$ & $<21.0$ & & & \\
\hline S95T003287 & & Lower $1 / 2$ & $<35.1$ & $<44.3$ & $<39.7$ & & & \\
\hline S95T003288 & \multirow[t]{2}{*}{$114: 5$} & Upper $1 / 2$ & $<22.2$ & $<19.3$ & $<20.8$ & & & \\
\hline S95T003289 & & Lower $1 / 2$ & $<22.6$ & $<23.4$ & $<23.0$ & & & \\
\hline S95T003290 & \multirow[t]{2}{*}{$114: 6$} & Upper $1 / 2$ & $<22.6$ & $<19.8$ & $<21.2$ & & & \\
\hline S95T003291 & & Lower $1 / 2$ & $<34.4$ & $<42.5$ & $<38.5$ & & & \\
\hline S95T003292 & \multirow[t]{2}{*}{$114: 7$} & Upper $1 / 2$ & $<23.4$ & $<21.7$ & $<22.6$ & & & \\
\hline S95T003293 & & Lower $1 / 2$ & $<21.6$ & $<19.4$ & $<20.5$ & & & \\
\hline S95T003294 & \multirow[t]{2}{*}{$114: 8$} & Upper $1 / 2$ & $<21.7$ & $<35.8$ & $<28.8$ & & & \\
\hline S95T003295 & & Lower $1 / 2$ & $<20.5$ & $<20.0$ & $<20.3$ & & & \\
\hline S95T003296 & \multirow[t]{2}{*}{$114: 9$} & Upper $1 / 2$ & $<41.1$ & $<46.4$ & $<43.7$ & & & \\
\hline S95T003297 & & Lower $1 / 2$ & $<37.9$ & $<45.0$ & $<41.5$ & & & \\
\hline
\end{tabular}


Table B-16. Tank 241-B-204 Analytical Results: Lead. (3 sheets)

\begin{tabular}{|c|c|c|c|c|c|c|c|c|}
\hline Sample & 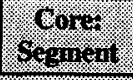 & (6) & Iteniti: & Bispisite & 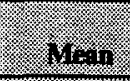 & 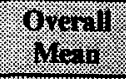 & 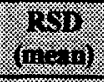 & 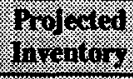 \\
\hline Som & : & 盾 & . $2 \% 6$ & 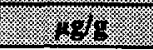 & 28 & $(x)=$ & . & (1) \\
\hline S95T003298 & \multirow[t]{2}{*}{$114: 10$} & Upper $1 / 2$ & $<37.0$ & $<35.9$ & $<36.5$ & & & \\
\hline \$95T003299 & & Lower $1 / 2$ & $<33.9$ & $<38.5$ & $<36.2$ & & & \\
\hline S95T003300 & \multirow[t]{2}{*}{$114: 11$} & Upper $1 / 2$ & $<47.0$ & $<48.3$ & $<47.7$ & & & \\
\hline S95T003301 & & Lower $1 / 2$ & $<34.0$ & $<34.0$ & $<34.0$ & & & \\
\hline S95T003302 & \multirow[t]{2}{*}{$114: 12$} & Upper $1 / 2$ & $<24.0$ & $<22.1$ & $<23.1$ & & & \\
\hline S95T003303 & & Lower $1 / 2$ & $<22.3$ & $<37.0$ & $<29.7$ & & & \\
\hline S95T003304 & \multirow[t]{2}{*}{ 114:13 } & Upper $1 / 2$ & $<45.8$ & $<40.9$ & $<43.4$ & & & \\
\hline \$95T003305 & & Lower $1 / 2$ & 40.40 & 50.50 & 45.45 & & & \\
\hline S95T003306 & \multirow[t]{2}{*}{$114: 14$} & Upper $1 / 2$ & 1,470 & 1,460 & 1,460 & & & \\
\hline S95T003307 & & Lower $1 / 2$ & 5,140 & 5,460 & 5,300 & & & \\
\hline \multicolumn{3}{|c|}{ Brimible linulds } & (4) grint & $13 / \min$ & 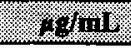 & $49 i 11$. & . & 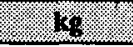 \\
\hline S95T002976 & 112:1 & DL & $<20.1$ & $<20.1$ & $<20.1$ & \multirow[t]{3}{*}{$<15.1$} & \multirow[t]{3}{*}{$n / a$} & \multirow[t]{3}{*}{$<0.0604$} \\
\hline S95T002984 & $112: 2$ & DL & $<20.1$ & $<20.1$ & $<20.1$ & & & \\
\hline S95T003255 & $114: 1$ & $\overline{D L}$ & $<10.1$ & $<10.1$ & $<10.1$ & & & \\
\hline
\end{tabular}


Table B-17. Tank 241-B-204 Analytical Results: Magnesium. (3 sheets)

\begin{tabular}{|c|c|c|c|c|c|c|c|c|}
\hline Gompls & 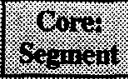 & $\frac{10}{611}$ & \% & 1401) gate & $(1 / 2+4$ & ofyen & $\frac{1413}{(1 ; 1)}$ & Work \\
\hline Solits & & א. & 28 & 1.96 & 28 & (1) & 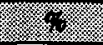 & 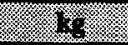 \\
\hline \$95T002980 & $112: 2$ & Whole & 356.0 & 322.0 & 339.0 & \multirow[t]{21}{*}{82.8} & \multirow[t]{21}{*}{20.2} & \multirow[t]{21}{*}{18.2} \\
\hline S95T003061 & \multirow[t]{2}{*}{$112: 3$} & Upper $1 / 2$ & 62.10 & 69.60 & 65.85 & & & \\
\hline S95T003062 & & Lower $1 / 2$ & 88.20 & 83.10 & 85.65 & & & \\
\hline S95T003063 & \multirow[t]{2}{*}{$112: 4$} & Upper $1 / 2$ & 61.60 & 80.60 & 71.10 & & & \\
\hline S95T003064 & & Lower $1 / 2$ & 72.10 & 76.20 & 74.15 & & & \\
\hline S95T003065 & \multirow[t]{2}{*}{$112: 5$} & Upper $1 / 2$ & 71.70 & 54.30 & 63.00 & & & \\
\hline \$95T003066 & & Lower $1 / 2$ & 50.70 & 50.30 & 50.50 & & & \\
\hline S95T003067 & \multirow[t]{2}{*}{$112: 6$} & Upper $1 / 2$ & 55.50 & 43.10 & 49.30 & & & \\
\hline S95T003068 & & Lower $1 / 2$ & 59.20 & 53.00 & 56.10 & & & \\
\hline$\$ 95 T 003069$ & \multirow[t]{2}{*}{$112: 7$} & Upper $1 / 2$ & $<36.4$ & 50.60 & 43.5 & & & \\
\hline S95T003070 & & Lower $1 / 2$ & 45.20 & 48.20 & 46.70 & & & \\
\hline S95T003071 & \multirow[t]{2}{*}{$112: 8$} & Upper $1 / 2$ & 41.60 & 43.80 & 42.70 & & & \\
\hline$\$ 95 \mathrm{~T} 003072$ & & Lower $1 / 2$ & 39.50 & 40.30 & 39.90 & & & \\
\hline S95T003073 & \multirow[t]{2}{*}{$112: 9$} & Upper $1 / 2$ & 45.60 & 51.50 & 48.55 & & & \\
\hline S95T003074 & & Lower $1 / 2$ & 41.60 & 47.20 & 44.40 & & & \\
\hline \$95T003075 & \multirow[t]{2}{*}{$112: 10$} & Upper $1 / 2$ & 40.50 & 42.90 & 41.70 & & & \\
\hline S95T003076 & & Lower $1 / 2$ & $<45.6$ & 48.10 & 46.9 & & & \\
\hline S95T003077 & \multirow[t]{2}{*}{$112: 11$} & Upper $1 / 2$ & 44.50 & 52.70 & 48.60 & & & \\
\hline S95T003078 & & Lower $1 / 2$ & $<94.9$ & $<115$ & $<105$ & & & \\
\hline S95T003079 & \multirow[t]{2}{*}{$112: 12$} & Upper $1 / 2$ & $<107$ & $<96.6$ & $<102$ & & & \\
\hline S95T003080 & & Lower $1 / 2$ & $<97.8$ & $<101$ & $<99.4$ & & & \\
\hline
\end{tabular}


Table B-17. Tank 241-B-204 Analytical Results: Magnesium. (3 sheets)

\begin{tabular}{|c|c|c|c|c|c|c|c|c|}
\hline Kanolo & \% & $\sin 6 \sin 1$ & $\operatorname{lin}, 111$ & Bnoly.10 & intin & $4 \%$ & $\begin{array}{l}1+58 \\
(3+6)\end{array}$ & 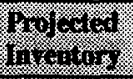 \\
\hline Solm & . & (3) & 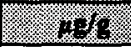 & 68 & 34 & 1/2 & 8 & (4) \\
\hline S95T003081 & \multirow[t]{2}{*}{$112: 13$} & Upper $1 / 2$ & $<87.2$ & $<87.3$ & $<87.2$ & & & \\
\hline S95T003082 & & Lower $1 / 2$ & $<97.2$ & $<84.7$ & $<91.0$ & & & \\
\hline S95T003083 & \multirow[t]{2}{*}{$112: 14$} & Upper $1 / 2$ & $<92.6$ & $<106$ & $<99.3$ & & & \\
\hline S95T003084 & & Lower $1 / 2$ & $<199$ & $<181$ & $<195$ & & & \\
\hline S95T003282 & \multirow[t]{2}{*}{$114: 2$} & Upper $1 / 2$ & 63.40 & 59.60 & 61.50 & & & \\
\hline \$95T003283 & & Lower $1 / 2$ & 30.40 & 33.70 & 32.05 & & & \\
\hline S95T003284 & \multirow[t]{2}{*}{$114: 3$} & Upper $1 / 2$ & 47.30 & 47.90 & 47.60 & & & \\
\hline S95T003285 & & Lower $1 / 2$ & $<169$ & $<193$ & $<181$ & & & \\
\hline S95T003286 & \multirow[t]{2}{*}{$114: 4$} & Upper $1 / 2$ & 54.80 & 48.10 & 51.45 & & & \\
\hline S95T003287 & & Lower $1 / 2$ & 82.90 & 45.70 & 64.30 & & & \\
\hline S95T003288 & \multirow[t]{2}{*}{$114: 5$} & Upper $1 / 2$ & 34.70 & 31.30 & 33.00 & & & \\
\hline S95T003289 & & Lower $1 / 2$ & 27.20 & 29.70 & 28.45 & & & \\
\hline S95T003290 & \multirow[t]{2}{*}{$114: 6$} & Upper $1 / 2$ & 43.40 & 35.30 & 39.35 & & & \\
\hline S95T003291 & & Lower $1 / 2$ & $<34.4$ & $<42.5$ & $<38.5$ & & & \\
\hline S95T003292 & \multirow[t]{2}{*}{$114: 7$} & Upper $1 / 2$ & 29.20 & 32.30 & 30.75 & & & \\
\hline S95T003293 & & Lower $1 / 2$ & 34.30 & 32.00 & 33.15 & & & \\
\hline S95T003294 & \multirow[t]{2}{*}{$114: 8$} & Upper $1 / 2$ & 1,240 & 312.0 & 776.0 & & & \\
\hline S95T003295 & & Lower $1 / 2$ & 31.30 & 30.70 & 31.00 & & & \\
\hline S95T003296 & \multirow[t]{2}{*}{$114: 9$} & Upper $1 / 2$ & $<41.1$ & $<46.4$ & $<43.8$ & & & \\
\hline S95T003297 & & Lower $1 / 2$ & $<37.9$ & $<45.0$ & $<41.5$ & & & \\
\hline
\end{tabular}


Table B-17. Tank 241-B-204 Analytical Results: Magnesium. (3 sheets)

\begin{tabular}{|c|c|c|c|c|c|c|c|c|}
\hline Sginos & $\log _{0}$ & $\log _{2}$ & minilin & $3_{4}$ & 812 & $\frac{6}{619}$ & 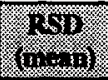 & ogrowing \\
\hline \multicolumn{2}{|l|}{ Wollos } & (2. & r. & . 63 & 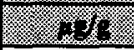 & 1. & (1) & 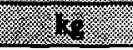 \\
\hline S95T003298 & \multirow[t]{2}{*}{$114: 10$} & Upper $1 / 2$ & $<37.0$ & $<35.9$ & $<36.5$ & & & \\
\hline S95T003299 & & Lower $1 / 2$ & 36.20 & $<38.5$ & 37.4 & & & \\
\hline S95T003300 & \multirow[t]{2}{*}{$114: 11$} & Upper $1 / 2$ & $<47.0$ & $<48.3$ & $<47.7$ & & & \\
\hline S95T003301 & & Lower $1 / 2$ & $<34.0$ & $<34.0$ & $<34.0$ & & & \\
\hline S95T003302 & \multirow[t]{2}{*}{$114: 12$} & Upper $1 / 2$ & 28.60 & 30.90 & 29.75 & & & \\
\hline S95T003303 & & Lower $1 / 2$ & 38.30 & $<37.0$ & 37.7 & & & \\
\hline S95T003304 & \multirow[t]{2}{*}{$114: 13$} & Upper $1 / 2$ & $<45.8$ & 41.90 & 43.9 & & & \\
\hline \$95T003305 & & Lower $1 / 2$ & 44.0 & $<36.6$ & 40.3 & & & \\
\hline S95T003306 & 114:14 & Upper $1 / 2$ & $<42.9$ & $<42.7$ & 42.8 & & & \\
\hline S95T003307 & & Lower $1 / 2$ & $<38.6$ & 54.20 & 46.4 & & & \\
\hline \multicolumn{2}{|c|}{ 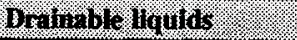 } & (2) & o/min & 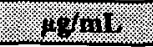 & 64117 & $18 \sin 1$ & ( & (2) \\
\hline S95T002976 & $112: 1$ & DL & $<20.1$ & $<20.1$ & $<20.1$ & \multirow[t]{3}{*}{$<15.1$} & \multirow[t]{3}{*}{$\mathrm{n} / \mathrm{a}$} & \multirow[t]{3}{*}{$<0.0604$} \\
\hline S95T002984 & $112: 2$ & $\mathrm{DL}$ & $<20.1$ & $<20.1$ & $<20.1$ & & & \\
\hline S95T003255 & $114: 1$ & $\mathrm{DL}$ & $<10.1$ & $<10.1$ & $<10.1$ & & & \\
\hline
\end{tabular}




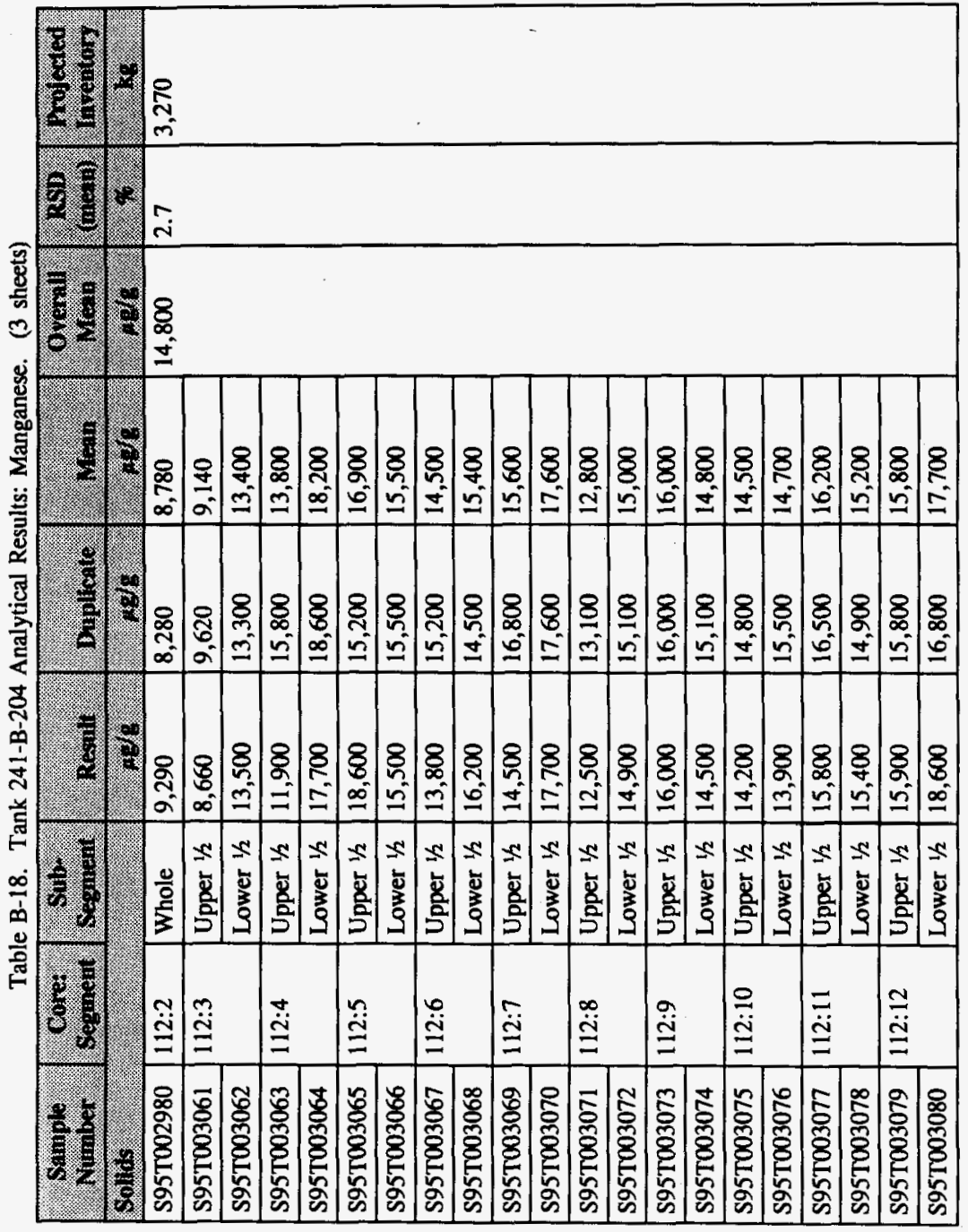




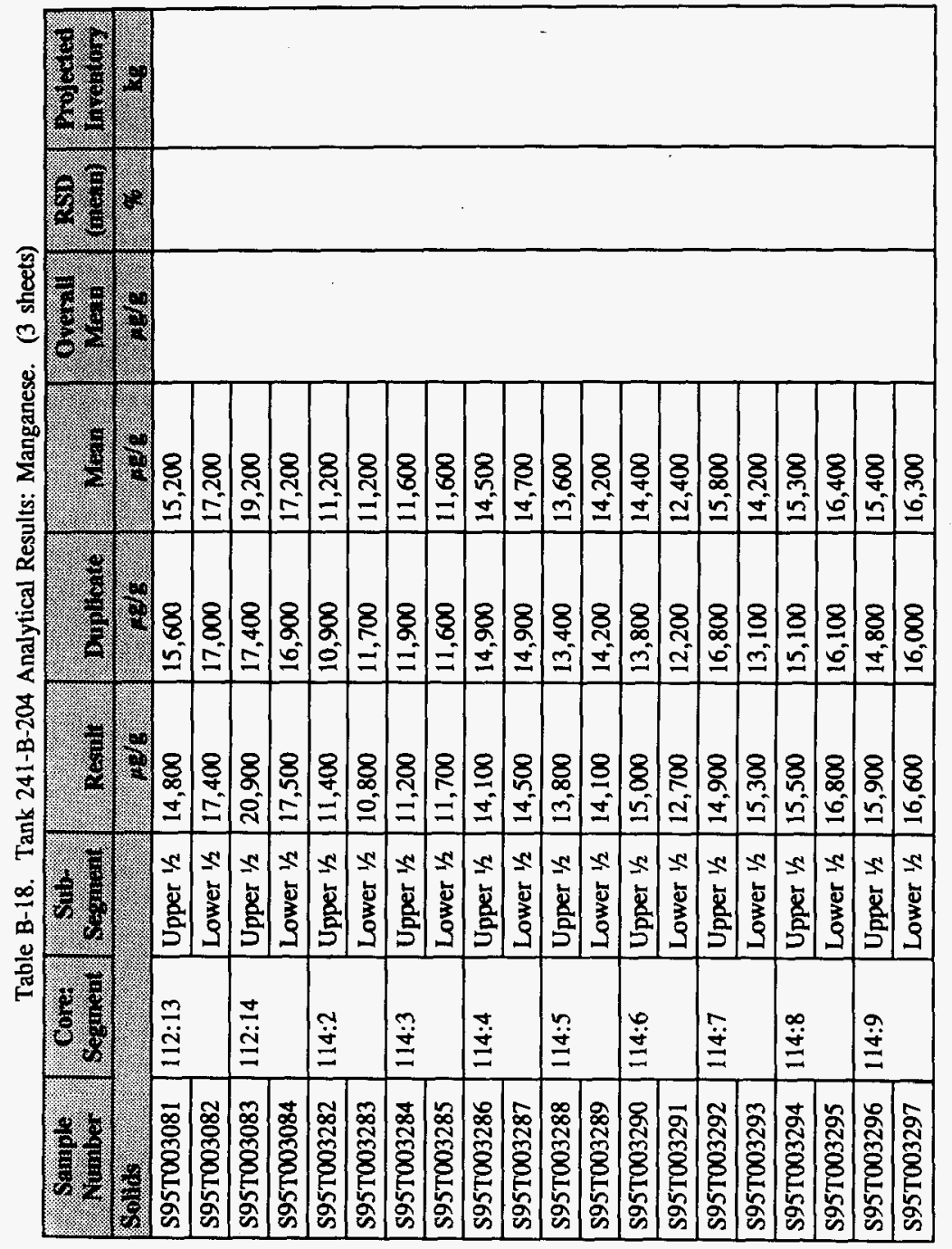


Table B-18. Tank 241-B-204 Analytical Results: Manganese. (3 sheets)

\begin{tabular}{|c|c|c|c|c|c|c|c|c|}
\hline Manjor & 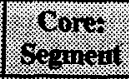 & 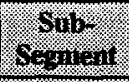 & menili & Dinilene & $3=1$ & $4 x+1$ & 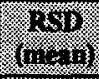 & 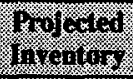 \\
\hline Q01: & & 94 & 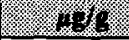 & 45 & 34 & $1 \%$ & 4 & 16 \\
\hline S95T003298 & \multirow[t]{2}{*}{$114: 10$} & Upper $1 / 2$ & 16,000 & 14,900 & 15,400 & & & \\
\hline S95T003299 & & Lower $1 / 2$ & 14,900 & 15,000 & 15,000 & & & \\
\hline S95T003300 & \multirow[t]{2}{*}{$114: 11$} & Upper $1 / 2$ & 16,600 & 15,800 & 16,200 & & & \\
\hline S95T003301 & & Lower $1 / 2$ & 16,500 & 16,100 & 16,300 & & & \\
\hline S95T003302 & \multirow[t]{2}{*}{$114: 12$} & Upper $1 / 2$ & 13,900 & 15,500 & 14,700 & & & \\
\hline \$95T003303 & & Lower $1 / 2$ & 17,400 & 16,200 & 16,800 & & & \\
\hline \$95T003304 & \multirow[t]{2}{*}{$114: 13$} & Upper $1 / 2$ & 17,700 & 18,600 & 18,200 & & & \\
\hline S95T003305 & & Lower $1 / 2$ & 15,800 & 16,000 & 15,900 & & & \\
\hline \$95T003306 & \multirow[t]{2}{*}{$114: 14$} & Upper $1 / 2$ & 15,700 & 15,400 & 15,600 & & & \\
\hline \$95T003307 & & Lower $1 / 2$ & 15,400 & 16,400 & 15,900 & & & \\
\hline \multicolumn{3}{|c|}{ Bryingle ligulas } & (2) & (2)/1i1 & (1) & Hint & (3. & 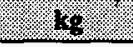 \\
\hline \$95T002976 & $112: 1$ & $\mathrm{DL}$ & $<2.01$ & $<2.01$ & $<2.01$ & \multirow[t]{3}{*}{$<1.51$} & \multirow[t]{3}{*}{$n / a$} & \multirow[t]{3}{*}{$<0.00604$} \\
\hline \$95T002984 & $112: 2$ & DL & $<2.01$ & $<2.01$ & $<2.01$ & & & \\
\hline S95T003255 & $114: 1$ & $\mathrm{DL}$ & $<1.01$ & $<1.01$ & $<1.01$ & & & \\
\hline
\end{tabular}


WHC-SD-WM-ER-581 Rev. 0

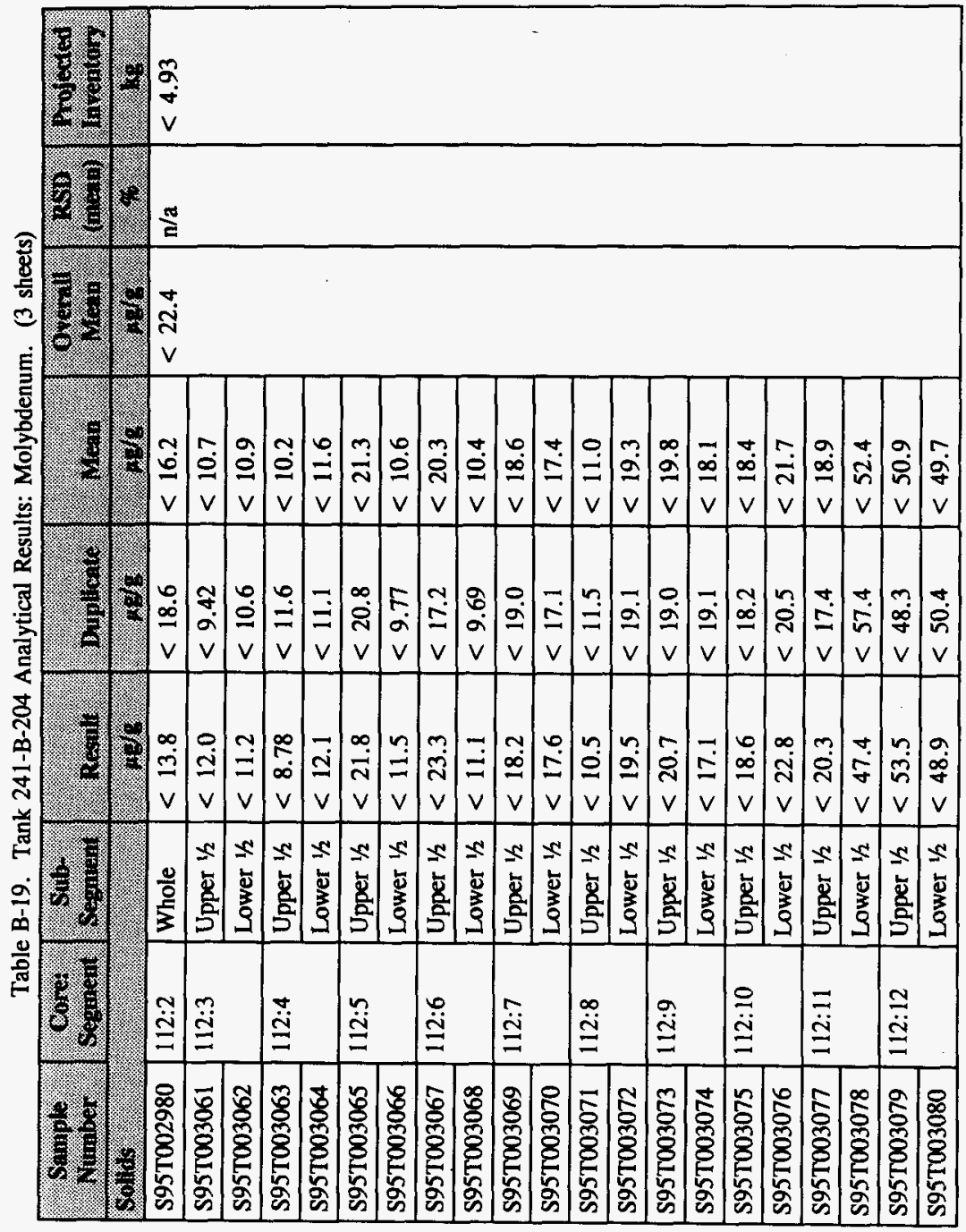


Table B-19. Tank 241-B-204 Analytical Results: Molybdenum. (3 sheets)

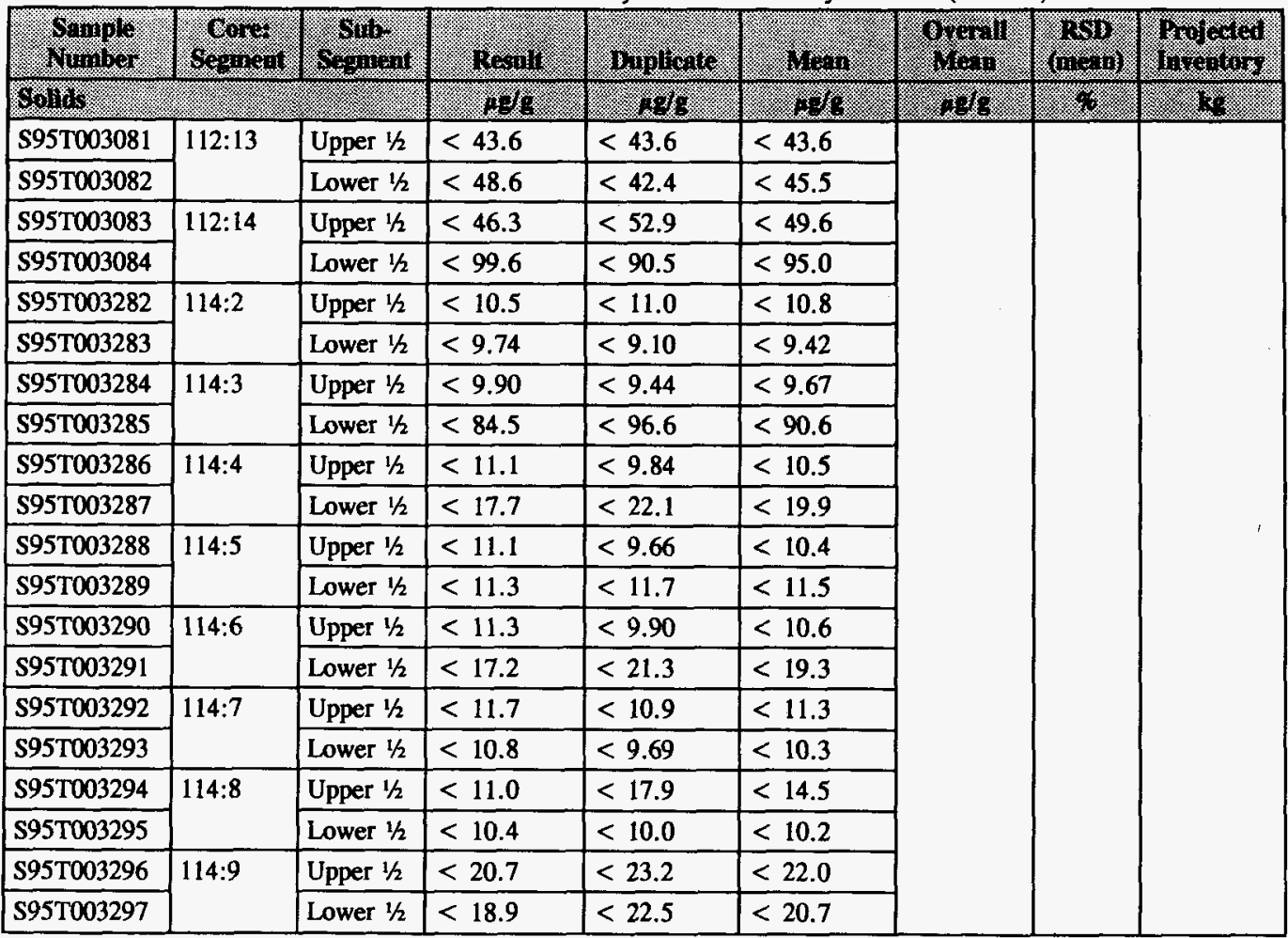


WHC-SD-WM-ER-581 Rev. 0

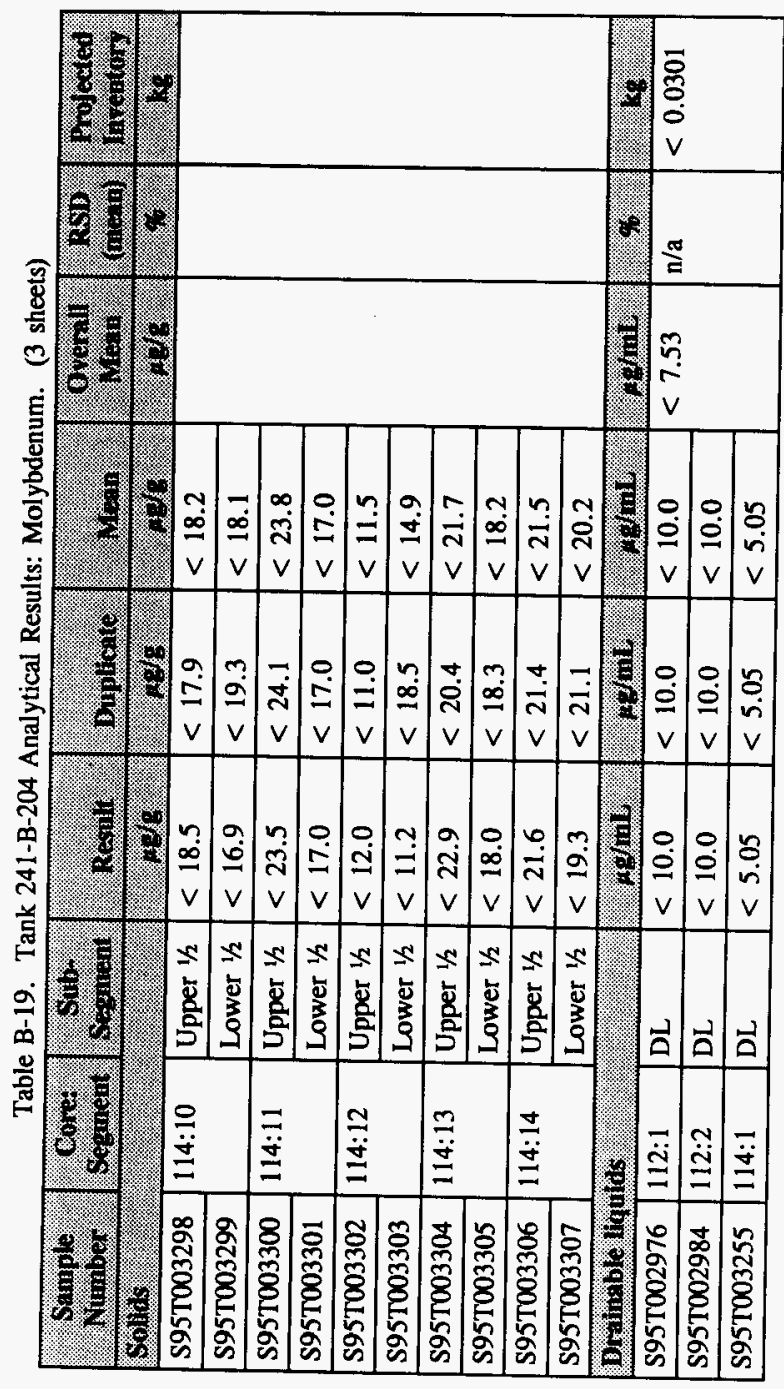




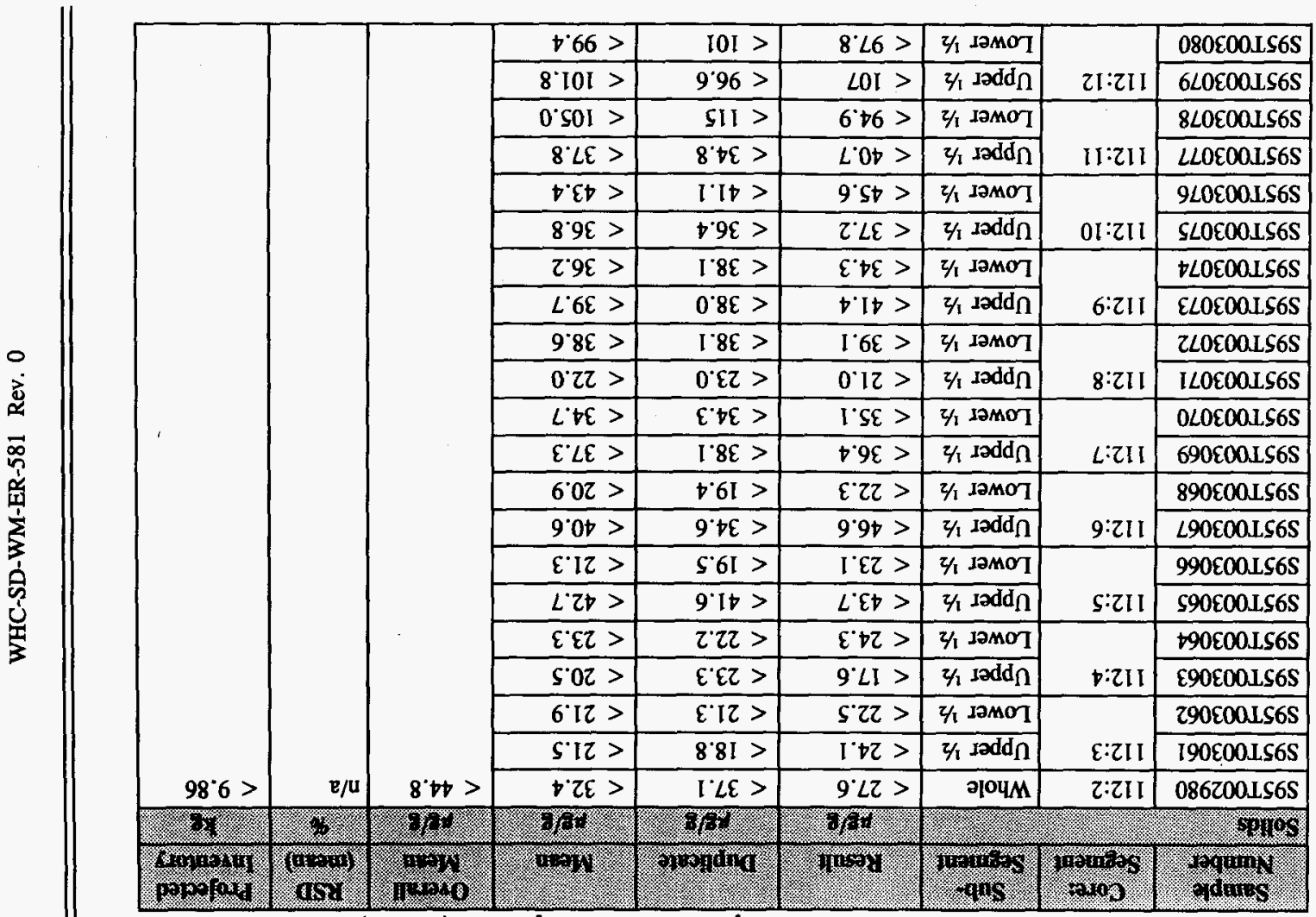

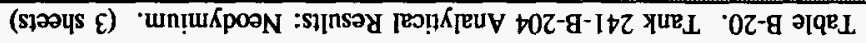




\begin{tabular}{|c|c|c|c|c|c|c|c|c|}
\hline & & & S.It > & $0 . S t>$ & $6 \angle \varepsilon>$ & z/1 IәMOI & \multirow[b]{2}{*}{$6: t I I$} & $\angle 62 \varepsilon 00 \mathrm{LS} 6 \mathrm{~S}$ \\
\hline & & & $8^{\circ} \varepsilon t>$ & $79 t>$ & I. It $>$ & $z / 1$ sadd $\Omega$ & & 96ZE00LS6S \\
\hline & & & $\varepsilon 0 z>$ & $0.02>$ & $5.02>$ & $4 / 1$ IOMOT & \multirow[b]{2}{*}{$8: t I I$} & S6ZE00LS6S \\
\hline & & & $8.82>$ & $8 . S E>$ & $L I I Z>$ & $2 / 1$ sadd $\Pi$ & & †6ZE00LS6S \\
\hline & & & $502>$ & $761>$ & $9 \cdot 12>$ & $2 / 1$ dәMOT & \multirow[b]{2}{*}{$L: D I I$} & E6ZE00LS6S \\
\hline & & & $9.22>$ & $L \cdot 12>$ & $\nabla \varepsilon Z>$ & $2 / 1$ Iadd & & Z62800LS6S \\
\hline & & & $S^{\circ} 8 E>$ & $s z b>$ & $\nabla^{\circ} \forall \varepsilon>$ & $z / 1$ JəMO & \multirow[b]{2}{*}{$9: t I I$} & I6ZE00.LS6S \\
\hline & & & $2.12>$ & $8.61>$ & $922>$ & $\psi_{1}$ Iadd $\Omega$ & & 06ZE00LS6S \\
\hline & & & $0 . \varepsilon Z>$ & $t^{\circ} \varepsilon z>$ & $9 . z z>$ & $2 / 1$ JәMOT & \multirow[b]{2}{*}{$s: t I I$} & 682E00LS6S \\
\hline & & & $802>$ & $\varepsilon 6 I>$ & $2.22>$ & $2 / 1$ Iadd $\Omega$ & & 88ZE00LS6S \\
\hline & & & $L^{\circ} 6 \varepsilon>$ & $\varepsilon \neq t>$ & I'SE > & $2 / 12 \mathrm{MO}$ & \multirow[b]{2}{*}{$t: t t I$} & $\angle 8 Z E 00 L S 6 S$ \\
\hline & & & $0.12>$ & $L 61>$ & $\varepsilon z 2>$ & $z_{1} \operatorname{Iadd}_{\Omega}$ & & 98ZE00LS6S \\
\hline & & & $0.181>$ & E6I $>$ & $69 I>$ & $2 / 1$ Iәм0 & \multirow[b]{2}{*}{$\varepsilon: t I I$} & S8ZE00LS6S \\
\hline & & & $76 \mathrm{I}>$ & $681>$ & $8.61>$ & $z / 1$ Iadd $\Omega$ & & t8ZE00LS6S \\
\hline & & & $681>$ & $281>$ & $561>$ & Z/ IวMO & \multirow[b]{2}{*}{$Z: t I I$} & E8ZE00LS6S \\
\hline & & & $s^{\prime} i z>$ & $0.22>$ & $0 . I Z>$ & z/1 sadd $\Omega$ & & Z8ZE00LS6S \\
\hline & & & $0.061>$ & I8I $>$ & $66 I>$ & $4 / 1$ IәMO & \multirow[b]{2}{*}{ tI:ZI I } & t80E00LS6S \\
\hline & & & $\varepsilon^{\prime} 66>$ & $901>$ & $9.26>$ & $z_{1}$ Iədd $\Omega$ & & E80E00LS6S \\
\hline & & & $0.16>$ & $L+8>$ & $2.26>$ & $\mathrm{z} / \mathrm{I}$ IอMO $\mathrm{T}$ & \multirow[b]{2}{*}{$\varepsilon I: Z I I$} & 280E00LS6S \\
\hline & & & $\varepsilon^{\prime} \angle 8>$ & $\varepsilon \angle 8>$ & $2 \angle 8>$ & $z_{1} \operatorname{sadd}_{\Omega}$ & & I80E00.LS6S \\
\hline 4 & $\sqrt{4}$. & $9 \%$ & W\% & W & 1\%? & (1) & \multirow{2}{*}{ Imomeros? } & (1) 9109 \\
\hline mingom & $\frac{9}{6434}$ & 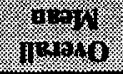 & 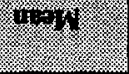 & 9791619 & 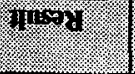 & 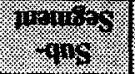 & & oforings \\
\hline
\end{tabular}


Table B-20. Tank 241-B-204 Analytical Results: Neodymium. (3 sheets)

\begin{tabular}{|c|c|c|c|c|c|c|c|c|}
\hline (5ing) & orang & 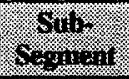 & Itecinil & 8.1310.6. & $\%=1$ & 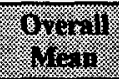 & 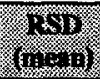 & 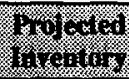 \\
\hline Sollis & & (2) & 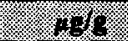 & 43 & (1): & 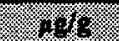 & (1) & 6 \\
\hline S95T003298 & \multirow[t]{2}{*}{$114: 10$} & Upper $1 / 2$ & $<37.0$ & $<35.9$ & $<36.5$ & & & \\
\hline$\$ 95 T 003299$ & & Lower $1 / 2$ & $<33.9$ & $<38.5$ & $<36.2$ & & & \\
\hline S95T003300 & \multirow[t]{2}{*}{$114: 11$} & Upper $1 / 2$ & $<47.0$ & $<48.3$ & $<47.7$ & & & \\
\hline S95T003301 & & Lower $1 / 2$ & $<34.0$ & $<34.0$ & $<34.0$ & & & \\
\hline \$95T003302 & \multirow[t]{2}{*}{$114: 12$} & Upper $1 / 2$ & $<24.0$ & $<22.1$ & $<23.1$ & & & \\
\hline$\$ 95 T 003303$ & & Lower $1 / 2$ & $<22.3$ & $<37.0$ & $<29.7$ & & & \\
\hline \$95T003304 & \multirow[t]{2}{*}{$114: 13$} & Upper $1 / 2$ & $<45.8$ & $<40.9$ & $<43.4$ & & & \\
\hline \$95T003305 & & Lower $1 / 2$ & $<36.0$ & $<36.6$ & $<36.3$ & & & \\
\hline S95T003306 & \multirow[t]{2}{*}{$114: 14$} & Upper $1 / 2$ & $<42.9$ & $<42.7$ & $<42.8$ & & & \\
\hline S95T003307 & & Lower $1 / 2$ & $<38.6$ & $<42.2$ & $<40.4$ & & & \\
\hline \multicolumn{2}{|c|}{ Dratinble ligulds } & 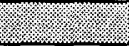 & Pdin & (1) & $(52)$ & 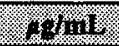 & 46 & (3) \\
\hline S95T002976 & $112: 1$ & $\mathrm{DL}$ & $<20.1$ & $<20.1$ & $<20.1$ & \multirow[t]{3}{*}{$<15.1$} & \multirow[t]{3}{*}{$n / a$} & \multirow[t]{3}{*}{$<0.0604$} \\
\hline \$95T002984 & $112: 2$ & $\mathrm{DL}$ & $<20.1$ & $<20.1$ & $<20.1$ & & & \\
\hline S95T003255 & $114: 1$ & $\mathrm{DL}$ & $<10.1$ & $<10.1$ & $<10.1$ & & & \\
\hline
\end{tabular}


Table B-21. Tank 241-B-204 Analytical Results: Nickel. (3 sheets)

\begin{tabular}{|c|c|c|c|c|c|c|c|c|}
\hline Oinor & ofor & 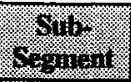 & 4. & B. & 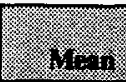 & $x_{1}$ & 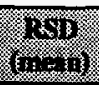 & 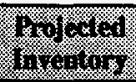 \\
\hline $\operatorname{ton} 3$ & (1) & & 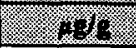 & . 15 & (3) & $(1 / 4)$ & r. & 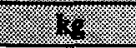 \\
\hline S95T002980 & $112: 2$ & Whole & 189.0 & 177.0 & 183.0 & \multirow[t]{21}{*}{234} & \multirow[t]{21}{*}{10.6} & \multirow[t]{21}{*}{51.5} \\
\hline S95T003061 & \multirow[t]{2}{*}{$112: 3$} & Upper $1 / 2$ & 102.0 & 104.0 & 103.0 & & & \\
\hline S95T003062 & & Lower $1 / 2$ & 169.0 & 165.0 & 167.0 & & & \\
\hline S95T003063 & \multirow[t]{2}{*}{$112: 4$} & Upper $1 / 2$ & 112.0 & 150.0 & 131.0 & & & \\
\hline S95T003064 & & Lower $1 / 2$ & 140.0 & 142.0 & 141.0 & & & \\
\hline S95T003065 & \multirow[t]{2}{*}{$112: 5$} & Upper $1 / 2$ & 208.0 & 172 & 190 & & & \\
\hline S95T003066 & & Lower $1 / 2$ & 149.0 & 145.0 & 147.0 & & & \\
\hline \$95T003067 & \multirow[t]{2}{*}{$112: 6$} & Upper $1 / 2$ & 174.0 & 184.0 & 179.0 & & & \\
\hline S95T003068 & & Lower $1 / 2$ & 181.0 & 161.0 & 171.0 & & & \\
\hline S95T003069 & \multirow[t]{2}{*}{$112: 7}$. & Upper $1 / 2$ & 166.0 & 197.0 & 181.5 & & & \\
\hline S95T003070 & & Lower $1 / 2$ & 216.0 & 210.0 & 213.0 & & & \\
\hline S95T003071 & \multirow[t]{2}{*}{$112: 8$} & Upper $1 / 2$ & 135.0 & 144.0 & 139.5 & & & \\
\hline S95T003072 & & Lower $1 / 2$ & 178.0 & 182.0 & 180.0 & & & \\
\hline S95T003073 & \multirow[t]{2}{*}{$112: 9$} & Upper $1 / 2$ & 201.0 & 207.0 & 204.0 & & & \\
\hline \$95T003074 & & Lower $1 / 2$ & 187.0 & 200.0 & 193.5 & & & \\
\hline S95T003075 & \multirow[t]{2}{*}{$112: 10$} & Upper $1 / 2$ & 229.0 & 237.0 & 233.0 & & & \\
\hline S95T003076 & & Lower $1 / 2$ & 462.0 & 476.0 & 469.0 & & & \\
\hline \$95T003077 & \multirow[t]{2}{*}{$112: 11$} & Upper $1 / 2$ & 735.0 & 786.0 & 760.5 & & & \\
\hline S95T003078 & & Lower $1 / 2$ & 509.0 & 516.0 & 512.5 & & & \\
\hline S95T003079 & \multirow[t]{2}{*}{$112: 12$} & Upper $1 / 2$ & 297.0 & 303.0 & 300.0 & & & \\
\hline S95T003080 & & Lower $1 / 2$ & 334.0 & 334.0 & 334.0 & & & \\
\hline
\end{tabular}




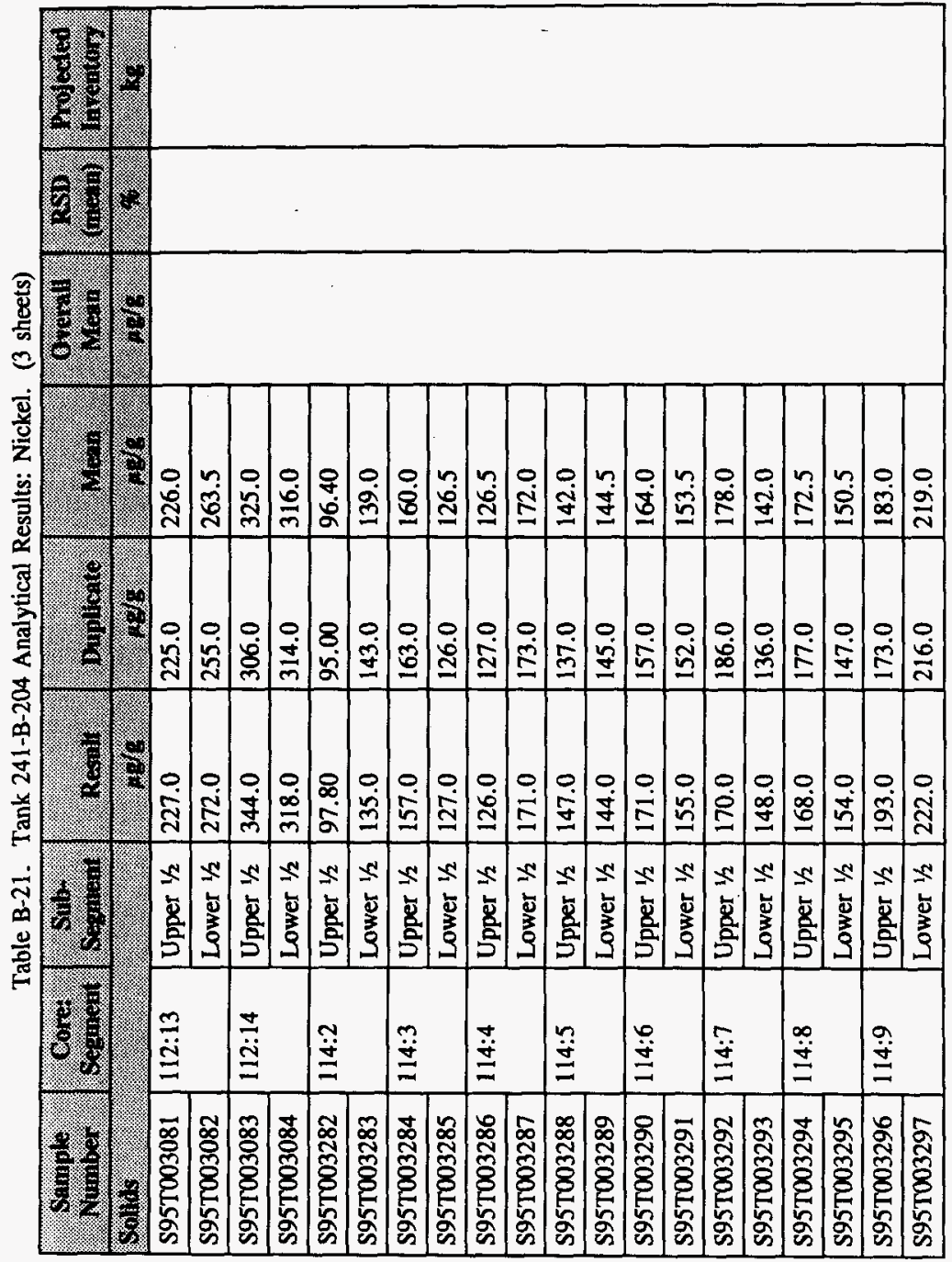


Table B-21. Tank 241-B-204 Analytical Results: Nickel. (3 sheets)

\begin{tabular}{|c|c|c|c|c|c|c|c|c|}
\hline 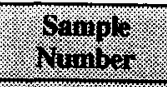 & $\frac{6}{60 x}$ & (x) & Mismin & 10p1: & (3) & 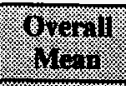 & $\begin{array}{l}243 \\
11011\end{array}$ & How corom \\
\hline 5010.6 & ? & 8 & (-) & $.21 \mathrm{C}$ & 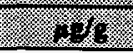 & 40 & . & (1) \\
\hline S95T003298 & \multirow[t]{2}{*}{$114: 10$} & Upper $1 / 2$ & 228.0 & 209.0 & 218.5 & & & \\
\hline S95T003299 & & Lower $1 / 2$ & 376.0 & 378.0 & 377.0 & & & \\
\hline S95T003300 & \multirow[t]{2}{*}{$114: 11$} & Upper $1 / 2$ & 776.0 & 754.0 & 765.0 & & & \\
\hline S95T003301 & & Lower $1 / 2$ & 374.0 & 364.0 & 369.0 & & & \\
\hline S95T003302 & \multirow[t]{2}{*}{$114: 12$} & Upper $1 / 2$ & 262.0 & 277.0 & 269.5 & & & \\
\hline S95T003303 & & Lower $1 / 2$ & 269.0 & 273.0 & 271.0 & & & \\
\hline S95T003304 & \multirow[t]{2}{*}{$114: 13$} & Upper $1 / 2$ & 252.0 & 257.0 & 254.5 & & & \\
\hline S95T003305 & & Lower $1 / 2$ & 211.0 & 216.0 & 213.5 & & & \\
\hline S95T003306 & \multirow[t]{2}{*}{$114: 14$} & Upper $1 / 2$ & 251.0 & 250.0 & 250.5 & & & \\
\hline S95T003307 & & Lower $1 / 2$ & 261.0 & 274.0 & 267.5 & & & \\
\hline \multicolumn{3}{|c|}{ Brinalo lajus } & $181 \mathrm{mil}$ & \% 1 min & (8) & onint & 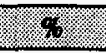 & 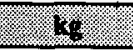 \\
\hline \$95T002976 & 112:1 & $\overline{D L}$ & $<4.02$ & $<4.02$ & $<4.02$ & \multirow[t]{3}{*}{$<3.02$} & \multirow[t]{3}{*}{$n / a$} & \multirow[t]{3}{*}{$<0.0121$} \\
\hline S95T002984 & $112: 2$ & $\mathrm{DL}$ & $<4.02$ & $<4.02$ & $<4.02$ & & & \\
\hline S95T003255 & $114: 1$ & DL & $<2.02$ & $<2.02$ & $<2.02$ & & & \\
\hline
\end{tabular}


WHC-SD-WM-ER-581 Rev. 0

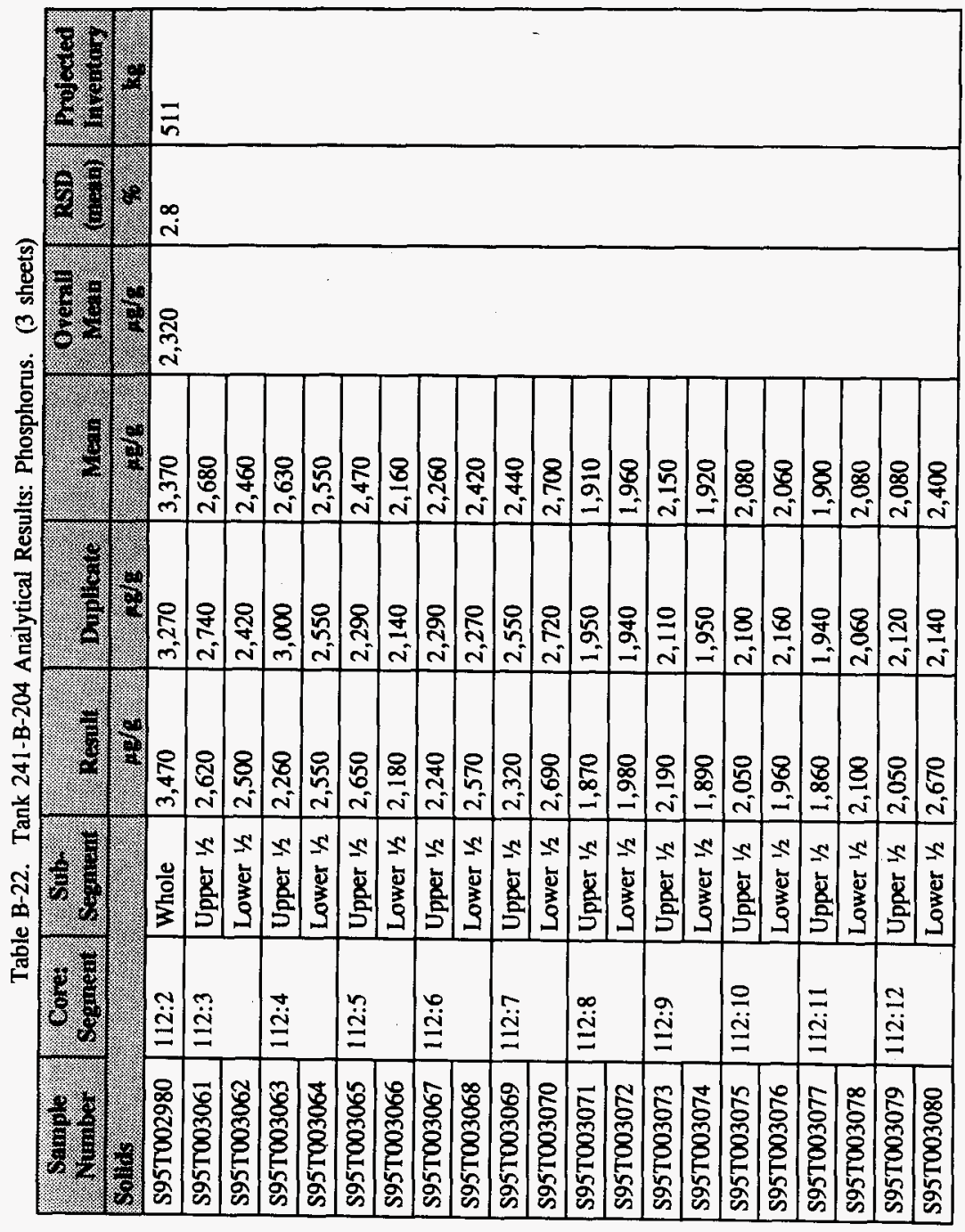


Table B-22. Tank 241-B-204 Analytical Results: Phosphorus. (3 sheets)

\begin{tabular}{|c|c|c|c|c|c|c|c|c|}
\hline STinplor & 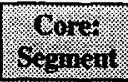 & 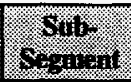 & icsall & Binglare & $18+2$ & 6\%, & $\frac{1+31}{(\operatorname{sic} 1)}$ & 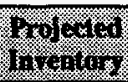 \\
\hline Won & & (1) & 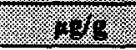 & . & (vis & $-1 f_{5}$ & . & (1) \\
\hline S95T003081 & \multirow[t]{2}{*}{$112: 13$} & Upper $1 / 2$ & 1,990 & 2,110 & 2,050 & & & \\
\hline S95T003082 & & Lower $1 / 2$ & 2,430 & 2,300 & 2,360 & & & \\
\hline S95T003083 & \multirow[t]{2}{*}{$112: 14$} & Upper $1 / 2$ & 2,750 & 2,030 & 2,390 & & & \\
\hline S95T003084 & & Lower $1 / 2$ & 2,270 & 2,060 & 2,160 & & & \\
\hline \$95T003282 & \multirow[t]{2}{*}{$114: 2$} & Upper $1 / 2$ & 3,380 & 3,230 & 3,300 & & & \\
\hline S95T003283 & & Lower $1 / 2$ & 2,690 & 2,820 & 2,760 & & & \\
\hline S95T003284 & \multirow[t]{2}{*}{$114: 3$} & Upper $1 / 2$ & 2,230 & 2,220 & 2,220 & & & \\
\hline S95T003285 & & Lower $1 / 2$ & 2,670 & 2,680 & 2,680 & & & \\
\hline S95T003286 & \multirow[t]{2}{*}{$114: 4$} & Upper $1 / 2$ & 2,420 & 2,450 & 2,440 & & & \\
\hline S95T003287 & & Lower $1 / 2$ & 2,390 & 2,470 & 2,430 & & & \\
\hline S95T003288 & \multirow[t]{2}{*}{$114: 5$} & Upper $1 / 2$ & 2,390 & 2,260 & 2,320 & & & \\
\hline \$95T003289 & & Lower $1 / 2$ & 2,570 & 2,520 & 2,540 & & & \\
\hline S95T003290 & \multirow[t]{2}{*}{$114: 6$} & Upper $1 / 2$ & 2,670 & 2,380 & 2,520 & & & \\
\hline S95T003291 & & Lower $1 / 2$ & 2,300 & 2,300 & 2,300 & & & \\
\hline S95T003292 & \multirow[t]{2}{*}{$114: 7$} & Upper $1 / 2$ & 2,560 & 2,720 & 2,640 & & & \\
\hline S95T003293 & & Lower $1 / 2$ & 2,430 & 2,170 & 2,300 & & & \\
\hline \$95T003294 & \multirow[t]{2}{*}{$114: 8$} & Upper $1 / 2$ & 2,520 & 2,610 & 2,560 & & & \\
\hline S95T003295 & & Lower $1 / 2$ & 2,380 & 2,260 & 2,320 & & & \\
\hline S95T003296 & \multirow[t]{2}{*}{$114: 9$} & Upper $1 / 2$ & 2,310 & 2,250 & 2,280 & & & \\
\hline S95T003297 & & Lower $1 / 2$ & 2,370 & 2,370 & 2,370 & & & \\
\hline
\end{tabular}


Table B-22. Tank 241-B-204 Analytical Results: Phosphorus. (3 sheets)

\begin{tabular}{|c|c|c|c|c|c|c|c|c|}
\hline 6impl- & 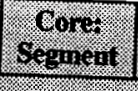 & 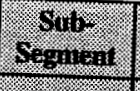 & $16.1 \%$ & 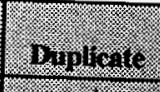 & 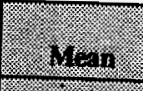 & 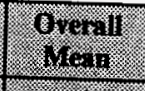 & $\frac{1}{1011}=$ & 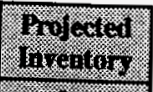 \\
\hline 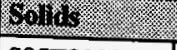 & & & (3) & $y_{-1}$ & (2) & 2015 & . & . \\
\hline S95T003298 & \multirow[t]{2}{*}{$114: 10$} & Upper $1 / 2$ & 2,210 & 2,180 & 2,200 & & & \\
\hline S95T003299 & & Lower $1 / 2$ & 1,990 & 2,010 & 2,000 & & & \\
\hline S95T003300 & \multirow[t]{2}{*}{$114: 11$} & Upper $1 / 2$ & 2,120 & 2,000 & 2,060 & & & \\
\hline S95\% & & Lower $1 / 2$ & 2,110 & 1,960 & 2,040 & & & \\
\hline S95T003302 & \multirow[t]{2}{*}{$114: 12$} & Upper $1 / 2$ & 1,770 & 1,870 & 1,820 & & & \\
\hline S95T003303 & & Lower $1 / 2$ & 1,950 & 2,000 & 1,980 & & & \\
\hline S95T003304 & \multirow[t]{2}{*}{$114: 13$} & Upper $1 / 2$ & 2,090 & 2,300 & 2,200 & & & \\
\hline S95T003305 & & Lower $1 / 2$ & 1,970 & 2,020 & 2,000 & & & \\
\hline S95T003306 & \multirow[t]{2}{*}{$114: 14$} & Upper $1 / 2$ & 1,790 & 1,780 & 1,780 & & & \\
\hline \$95T003307 & & Lower $1 / 2$ & 1,770 & 1,880 & 1,820 & & & \\
\hline \multicolumn{3}{|c|}{ Drifrobiviguth } & H/113 & (2) & 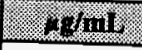 & $241 \mathrm{ni}$ & . & $\mathrm{k}$ \\
\hline S95T002976 & 112:1 & DL & 688.0 & 690.0 & 689.0 & \multirow[t]{3}{*}{680} & \multirow[t]{3}{*}{1.0} & \multirow[t]{3}{*}{2.72} \\
\hline S95T002984 & $112: 2$ & $\mathrm{DL}$ & 678.0 & 653.0 & 665.5 & & & \\
\hline S95T003255 & $114: 1$ & $\mathrm{DL}$ & 697.0 & 667.0 & 682.0 & & & \\
\hline
\end{tabular}


Table B-23. Tank 241-B-204 Analytical Results: Potassium. (3 sheets)

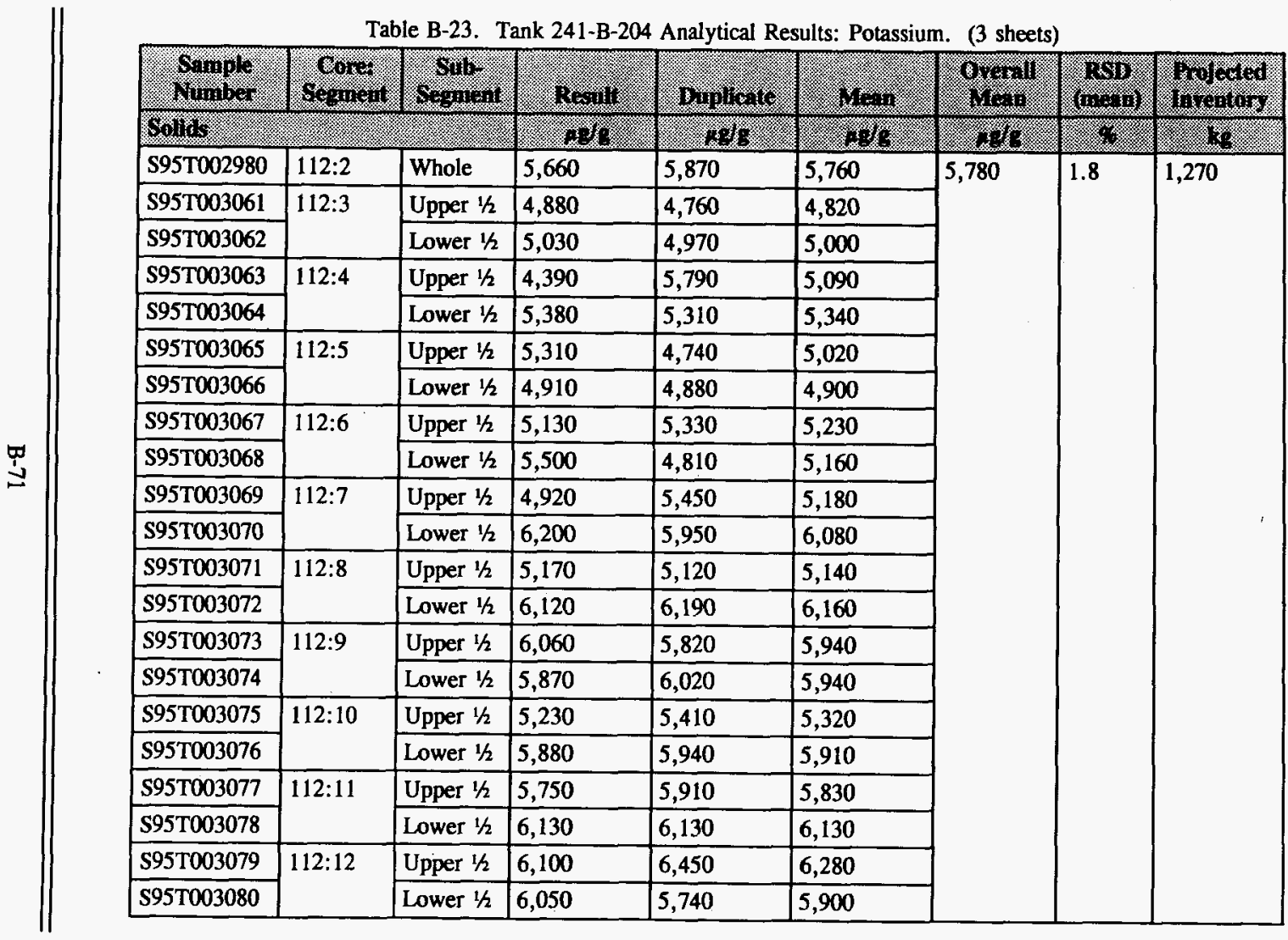


Table B-23. Tank 241-B-204 Analytical Results: Potassium. (3 sheets)

\begin{tabular}{|c|c|c|c|c|c|c|c|c|}
\hline Gompor & 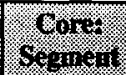 & 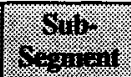 & Timin: & Bipirente & 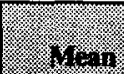 & ong & $\frac{1}{453}$ & 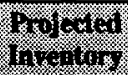 \\
\hline Wilin: & & (3) & 181 & 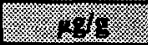 & 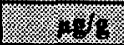 & .46 & 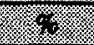 & 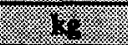 \\
\hline S95T003081 & \multirow[t]{2}{*}{$112: 13$} & Upper $1 / 2$ & 5,960 & 5,860 & 5,910 & & & \\
\hline S95T003082 & & Lower $1 / 2$ & 6,620 & 6,470 & 6,540 & & & \\
\hline \$95T003083 & \multirow[t]{2}{*}{$112: 14$} & Upper $1 / 2$ & 6,590 & 6,330 & 6,460 & & & \\
\hline S95T003084 & & Lower $1 / 2$ & 7,060 & 6,820 & 6,940 & & & \\
\hline S95T003282 & \multirow[t]{2}{*}{$114: 2$} & Upper $1 / 2$ & 5,320 & 5,140 & 5,230 & & & \\
\hline S95T003283 & & Lower $1 / 2$ & 5,040 & 5,180 & 5,110 & & & \\
\hline S95T003284 & \multirow[t]{2}{*}{$114: 3$} & Upper $1 / 2$ & 5,140 & 5,190 & 5,160 & & & \\
\hline s95T003285 & & Lower $1 / 2$ & 5,540 & 5,860 & 5,700 & & & \\
\hline S95T003286 & \multirow[t]{2}{*}{$114: 4$} & Upper $1 / 2$ & 4,920 & 4,870 & 4,900 & & & \\
\hline S95T003287 & & Lower $1 / 2$ & 5,620 & 5,620 & 5,620 & & & . \\
\hline S95T003288 & \multirow[t]{2}{*}{$114: 5$} & Upper $1 / 2$ & 5,660 & 5,420 & 5,540 & & & \\
\hline S95T003289 & & Lower $1 / 2$ & 5,830 & 5,850 & 5,840 & & & \\
\hline S95T003290 & \multirow[t]{2}{*}{$114: 6$} & Upper $1 / 2$ & 6,110 & 5,850 & 5,980 & & & \\
\hline S95T003291 & & Lower $1 / 2$ & 5,750 & 5,700 & 5,720 & & & \\
\hline \$95T003292 & \multirow[t]{2}{*}{$114: 7$} & Upper $1 / 2$ & 5,410 & 5,720 & 5,560 & & & \\
\hline S95T003293 & & Lower $1 / 2$ & 6,160 & 5,630 & 5,900 & & & \\
\hline S95T003294 & \multirow[t]{2}{*}{$114: 8$} & Upper $1 / 2$ & 5,520 & 5,980 & 5,750 & & & \\
\hline S95T003295 & & Lower $1 / 2$ & 5,520 & 5,330 & 5,420 & & & \\
\hline S95T003296 & \multirow[t]{2}{*}{$114: 9$} & Upper $1 / 2$ & 6,180 & 5,960 & 6,070 & & & \\
\hline S95T003297 & & Lower $1 / 2$ & 6,180 & 6,020 & 6,100 & & & \\
\hline
\end{tabular}


Table B-23. Tank 241-B-204 Analytical Results: Potassium. (3 sheets)

\begin{tabular}{|c|c|c|c|c|c|c|c|c|}
\hline Wump & obout & 40 & Perinil: & mojisate & Hit.n & 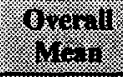 & 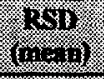 & 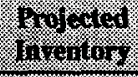 \\
\hline onos & & (1) & (2) & 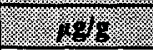 & 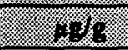 & 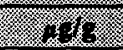 & . & 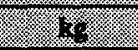 \\
\hline S95T003298 & \multirow[t]{2}{*}{$114: 10$} & Upper $1 / 2$ & 6,360 & 6,060 & 6,210 & & & \\
\hline$\$ 95 T 003299$ & & Lower $1 / 2$ & 6,370 & 6,360 & 6,360 & & & \\
\hline S95T003300 & \multirow[t]{2}{*}{$114: 11$} & Upper $1 / 2$ & 6,540 & 6,560 & 6,550 & & & \\
\hline \$95T003301 & & Lower $1 / 2$ & 6,840 & 6,540 & 6,690 & & & \\
\hline S95T003302 & \multirow[t]{2}{*}{$114: 12$} & Upper $1 / 2$ & 6,290 & 6,470 & 6,380 & & & \\
\hline $595 \mathrm{~T} 003303$ & & Lower $1 / 2$ & 6,590 & 6,700 & 6,640 & & & \\
\hline$\$ 957003304$ & \multirow[t]{2}{*}{$114: 13$} & Upper $1 / 2$ & 7,160 & 7,030 & 7,100 & & & \\
\hline S95T003305 & & Lower $1 / 2$ & 6,000 & 6,040 & 6,020 & & & \\
\hline S95T003306 & \multirow[t]{2}{*}{$114: 14$} & Upper $1 / 2$ & 5,760 & 5,960 & 5,860 & & & \\
\hline S95T003307 & & Lower $1 / 2$ & 5,470 & 5,750 & 5,610 & & & \\
\hline \multicolumn{3}{|c|}{ Braliable lquids } & $10 / 111$ & (c) & a / & HYil & 8. & 18 \\
\hline \$95T002976 & $112: 1$ & $\mathrm{DL}$ & 6,080 & 6,010 & 6,040 & \multirow[t]{3}{*}{6,110} & \multirow[t]{3}{*}{4.6} & \multirow[t]{3}{*}{24.4} \\
\hline S95T002984 & $112: 2$ & $\mathrm{DL}$ & 5,600 & 5,600 & 5,600 & & & \\
\hline S95T003255 & $114: 1$ & $\mathrm{DL}$ & 6,480 & 6,300 & 6,390 & & & \\
\hline
\end{tabular}




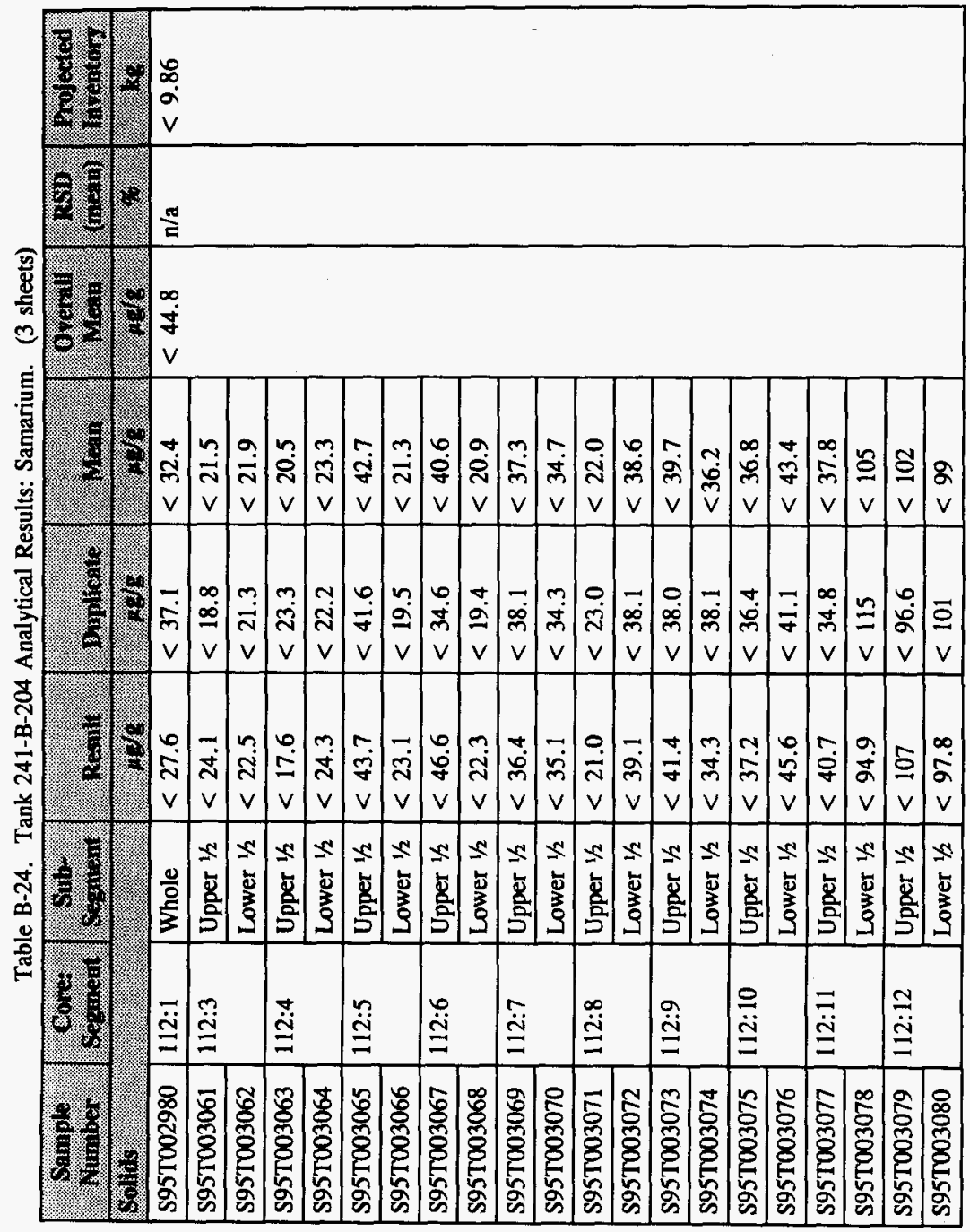


WHC-SD-WM-ER-581 Rev. 0

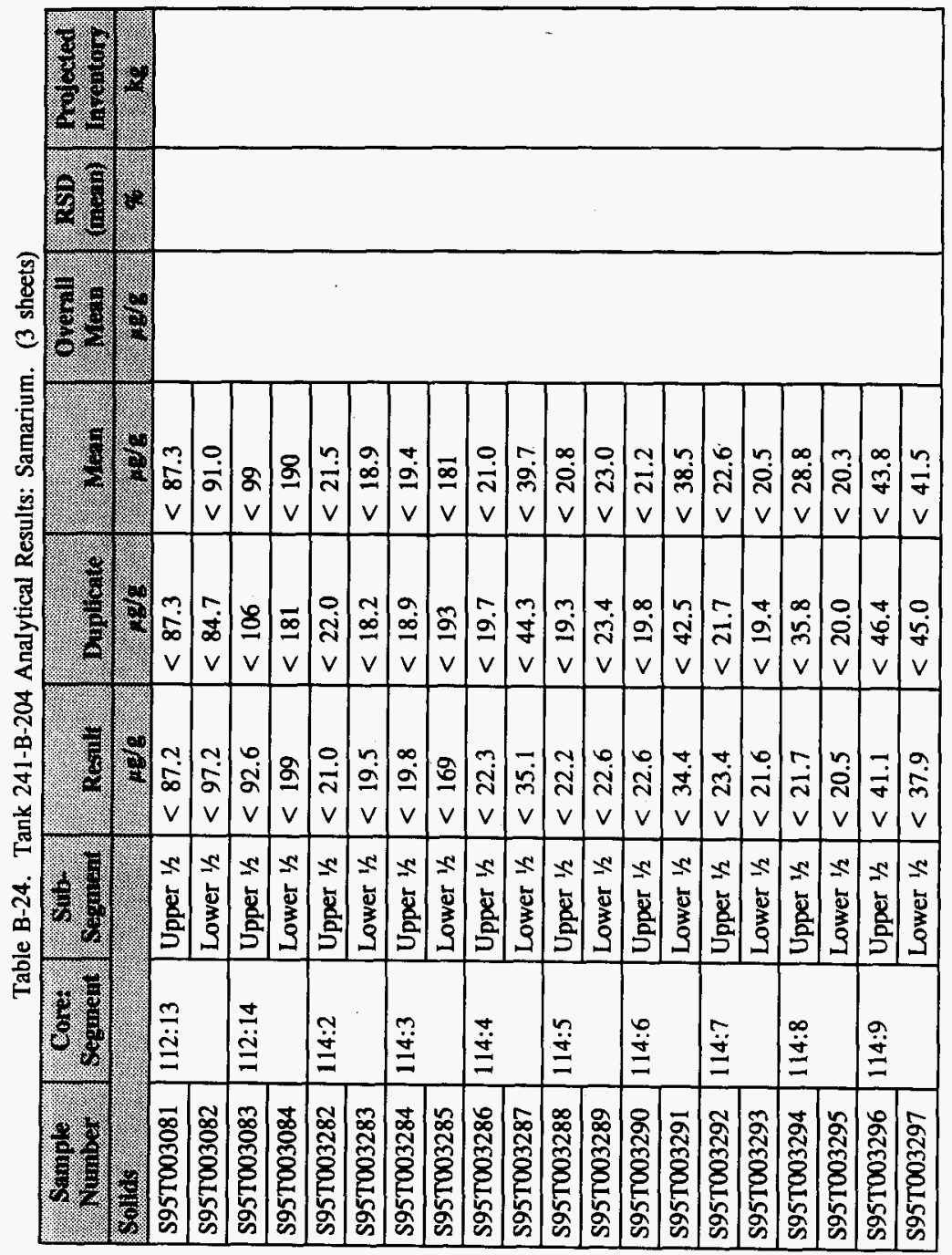


Table B-24. Tank 241-B-204 Analytical Results: Samarium. (3 sheets)

\begin{tabular}{|c|c|c|c|c|c|c|c|c|}
\hline Somplo & 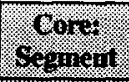 & 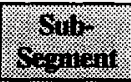 & 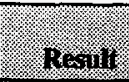 & $8,10 \%$ & $\sqrt{3}$ & 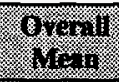 & 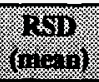 & how \\
\hline oilys, & & 8 & 28 & 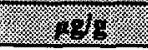 & (1) & (2) & , & (3) \\
\hline S95T003298 & \multirow[t]{2}{*}{$114: 10$} & Upper $1 / 2$ & $<37.0$ & $<35.9$ & $<36.5$ & & & \\
\hline S95T003299 & & Lower $1 / 2$ & $<33.9$ & $<38.5$ & $<36.7$ & & & \\
\hline$\$ 951003300$ & \multirow[t]{2}{*}{$114: 11$} & Upper $1 / 2$ & $<47.0$ & $<48.3$ & $<47.7$ & & & \\
\hline S95T003301 & & Lower $1 / 2$ & $<34.0$ & $<34.0$ & $<34.0$ & & & \\
\hline \$95T003302 & \multirow[t]{2}{*}{$114: 12$} & Upper $1 / 2$ & $<24.0$ & $<22.1$ & $<23.1$ & & & \\
\hline S95T003303 & & Lower $1 / 2$ & $<22.3$ & $<37.0$ & $<29.7$ & & & \\
\hline \$95T003304 & \multirow[t]{2}{*}{$114: 13$} & Upper $1 / 2$ & $<45.8$ & $<40.9$ & $<43.4$ & & & \\
\hline \$95T003305 & & Lower $1 / 2$ & $<36.0$ & $<36.6$ & $<36.3$ & & & \\
\hline S95T003306 & \multirow[t]{2}{*}{$114: 14$} & Upper $1 / 2$ & $<42.9$ & $<42.7$ & $<42.8$ & & & \\
\hline S95T003307 & & Lower $1 / 2$ & $<38.6$ & $<42.2$ & $<40.4$ & & & \\
\hline \multicolumn{3}{|c|}{ Dratioble hiulds. } & 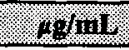 & Hority & Hint & $401 \mathrm{ni}$ & ( & 48 \\
\hline S95T002976 & 112:1 & $\mathrm{DL}$ & $<20.1$ & $<20.1$ & $<20.1$ & \multirow[t]{3}{*}{$<15.1$} & \multirow[t]{3}{*}{$n / a$} & \multirow[t]{3}{*}{$<0.0604$} \\
\hline S95T002984 & $112: 2$ & $\overline{D L}$ & $<20.1$ & $<20.1$ & $<20.1$ & & & \\
\hline S95T003255 & 114:1 & DL & $<10.1$ & $<10.1$ & $<10.1$ & & & \\
\hline
\end{tabular}


Table B-25. Tank 241-B-204 Analytical Results: Selenium. (3 sheets)

\begin{tabular}{|c|c|c|c|c|c|c|c|c|}
\hline Gonos & $\left(p_{0,1}\right)$ & $\log _{\log } \log _{311}$ & in & Buplis:3 & 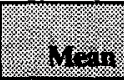 & \% & $\frac{101}{19}$ & 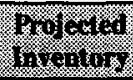 \\
\hline Sollins & 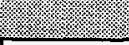 & & . & 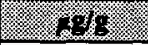 & 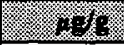 & 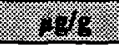 & (2) & 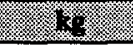 \\
\hline S95T002980 & $112: 2$ & Whole & $<27.6$ & $<37.1$ & $<32.4$ & \multirow[t]{21}{*}{$<44.9$} & \multirow[t]{21}{*}{$n / a$} & \multirow[t]{21}{*}{$<9.88$} \\
\hline S95T003061 & \multirow[t]{2}{*}{$112: 3$} & Upper $1 / 2$ & $<24.1$ & $<18.8$ & $<21.5$ & & & \\
\hline S95T003062 & & Lower $1 / 2$ & $<22.5$ & $<21.3$ & $<21.9$ & & & \\
\hline S95T003063 & \multirow[t]{2}{*}{$112: 4$} & Upper $1 / 2$ & $<17.6$ & $<23.7$ & $<20.7$ & & & \\
\hline S95T003064 & & Lower $1 / 2$ & $<24.3$ & 22.4 & 23.4 & & & \\
\hline S95T003065 & \multirow[t]{2}{*}{$112: 5$} & Upper $1 / 2$ & $<43.7$ & $<41.6$ & $<42.7$ & & & \\
\hline S95T003066 & & Lower $1 / 2$ & $<23.1$ & $<19.5$ & $<21.3$ & & & \\
\hline S95T003067 & \multirow[t]{2}{*}{$112: 6$} & Upper $1 / 2$ & $<46.6$ & $<34.6$ & $<40.6$ & & & \\
\hline S95T003068 & & Lower $1 / 2$ & $<22.3$ & 19.9 & 21.1 & & & \\
\hline S95T003069 & \multirow[t]{2}{*}{$112: 7$} & Upper $1 / 2$ & $<36.4$ & $<38.1$ & $<37.3$ & & & \\
\hline S95T003070 & & Lower $1 / 2$ & $<35.1$ & $<34.3$ & $<34.7$ & & & \\
\hline S95T003071 & \multirow[t]{2}{*}{$112: 8$} & Upper $1 / 2$ & $<21.0$ & 26.3 & 23.7 & & & \\
\hline S95T003072 & & Lower $1 / 2$ & $<39.1$ & $<38.1$ & $<38.6$ & & & \\
\hline \$95T003073 & \multirow[t]{2}{*}{$112: 9$} & Upper $1 / 2$ & $<41.4$ & $<38.0$ & $<39.6$ & & & \\
\hline S95T003074 & & Lower $1 / 2$ & $<34.3$ & $<38.1$ & $<36.2$ & & & \\
\hline S95T003075 & \multirow[t]{2}{*}{ 112:10 } & Upper $1 / 2$ & $<37.2$ & $<36.4$ & $<36.8$ & & & \\
\hline S95T003076 & & Lower $1 / 2$ & $<45.6$ & $<41.1$ & $<43.4$ & & & \\
\hline S95T003077 & \multirow[t]{2}{*}{ 112:11 } & Upper $1 / 2$ & $<40.7$ & $<34.8$ & $<37.7$ & & & \\
\hline S95T003078 & & Lower $1 / 2$ & $<94.9$ & $<115$ & $<105$ & & & \\
\hline S95T003079 & \multirow[t]{2}{*}{ 112:12 } & Upper $1 / 2$ & $<107$ & $<96.6$ & $<102$ & & & \\
\hline S95T003080 & & Lower $1 / 2$ & $<97.8$ & $<101$ & $<99$ & & & \\
\hline
\end{tabular}




\begin{tabular}{|c|c|c|c|c|c|c|c|c|}
\hline & & & $\varepsilon \cdot 2 t$ & $0 . s_{t}>$ & $09^{\circ} 6 \varepsilon$ & $z_{1}$ دәмот & \multirow[b]{2}{*}{$6: \mathrm{tII}$} & L6Zع00LS6S \\
\hline & & & $8^{\circ} \mathrm{Et}>$ & $t 9 t>$ & I.It $>$ & $z / 1$ sadd $\Omega$ & & 96ZE00.LS6S \\
\hline & & & $0 \varepsilon^{\prime} I Z$ & $0 S^{\prime} I Z$ & oI'IZ & $z_{1}$ LOMOT & \multirow[b]{2}{*}{$8: t I I$} & S62E00LS6S \\
\hline & & & $8.82>$ & $8 . S E>$ & $L I Z>$ & $z / \mathrm{I}$ Iədd $\Omega$ & & t62ع00LS6S \\
\hline & & & $\varepsilon^{\prime} I Z$ & $06^{\circ} 02$ & $9.12>$ & $2 / 1 \triangle \mathrm{NOO}$ & \multirow[b]{2}{*}{ L:tII } & E6ZE00LS6S \\
\hline & & & $9.22>$ & $L \cdot I Z>$ & $\nabla \cdot E Z>$ & $2 / 1$ sadd $\Omega$ & & Z6ZE00LS6S \\
\hline & & & $58 E>$ & $s \cdot z t>$ & $\nabla \forall \varepsilon>$ & Z/1 IOMOT & \multirow[b]{2}{*}{$9: t 11$} & I6ZE00LS6S \\
\hline & & & $\tau \cdot 1 z>$ & $8.61>$ & $9.22>$ & $z / 1 \operatorname{sadd}_{\Omega}$ & & 06ZE00LS6S \\
\hline & & & $0 \varepsilon \varepsilon>$ & $t \varepsilon \mathcal{E}>$ & $9.22>$ & $2 / 1$ samo & \multirow[b]{2}{*}{$s: t I I$} & $68 z \varepsilon 00 \mathrm{LS} 6 \mathrm{~S}$ \\
\hline & & & $8.02>$ & $\varepsilon^{\prime} 61>$ & $2.22>$ & $z / 1$ Iodd $n$ & & 88ZE00LS6S \\
\hline & & & $\angle 6 \varepsilon>$ & $\varepsilon \neq t>$ & I'SE > & $3 / \mathrm{d}$ دOMO & \multirow[b]{2}{*}{$t: t 1 I$} & L8ZE00IS6S \\
\hline & & & $0.12>$ & $L 6 \mathrm{I}>$ & $\varepsilon \cdot z z>$ & $z_{1} \operatorname{ladd}_{\Omega}$ & & 982E00LS6S \\
\hline & & & $181>$ & $\varepsilon 61>$ & $691>$ & $3 / 1$ دәMO & \multirow[b]{2}{*}{$\varepsilon: t I I$} & S8ZE00IS6S \\
\hline & & & $76 I>$ & $681>$ & $8.61>$ & $z_{1}$ sadd $n$ & & t8zع0aLS6S \\
\hline & & & $681>$ & $281>$ & $5.61>$ & $y / 1$ دMO & \multirow[b]{2}{*}{$2: t 11$} & E8ZE00LS6S \\
\hline & & & siz> & $0.22>$ & $0.12>$ & $z / 1$ sodd $\Pi$ & & $28 z \varepsilon 00 L S 6 S$ \\
\hline & & & $06 \mathrm{I}>$ & $181>$ & $661>$ & $2 / 10 \mathrm{MO}$ & \multirow[b]{2}{*}{$D I: Z I I$} & $780 \varepsilon 00 \mathrm{LS} 6 \mathrm{~S}$ \\
\hline & & & $\varepsilon 66>$ & $901>$ & $9.26>$ & $z / 1$ sodd $n$ & & E80ع00LS6S \\
\hline & & & $0.16>$ & $\angle \neq 8>$ & $2.26>$ & $z_{1}$ मамоТ & \multirow[b]{2}{*}{ EI:ZII } & $280 E 00 \perp S 6 S$ \\
\hline & & & $\varepsilon^{\prime} \angle 8>$ & $\varepsilon \angle 8>$ & $2.28>$ & $z / 1$ Jadd $\Omega$ & & $180 \varepsilon 00 \mathrm{JS} 6 \mathrm{~S}$ \\
\hline 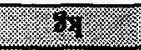 & 4 & VW & Th: & (67\% & Th: & (1) & (7) & $4314 \mathrm{~d}$ \\
\hline pringinis & $\frac{10 \%}{16 \%}$ & 1964\% & 4.4 & 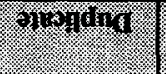 & 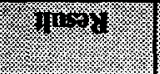 & 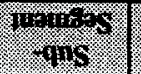 & 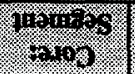 & 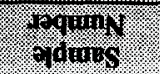 \\
\hline
\end{tabular}


Table B-25. Tank 241-B-204 Analytical Results: Selenium. (3 sheets)

\begin{tabular}{|c|c|c|c|c|c|c|c|c|}
\hline 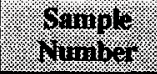 & 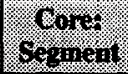 & 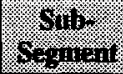 & Me-111 & $1314=10$ & $y=18$ & 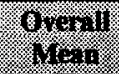 & $(2493$ & 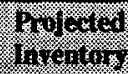 \\
\hline Solins & 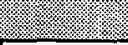 & 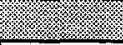 & 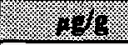 & 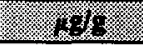 & 28 & 24 & 8 & 8 \\
\hline S95T003298 & \multirow[t]{2}{*}{$114: 10$} & Upper $1 / 2$ & 40.70 & 36.10 & 38.4 & & & \\
\hline S95T003299 & & Lower $1 / 2$ & $<33.9$ & $<38.5$ & $<36.2$ & & & \\
\hline S95T003300 & \multirow[t]{2}{*}{$114: 11$} & Upper $1 / 2$ & $<47.0$ & $<48.3$ & $<47.7$ & & & \\
\hline S95T003301 & & Lower $1 / 2$ & $<34.0$ & $<34.0$ & $<34.0$ & & & \\
\hline S95T003302 & \multirow[t]{2}{*}{$114: 12$} & Upper $1 / 2$ & $<24.0$ & $<22.1$ & $<23.1$ & & & \\
\hline \$95T003303 & & Lower $1 / 2$ & $<22.3$ & $<37.0$ & $<29.7$ & & & \\
\hline S95T003304 & \multirow[t]{2}{*}{$114: 13$} & Upper $1 / 2$ & $<45.8$ & $<40.9$ & $<43.4$ & & & \\
\hline \$95T003305 & & Lower $1 / 2$ & $<36.0$ & $<36.6$ & $<36.3$ & & & \\
\hline S95T003306 & \multirow[t]{2}{*}{$114: 14$} & Upper $1 / 2$ & $<42.9$ & $<42.7$ & $<42.8$ & & & \\
\hline S95T003307 & & Lower $1 / 2$ & $<38.6$ & $<42.2$ & $<40.4$ & & & \\
\hline \multicolumn{3}{|c|}{ Draninol Houlus } & 181111 & 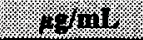 & 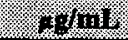 & 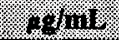 & ( & 13 \\
\hline S95T002976 & 112:1 & $\mathrm{DL}$ & $<20.1$ & $<20.1$ & $<20.1$ & \multirow[t]{3}{*}{$<15.1$} & \multirow[t]{3}{*}{$n / a$} & \multirow[t]{3}{*}{$<0.0604$} \\
\hline \$95T002984 & $112: 2$ & DL & $<20.1$ & $<20.1$ & $<20.1$ & & & \\
\hline S95T003255 & $114: 1$ & DL & $<10.1$ & $<10.1$ & $<10.1$ & & & \\
\hline
\end{tabular}




\begin{tabular}{|c|c|c|c|c|c|c|c|c|}
\hline Gompor & wow & 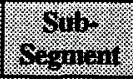 & Plowir & 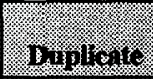 & 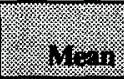 & (6) & 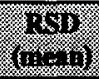 & Frofory \\
\hline oblos & (2) & (2) & 35 & $(8)$ & 48 & 40 & . & 8 \\
\hline S95T002980 & $112: 2$ & Whole & 2,170 & 2,090 & 2,130 & \multirow[t]{21}{*}{1,070} & \multirow[t]{21}{*}{22.8} & \multirow[t]{21}{*}{236} \\
\hline \$95T003061 & \multirow[t]{2}{*}{$112: 3$} & Upper $1 / 2$ & 1,120 & 1,400 & 1,260 & & & \\
\hline S95T003062 & & Lower $1 / 2$ & 1,420 & 1,470 & 1,440 & & & \\
\hline S95T003063 & \multirow[t]{2}{*}{$112: 4$} & Upper $1 / 2$ & 1,330 & 1,810 & 1,570 & & & \\
\hline S95T003064 & & Lower $1 / 2$ & 1,550 & 1,670 & 1,610 & & & \\
\hline \$95T003065 & \multirow[t]{2}{*}{$112: 5$} & Upper $1 / 2$ & 1,800 & 1,520 & 1,660 & & & \\
\hline S95T003066 & & Lower $1 / 2$ & 1,830 & 1,830 & 1,830 & & & \\
\hline \$95T003067 & \multirow[t]{2}{*}{$112: 6$} & Upper 1/2 & 1,770 & 1,970 & 1,870 & & & \\
\hline S95T003068 & & Lower $1 / 2$ & 1,770 & 1,570 & 1,670 & & & \\
\hline S95T003069 & \multirow[t]{2}{*}{$112: 7$} & Upper $1 / 2$ & 1,650 & 1,960 & 1,800 & & & \\
\hline \$95T003070 & & Lower $1 / 2$ & 1,790 & 1,680 & 1,740 & & & \\
\hline S95T003071 & \multirow[t]{2}{*}{$112: 8$} & Upper $1 / 2$ & 1,200 & 1,220 & 1,210 & & & \\
\hline S95T003072 & & Lower $1 / 2$ & 1,400 & 1,500 & 1,450 & & & \\
\hline S95T003073 & \multirow[t]{2}{*}{$112: 9$} & Upper $1 / 2$ & 1,290 & 1,490 & 1,390 & & & \\
\hline S95T003074 & & Lower $1 / 2$ & 1,370 & 1,440 & 1,400 & & & \\
\hline S95T003075 & \multirow[t]{2}{*}{$112: 10$} & Upper $1 / 2$ & 1,290 & 1,570 & 1,430 & & & \\
\hline S95T003076 & & Lower $1 / 2$ & 1,190 & 1,130 & 1,160 & & & \\
\hline \$95T003077 & \multirow[t]{2}{*}{$112: 11$} & Upper $1 / 2$ & 929.0 & 767.0 & 848.0 & & & \\
\hline \$95T003078 & & Lower $1 / 2$ & 598.0 & 504.0 & 551.0 & & & \\
\hline S95T003079 & \multirow[t]{2}{*}{$112: 12$} & Upper $1 / 2$ & 674.0 & 614.0 & 644.0 & & & \\
\hline$\$ 95 T 003080$ & & Lower $1 / 2$ & 788.0 & 511.0 & 649.5 & & & \\
\hline
\end{tabular}




\begin{tabular}{|c|c|c|c|c|c|c|c|c|}
\hline 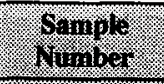 & 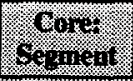 & $\frac{10}{40}$ & 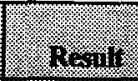 & 1 $13 y+101 \%$ & $8+18$ & to & $(1018$ & posporit. \\
\hline 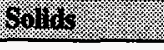 & (1. & & . & . & (r) & 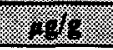 & (1. & 8 \\
\hline S95T003081 & \multirow[t]{2}{*}{$112: 13$} & Upper $1 / 2$ & 455.0 & 343.0 & 399.0 & & & \\
\hline S95T003082 & & Lower $1 / 2$ & 655.0 & 565.0 & 610.0 & & & \\
\hline S95T003083 & \multirow[t]{2}{*}{ 112:14 } & Upper $1 / 2$ & 589.0 & 582.0 & 585.5 & & & \\
\hline S95T003084 & & Lower $1 / 2$ & 1,200 & 959.0 & 1,080 & & & \\
\hline S95T003282 & \multirow[t]{2}{*}{$114: 2$} & Upper $1 / 2$ & 998.0 & 1,010 & 1,000 & & & \\
\hline S95T003283 & & Lower $1 / 2$ & 395.0 & 400.0 & 397.5 & & & \\
\hline S95T003284 & \multirow[t]{2}{*}{$114: 3$} & Upper $1 / 2$ & 444.0 & 368.0 & 406.0 & & & \\
\hline \$95T003285 & & Lower $1 / 2$ & 468.0 & 490.0 & 479.0 & & & \\
\hline S95T003286 & \multirow[t]{2}{*}{$114: 4$} & Upper $1 / 2$ & 642.0 & 1,150 & 896.0 & & & \\
\hline S95T003287 & & Lower $1 / 2$ & 1,220 & 905.0 & 1,060 & & & \\
\hline S95T003288 & \multirow[t]{2}{*}{$114: 5$} & Upper $1 / 2$ & 589.0 & 484.0 & 536.5 & & & \\
\hline S95T003289 & & Lower $1 / 2$ & 897.0 & 953.0 & 925.0 & & & \\
\hline$\$ 95 T 003290$ & \multirow[t]{2}{*}{$114: 6$} & Upper $1 / 2$ & 865.0 & 992.0 & 928.5 & & & \\
\hline S95T003291 & & Lower $1 / 2$ & 573.0 & 764.0 & 668.5 & & & \\
\hline S95T003292 & \multirow[t]{2}{*}{$114: 7$} & Upper $1 / 2$ & 521.0 & 1,070 & 795.5 & & & \\
\hline S95T003293 & & Lower $1 / 2$ & 810.0 & 566.0 & 688.0 & & & \\
\hline S95T003294 & \multirow[t]{2}{*}{$114: 8$} & Upper $1 / 2$ & 1,500 & 1,170 & 1,340 & & & \\
\hline S95T003295 & & Lower $1 / 2$ & 390.0 & 781.0 & 585.5 & & & \\
\hline S95T003296 & \multirow[t]{2}{*}{$114: 9$} & Upper $1 / 2$ & 364.0 & 897.0 & 630.5 & & & \\
\hline S95T003297 & & Lower $1 / 2$ & 833.0 & 898.0 & 865.5 & & & \\
\hline
\end{tabular}




\begin{tabular}{|c|c|c|c|c|c|c|c|c|}
\hline \multirow[b]{3}{*}{$t \nabla Z^{\prime} 0$} & \multirow[b]{3}{*}{$0.0 \mathrm{I}$} & \multirow[b]{3}{*}{6.09} & $\$ 6.99$ & 00.99 & $06 \div 9$ & 70 & $I: D I I$ & SSZE001S6S \\
\hline & & & 59.65 & $0 \varepsilon^{\prime} 6 S$ & 00.09 & 70 & Z:ZII & t86200LS6S \\
\hline & & & 50.05 & $00^{\circ} 0 \mathrm{~s}$ & $0<6 t$ & $\mathrm{TQ}$ & I:ZII & 9L6200LS6S \\
\hline (7) & 8 & mopt & $101 \%$ & Twifl: & ming & \multirow{2}{*}{$\frac{z_{1} \text { IəMOT }}{1}$} & \multicolumn{2}{|c|}{ 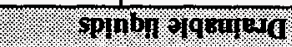 } \\
\hline & & & 0.518 & 0.218 & 0.818 & & \multirow[b]{2}{*}{$D I: t I I$} & LOEE00LS6S \\
\hline & & & S\$S8 & 0.268 & 0.618 & $z_{1} \operatorname{sodd}_{n}$ & & 90EE00LS6S \\
\hline & & & 0.788 & $0 .+06$ & 0.098 & $3 / 1$ IOMOOT & \multirow[b]{2}{*}{$\mathcal{E I}: t \boldsymbol{I I}$} & SOEEO0LS6S \\
\hline & & & $6.5+6$ & 0.096 & $0.6 \varepsilon 6$ & $y_{1} \operatorname{Iadd}_{\Omega}$ & & tOEEOOLS6S \\
\hline & & & $000^{\circ} \mathrm{I}$ & $0.9 \angle 6$ & $0 E 0^{\prime} \mathrm{I}$ & Z/1 गОМО & \multirow[b]{2}{*}{ ZI:tII } & E0EE00LS6S \\
\hline & & & 5.856 & $060^{\circ} \mathrm{I}$ & $0 . \angle 28$ & $z_{1}$ Iædd $n$ & & ZOEEOOLS6S \\
\hline & & & $0 E I^{\prime} I$ & $001^{\circ} \mathrm{l}$ & $0 Z I^{\prime} \mathrm{I}$ & $z / 1$ JOMOT & \multirow[b]{2}{*}{ II:DII } & IOEEOaLS6S \\
\hline & & & $0+0^{\prime} \mathrm{I}$ & $080^{\circ} \mathrm{I}$ & $0 \div 566$ & $z / 1$ Iadd $n$ & & 00EE00LS6S \\
\hline & & & S9L8 & 0.268 & 0.958 & $2 / 1$ JPMOT & \multirow[b]{2}{*}{ OI:DII } & 66ZE00IS6S \\
\hline & & & 0.029 & $00+9$ & 0.009 & $z / 1$ Iadd & & $86 Z E 00 \perp S 6 S$ \\
\hline 2 & 3 & (1) & $3 \%$ & (19\% & Wr & (4) & (1) & (196102 \\
\hline 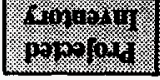 & 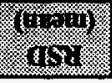 & 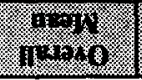 & 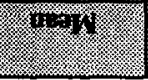 & (2) & mora & $\begin{array}{c}\text { Trums } \\
\text { quss }\end{array}$ & $\sqrt{90606}$ & $\begin{array}{l}\text { ragming } \\
\text { qurns }\end{array}$ \\
\hline
\end{tabular}


Table B-27. Tank 241-B-204 Analytical Results: Silver. (3 sheets)

\begin{tabular}{|c|c|c|c|c|c|c|c|c|}
\hline Whoplo & 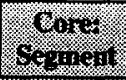 & 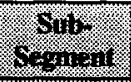 & $(\mathrm{p}(\mathrm{s}, 1)$ & Hinglong & $31=3$ & $6 \%$ & 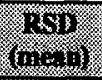 & 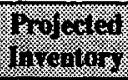 \\
\hline Sollo & & & 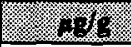 & 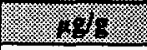 & 89 & 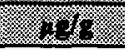 & (1., & 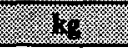 \\
\hline S95T002980 & $112: 2$ & Whole & 8.070 & 7.600 & 7.835 & \multirow[t]{21}{*}{$<5.56$} & \multirow[t]{21}{*}{$n / a$} & \multirow[t]{21}{*}{$<1.22$} \\
\hline S95T003061 & \multirow[t]{2}{*}{$112: 3$} & Upper $1 / 2$ & 3.070 & 2.940 & 3.005 & & & \\
\hline S95T003062 & & Lower $1 / 2$ & 3.460 & 3.270 & 3.365 & & & \\
\hline S95T003063 & \multirow[t]{2}{*}{$112: 4$} & Upper $1 / 2$ & 2.120 & 2.800 & 2.460 & & & \\
\hline S95T003064 & & Lower $1 / 2$ & 3.970 & 3.750 & 3.860 & & & \\
\hline S95T003065 & \multirow[t]{2}{*}{$112: 5$} & Upper $1 / 2$ & $<4.37$ & $<4.16$ & $<4.27$ & & & \\
\hline \$95T003066 & & Lower $1 / 2$ & 4.170 & 3.740 & 3.955 & & & \\
\hline \$95T003067 & \multirow[t]{2}{*}{$112: 6$} & Upper $1 / 2$ & $<4.66$ & 3.660 & 4.16 & & & \\
\hline \$95T003068 & & Lower $1 / 2$ & 3.980 & 3.140 & 3.560 & & & \\
\hline S95T003069 & \multirow[t]{2}{*}{$112: 7$} & Upper $1 / 2$ & $<3.640$ & 3.850 & 3.75 & & & \\
\hline \$95T003070 & & Lower $1 / 2$ & 4.170 & 4.010 & 4.090 & & & \\
\hline S95T003071 & \multirow[t]{2}{*}{$112: 8$} & Upper $1 / 2$ & 4.010 & 3.680 & 3.845 & & & \\
\hline S95T003072 & & Lower $1 / 2$ & 4.450 & 4.670 & 4.560 & & & \\
\hline S95T003073 & \multirow[t]{2}{*}{$112: 9$} & Upper $1 / 2$ & 4.990 & 4.460 & 4.725 & & & \\
\hline S95T003074 & & Lower $1 / 2$ & 3.870 & 4.190 & 4.030 & & & \\
\hline S95T003075 & \multirow[t]{2}{*}{$112: 10$} & Upper $1 / 2$ & 3.730 & $<3.64$ & 3.69 & & & \\
\hline S95T003076 & & Lower $1 / 2$ & 4.730 & 4.480 & 4.605 & & & \\
\hline S95T003077 & \multirow[t]{2}{*}{ 112:11 } & Upper $1 / 2$ & $<4.07$ & $<3.48$ & $<3.78$ & & & \\
\hline S95T003078 & & Lower $1 / 2$ & $<9.49$ & $<11.5$ & $<10.5$ & & & \\
\hline S95T003079 & \multirow[t]{2}{*}{$112: 12$} & Upper $1 / 2$ & $<10.7$ & $<9.66$ & $<10.2$ & & & \\
\hline S95T003080 & & Lower $1 / 2$ & $<9.78$ & $<10.1$ & $<9.94$ & & & \\
\hline
\end{tabular}


Table B-27. Tank 241-B-204 Analytical Resuits: Silver. (3 sheets)

\begin{tabular}{|c|c|c|c|c|c|c|c|c|}
\hline Miniole & orotion & 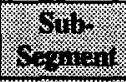 & r. & 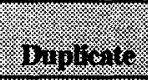 & S & $4 \%$ & 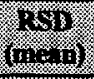 & $3 \%$ \\
\hline Solts & 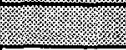 & & 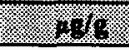 & $\times 8$ & 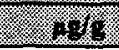 & \%) & (1) & (2) \\
\hline S95T003081 & \multirow[t]{2}{*}{$112: 13$} & Upper $1 / 2$ & $<8.72$ & $<8.73$ & $<8.73$ & & & \\
\hline S95T003082 & & Lower $1 / 2$ & $<9.72$ & $<8.47$ & $<9.10$ & & & \\
\hline \$95T003083 & \multirow[t]{2}{*}{$112: 14$} & Upper $1 / 2$ & $<9.26$ & $<10.6$ & $<9.93$ & & & \\
\hline S95T003084 & & Lower $1 / 2$ & $<19.9$ & $<18.1$ & $<19.0$ & & & \\
\hline S95T003282 & \multirow[t]{2}{*}{$114: 2$} & Upper $1 / 2$ & $<2.10$ & $<2.20$ & $<2.15$ & & & \\
\hline S95T003283 & & Lower $1 / 2$ & $<1.95$ & $<1.82$ & $<18.9$ & & & \\
\hline S95T003284 & \multirow[t]{2}{*}{$114: 3$} & Upper $1 / 2$ & $<1.98$ & $<1.89$ & $<1.94$ & & & \\
\hline S95T003285 & & Lower $1 / 2$ & $<16.9$ & $<19.3$ & $<18.1$ & & & \\
\hline S95T003286 & \multirow[t]{2}{*}{$114: 4$} & Upper $1 / 2$ & 2.240 & $<1.97$ & 2.11 & & & \\
\hline S95T003287 & & Lower $1 / 2$ & $<3.51$ & $<4.43$ & $<3.97$ & & & \\
\hline S95T003288 & \multirow[t]{2}{*}{$114: 5$} & Upper $1 / 2$ & $<2.22$ & $<1.93$ & $<2.08$ & & & \\
\hline S95T003289 & & Lower $1 / 2$ & $<2.26$ & $<2.34$ & $<2.30$ & & & \\
\hline S95T003290 & \multirow[t]{2}{*}{$114: 6$} & Upper $1 / 2$ & $<2.26$ & $<1.98$ & $<2.12$ & & & \\
\hline \$95T003291 & & Lower $1 / 2$ & $<3.44$ & $<4.25$ & $<3.85$ & & & \\
\hline S95T003292 & \multirow[t]{2}{*}{$114: 7$} & Upper $1 / 2$ & $<2.34$ & $<2.17$ & $<2.26$ & & & \\
\hline S95T003293 & & Lower $1 / 2$ & $<2.16$ & $<1.94$ & $<2.05$ & & & \\
\hline S95T003294 & \multirow[t]{2}{*}{$114: 8$} & Upper $1 / 2$ & $<2.17$ & $<3.58$ & $<2.88$ & & & \\
\hline S95T003295 & & Lower $1 / 2$ & 18.70 & 3.850 & 11.28 & & & \\
\hline S95T003296 & \multirow[t]{2}{*}{$114: 9$} & Upper $1 / 2$ & $<4.11$ & $<4.64$ & $<4.38$ & & & \\
\hline S95T003297 & & Lower $1 / 2$ & $<3.79$ & $<4.50$ & $<4.15$ & & & \\
\hline
\end{tabular}


Table B-27. Tank 241-B-204 Analytical Results: Silver. (3 sheets)

\begin{tabular}{|c|c|c|c|c|c|c|c|c|}
\hline Manpl & orotion & 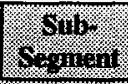 & Wo.nill. & 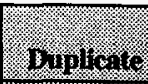 & $3 y=n$ & $\frac{1}{64}$ & 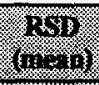 & 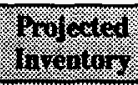 \\
\hline Sollis: & ? & (x) & 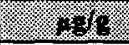 & 8 & 10 & 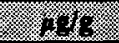 & 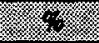 & (3) \\
\hline S95T003298 & \multirow[t]{2}{*}{$114: 10$} & Upper $1 / 2$ & $<3.70$ & $<3.59$ & $<3.65$ & & & \\
\hline \$95T003299 & & Lower $1 / 2$ & 5.450 & 5.020 & 5.235 & & & \\
\hline S95T003300 & \multirow[t]{2}{*}{$114: 11$} & Upper $1 / 2$ & 5.080 & $<4.83$ & 4.96 & & & \\
\hline S95T003301 & & Lower $1 / 2$ & $4.280^{\circ}$ & 4.320 & 4.300 & & & \\
\hline S95T003302 & \multirow[t]{2}{*}{$114: 12$} & Upper $1 / 2$ & 3.960 & 4.130 & 4.045 & & & \\
\hline S95T003303 & & Lower $1 / 2$ & 4.460 & 4.630 & 4.545 & & & \\
\hline \$95T003304 & \multirow[t]{2}{*}{$114: 13$} & Upper $1 / 2$ & 5.810 & 5.780 & 5.795 & & & \\
\hline S95T003305 & & Lower $1 / 2$ & 4.530 & 4.760 & 4.645 & & & \\
\hline S95T003306 & \multirow[t]{2}{*}{$114: 14$} & Upper $1 / 2$ & 4.760 & 4.410 & 4.585 & & & \\
\hline S95T003307 & & Lower $1 / 2$ & 4.300 & 4.340 & 4.320 & & & \\
\hline \multicolumn{3}{|c|}{ Bramble lourts } & ag/nil & irint & $42 / \mathrm{nL}$ & . $4 \mathrm{dmin}$ & 9 & (6) \\
\hline S95T002976 & 112:1 & DL & $<2.01$ & $<2.01$ & $<2.01$ & \multirow[t]{3}{*}{$<1.51$} & \multirow[t]{3}{*}{$\mathrm{n} / \mathrm{a}$} & \multirow[t]{3}{*}{$<0.00604$} \\
\hline S95T002984 & 112:2 & DL & $<2.01$ & $<2.01$ & $<2.01$ & & & \\
\hline S95T003255 & $114: 1$ & $\mathrm{DL}$ & $<1.01$ & $<1.01$ & $<1.01$ & & & \\
\hline
\end{tabular}




\begin{tabular}{|c|c|c|c|c|c|c|c|c|}
\hline & & able B-28. T & Tank 241-B & 4 Analytica & esults: & 1. (3 sheets) & & \\
\hline andinger & 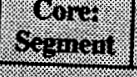 & aringing & 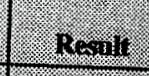 & (y) & 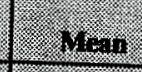 & 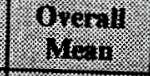 & $\frac{1+13}{(10 \%)}$ & 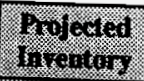 \\
\hline Oolins & & & 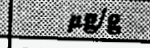 & 28 & $28 b_{3}$ & 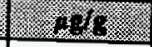 & (3) & 18 \\
\hline S95T002980 & $112: 2$ & Whole & 21,900 & 23,200 & 22,600 & \multirow{21}{*}{26,200} & \multirow[t]{21}{*}{2.3} & \multirow[t]{21}{*}{5,770} \\
\hline S95T003061 & \multirow[t]{2}{*}{$112: 3$} & Upper $1 / 2$ & 22,700 & 22,100 & 22,400 & & & \\
\hline S95T003062 & & Lower $1 / 2$ & 23,200 & 23,300 & 23,200 & & & \\
\hline S95T003063 & \multirow[t]{2}{*}{$112: 4$} & Upper $1 / 2$ & 20,500 & 27,000 & 23,800 & & & \\
\hline S95T003064 & & Lower $1 / 2$ & 24,900 & 24,800 & 24,800 & & & \\
\hline S95T003065 & \multirow[t]{2}{*}{$112: 5$} & Upper $1 / 2$ & 26,000 & 22,600 & 24,300 & & & \\
\hline S95T003066 & & Lower $1 / 2$ & 23,800 & 23,300 & 23,600 & & & \\
\hline \$95T003067 & \multirow[t]{2}{*}{$112: 6$} & Upper $1 / 2$ & 24,700 & 25,000 & 24,800 & & & \\
\hline \$95T003068 & & Lower $1 / 2$ & 26,600 & 23,300 & 25,000 & & & \\
\hline \$95T003069 & \multirow[t]{2}{*}{$112: 7$} & Upper $1 / 2$ & 23,500 & 25,800 & 24,600 & & & \\
\hline \$95T003070 & & Lower $1 / 2$ & 28,900 & 27,800 & 28,400 & & & \\
\hline s95T003071 & \multirow[t]{2}{*}{$112: 8$} & Upper $1 / 2$ & 24,000 & 24,000 & 24,000 & & & \\
\hline S95'r003072 & & Lower $1 / 2$ & 27,100 & 27,300 & 27,200 & & & \\
\hline S95T003073 & \multirow[t]{2}{*}{$112: 9$} & Upper $1 / 2$ & 26,800 & 25,900 & 26,400 & & & \\
\hline S95T003074 & & Lower $1 / 2$ & 28,100 & 26,600 & 27,400 & & & \\
\hline S95T003075 & \multirow[t]{2}{*}{$112: 10$} & Upper $1 / 2$ & 23,100 & 24,000 & 23,600 & & & \\
\hline \$95T003076 & & Lower $1 / 2$ & 25,700 & 26,100 & 25,900 & & & \\
\hline \$95T003077 & \multirow[t]{2}{*}{ 112:11 } & Upper $1 / 2$ & 25,300 & 25,900 & 25,600 & & & \\
\hline S95T003078 & & Lower $1 / 2$ & 27,800 & 27,800 & 27,800 & & & \\
\hline S95T003079 & \multirow[t]{2}{*}{$112: 12$} & Upper $1 / 2$ & 27,100 & 28,800 & 28,000 & & & \\
\hline S95T003080 & & Lower $1 / 2$ & 27,000 & 25,600 & 26,300 & & & \\
\hline
\end{tabular}




\begin{tabular}{|c|c|c|c|c|c|c|c|c|}
\hline & & & $00 t^{6} 82$ & $00 I^{\prime} 8 Z$ & $008^{\circ} 82$ & $\psi / 1$ IәмOT & \multirow[b]{2}{*}{$6: t I I$} & $\angle 6 Z E 00 \mathrm{~L} S 6 \mathrm{~S}$ \\
\hline & & & $00 L^{\prime} 8 Z$ & $00 I^{\prime} 82$ & $00 \varepsilon^{\prime} 6 z$ & $z_{1}$ Iodd $n$ & & 962E00LS6S \\
\hline & & & $00 z^{\prime} 92$ & $008^{6} \mathrm{~s}$ & $00 L^{\prime} 92$ & $z / 1$ JәMO & \multirow[b]{2}{*}{$8: t$ II } & S6Z200LS6S \\
\hline & & & $008^{\prime} \angle 2$ & $00 t^{\prime} 82$ & $00 I^{\prime} \angle Z$ & $z / 1$ dadd $\Omega$ & & $762 \varepsilon 00 \perp S 6 S$ \\
\hline & & & $000^{\prime} 62$ & $00 S^{\prime} \angle Z$ & $00 t^{\prime} 0 \varepsilon$ & $\psi_{1}$ Іәм07 & \multirow[b]{2}{*}{$L: D I I$} & E6ZE00LS6S \\
\hline & & & $00 z^{\prime} 9 z$ & $000^{\circ} \angle Z$ & $00 s^{\prime} s z$ & $z / 1 \operatorname{sodd} \Omega$ & & 262ع00LS6S \\
\hline & & & $00 z^{6} \angle z$ & $00 \varepsilon^{\prime} \angle Z$ & $001^{\circ} \angle 2$ & $z / 1$ IOMO & \multirow[b]{2}{*}{$9: t 11$} & I6Zع00LS6S \\
\hline & & & $009^{6} 82$ & $006^{\prime} \angle 2$ & $00 \varepsilon^{\prime} 6 z$ & $z / 1$ sadd $\Omega$ & & 06ZE00LS6S \\
\hline & & & $00 L^{\circ} \angle Z$ & $00 I^{\circ} \angle 2$ & $00 \varepsilon^{\prime} 8 Z$ & $z_{1}$ Іәмо & \multirow[b]{2}{*}{$s: t I l$} & 68ZE00LLGS \\
\hline & & & $005^{\prime} 92$ & $008^{\prime} \mathrm{sZ}$ & $00 z^{\prime} \angle z$ & $t_{1} \operatorname{Iodd}_{\Omega}$ & & $88 z \varepsilon 00 \mathrm{LS} 6 \mathrm{~S}$ \\
\hline & & & $008^{4} 92$ & $008^{\circ} 92$ & $008^{\circ} 92$ & $z / 1$ เәмо & \multirow[b]{2}{*}{$\nabla: t i l$} & L8zE00LS6S \\
\hline & & & $008^{\prime} \varepsilon z$ & $008^{6} \mathrm{GZ}$ & $008^{\prime} \varepsilon z$ & $3 / 1 \operatorname{sadd} \Omega$ & & $982 \varepsilon 00 \mathrm{LS6S}$ \\
\hline & & & $00 t^{\prime} s z$ & $00 L^{\prime} S Z$ & $00 I^{\prime} \mathrm{SZ}$ & $z / 1$ IOMO & \multirow[b]{2}{*}{$\varepsilon: t I I$} & S8ZE00LS6S \\
\hline & & & $009^{\prime}+2$ & $006^{6} \triangleright z$ & $00 t^{\circ} \nabla z$ & $4 / 1$ jedd $\Omega$ & & $78 Z \mathbb{E} 00 \mathrm{LS6S}$ \\
\hline & & & $00 t^{\circ}+2$ & $009^{4}+2$ & $00 z^{6} \nabla z$ & $2 / 12 \mathrm{MO}$ & \multirow[b]{2}{*}{$z: \pm 11$} & $\varepsilon 8 z \varepsilon 00 \mathrm{LS6S}$ \\
\hline & & & $008^{\circ}+2$ & $00 s^{\prime}+z$ & $000^{6} \mathrm{SZ}$ & $z / 1$ raddn & & z8ZE00LS6S \\
\hline & & & $000^{6} 62$ & $00 I^{\prime} 82$ & $000^{6} 0 E$ & $4 / 12 \mathrm{MO} 7$ & \multirow[b]{2}{*}{ tI:ZII } & $780 \mathrm{E} 00 \mathrm{LS} 6 \mathrm{~S}$ \\
\hline & & & $008^{\prime} 82$ & $00 L^{\prime} \angle Z$ & $006^{6} 62$ & $2 / 1$ Ladd $\Omega$ & & $\varepsilon 80 \varepsilon 00 \mathrm{LS} 6 \mathrm{~S}$ \\
\hline & & & $006^{6} 8 z$ & $00 t^{6} 82$ & $00 t^{6} 62$ & z/1 1әMO & \multirow[b]{2}{*}{$\mathcal{E}[$ ¿ZII } & $280 E 00 \mathrm{~L} S 6 \mathrm{~S}$ \\
\hline & & & $009^{6} 92$ & $00 L^{\prime} 9 Z$ & $00 t^{6} 9 z$ & $z_{1}$ Iəddn & & I80E00LS6S \\
\hline (1) & 4. & Whe & (WF & 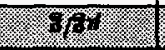 & Wort & (1) & 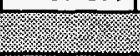 & splas \\
\hline 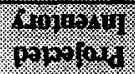 & (198\%) & 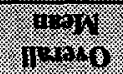 & I. & 076011190 & 119.9? & leomotion & 11100106\% & 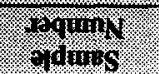 \\
\hline
\end{tabular}


Table B-28. Tank 241-B-204 Analytical Results: Sodium. (3 sheets)

\begin{tabular}{|c|c|c|c|c|c|c|c|c|}
\hline Ginily & 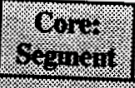 & $\frac{0}{011 \%}$ & isonil. & $3,101,243$ & m. & $6 \%$ & $(1,4)$ & 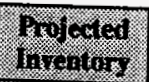 \\
\hline Woly & & & 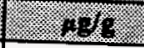 & 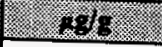 & 82 & 210 & \% & (3) \\
\hline S95T003298 & \multirow[t]{2}{*}{$114: 10$} & Upper $1 / 2$ & 29,600 & 28,300 & 29,000 & & & \\
\hline S95T003299 & & Lower $1 / 2$ & 27,200 & 27,400 & 27,300 & & & \\
\hline \$95T003300 & \multirow[t]{2}{*}{$114: 11$} & Upper $1 / 2$ & 28,000 & 27,500 & 27,800 & & & \\
\hline S95T003301 & & Lower $1 / 2$ & 29,400 & 27,900 & 28,600 & & & \\
\hline S95T003302 & \multirow[t]{2}{*}{$114: 12$} & Upper $1 / 2$ & 26,500 & 27,200 & 26,800 & & & \\
\hline S95T003303 & & Lower $1 / 2$ & 27,400 & 27,900 & 27,600 & & & \\
\hline \$95T003304 & \multirow[t]{2}{*}{$114: 13$} & Upper $1 / 2$ & 29,100 & 28,600 & 28,800 & & & \\
\hline S95T003305 & & Lower $1 / 2$ & 25,600 & 25,900 & 25,800 & & & \\
\hline S95T003306 & \multirow[t]{2}{*}{$114: 14$} & Upper $1 / 2$ & 25,100 & 25,600 & 25,400 & & & \\
\hline \$95T003307 & & Lower $1 / 2$ & 23,500 & 25,000 & 24,200 & & & \\
\hline \multicolumn{3}{|c|}{ 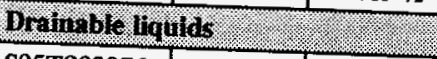 } & .9.1118 & 20, & (11) & 401111 & 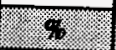 & 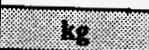 \\
\hline S95T002976 & 112:1 & DL & 28,400 & 28,300 & 28,400 & \multirow[t]{3}{*}{28,000} & \multirow{3}{*}{2.2} & \multirow[t]{3}{*}{112} \\
\hline S95T002984 & $112: 2$ & $\mathrm{DL}$ & 26,800 & 26,400 & 26,600 & & & \\
\hline S95T003255 & $114: 1$ & $\mathrm{DL}$ & 28,900 & 28,100 & 28,500 & & & \\
\hline
\end{tabular}




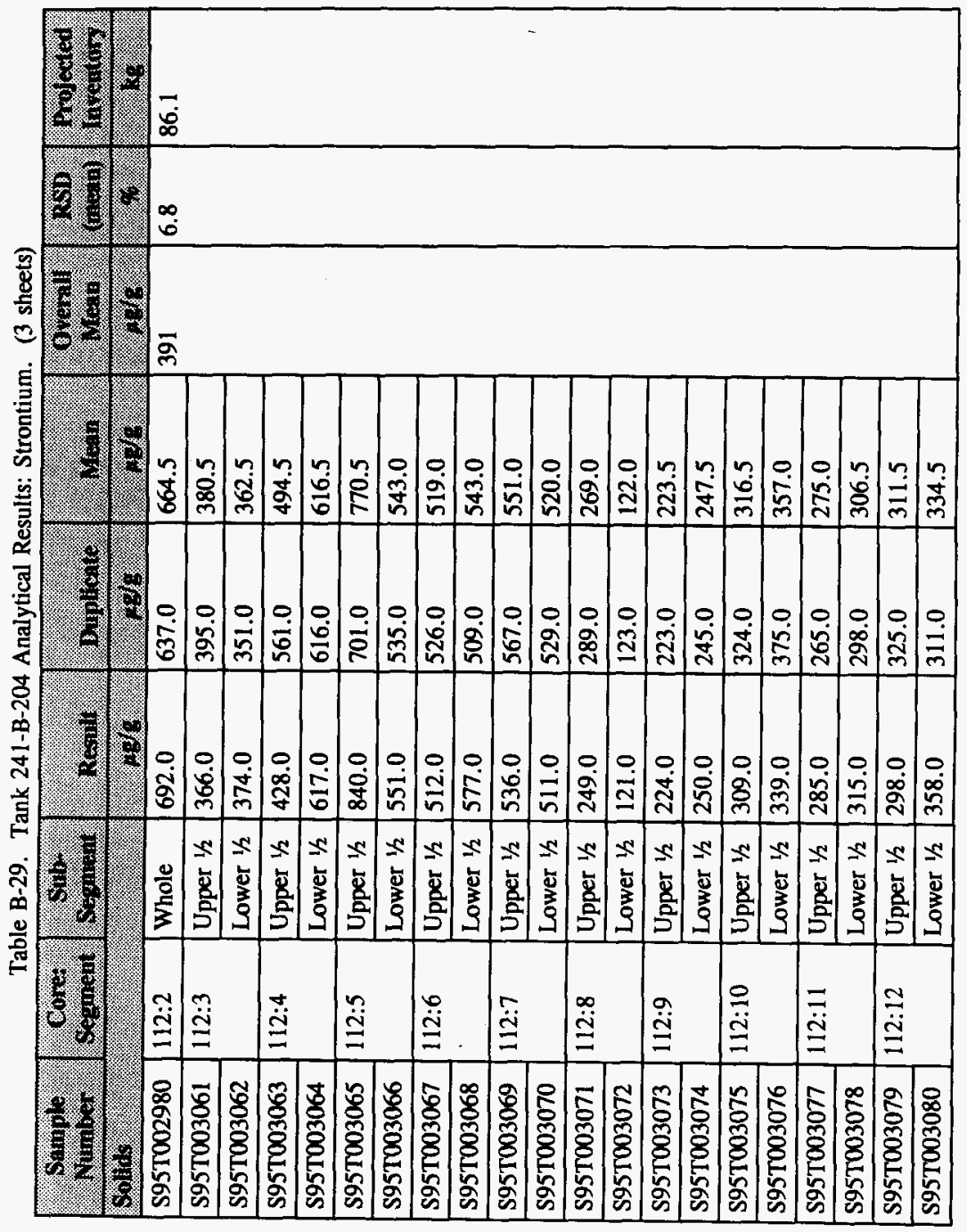




\begin{tabular}{|c|c|c|c|c|c|c|c|c|}
\hline & & & 5.582 & 0.262 & $0.62 Z$ & $2 / 1$ Iәмо & \multirow[b]{2}{*}{$6: t I I$} & L6ZE00LS6S \\
\hline & & & $s z 2$ & $0.5 z z$ & 0.092 & $\psi / 1$ Iədd $\Omega$ & & 962E00LS6S \\
\hline & & & 0.601 & $0 . \mathrm{SOI}$ & $0^{\circ} \varepsilon I I$ & $4 / 1$ Iәмо & \multirow[b]{2}{*}{$8: 011$} & S6ZE00LS6S \\
\hline & & & 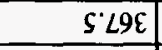 & $0.8 \angle \varepsilon$ & $0 . \angle S E$ & $z / 1$ Iadd $\Omega$ & & $762 \mathrm{E} 00 \perp 56 \mathrm{~S}$ \\
\hline & & & $0.90 \varepsilon$ & 0.162 & $0.12 \varepsilon$ & $2 / 1$ गОMOT & \multirow[b]{2}{*}{$L: t I I$} & E6ZE00LS6S \\
\hline & & & s'zzs & $0.2 t s$ & $0^{\circ} \varepsilon 0 S$ & $y_{1}$ Iadd $n$ & & 26zE00LS6S \\
\hline & & & $s .8+s$ & $0.5+5$ & $0.25 S$ & $4 / 1$ IOMOT & \multirow[b]{2}{*}{$9: t I I$} & I6ZE00IS6S \\
\hline & & & 0.915 & 0060 & $0 . z+s$ & $z / 1$ Iadd $\Omega$ & & 06ZE00LS6S \\
\hline & & & $s^{\circ}+\angle t$ & $0 \angle L t$ & $0^{\circ} Z \angle t$ & $4 / 1$ IOMO7 & \multirow[b]{2}{*}{ s:tII } & 68ZE00IS6S \\
\hline & & & 5929 & 0.209 & $0.9+9$ & $z / 1$ Iədd $\Omega$ & & $88 z E 00 \mathrm{LS} 6 \mathrm{~S}$ \\
\hline & & & $S^{*}$ tE9 & 0.869 & 0.129 & $y_{1}$ I2MOT & \multirow[b]{2}{*}{$t: t I I$} & L8z\&00LS6S \\
\hline & & & 0.015 & 0.115 & $0.60 \mathrm{~S}$ & $z / 1$ Iədd & & 98ZE00LS6S \\
\hline & & & $s+s t$ & $0 \rightarrow S t$ & $0 . s s t$ & \%/ IOMO & \multirow[b]{2}{*}{$\mathcal{E}: t I I$} & S8ZE00LS6S \\
\hline & & & S.8IE & $0 \div Z \mathcal{E}$ & $0^{\circ} \mathrm{EIE}$ & $z_{1}$ Iəddn & & $78 z \varepsilon 00$ LS6S \\
\hline & & & S. $6 L E$ & $0 \div 8 \varepsilon$ & $0 . S \angle E$ & $z_{1}$ гәмо & \multirow[b]{2}{*}{$Z: t I I$} & E8ZE00LS6S \\
\hline & & & 0.2It & $0 \angle 00$ & OLLID & $z_{1}$ lodd $n$ & & 28zह0aLS6S \\
\hline & & & 0.662 & $0.1 \angle Z$ & $0 . \angle 2 E$ & $z_{1}$ IOMO & \multirow[b]{2}{*}{$01: 211$} & t80EOOLS6S \\
\hline & & & $0^{\circ} \mathrm{E} 0 \mathrm{t}$ & $0.9 \varepsilon \varepsilon$ & $0.0 \angle t$ & $z / 1$ radd $n$ & & E80E0aLS6S \\
\hline & & & $0.50 t$ & $0 . \angle 6 E$ & 0 IIt & $2 / 1$ dOMOT & \multirow[b]{2}{*}{$\varepsilon I: Z I J$} & $280 E 00 \mathrm{LS} 6 \mathrm{~S}$ \\
\hline & & & 0.212 & 0.612 & 0.502 & $4 / \mathrm{I}$ Idd $\Pi$ & & I80E00LS6S \\
\hline r. & 4 & (y) & 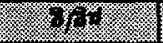 & $4 \%$ & (2) & (3. & (S) & . $9010 \mathrm{~S}$ \\
\hline 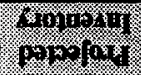 & 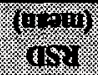 & 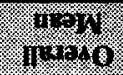 & $4.17 \%$ & oroforits & In? & 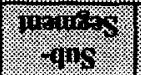 & 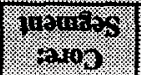 & 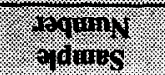 \\
\hline
\end{tabular}




\begin{tabular}{|c|c|c|c|c|c|c|c|c|}
\hline \multirow[b]{3}{*}{$\$ 0900.0>$} & \multirow[b]{3}{*}{$\mathrm{e} / \mathbf{u}$} & \multirow[b]{3}{*}{$I S^{\circ} I>$} & $10.1>$ & $10.1>$ & $10.1>$ & Ta & $I: t I I$ & SSZE00IS6S \\
\hline & & & $10 \cdot z>$ & $10^{\circ} z>$ & $10 \%>$ & 70 & $2: 211$ & $\$ 86200.156 \mathrm{~S}$ \\
\hline & & & $10 \cdot 2>$ & $10 \cdot 2>$ & $10 \%>$ & $\mathrm{Ta}$ & I:ZII & 9L6200LS6S \\
\hline 3 & 8. & 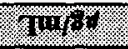 & nully & $140 \%=$ & THYr & gpingiquariva & \multicolumn{2}{|c|}{ 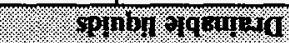 } \\
\hline & & & SIOE & $0.0 \varepsilon E$ & $0^{\circ} \varepsilon \angle Z$ & $2 / 1$ IอMOI & \multirow[b]{2}{*}{ tII:tII } & LOEEOOLS6S \\
\hline & & & S LZE & $0.62 \varepsilon$ & $0.92 \varepsilon$ & $z_{1}$ Iadd $n$ & & $90 E \varepsilon 00$ LS6S \\
\hline & & & S'OEE & $0+\varepsilon \varepsilon$ & $0 . \angle 2 \varepsilon$ & $2 / 1$ 12м07 & \multirow[b]{2}{*}{$\varepsilon I: \not I I$} & SOEE00.LS6S \\
\hline & & & 5.082 & 0.862 & $0 \_92$ & $z_{1}$ Iadd & & D0EE00IS6S \\
\hline & & & $5.82 z$ & $0.0 \varepsilon z$ & $0.22 z$ & $z_{1}$ IOMOT & \multirow[b]{2}{*}{ ZI:tII } & E0EE00LS6S \\
\hline & & & $s+t 0 z$ & 0.052 & $0.6 \varepsilon z$ & $4 / 1$ Iadd $n$ & & Z0EE00LS6S \\
\hline & & & S'ZEE & $0.82 \varepsilon$ & $0 . \angle \varepsilon \varepsilon$ & $z_{1}$ I2MO & \multirow[b]{2}{*}{ II:tII } & I0EE00LS6S \\
\hline & & & $0+62$ & $0^{\circ} \varepsilon S Z$ & $0 . S \angle Z$ & $z / 1$ sadd $\Omega$ & & DOEEDOLS6S \\
\hline & & & S.80t & O.IIt & $0.90 t$ & $2 / 1$ sәмо & \multirow[b]{2}{*}{$0 I: t I I$} & 662ع00LS6S \\
\hline & & & $S^{\circ} \varepsilon I Z$ & 0.012 & $0 \angle \mathrm{LI}$ & $z_{1}$ rodd $n$ & & 86ZE00LS6S \\
\hline (1) & 8 & (x) & (\% & $3 \mathrm{fH}$ & $7 \%$ & (2) & & 8 \\
\hline 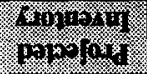 & 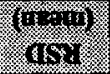 & 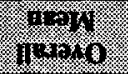 & 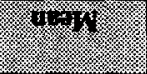 & 39? & 1110.96 & $\sqrt{1001060}$ & 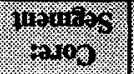 & ofoming \\
\hline
\end{tabular}

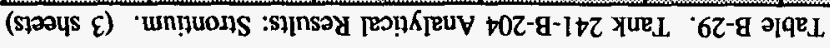




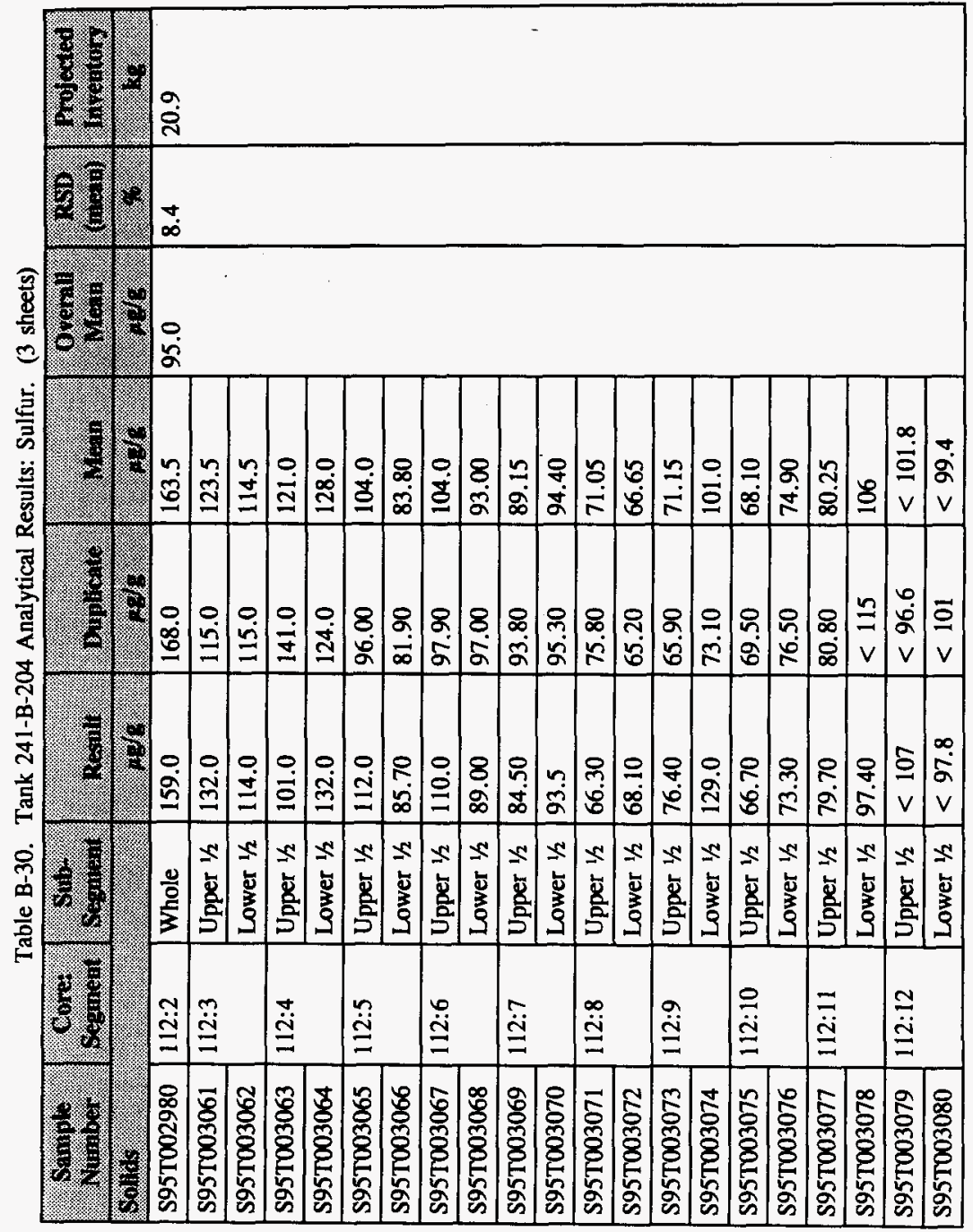




\begin{tabular}{|c|c|c|c|c|c|c|c|c|}
\hline & & & $0 I^{\circ} E L$ & OE IL & $06^{\circ}+L$ & $z_{1}$ دəMO二 & \multirow[b]{2}{*}{$6: t \mathrm{III}$} & $\angle 6 Z \varepsilon 00 L S 6 S$ \\
\hline & & & 0185 & 00.09 & $02^{\circ} 9 \mathrm{~s}$ & z/1 Ladd $\Pi$ & & 962E00LS6S \\
\hline & & & $\$ 9.29$ & 0199 & 02.65 & $3 / 1 \mathrm{~d} M \mathrm{MO}$ & \multirow[b]{2}{*}{$8: 511$} & S6ZE00LS6S \\
\hline & & & 0.001 & $0.0 E I$ & 0.051 & $z / 1$ Iədd $\Omega$ & & $+6 Z \varepsilon 00 L S 6 S$ \\
\hline & & & 06.19 & $0<95$ & $0 S^{\circ} \angle 9$ & $2 / 1$ ग्ल०ण & \multirow[b]{2}{*}{$L: t I I$} & E6ZE00LS6S \\
\hline & & & $59: 9 L$ & $02 \cdot 9 L$ & $0 I^{\circ} L L$ & z/1 Iadd $\Omega$ & & $26 Z \varepsilon 00 \mathrm{LS} 6 S$ \\
\hline & & & 06.08 & $00^{\circ} 58$ & $0 t^{\prime} 92$ & $z / 1$ dOMOT & \multirow[b]{2}{*}{$9: t I I$} & I6ZE00LS6S \\
\hline & & & $2 \cdot 101$ & $00^{\circ} \angle 8$ & 0 SII & $\mathcal{Y}_{\mathrm{l}}$ Iadd $\Omega$ & & 06ZE00LS6S \\
\hline & & & $8^{\circ} \mathrm{EOI}$ & 0.601 & $\angle 86$ & $2 / 1$ Iәмо & \multirow[b]{2}{*}{ s:til| } & 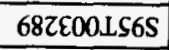 \\
\hline & & & 2.101 & $0 \varepsilon \$ 6$ & 0201 & $4 / 1$ Iədd $\Omega$ & & $88 Z E 00 L S 6 \mathrm{~S}$ \\
\hline & & & s.2ZI & $0 . \mathcal{E} \mathrm{I}$ & $0 \mathrm{ZII}$ & $4 / 12 M O 1$ & \multirow[b]{2}{*}{$t: t I I$} & $\angle 8 Z \varepsilon 00 \mathrm{LS} 6 \mathrm{~S}$ \\
\hline & & & $S \angle I I$ & $0+0 I I$ & $0 . \mathrm{IZI}$ & $z_{1}$ Iadd $_{\Omega}$ & & 982E00.LS6S \\
\hline & & & $181>$ & $E 61>$ & $691>$ & $y_{1}$ دәмо 1 & \multirow[b]{2}{*}{$\mathcal{E}: t I I$} & S8Zع00LS6S \\
\hline & & & S.SII & $0+t I I$ & $0.2 \mathrm{II}$ & $z / 1$ radd $\Omega$ & & $782800 . \mathrm{LS} 6 \mathrm{~S}$ \\
\hline & & & S. sII & 0.911 & $0^{\circ} \mathrm{sil}$ & $2 / 1$ गMOI & \multirow[b]{2}{*}{$Z: \Delta I I$} & E8ZE00.LS6S \\
\hline & & & 0.021 & 0.021 & $0.02 \mathrm{I}$ & $z / 12 \partial d_{n}$ & & 28ZE000LS6S \\
\hline & & & $06 \mathrm{I}>$ & I8I $>$ & $661>$ & $4 / 10 \mathrm{MOT}$ & \multirow[b]{2}{*}{ DI:ZII } & 780 E00.LS6S \\
\hline & & & $\varepsilon .66>$ & $90 \mathrm{I}>$ & $9.26>$ & $z / 1$ sadd $\Omega$ & & E80E00LS6S \\
\hline & & & $56.06>$ & $L+8>$ & $226>$ & $4 / \mathrm{I}$ IOMO $\mathrm{T}$ & \multirow[b]{2}{*}{ EI:ZII } & $280 \varepsilon 00, \mathrm{LS} 6 \mathrm{~S}$ \\
\hline & & & $S \mathcal{L} \angle 8>$ & $\varepsilon \angle 8>$ & $2 \angle 8>$ & $z / 1$ Iədd $\Omega$ & & I80E00.LS6S \\
\hline 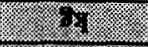 & (4) & 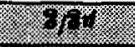 & $16 \%$ & 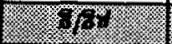 & fort: & & & GJ109 \\
\hline friponitit & (15-2.1) & $\begin{array}{l}91+14 \\
1+960\end{array}$ & 1011 & oforganging & yory & 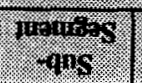 & 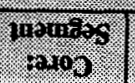 & 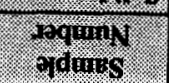 \\
\hline
\end{tabular}


Table B-30. Tank 241-B-204 Analytical Results: Sulfur. (3 sheets)

\begin{tabular}{|c|c|c|c|c|c|c|c|c|}
\hline Winge & 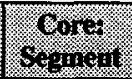 & ong & (18, & 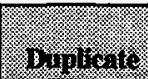 & $4 \%$ & 40 & 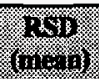 & 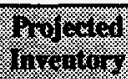 \\
\hline Solings & (2) & স্যে: & 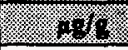 & 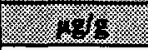 & (1) & $101 \%$ & . & (2) \\
\hline S95T003298 & \multirow[t]{2}{*}{ 114:10 } & Upper $1 / 2$ & 54.10 & 57.00 & 55.55 & & & \\
\hline S95T003299 & & Lower $1 / 2$ & 58.60 & 54.40 & 56.50 & & & \\
\hline$\$ 95 T 003300$ & \multirow[t]{2}{*}{$114: 11$} & Upper $1 / 2$ & 82.00 & 61.40 & 71.70 & & & \\
\hline \$95T003301 & & Lower $1 / 2$ & 64.90 & 53.60 & 59.25 & & & \\
\hline \$95T003302 & \multirow[t]{2}{*}{$114: 12$} & Upper $1 / 2$ & 64.40 & 66.10 & 65.25 & & & \\
\hline \$95T003303 & & Lower $1 / 2$ & 59.00 & 64.50 & 61.75 & & & \\
\hline S95T003304 & \multirow[t]{2}{*}{$114: 13$} & Upper $1 / 2$ & 79.50 & 60.50 & 70.00 & & & \\
\hline S95T003305 & & Lower $1 / 2$ & 55.50 & 62.90 & 59.20 & & & \\
\hline S95T003306 & \multirow[t]{2}{*}{$114: 14$} & Upper $1 / 2$ & 50.80 & 63.00 & 56.90 & & & \\
\hline S95T003307 & & Lower $1 / 2$ & 58.30 & 62.00 & 60.15 & & & \\
\hline \multicolumn{3}{|c|}{ Dolinqble jaulos } & 4.114 & 20/11\% & 491111 & $1 / 1113$ & $\%$ & 18 \\
\hline S95T002976 & $112: 1$ & DL & 135.0 & 132.0 & 133.5 & \multirow[t]{3}{*}{134} & \multirow[t]{3}{*}{3.7} & \multirow[t]{3}{*}{0.536} \\
\hline S95T002984 & $112: 2$ & DL & 125 & 122.0 & 123.5 & & & \\
\hline S95T003255 & $114: 1$ & DL & 142.0 & 135.0 & 138.5 & & & \\
\hline
\end{tabular}


Table B-31. Tank 241-B-204 Analytical Results: Thallium. (3 sheets)

\begin{tabular}{|c|c|c|c|c|c|c|c|c|}
\hline 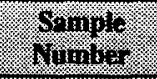 & 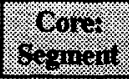 & (x) & $31=0.11$ & $1313 \times 10$ & $1 / 2=1$ & $4 \%$ & 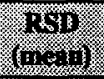 & 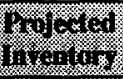 \\
\hline 00105 & (x) & 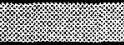 & 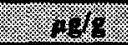 & $(25)=$ & 2015 & 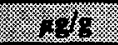 & 8 & 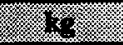 \\
\hline S95T002980 & $112: 2$ & Whole & $<55.3$ & $<74.3$ & $<64.8$ & \multirow[t]{21}{*}{$<89.6$} & \multirow[t]{21}{*}{$\mathrm{n} / \mathrm{a}$} & \multirow[t]{21}{*}{$<19.7$} \\
\hline S95T003061 & \multirow[t]{2}{*}{$112: 3$} & Upper $1 / 2$ & $<48.2$ & $<37.7$ & $<43.0$ & & & \\
\hline S95T003062 & & Lower $1 / 2$ & $<45.0$ & $<42.6$ & $<43.8$ & & & \\
\hline \$95T003063 & \multirow[t]{2}{*}{$112: 4$} & Upper $1 / 2$ & $<35.1$ & $<46.6$ & $<40.9$ & & & \\
\hline S95T003064 & & Lower $1 / 2$ & $<48.5$ & $<44.4$ & $<46.5$ & & & \\
\hline S95T003065 & \multirow[t]{2}{*}{$112: 5$} & Upper $1 / 2$ & $<87.4$ & $<83.2$ & $<85.3$ & & & \\
\hline \$95T003066 & & Lower $1 / 2$ & $<46.1$ & $<39.1$ & $<42.6$ & & & \\
\hline S95T003067 & \multirow[t]{2}{*}{$112: 6$} & Upper $1 / 2$ & $<93.3$ & $<68.9$ & $<81.1$ & & & \\
\hline S95T003068 & & Lower $1 / 2$ & $<44.5$ & $<38.7$ & $<41.6$ & & & \\
\hline S95T003069 & \multirow[t]{2}{*}{$112: 7$} & Upper $1 / 2$ & $<73.0$ & $<76.2$ & $<74.6$ & & & \\
\hline S95T003070 & & Lower $1 / 2$ & $<70.3$ & $<68.5$ & $<69.4$ & & & \\
\hline \$95T003071 & \multirow[t]{2}{*}{$112: 8$} & Upper $1 / 2$ & $<41.9$ & $<46.0$ & $<44.0$ & & & \\
\hline S95T003072 & & Lower $1 / 2$ & $<78.1$ & $<76.2$ & $<77.2$ & & & \\
\hline S95T003073 & \multirow[t]{2}{*}{$112: 9$} & Upper $1 / 2$ & $<82.8$ & $<75.9$ & $<79.4$ & & & \\
\hline$\$ 95 T 003074$ & & Lower $1 / 2$ & $<68.5$ & $<76.2$ & $<72.4$ & & & \\
\hline \$95T003075 & \multirow[t]{2}{*}{ 112: 10} & Upper $1 / 2$ & $<74.3$ & $<72.7$ & $<73.5$ & & & \\
\hline S95T003076 & & Lower $1 / 2$ & $<91.2$ & $<82.1$ & $<86.7$ & & & \\
\hline \$95T003077 & \multirow[t]{2}{*}{$112: 11$} & Upper $1 / 2$ & $<81.4$ & $<69.7$. & $<75.6$ & & & \\
\hline S95T003078 & & Lower $1 / 2$ & $<190$ & $<229$ & $<209.5$ & & & \\
\hline S95T003079 & \multirow[t]{2}{*}{$112: 12$} & Upper $1 / 2$ & $<214$ & $<193$ & $<203.5$ & & & \\
\hline S95T003080 & & Lower $1 / 2$ & $<196$ & $<\overline{202}$ & $<199.0$ & & & \\
\hline
\end{tabular}




\begin{tabular}{|c|c|c|c|c|c|c|c|c|}
\hline & & & $8: 28>$ & I.06 > & $S^{\circ} S L>$ & $4 / 1$ IəMOT & \multirow[b]{2}{*}{$6: t 11$} & L6ZE00.LS6S \\
\hline & & & $9.28>$ & $6.26>$ & $z^{\prime} z 8>$ & $z_{1}$ Iadd $n$ & & 96ZE00LS6S \\
\hline & & & $s^{\circ} 0 t>$ & $0.00>$ & 0 It $>$ & $2 / 1$ IOMO & \multirow[b]{2}{*}{$8: t I I$} & S6ZE00LS6S \\
\hline & & & $S . L S>$ & $9.1 L>$ & $t^{\circ} \varepsilon t>$ & $z_{1}$ Iədd $n$ & & t6ZE00LS6S \\
\hline & & & $0.1 t>$ & $8.8 \varepsilon>$ & $\mid \cdot \varepsilon b>$ & $y / 1$ JәMO $\mathrm{T}$ & \multirow[b]{2}{*}{$L: t I I$} & E6ZE00」S6S \\
\hline & & & $2 \cdot s t>$ & $s^{\prime} \varepsilon t>$ & $8.9 t>$ & $2 / 1 \operatorname{sadd} \Omega$ & & Z6ZE00 LS6S \\
\hline & & & $6.9 L>$ & $0.58>$ & $L 89>$ & $z_{1}$ دəMO & \multirow[b]{2}{*}{$9:$ tI } & $162 \varepsilon 00 \mathrm{LS} 6 \mathrm{~S}$ \\
\hline & & & $t z t>$ & $9.6 \varepsilon>$ & $\tau \cdot s t>$ & $\not / 12 \mathrm{dd} \cap$ & & 06ZE00 IS6S \\
\hline & & & $0.9 b>$ & $89 t>$ & I. $s t>$ & $\psi_{1 / 1}$ дәмо & \multirow[b]{2}{*}{$s: t I I$} & $68 Z \varepsilon 00 \mathrm{~L} S 6 \mathrm{~S}$ \\
\hline & & & $9.16>$ & $\angle 8 \varepsilon>$ & $0.00>$ & $z / 1$ Iadd $\Omega$ & & $88 z \varepsilon 00 \mathrm{LS} 6 \mathrm{~S}$ \\
\hline & & & $76 L>$ & $588>$ & $2 \cdot 0 L>$ & $y_{1}$ 12M07 & \multirow[b]{2}{*}{$t: t i l$} & $\angle 8 Z \varepsilon 00 \perp S 6 S$ \\
\hline & & & $0.2 t>$ & $6.6 \varepsilon>$ & $s^{*} t t^{\prime}>$ & $y_{1}$ ladd $n$ & & $982 \varepsilon 00 \perp \subseteq 6 \mathrm{~S}$ \\
\hline & & & $29 \varepsilon>$ & $98 \varepsilon>$ & $8 \varepsilon \varepsilon>$ & $y_{1}$ गОМО & \multirow[b]{2}{*}{$\varepsilon: \downarrow l l$} & S8ZE00LS6S \\
\hline & & & $L 8 E>$ & $8 . \angle E>$ & $96 \varepsilon>$ & $z / 1$ Iadd $\Omega$ & & $\$ 8 Z \varepsilon 00 \mathrm{LS} 6 \mathrm{~S}$ \\
\hline & & & $\varepsilon^{\prime} L \varepsilon>$ & $\forall \cdot 9 \varepsilon>$ & $I 8 \mathcal{E}>$ & $y / 12 \mathrm{MO}$ & \multirow[b]{2}{*}{$Z: t I I$} & E8ZE00LSS6S \\
\hline & & & $0^{\circ} \varepsilon>$ & $0.00>$ & $6.11>$ & $z / 1$ Jadd $\Omega$ & & 28ZE00LS6S \\
\hline & & & $0.08 \varepsilon>$ & $29 \varepsilon>$ & $86 \varepsilon>$ & $Z / 12 \partial M O \mathrm{~T}$ & \multirow[b]{2}{*}{ †I:ZI I } & $\$ 80 E 00 \mathrm{LS} 6 \mathrm{~S}$ \\
\hline & & & $\varsigma^{\circ} 861>$ & $z I \tau>$ & S8I $>$ & $\% /$ Iədd $_{n}$ & & E80E00LS6S \\
\hline & & & $s^{\prime} 181>$ & $69 \mathrm{I}>$ & $76 \mathrm{I}>$ & $z_{1}$ IOMO & \multirow[b]{2}{*}{ EI:ZII } & 280E00LS6S \\
\hline & & & $0^{\circ} S L I>$ & $9 L I>$ & $t L I>$ & $z_{1}$ Iadd $\Omega$ & & I80E00.LS6S \\
\hline $8 \%$ & 8. & $6 \%$ & T/W\% & $4 \%$ & $\% \%$ & (1) & 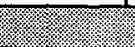 & 90102 \\
\hline 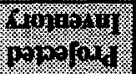 & $\frac{6}{61010}$ & $\frac{1004}{110040}$ & 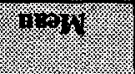 & 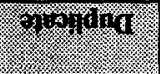 & 10904 & 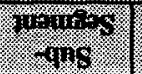 & 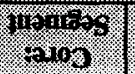 & ofyorigit \\
\hline
\end{tabular}

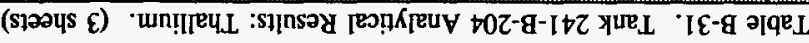


Table B-31. Tank 241-B-204 Analytical Results: Thallium. (3 sheets)

\begin{tabular}{|c|c|c|c|c|c|c|c|c|}
\hline (6) & 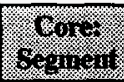 & \% & itenin & 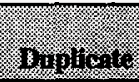 & 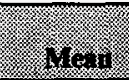 & 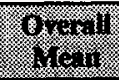 & $\frac{148}{1091 ; 1}$ & 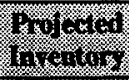 \\
\hline Wous & rে & & $18 \mathrm{c}$ & 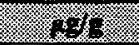 & 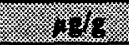 & 等. & , & 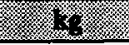 \\
\hline S95T003298 & \multirow[t]{2}{*}{$114: 10$} & Upper $1 / 2$ & $<74.0$ & $<71.7$ & $<72.9$ & & & \\
\hline S95T003299 & & Lower $1 / 2$ & $<67.7$ & $<77.1$ & $<72.4$ & & & \\
\hline$\$ 95 T 003300$ & \multirow[t]{2}{*}{$114: 11$} & Upper $1 / 2$ & $<94.0$ & $<96.6$ & $<95.3$ & & & \\
\hline S95T003301 & & Lower $1 / 2$ & $<68.0$ & $<68.2$ & $<68.1$ & & & \\
\hline S95T003302 & \multirow[t]{2}{*}{$114: 12$} & Upper $1 / 2$ & $<48.0$ & $<44.2$ & $<46.1$ & & & \\
\hline S95T003303 & & Lower $1 / 2$ & $<44.7$ & $<74.1$ & $<59.4$ & & & \\
\hline \$95T003304 & \multirow[t]{2}{*}{$114: 13$} & Upper $1 / 2$ & $<91.6$ & $<81.8$ & $<86.7$ & & & \\
\hline \$95T003305 & & Lower $1 / 2$ & $<72.1$ & $<73.1$ & $<72.6$ & & & \\
\hline$\$ 95 T 003306$ & \multirow[t]{2}{*}{$114: 14$} & Upper $1 / 2$ & $<85.8$ & $<85.5$ & $<85.7$ & & & \\
\hline S95T003307 & & Lower $1 / 2$ & $<77.3$ & $<84.6$ & $<81.0$ & & & \\
\hline \multicolumn{3}{|c|}{ Drathing iquirs } & 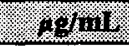 & . & 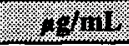 & 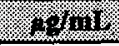 & 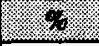 & 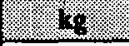 \\
\hline S95T002976 & $112: 1$ & $\mathrm{DL}$ & $<40.2$ & $<40.2$ & $<40.2$ & \multirow[t]{3}{*}{$<30.2$} & \multirow[t]{3}{*}{$n / a$} & \multirow[t]{3}{*}{$<0.121$} \\
\hline S95T002984 & $112: 2$ & $\mathrm{DL}$ & $<40.2$ & $<40.2$ & $<40.2$ & & & \\
\hline S95T003255 & $114: 1$ & DL & $<20.2$ & $<20.2$ & $<20.2$ & & & \\
\hline
\end{tabular}


Table B-32. Tank 241-B-204 Analytical Results: Titanium. (3 sheets)

\begin{tabular}{|c|c|c|c|c|c|c|c|c|}
\hline Singer & 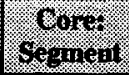 & 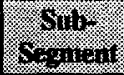 & jowing & Binglon & $\frac{1}{6}$ & 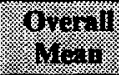 & 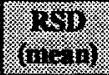 & 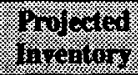 \\
\hline 50110.62 & & & 286 & 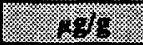 & 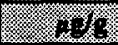 & 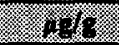 & (2. & (4) \\
\hline S95T002980 & $112: 2$ & Whole & 37.10 & 28.50 & 32.80 & \multirow[t]{21}{*}{$<6.98$} & \multirow[t]{21}{*}{$n / a$} & \multirow[t]{21}{*}{$<1.54$} \\
\hline S95T003061 & \multirow[t]{2}{*}{$112: 3$} & Upper $1 / 2$ & 3.570 & 4.140 & 3.855 & & & \\
\hline S95T003062 & & Lower $1 / 2$ & 2.450 & 2.430 & 2.44 & & & \\
\hline S95T003063 & \multirow[t]{2}{*}{$112: 4$} & Upper $1 / 2$ & 2.200 & 2.860 & 2.530 & & & \\
\hline S95T003064 & & Lower $1 / 2$ & 2.460 & 2.530 & 2.495 & & & \\
\hline S95T003065 & \multirow[t]{2}{*}{$112: 5$} & Upper $1 / 2$ & $<4.37$ & $<4.16$ & $<4.27$ & & & \\
\hline S95T003066 & & Lower $1 / 2$ & 3.900 & 3.620 & 3.760 & & & \\
\hline S95T003067 & \multirow[t]{2}{*}{$112: 6$} & Upper $1 / 2$ & $<4.66$ & 4.350 & 4.505 & & & \\
\hline \$95T003068 & & Lower $1 / 2$ & 4.580 & 3.930 & 4.255 & & & \\
\hline S95T003069 & \multirow[t]{2}{*}{$112: 7$} & Upper $1 / 2$ & 4.060 & 4.710 & 4.385 & & & \\
\hline S95T003070 & & Lower $1 / 2$ & 4.180 & 3.990 & 4.085 & & & \\
\hline S95T003071 & \multirow[t]{2}{*}{$112: 8$} & Upper $1 / 2$ & 3.060 & 3.290 & 3.175 & & & \\
\hline S95T003072 & & Lower $1 / 2$ & $<3.91$ & 4.710 & 4.31 & & & \\
\hline S95T003073 & \multirow[t]{2}{*}{$112: 9$} & Upper $1 / 2$ & 4.300 & 5.710 & 5.005 & & & \\
\hline S95T003074 & & Lower $1 / 2$ & $<3.43$ & $<3.81$ & $<3.62$ & & & \\
\hline S95T003075 & \multirow[t]{2}{*}{$112: 10$} & Upper $1 / 2$ & $<3.72$ & $<3.64$ & $<3.68$ & & & \\
\hline S95T003076 & & Lower $1 / 2$ & $<4.56$ & $<4.11$ & $<4.34$ & & & \\
\hline S95T003077 & \multirow[t]{2}{*}{ 112:11 } & Upper $1 / 2$ & $<4.07$ & $<3.48$ & $<3.78$ & & & \\
\hline S95T003078 & & Lower $1 / 2$ & $<9.49$ & $<11.5$ & $<10.5$ & & & \\
\hline S95T003079 & \multirow[t]{2}{*}{$112: 12$} & Upper $1 / 2$ & $<10.7$ & $<9.66$ & $<10.2$ & & & \\
\hline S95T003080 & & Lower $1 / 2$ & $<9.78$ & $<10.1$ & $<9.94$ & & & \\
\hline
\end{tabular}


Table B-32. Tank 241-B-204 Analytical Results: Titanium. (3 sheets)

\begin{tabular}{|c|c|c|c|c|c|c|c|c|}
\hline Oarmo & 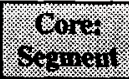 & 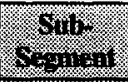 & Blo-ini & B. & $4=$ & (5) & $\begin{array}{l}260 \\
18 \%=11\end{array}$ & 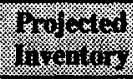 \\
\hline 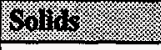 & (1) & (1) & (1) & . 35 & (1) & 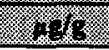 & 3.13 & (1) \\
\hline S95T003081 & \multirow[t]{2}{*}{$112: 13$} & Upper $1 / 2$ & $<8.72$ & $<8.73$ & $<8.725$ & & & \\
\hline S95T003082 & & Lower $1 / 2$ & $<9.72$ & $<8.47$ & $<9.095$ & & & \\
\hline S95T003083 & \multirow[t]{2}{*}{ 112:14 } & Upper $1 / 2$ & $<9.26$ & $<10.6$ & $<57.63$ & & & \\
\hline S95T003084 & & Lower $1 / 2$ & $<19.9$ & $<18.1$ & $<19.0$ & & & \\
\hline S95T003282 & \multirow[t]{2}{*}{$114: 2$} & Upper $1 / 2$ & 3.280 & 3.250 & 3.265 & & & \\
\hline S95T003283 & & Lower $1 / 2$ & $<1.95$ & $<1.82$ & $<1.885$ & & & \\
\hline S95T003284 & \multirow[t]{2}{*}{$114: 3$} & Upper $1 / 2$ & 2.010 & 1.930 & 1.970 & & & \\
\hline S95T003285 & & Lower $1 / 2$ & $<16.9$ & $<19.3$ & $<18.10$ & & & \\
\hline S95T003286 & \multirow[t]{2}{*}{$114: 4$} & Upper $1 / 2$ & $<2.23$ & $<2.040$ & $<2.135$ & & & \\
\hline S95T003287 & & Lower $1 / 2$ & $<3.51$ & $<4.43$ & $<3.970$ & & & \\
\hline S95T003288 & \multirow[t]{2}{*}{$114: 5$} & Upper $1 / 2$ & 3.260 & 2.980 & 3.120 & & & \\
\hline S95T003289 & & Lower $1 / 2$ & 4.050 & 3.780 & 3.915 & & & \\
\hline S95T003290 & \multirow[t]{2}{*}{$114: 6$} & Upper $1 / 2$ & 3.930 & 3.790 & 3.860 & & & \\
\hline S95T003291 & & Lower $1 / 2$ & 3.760 & $<4.25$ & 4.005 & & & \\
\hline S95T003292 & \multirow[t]{2}{*}{$114: 7$} & Upper $1 / 2$ & 3.860 & 3.960 & 3.910 & & & \\
\hline S95T003293 & & Lower $1 / 2$ & 3.290 & 3.110 & 3.200 & & & \\
\hline S95T003294 & \multirow[t]{2}{*}{$114: 8$} & Upper $1 / 2$ & 4.680 & 4.130 & 4.405 & & & \\
\hline S95T003295 & & Lower $1 / 2$ & 3.080 & 3.000 & 3.040 & & & \\
\hline S95T003296 & \multirow[t]{2}{*}{$114: 9$} & Upper $1 / 2$ & $<4.11$ & $<4.64$ & $<4.375$ & & & \\
\hline S95T003297 & & Lower $1 / 2$ & $<3.79$ & $<4.50$ & $<4.145$ & & & \\
\hline
\end{tabular}


Table B-32. Tank 241-B-204 Analytical Results: Titanium. (3 sheets)

\begin{tabular}{|c|c|c|c|c|c|c|c|c|}
\hline 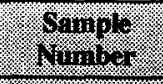 & 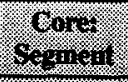 & $\frac{10}{4019}$ & Iesin! & Mopiciole & 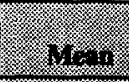 & $\frac{6}{6}$ & 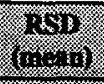 & 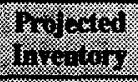 \\
\hline Solos & & (1) & 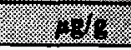 & (1) & 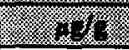 & r. & . & s. \\
\hline S95T003298 & \multirow[t]{2}{*}{ 114: 10} & Upper $1 / 2$ & $<3.70$ & $<3.59$ & $<3.645$ & & & \\
\hline S95T003299 & & Lower $1 / 2$ & $<3.39$ & $<3.85$ & $<3.620$ & & & \\
\hline \$95T003300 & \multirow[t]{2}{*}{$114: 11$} & Upper $1 / 2$ & $<4.70$ & $<4.83$ & $<4.765$ & & & \\
\hline S95T003301 & & Lower $1 / 2$ & 3.610 & 3.460 & 3.535 & & & \\
\hline S95T003302 & \multirow[t]{2}{*}{$114: 12$} & Upper $1 / 2$ & 2.840 & 2.890 & 2.865 & & & \\
\hline S95T003303 & & Lower $1 / 2$ & 3.360 & $<3.70$ & 3.53 & & & \\
\hline S95T003304 & \multirow[t]{2}{*}{$114: 13$} & Upper $1 / 2$ & $<4.58$ & 4.140 & 4.36 & & & \\
\hline S95T003305 & & Lower $1 / 2$ & $<3.60$ & $<3.66$ & $<3.630$ & & & \\
\hline S95T003306 & \multirow[t]{2}{*}{ 114:14 } & Upper $1 / 2$ & $<4.29$ & $<4.27$ & $<4.280$ & & & \\
\hline$\$ 95 T 003307$ & & Lower $1 / 2$ & $<3.86$ & $<4.22$ & $<4.040$ & & & \\
\hline \multicolumn{3}{|c|}{ Drainable ngulds } & (1) & 25101 & (2) & $1 / 21118$ & 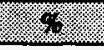 & 䇥, \\
\hline \$95T002976 & 112:1 & $\mathrm{DL}$ & $<2.01$ & $<2.01$ & $<2.01$ & \multirow[t]{3}{*}{$<1.51$} & \multirow[t]{3}{*}{$n / a$} & \multirow[t]{3}{*}{$<0.00604$} \\
\hline S95T002984 & $112: 2$ & DL & $<2.01$ & $<2.01$ & $<2.01$ & & & \\
\hline S95T003255 & $114: 1$ & $\mathrm{DL}$ & $<1.01$ & $<1.01$ & $<1.01$ & & & \\
\hline
\end{tabular}


Table B-33. Tank 241-B-204 Analytical Results: Uranium. (3 sheets)

\begin{tabular}{|c|c|c|c|c|c|c|c|c|}
\hline winger & $x^{6} x^{6}$ & (x) & Pinisin & $(8,3,1)=4$ & (3) & 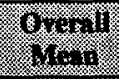 & (10) & 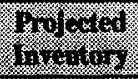 \\
\hline Soligs. & 4 & & 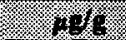 & 47 & 35 & 40 & . & 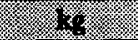 \\
\hline \$95T002980 & $112: 2$ & Whole & 373.0 & 351.0 & 362.0 & \multirow[t]{21}{*}{$<240$} & \multirow[t]{21}{*}{$\mathrm{n} / \mathrm{a}$} & \multirow[t]{21}{*}{$<52.8$} \\
\hline S95T003061 & \multirow[t]{2}{*}{$112: 3$} & Upper $1 / 2$ & $<120$ & $<94.2$ & $<107$ & & & \\
\hline S95T003062 & & Lower $1 / 2$ & $<112$ & $<106$ & $<109$ & & & \\
\hline \$95T003063 & \multirow[t]{2}{*}{$112: 4$} & Upper $1 / 2$ & $<87.8$ & $<116$ & $<101.9$ & & & \\
\hline S95T003064 & & Lower $1 / 2$ & $<\overline{121}$ & $<111$ & $<116$ & & & \\
\hline S95T003065 & \multirow[t]{2}{*}{$112: 5$} & Upper $1 / 2$ & $<218$ & $<208$ & $<213$ & & & \\
\hline S95T003066 & & Lower $1 / 2$ & $<115$ & $<97.7$ & $<106.4$ & & & \\
\hline S95T003067 & \multirow[t]{2}{*}{$112: 6$} & Upper $1 / 2$ & $<233$ & $<172$ & $<202.5$ & & & \\
\hline S95T003068 & & Lower $1 / 2$ & $<111$ & $<96.9$ & $<104.0$ & & & \\
\hline \$95T003069 & \multirow[t]{2}{*}{$112: 7$} & Upper $1 / 2$ & $<182$ & $<190$ & $<186$ & & & \\
\hline S95T003070 & & Lower $1 / 2$ & $<176$ & $<171$ & $<173.5$ & & & \\
\hline S95T003071 & \multirow[t]{2}{*}{$112: 8$} & Upper $1 / 2$ & $<105$ & $<115$ & $<110$ & & & \\
\hline S95T003072 & & Lower $1 / 2$ & $<195$ & $<191$ & $<193$ & & & \\
\hline S95T003073 & \multirow[t]{2}{*}{$112: 9$} & Upper $1 / 2$ & $<207$ & $<190$ & $<198.5$ & & & \\
\hline S95T003074 & & Lower $1 / 2$ & $<171$ & $<191$ & $<181$ & & & \\
\hline S95T003075 & \multirow[t]{2}{*}{$112: 10$} & Upper $1 / 2$ & $<186$ & $<.182$ & $<184$ & & & \\
\hline S95T003076 & & Lower $1 / 2$ & $<228$ & $<205$ & $<216.5$ & & & \\
\hline S95T003077 & \multirow[t]{2}{*}{$112: 11$} & Upper $1 / 2$ & $<203$ & $<174$ & $<188.5$ & & & \\
\hline S95T003078 & & Lower $1 / 2$ & $<474$ & $<574$ & $<524$ & & & \\
\hline S95T003079 & \multirow[t]{2}{*}{$112: 12$} & Upper $1 / 2$ & $<535$ & $<483$ & $<509$ & & & \\
\hline S95T003080 & & Lower $1 / 2$ & $<489$ & $<504$ & $<496.5$ & & & \\
\hline
\end{tabular}


Table B-33. Tank 241-B-204 Analytical Results: Uranium. (3 sheets)

\begin{tabular}{|c|c|c|c|c|c|c|c|c|}
\hline 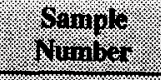 & ofosit) & 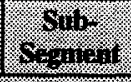 & prill & 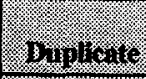 & 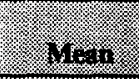 & $8 \%$ & $\frac{3013}{(18,11)}$ & 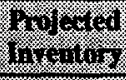 \\
\hline Goims & 4 & 2. & 16to & 28 & 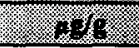 & 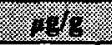 & \% & 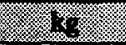 \\
\hline S95T003081 & \multirow[t]{2}{*}{$112: 13$} & Upper $1 / 2$ & $<436$ & $<436$ & $<436$ & & & \\
\hline S95T003082 & & Lower $1 / 2$ & $<486$ & $<424$ & $<455$ & & & \\
\hline S95T003083 & \multirow[t]{2}{*}{$112: 14$} & Upper $1 / 2$ & $<463$ & $<529$ & $<496$ & & & \\
\hline S95T003084 & & Lower $1 / 2$ & $<996$ & $<905$ & $<950.5$ & & & \\
\hline S95T003282 & \multirow[t]{2}{*}{$114: 2$} & Upper $1 / 2$ & 306.0 & 298.0 & 302.0 & & & \\
\hline \$95T003283 & & Lower $1 / 2$ & 300.0 & 322.0 & 311.0 & & & \\
\hline S95T003284 & \multirow[t]{2}{*}{$114: 3$} & Upper $1 / 2$ & $<99.0$ & $<94.4$ & $<96.7$ & & & \\
\hline \$95T003285 & & Lower $1 / 2$ & $<845$ & $<966$ & $<905.5$ & & & \\
\hline S95T003286 & \multirow[t]{2}{*}{$114: 4$} & Upper $1 / 2$ & $<111$ & $<98.4$ & $<104.7$ & & & \\
\hline S95T003287 & & Lower $1 / 2$ & $<177$ & $<221$ & $<199$ & & & \\
\hline \$95T003288 & \multirow[t]{2}{*}{$114: 5$} & Upper $1 / 2$ & $<111$ & $<96.6$ & $<103.8$ & & & \\
\hline S95T003289 & & Lower $1 / 2$ & $<113$ & $<117$ & $<115$ & & & \\
\hline$\$ 95 T 003290$ & \multirow[t]{2}{*}{$114: 6$} & Upper $1 / 2$ & $<113$ & $<99.0$ & $<106$ & & & \\
\hline \$95T003291 & & Lower $1 / 2$ & $<172$ & $<213$ & $<192.5$ & & & \\
\hline S95T003292 & \multirow[t]{2}{*}{$114: 7$} & Upper $1 / 2$ & $<117$ & $<109$ & $<113$ & & & \\
\hline S95T003293 & & Lower $1 / 2$ & $<108$ & $<96.9$ & $<102.5$ & & & \\
\hline S95T003294 & \multirow[t]{2}{*}{$114: 8$} & Upper $1 / 2$ & $<110$ & $<179$ & $<144.5$ & & & \\
\hline S95T003295 & & Lower $1 / 2$ & $<104$ & $<100$ & $<102$ & & & \\
\hline \$95T003296 & \multirow[t]{2}{*}{$114: 9$} & Upper $1 / 2$ & $<207$ & $<232$ & $<219.5$ & & & \\
\hline S95T003297 & & Lower $1 / 2$ & $<189$ & $<225$ & $<207$ & & & \\
\hline
\end{tabular}


Table B-33. Tank 241-B-204 Analytical Results: Uranium. (3 sheets)

\begin{tabular}{|c|c|c|c|c|c|c|c|c|}
\hline Mons & 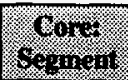 & 20 & nowin & $(3,4)=0$ & 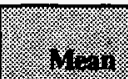 & 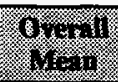 & $\begin{array}{c}343 \\
(3,1)\end{array}$ & 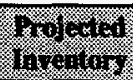 \\
\hline Sol16s & $x^{\prime}$ & 湾 & 816 & 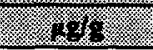 & 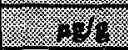 & 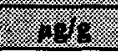 & (1) & সে \\
\hline \$95T003298 & \multirow[t]{2}{*}{$114: 10$} & Upper $1 / 2$ & $<185$ & $<179$ & $<182$ & & & \\
\hline \$95T003299 & & Lower $1 / 2$ & $<169$ & $<193$ & $<177.5$ & & & \\
\hline S95T003300 & \multirow[t]{2}{*}{$114: 11$} & Upper $1 / 2$ & $<235$ & $<241$ & $<238$ & & & \\
\hline S95T003301 & & Lower $1 / 2$ & $<170$ & $<170$ & $<170$ & & & \\
\hline S95T003302 & \multirow[t]{2}{*}{$114: 12$} & Upper $1 / 2$ & $<120$ & $<110$ & $<115$ & & & \\
\hline S95T003303 & & Lower $1 / 2$ & $<112$ & $<185$ & $<148.5$ & & & \\
\hline S95T003304 & \multirow[t]{2}{*}{$114: 13$} & Upper $1 / 2$ & $<229$ & $<204$ & $<216.5$ & & & \\
\hline S95T003305 & & Lower $1 / 2$ & $<180$ & $<183$ & $<181.5$ & & & \\
\hline S95T003306 & \multirow[t]{2}{*}{$114: 14$} & Upper $1 / 2$ & $<216$ & $<214$ & $<215$ & & & \\
\hline S95T003307 & & Lower $1 / 2$ & $<193$ & $<211$ & $<202$ & & & \\
\hline \multicolumn{3}{|c|}{ Brumble rigros } & ofin & $8 / 111$. & 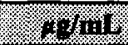 & $(2)$ init & (5. & 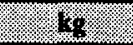 \\
\hline S95T002976 & 112:1 & DL & 179.0 & 195.0 & 187.0 & \multirow[t]{3}{*}{164} & \multirow[t]{3}{*}{7.9} & \multirow[t]{3}{*}{0.656} \\
\hline S95T002984 & $112: 2$ & $\mathrm{DL}$ & 173.0 & 163.0 & 168.0 & & & \\
\hline S95T003255 & $114: 1$ & $\mathrm{DL}$ & 150.0 & 152.0 & 151.0 & & & \\
\hline
\end{tabular}




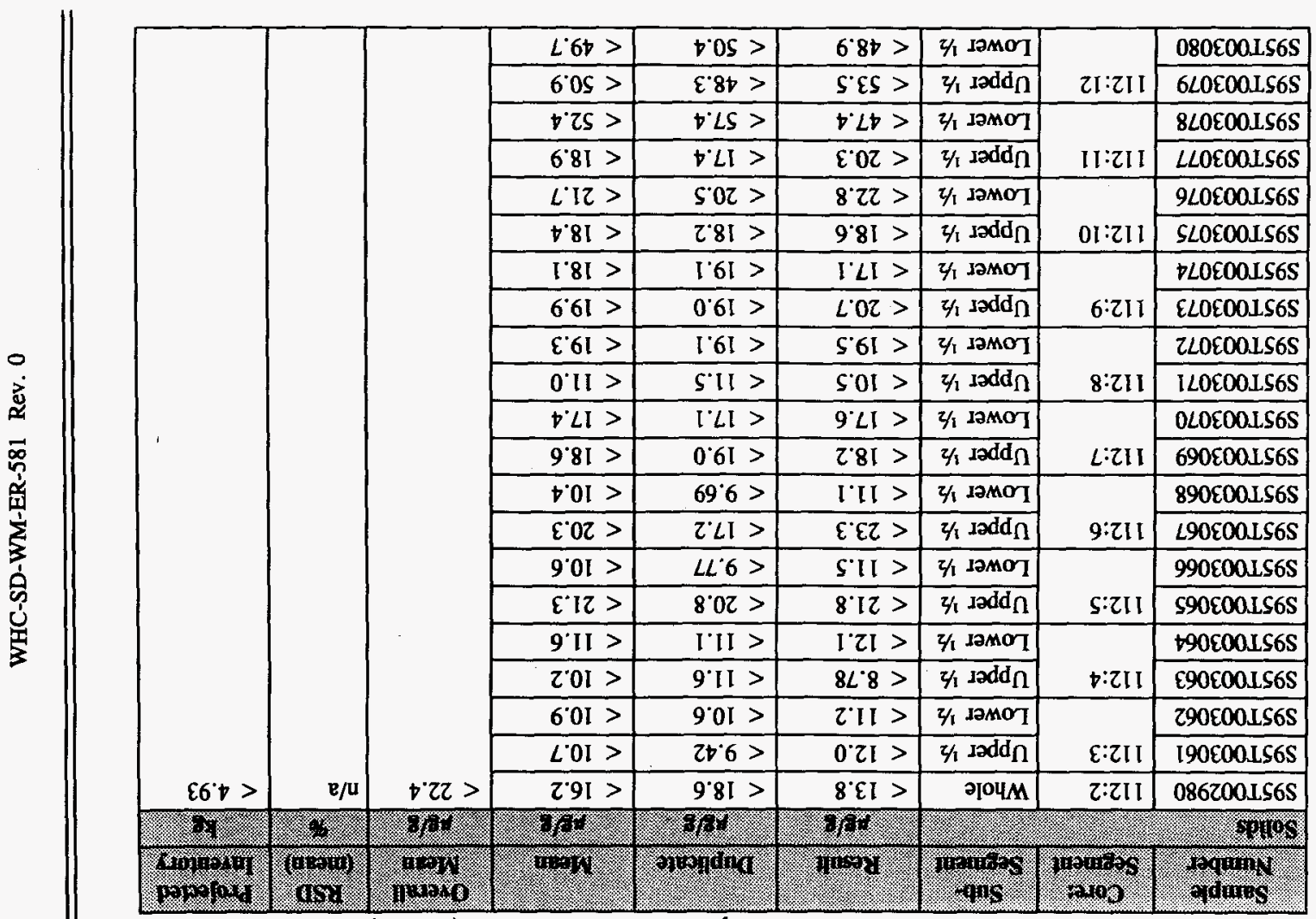


WHC-SD-WM-ER-581 Rev. 0

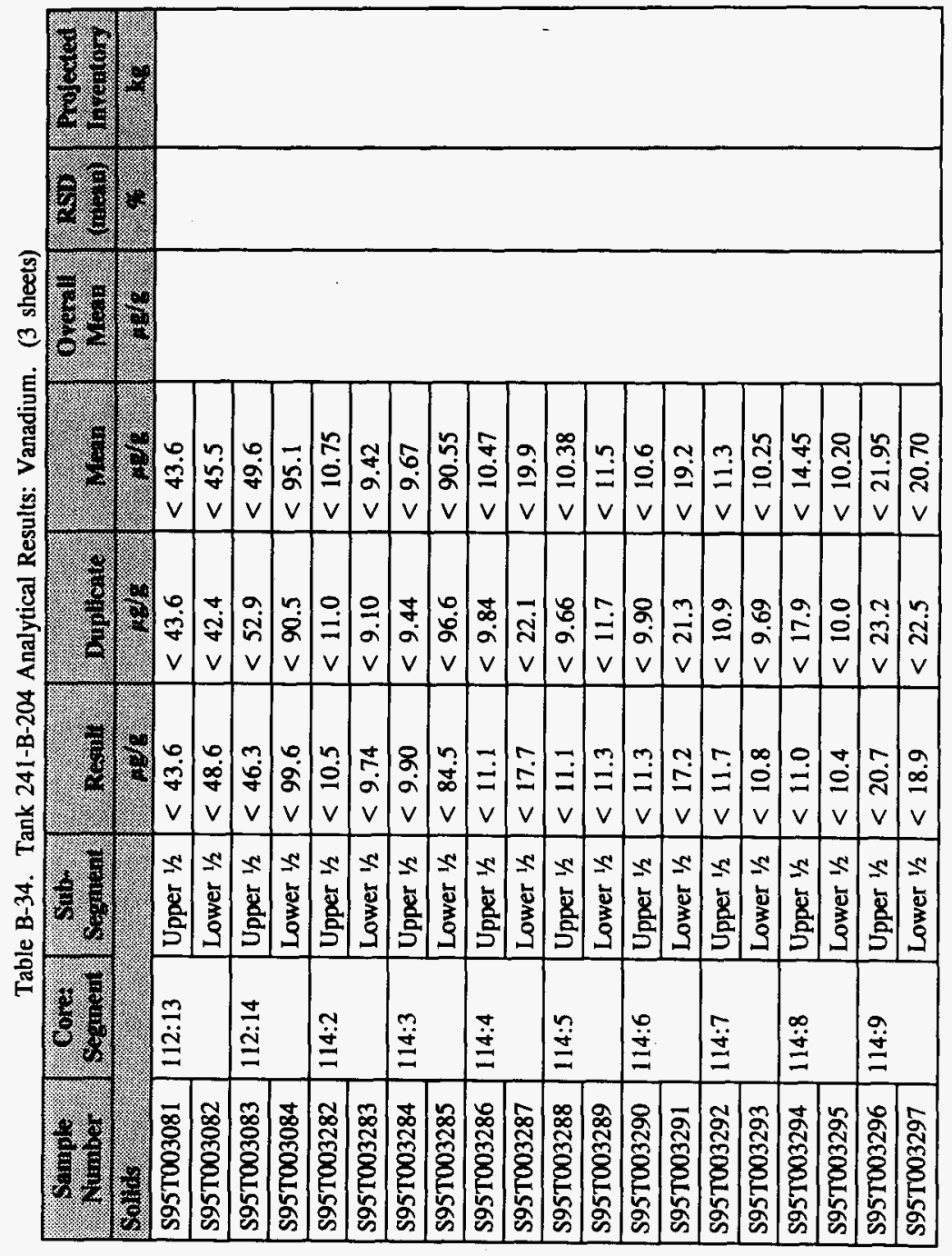


WHC-SD-WM-ER-581 Rev. 0

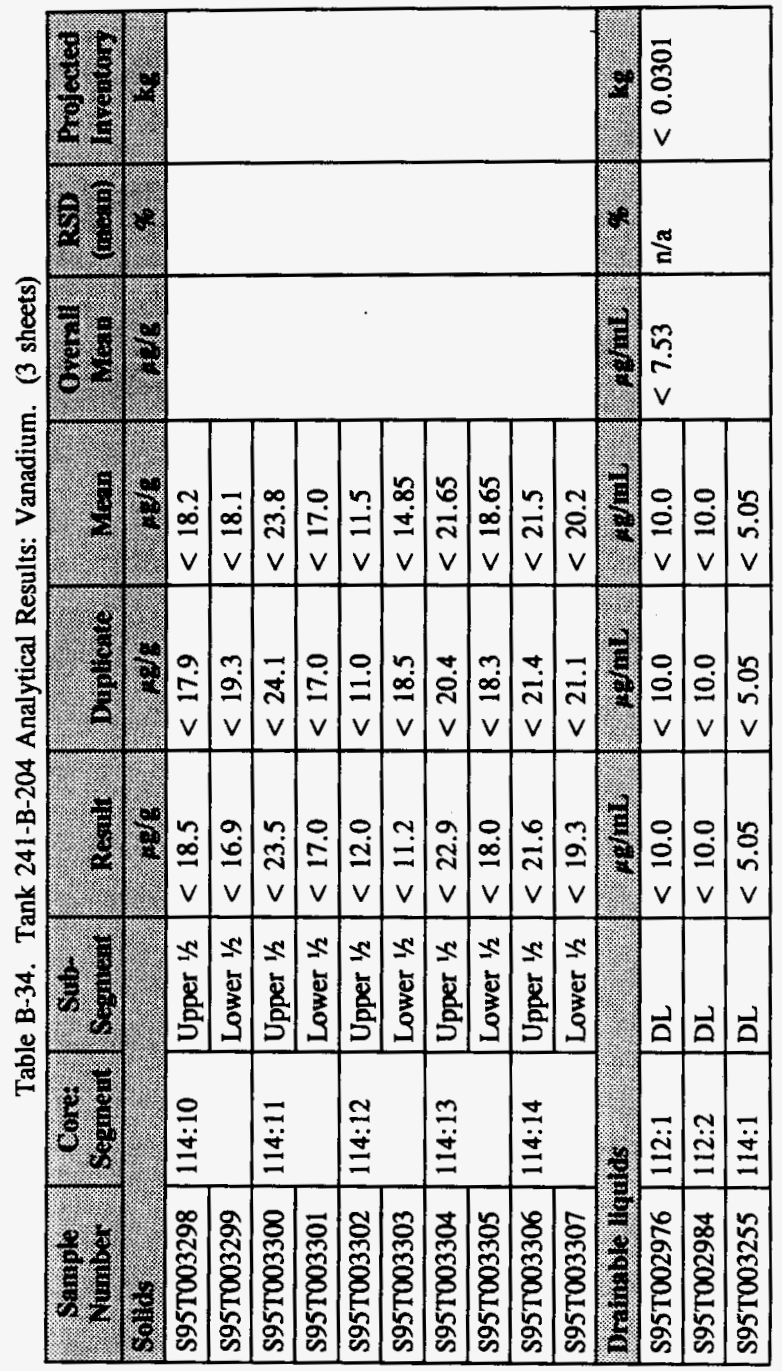


Table B-35. Tank 241-B-204 Analytical Results: Zinc. (4 sheets)

\begin{tabular}{|c|c|c|c|c|c|c|c|c|}
\hline 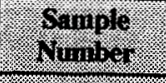 & orot & 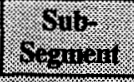 & 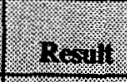 & Denl) & M.1. & 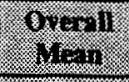 & 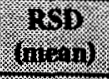 & 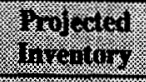 \\
\hline 6015 & & 4 & 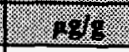 & 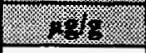 & 2.218 & 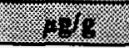 & r. & 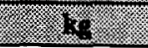 \\
\hline S95T002980 & $112: 2$ & Whole & 178.0 & 149.0 & 163.5 & \multirow[t]{21}{*}{53.7} & \multirow[t]{21}{*}{39.7} & \multirow[t]{21}{*}{11.8} \\
\hline $595 T 003061$ & \multirow[t]{2}{*}{$112: 3$} & Upper $1 / 2$ & 19.80 & 16.90 & 18.35 & & & \\
\hline \$95T003062 & & Lower $1 / 2$ & 11.50 & 11.20 & 11.35 & & & \\
\hline S95T003063 & \multirow[t]{2}{*}{$112: 4$} & Upper $1 / 2$ & 20.90 & 27.80 & 24.35 & & & \\
\hline S95T003064 & & Lower $1 / 2$ & 20.50 & 12.40 & 16.45 & & & \\
\hline S95T003065 & \multirow[t]{2}{*}{$112: 5$} & Upper $1 / 2$ & 15.30 & 10.20 & 12.75 & & & \\
\hline S95T003066 & & Lower $1 / 2$ & 14.40 & 8.78 & 11.59 & & & \\
\hline S95T003067 & \multirow[t]{2}{*}{$112: 6$} & Upper $1 / 2$ & 12.10 & 22.40 & 17.25 & & & \\
\hline S95T003068 & & Lower $1 / 2$ & 14.70 & 13.10 & 13.90 & & & \\
\hline S95T003069 & \multirow[t]{2}{*}{$112: 7$} & Upper $1 / 2$ & 11.20 & 15.30 & 13.25 & & & \\
\hline S95T003070 & & Lower $1 / 2$ & 7.96 & 7.92 & 7.94 & & & \\
\hline S95T003071 & \multirow[t]{2}{*}{$112: 8$} & Upper $1 / 2$ & $6 . \overline{48}$ & 9.72 & 8.10 & & & \\
\hline S95T003072 & & Lower $1 / 2$ & 20.40 & 12.10 & 16.25 & & & \\
\hline \$95T003073 & \multirow[t]{2}{*}{$112: 9$} & Upper $1 / 2$ & 15.90 & 19.30 & 17.6 & & & \\
\hline S95T003074 & & Lower $1 / 2$ & 14.90 & 11.10 & 13.00 & & & \\
\hline S95T003075 & \multirow[t]{2}{*}{$112: 10$} & Upper $1 / 2$ & 17.70 & 19.60 & 18.65 & & & \\
\hline S95T003076 & & Lower $1 / 2$ & 17.40 & 27.70 & 22.55 & & & \\
\hline S95T003077 & \multirow[t]{2}{*}{$112: 11$} & Upper $1 / 2$ & 31.30 & 29.30 & 30.30 & & & \\
\hline S95T003078 & & Lower $1 / 2$ & 17.10 & 18.20 & 17.65 & & & \\
\hline S95T003079 & \multirow[t]{2}{*}{$112: 12$} & Upper $1 / 2$ & 12.30 & 18.40 & 15.35 & & & \\
\hline \$95T003080 & & Lower $1 / 2$ & 12.20 & $<10.1$ & 11.15 & & & \\
\hline
\end{tabular}


Table B-35. Tank 241-B-204 Analytical Results: Zinc. (4 sheets)

\begin{tabular}{|c|c|c|c|c|c|c|c|c|}
\hline O., & 60 & 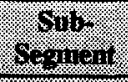 & Ite-ini) & $0,1,11011 \%$ & Men: & 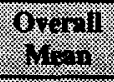 & 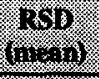 & 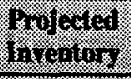 \\
\hline \%olis & & & 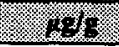 & 18/ & 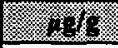 & 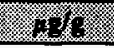 & 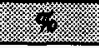 & 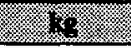 \\
\hline S95T003081 & \multirow[t]{2}{*}{$112: 13$} & Upper $1 / 2$ & 22.80 & 25.40 & 24.10 & & & \\
\hline S95T003082 & & Lower $1 / 2$ & 94.20 & 88.20 & 91.20 & & & \\
\hline S95T003083 & \multirow[t]{2}{*}{$112: 14$} & Upper $1 / 2$ & 338.0 & 274.0 & 306.0 & & & \\
\hline S95T003084 & & Lower $1 / 2$ & 410.0 & 373.0 & 391.5 & & & \\
\hline S95T003282 & \multirow[t]{2}{*}{$114: 2$} & Upper $1 / 2$ & 11.40 & 11.80 & 11.60 & & & \\
\hline S95T003283 & & Lower $1 / 2$ & 7.430 & 8.820 & 8.125 & & & \\
\hline S95T003284 & \multirow[t]{2}{*}{$114: 3$} & Upper $1 / 2$ & 6.410 & 6.290 & 6.350 & & & \\
\hline S95T003285 & & Lower $1 / 2$ & $<16.9$ & $<19.3$ & $<18.1$ & & & \\
\hline \$95T003286 & \multirow[t]{2}{*}{$114: 4$} & Upper $1 / 2$ & 7.190 & 8.740 & 7.965 & & & \\
\hline \$95T003287 & & Lower $1 / 2$ & 8.560 & 8.670 & 8.615 & & & \\
\hline S95T003288 & \multirow[t]{2}{*}{$114: 5$} & Upper $1 / 2$ & 18.50 & 8.690 & 13.59 & & & \\
\hline S95T003289 & & Lower $1 / 2$ & 6.740 & 22.30 & 14.52 & & & \\
\hline S95T003290 & \multirow[t]{2}{*}{$114: 6$} & Upper $1 / 2$ & 12.40 & 11.60 & 12.00 & & & \\
\hline S95T003291 & & Lower $1 / 2$ & 11.20 & 8.810 & 10.00 & & & \\
\hline \$95T003292 & \multirow[t]{2}{*}{$114: 7$} & Upper $1 / 2$ & 7.060 & 7.310 & 7.185 & & & \\
\hline \$95T003293 & & Lower $1 / 2$ & 13.50 & 9.850 & 11.68 & & & \\
\hline S95T003294 & \multirow[t]{2}{*}{$114: 8$} & Upper $1 / 2$ & 96.50 & 59.00 & 77.75 & & & \\
\hline S95T003295 & & Lower $1 / 2$ & 7.860 & 11.50 & 9.680 & & & \\
\hline S95T003296 & \multirow[t]{2}{*}{$114: 9$} & Upper $1 / 2$ & 14.40 & 12.10 & 13.25 & & & \\
\hline S95T003297 & & Lower $1 / 2$ & 19.40 & 15.70 & 17.55 & & & \\
\hline
\end{tabular}




\begin{tabular}{|c|c|c|c|c|c|c|c|c|}
\hline Miniog & 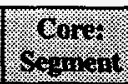 & 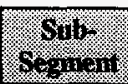 & Inouin & 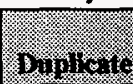 & $f^{2}$ & 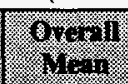 & $\frac{103}{14}$ & 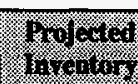 \\
\hline \multicolumn{3}{|l|}{50116} & 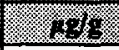 & 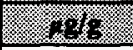 & . & . 31 . & . & r. \\
\hline S95T003298 & \multirow[t]{2}{*}{$114: 10$} & Upper $1 / 2$ & 23.00 & 17.80 & 20.40 & & & \\
\hline S95T003299 & & Lower $1 / 2$ & 14.60 & 12.00 & 13.30 & & & \\
\hline S95T003300 & \multirow[t]{2}{*}{$114: 11$} & Upper $1 / 2$ & 50.80 & 11.50 & 31.15 & & & \\
\hline S95T003301 & & Lower $1 / 2$ & 13.00 & 10.60 & 11.80 & & & \\
\hline S95T003302 & \multirow[t]{2}{*}{$114: 12$} & Upper $1 / 2$ & 10.00 & 16.80 & 13.40 & & & \\
\hline S95T003303 & & Lower $1 / 2$ & 11.40 & 8.100 & 9.750 & & & \\
\hline S95T003304 & \multirow[t]{2}{*}{$114: 13$} & Upper $1 / 2$ & 15.20 & 15.20 & 15.20 & & & \\
\hline S95T003305 & & Lower $1 / 2$ & 65.40 & 65.40 & 65.40 & & & \\
\hline S95T003306 & \multirow[t]{2}{*}{$114: 14$} & Upper $1 / 2$ & 262.0 & 260.0 & 261.0 & & & \\
\hline S95T003307 & & Lower $1 / 2$ & 626.0 & 662.0 & 644.0 & & & \\
\hline \multicolumn{3}{|c|}{ Brinible ligulds. } & 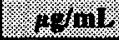 & $28+111$ & (2, in & $26 / 114$ & . & $1 \%$ \\
\hline S95T002976 & $112: 1$ & DL & 8.84 & $<2.01$ & 5.42 & \multirow[t]{3}{*}{2.62} & \multirow[t]{3}{*}{41.2} & \multirow[t]{3}{*}{0.0105} \\
\hline S95T002984 & $112: 2$ & $\mathrm{DL}$ & $<2.01$ & $<2.01$ & $<2.01$ & & & \\
\hline S95T003255 & $114: 1$ & DL & 1.560 & 1.470 & 1.515 & & & \\
\hline
\end{tabular}


Table B-36. Tank 241-B-204 Analytical Results: Zirconium. (3 sheets)

\begin{tabular}{|c|c|c|c|c|c|c|c|c|}
\hline Ginple & 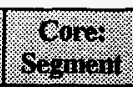 & 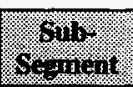 & nesing & oulisicis & . & 6 Mrem & 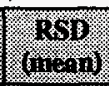 & 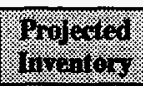 \\
\hline 180110.3. & & & 28 & 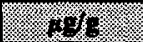 & 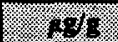 & s. & . & (4) \\
\hline S95T002980 & $112: 2$ & Whole & 4.040 & 4.090 & 4.065 & \multirow[t]{21}{*}{$<4.52$} & \multirow[t]{21}{*}{$n / a$} & \multirow[t]{21}{*}{$<0.995$} \\
\hline S95T003061 & \multirow[t]{2}{*}{$112: 3$} & Upper $1 / 2$ & $<2.41$ & $<1.88$ & $<2.15$ & & & \\
\hline S95T003062 & & Lower $1 / 2$ & $<2.25$ & $<2.13$ & $<2.19$ & & & \\
\hline S95T003063 & \multirow[t]{2}{*}{$112: 4$} & Upper $1 / 2$ & $<1.76$ & $<2.33$ & $<2.05$ & & & \\
\hline S95T003064 & & Lower $1 / 2$ & $<2.43$ & $<2.22$ & $<2.33$ & & & \\
\hline S95T003065 & \multirow[t]{2}{*}{$112: 5$} & Upper $1 / 2$ & $<4.37$ & $<4.16$ & $<4.27$ & & & \\
\hline \$95T003066 & & Lower $1 / 2$ & $<2.31$ & $<2.22$ & $<2.27$ & & & \\
\hline S95T003067 & \multirow[t]{2}{*}{$112: 6$} & Upper $1 / 2$ & $<4.66$ & $<3.46$ & $<4.06$ & & & \\
\hline \$95T003068 & & Lower $1 / 2$ & $<2.23$ & $<1.94$ & $<2.09$ & & & \\
\hline \$95T003069 & \multirow[t]{2}{*}{$112: 7$} & Upper $1 / 2$ & $<3.64$ & $<3.81$ & $<7.45$ & & & \\
\hline S951003070 & & Lower $1 / 2$ & $<3.51$ & $<3.43$ & $<3.47$ & & & \\
\hline S95T003071 & \multirow[t]{2}{*}{$112: 8$} & Upper $1 / 2$ & $<2.10$ & $<2.30$ & $<2.20$ & & & \\
\hline \$95T003072 & & Lower $1 / 2$ & $<3.91$ & $<3.81$ & $<3.86$ & & & \\
\hline \$95T003073 & \multirow[t]{2}{*}{$112: 9$} & Upper $1 / 2$ & $<4.14$ & $<3.80$ & $<3.97$ & & & \\
\hline S95T003074 & & Lower $1 / 2$ & $<3.43$ & $<3.81$ & $<3.62$ & & & \\
\hline S95T003075 & \multirow[t]{2}{*}{$112: 10$} & Upper $1 / 2$ & $<3.72$ & $<3.64$ & $<3.68$ & & & \\
\hline S95T003076 & & Lower $1 / 2$ & $<4.56$ & $<4.11$ & $<4.34$ & & & \\
\hline S95T003077 & \multirow[t]{2}{*}{$112: 11$} & Upper $1 / 2$ & $<4.07$ & $<3.48$ & $<3.78$ & & & \\
\hline S95T003078 & & Lower $1 / 2$ & $<9.49$ & $<11.5$ & $<10.50$ & & & \\
\hline S95T003079 & \multirow[t]{2}{*}{$112: 12$} & Upper $1 / 2$ & $<10.7$ & $<9.66$ & $<10.18$ & & & \\
\hline S95T003080 & & Lower $1 / 2$ & $<9.78$ & $<10.1$ & $<9.94$ & & & \\
\hline
\end{tabular}


Table B-36. Tank 241-B-204 Analytical Results: Zirconium. (3 sheets)

\begin{tabular}{|c|c|c|c|c|c|c|c|c|}
\hline 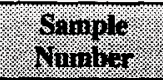 & 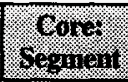 & (2) & (x) & minglisage & 16 eris & $4 \%$ & 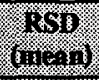 & 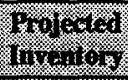 \\
\hline 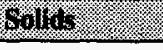 & & & 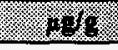 & $8+4$ & $8 \%$ & (1) & (1) & (1) \\
\hline S95T003081 & \multirow[t]{2}{*}{$112: 13$} & Upper $1 / 2$ & $<8.72$ & $<8.73$ & $<8.73$ & & & \\
\hline S95T003082 & & Lower $1 / 2$ & $<9.72$ & $<8.47$ & $<9.10$ & & & \\
\hline S95T003083 & \multirow[t]{2}{*}{$112: 14$} & Upper $1 / 2$ & $<9.26$ & $<10.6$ & $<9.93$ & & & \\
\hline S95T003084 & & Lower $1 / 2$ & $<19.9$ & $<18.1$ & $<19.0$ & & & \\
\hline S95T003282 & \multirow[t]{2}{*}{$114: 2$} & Upper $1 / 2$ & $<2.10$ & $<2.20$ & $<2.15$ & & & \\
\hline S95T003283 & & Lower $1 / 2$ & $<1.95$ & $<1.82$ & $<1.89$ & & & \\
\hline \$95T003284 & \multirow[t]{2}{*}{$114: 3$} & Upper $1 / 2$ & $<1.98$ & $<1.89$ & $<1.94$ & & & \\
\hline S95T003285 & & Lower $1 / 2$ & $<16.9$ & $<19.3$ & $<18.1$ & & & \\
\hline S95T003286 & \multirow[t]{2}{*}{$114: 4$} & Upper $1 / 2$ & $<2.23$ & $<1.97$ & $<2.10$ & & & \\
\hline S95T003287 & & Lower $1 / 2$ & $<3.51$ & $<4.43$ & $<3.97$ & & & \\
\hline \$95T003288 & \multirow[t]{2}{*}{$114: 5$} & Upper $1 / 2$ & $<2.22$ & $<1.93$ & $<2.08$ & & & \\
\hline S95T003289 & & Lower $1 / 2$ & 4.10 & $<2.34$ & 3.22 & & & \\
\hline \$95T003290 & \multirow[t]{2}{*}{$114: 6$} & Upper $1 / 2$ & $<2.26$ & $<1.98$ & $<2.12$ & & & \\
\hline S95T003291 & & Lower $1 / 2$ & $<3.44$ & $<4.25$ & $<3.85$ & & & \\
\hline S95T003292 & \multirow[t]{2}{*}{$114: 7$} & Upper $1 / 2$ & $<2.34$ & $<2.17$ & $<2.23$ & & & \\
\hline S95T003293 & & Lower 1/2 & $<2.16$ & $<1.94$ & $<2.05$ & & & \\
\hline S95T003294 & \multirow[t]{2}{*}{$114: 8$} & Upper $1 / 2$ & $<2.17$ & $<3.58$ & $<2.88$ & & & \\
\hline S95T003295 & & Lower $1 / 2$ & $<2.05$ & $<2.00$ & $<2.03$ & & & \\
\hline \$95T003296 & \multirow[t]{2}{*}{$114: 9$} & Upper $1 / 2$ & $<4.11$ & $<4.64$ & $<4.38$ & & & \\
\hline S95T003297 & & Lower $1 / 2$ & $<3.79$ & $<4.50$ & $<4.15$ & & & \\
\hline
\end{tabular}


Table B-36. Tank 241-B-204 Analytical Results: Zirconium. (3 sheets)

\begin{tabular}{|c|c|c|c|c|c|c|c|c|}
\hline oning & 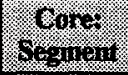 & (3) & Aran & binllant & yon. & 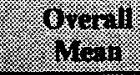 & 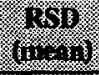 & 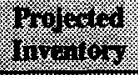 \\
\hline Solds: & & & 189 & 1.jy & (1) & 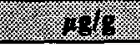 & 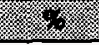 & 193 \\
\hline S95T003298 & \multirow[t]{2}{*}{$114: 10$} & Upper $1 / 2$ & $<3.70$ & $<3.59$ & $<3.65$ & & & \\
\hline S95T003299 & & Lower $1 / 2$ & $<3.39$ & $<3.85$ & $<3.62$ & & & \\
\hline S95T003300 & \multirow[t]{2}{*}{$114: 11$} & Upper $1 / 2$ & $<4.70$ & $<4.83$ & $<4.76$ & & & \\
\hline S95T003301 & & Lower $1 / 2$ & $<3.40$ & $<3.40$ & $<3.40$ & & & \\
\hline S95T003302 & \multirow[t]{2}{*}{$114: 12$} & Upper $1 / 2$ & $<2.40$ & $<2.21$ & $<2.31$ & & & \\
\hline S95T003303 & & Lower $1 / 2$ & $<2.23$ & $<3.70$ & $<2.97$ & & & \\
\hline S95T003304 & \multirow[t]{2}{*}{ 114:13 } & Upper $1 / 2$ & $<4.58$ & $<4.09$ & $<4.34$ & & & \\
\hline S95T003305 & & Lower $1 / 2$ & $<3.60$ & $<3.66$ & $<3.36$ & & & \\
\hline S95T003306 & \multirow[t]{2}{*}{$114: 14$} & Upper $1 / 2$ & $<4.29$ & $<4.27$ & $<4.28$ & & & \\
\hline \$95T003307 & & Lower $1 / 2$ & $<3.86$ & $<4.22$ & $<4.04$ & & & \\
\hline \multicolumn{3}{|c|}{ Gulatile riguis } & 191.11 & $.19 f$ min & 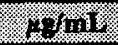 & $(181111$ & X. & 18 \\
\hline S95T002976 & 112:1 & DL & $<2.01$ & $<2.01$ & $<2.01$ & \multirow[t]{3}{*}{$<1.51$} & \multirow[t]{3}{*}{$n / a$} & \multirow[t]{3}{*}{$<0.00604$} \\
\hline S95T002984 & $112: 2$ & $\mathrm{DL}$ & $<2.01$ & $<2.01$ & $<2.01$ & & & \\
\hline \$95T003255 & $114: 1$ & $\mathrm{DL}$ & $<1.01$ & $<1.01$ & $<1.01$ & & & \\
\hline
\end{tabular}


Table B-37. Tank 241-B-204 Analytical Results: Chloride. (3 sheets)

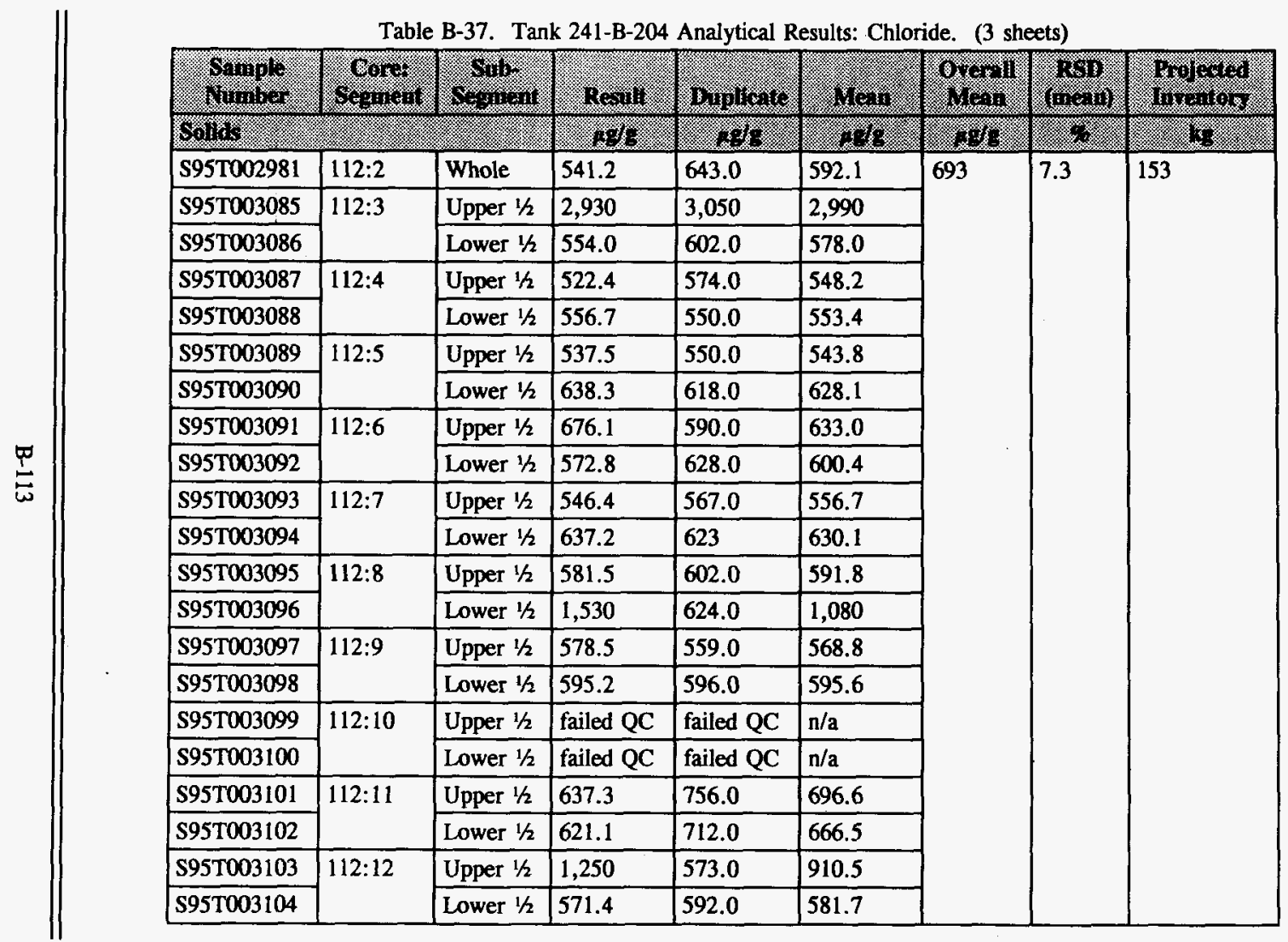




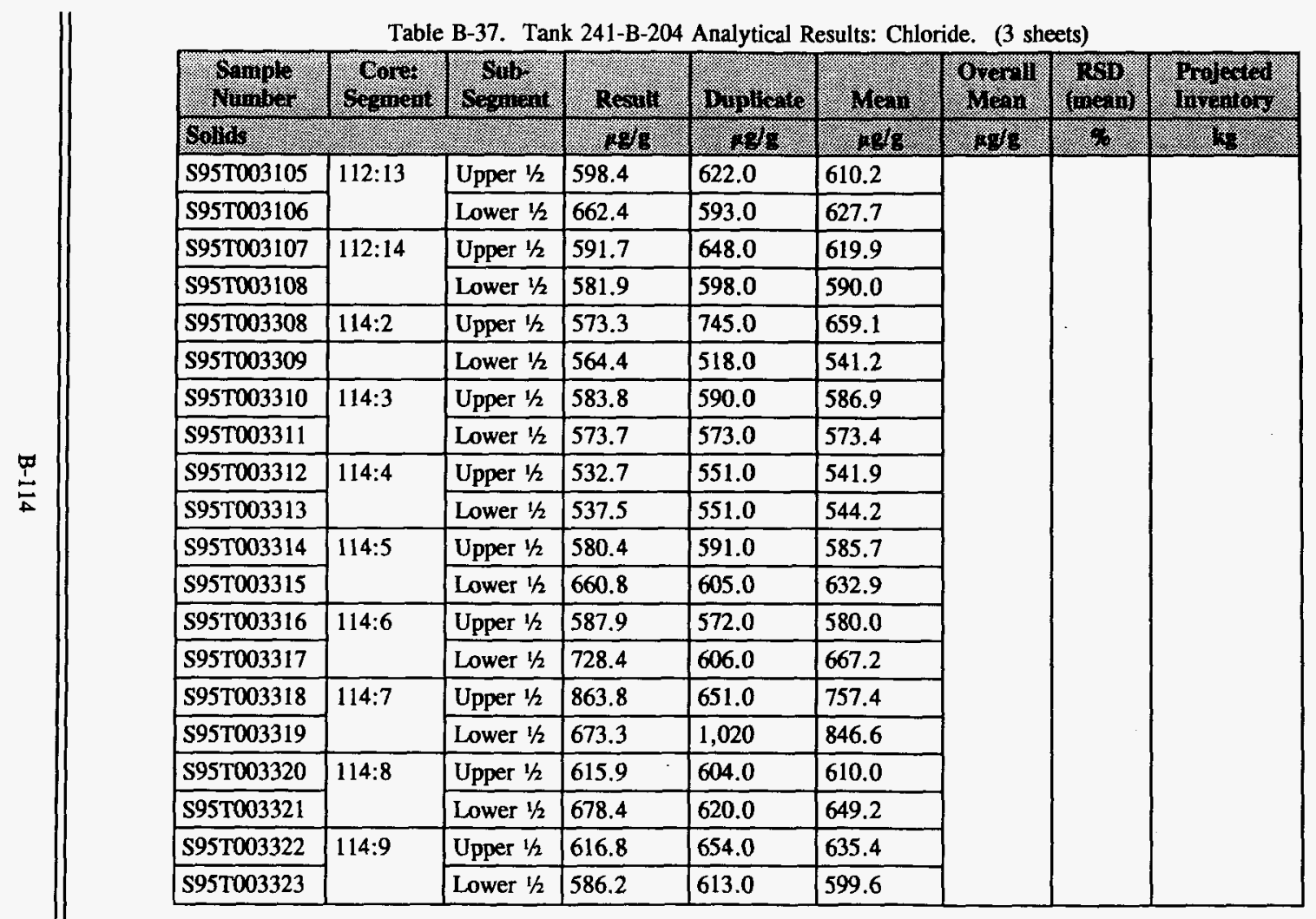


Table B-37. Tank 241-B-204 Analytical Results: Chloride. (3 sheets)

\begin{tabular}{|c|c|c|c|c|c|c|c|c|}
\hline Whing & $\left(x_{0}\right)$ & 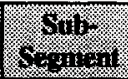 & hisk & 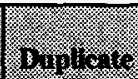 & ing & 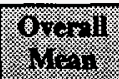 & $\left(\begin{array}{l}101) \\
(13 \times 13)\end{array}\right.$ & 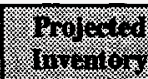 \\
\hline Sorin & & (18: & 要 & . & 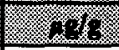 & 30 & 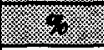 & (r) \\
\hline S95T003324 & \multirow[t]{2}{*}{$114: 10$} & Upper $1 / 2$ & 658.9 & 625.0 & 642.0 & & & \\
\hline \$95T003325 & & Lower $1 / 2$ & 614.8 & 639.0 & 626.9 & & & \\
\hline S95T003326 & \multirow[t]{2}{*}{ 114:11 } & Upper $1 / 2$ & 642.0 & 773.0 & 707.5 & & & \\
\hline S95T003327 & & Lower $1 / 2$ & 630.7 & 1,180 & 905.4 & & & \\
\hline S95T003328 & \multirow[t]{2}{*}{$114: 12$} & Upper $1 / 2$ & 675.7 & 657.0 & 666.4 & & & \\
\hline S95T003329 & & Lower $1 / 2$ & 647.6 & 647.0 & 647.3 & & & \\
\hline S95T003330 & \multirow[t]{2}{*}{$114: 13$} & Upper $1 / 2$ & 460.3 & 1,370 & 915.1 & & & \\
\hline S95T003331 & & Lower $1 / 2$ & 611.2 & 606.0 & 608.6 & & & \\
\hline S95T003332 & \multirow[t]{2}{*}{ 114:14 } & Upper $1 / 2$ & 602.3 & 613.0 & 607.6 & & & \\
\hline S95T003333 & & Lower $1 / 2$ & 672.1 & 622.0 & 647.0 & & & \\
\hline \multicolumn{3}{|c|}{ Drainble Galds } & 25111 & 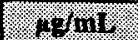 & 4. & $2 \% / \mathrm{mis}$ & $\gamma$ & 48 \\
\hline S95T002976 & $112: 1$ & $\mathrm{DL}$ & 691.2 & 670.0 & 680.6 & \multirow[t]{3}{*}{592} & \multirow[t]{3}{*}{8.8} & \multirow[t]{3}{*}{2.37} \\
\hline S95T002984 & $112: 2$ & $\mathrm{DL}$ & 596.9 & 620.0 & 608.5 & & & \\
\hline S95T003255 & $114: 1$ & DL & 556.1 & 522.0 & 539.0 & & & \\
\hline
\end{tabular}




\begin{tabular}{|c|c|c|c|c|c|c|c|c|}
\hline Goling & 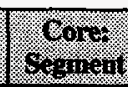 & $x^{2}$ & (3) & 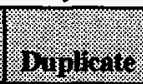 & 4 & OHe & (a) & 40 \\
\hline Solns: & 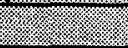 & l. & . & 47 & 4.48 & 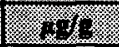 & . & (2) \\
\hline S95T002981 & 112:2 & Whole & 6,470 & 7,490 & 6,980 & \multirow[t]{21}{*}{7,080} & \multirow[t]{21}{*}{9.5} & \multirow[t]{21}{*}{1,560} \\
\hline S95T003085 & \multirow[t]{2}{*}{$112: 3$} & Upper $1 / 2$ & 35,300 & 35,000 & 35,200 & & & \\
\hline S95T003086 & & Lower $1 / 2$ & 6,390 & 7,090 & 6,740 & & & \\
\hline S95T003087 & \multirow[t]{2}{*}{$112: 4$} & Upper $1 / 2$ & 4,930 & dup error & 4,930 & & & \\
\hline S95T003088 & & Lower $1 / 2$ & 6,550 & 6,280 & 6,420 & & & \\
\hline S95T003089 & \multirow[t]{2}{*}{$112: 5$} & Upper $1 / 2$ & 6,130 & 6,300 & 6,220 & & & \\
\hline S95T003090 & & Lower $1 / 2$ & failed QC & failed QC & $n / a$ & & & \\
\hline S95T003091 & \multirow[t]{2}{*}{$112: 6$} & Upper $1 / 2$ & failed QC & failed QC & $n / a$ & & & \\
\hline \$95T003092 & & Lower $1 / 2$ & 6,250 & 6,470 & 6,360 & & & \\
\hline \$95T003093 & \multirow[t]{2}{*}{$112: 7$} & Upper $1 / 2$ & 6,020 & 6,370 & 6,190 & & & \\
\hline S95T003094 & & Lower $1 / 2$ & 6,940 & 6,910 & 6,930 & & & \\
\hline S95T003095 & \multirow[t]{2}{*}{$112: 8$} & Upper $1 / 2$ & 6,480 & 6,770 & 6,620 & & & \\
\hline \$95T003096 & & Lower $1 / 2$ & failed QC & failed QC & n/a & & & \\
\hline S95T003097 & \multirow[t]{2}{*}{$112: 9$} & Upper $1 / 2$ & failed QC & failed QC & $n / a$ & & & \\
\hline S95T003098 & & Lower $1 / 2$ & 6,660 & 6,420 & 6,540 & & & \\
\hline \$95T003099 & \multirow[t]{2}{*}{$112: 10$} & Upper $1 / 2$ & failed QC & failed QC & $n / a$ & & & \\
\hline S95T003100 & & Lower $1 / 2$ & failed QC & failed QC & $n / a$ & & & \\
\hline S95T003101 & \multirow[t]{2}{*}{$112: 11$} & Upper $1 / 2$ & 6,110 & 6,140 & 6,120 & & & \\
\hline \$95T003102 & & Lower $1 / 2$ & 5,920 & 6,150 & 6,030 & & & \\
\hline S95T003103 & \multirow[t]{2}{*}{$112: 12$} & Upper $1 / 2$ & 6,120 & 6,160 & 6,140 & & & \\
\hline S95T003104 & & Lower $1 / 2$ & 6,010 & 6,120 & 6,060 & & & \\
\hline
\end{tabular}


Table B-38. Tank 241-B-204 Analytical Results: Fluoride. (3 sheets)

\begin{tabular}{|c|c|c|c|c|c|c|c|c|}
\hline \% & 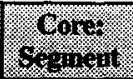 & 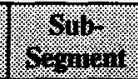 & : & B.tinge & 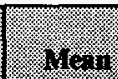 & 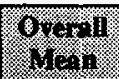 & 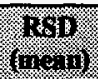 & W\% \\
\hline \multicolumn{3}{|l|}{ Solins. } & 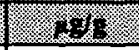 & 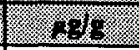 & 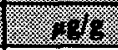 & 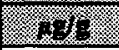 & $\sqrt{3}, 3=$ & (1) \\
\hline S95T003105 & \multirow[t]{2}{*}{$112: 13$} & Upper $1 / 2$ & 6,060 & 6,540 & 6,570 & & & \\
\hline S95T003106 & & Lower $1 / 2$ & 6,830 & 6,480 & 6,660 & & & \\
\hline S95T003107 & \multirow[t]{2}{*}{ 112:14 } & Upper $1 / 2$ & 6,390 & 6,750 & 6,570 & & & \\
\hline S95T003108 & & Lower $1 / 2$ & 6,460 & 6,540 & 6,500 & & & \\
\hline S95T003308 & \multirow[t]{2}{*}{$114: 2$} & Upper $1 / 2$ & 6,000 & 6,800 & 6,400 & & & \\
\hline S95T003309 & & Lower $1 / 2$ & 6,260 & 5,490 & 5,880 & & & \\
\hline S95T003310 & \multirow[t]{2}{*}{$114: 3$} & Upper $1 / 2$ & failed QC & failed QC & $n / a$ & & & \\
\hline S95T003311 & & Lower $1 / 2$ & failed QC & failed QC & $n / a$ & & & \\
\hline S95T003312 & \multirow[t]{2}{*}{$114: 4$} & Upper $1 / 2$ & 6,270 & 6,190 & 6,230 & & & \\
\hline S95T003313 & & Lower $1 / 2$ & 6,390 & 6,410 & 6,400 & & & \\
\hline S95T003314 & \multirow[t]{2}{*}{$114: 5$} & Upper $1 / 2$ & 5,550 & 5,480 & 5,520 & & & \\
\hline S95T003315 & & Lower $1 / 2$ & 5,830 & 5,860 & 5,840 & & & \\
\hline S95T003316 & \multirow[t]{2}{*}{$114: 6$} & Upper $1 / 2$ & 6,370 & 6,080 & 6,230 & & & \\
\hline \$95T003317 & & Lower $1 / 2$ & 6,560 & 6,570 & 6,570 & & & \\
\hline S95T003318 & \multirow[t]{2}{*}{$114: 7$} & Upper $1 / 2$ & 5,720 & 6,680 & 6,200 & & & \\
\hline S95T003319 & & Lower $1 / 2$ & 6,960 & 6,980 & 6,970 & & & \\
\hline \$95T003320 & \multirow[t]{2}{*}{$114: 8$} & Upper $1 / 2$ & 7,010 & 6,890 & 6,950 & & & \\
\hline S95T003321 & & Lower $1 / 2$ & 6,580 & 6,810 & 6,700 & & & \\
\hline S95T003322 & \multirow[t]{2}{*}{$114: 9$} & Upper $1 / 2$ & 6,670 & 6,840 & 6,760 & & & \\
\hline S95T003323 & & Lower $1 / 2$ & 6,680 & 6,490 & 6,590 & & & \\
\hline
\end{tabular}


Table B-38. Tank 241-B-204 Analytical Results: Fluoride. (3 sheets)

\begin{tabular}{|c|c|c|c|c|c|c|c|c|}
\hline 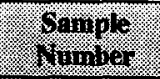 & 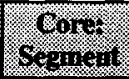 & 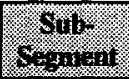 & $(2, s+1)$ & 3) & $18 \%$ & 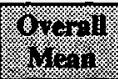 & $\frac{1010}{(1+2) 111}$ & 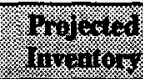 \\
\hline \multicolumn{3}{|l|}{ (6) } & $\sqrt{3}+8$ & 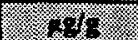 & (1) & tos & 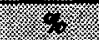 & 2 \\
\hline S95T003324 & \multirow[t]{2}{*}{$114: 10$} & Upper $1 / 2$ & 6,980 & 6,870 & 6,930 & & & \\
\hline S95T003325 & & Lower $1 / 2$ & 6,710 & 6,790 & 6,750 & & & \\
\hline S95T003326 & \multirow[t]{2}{*}{$114: 11$} & Upper $1 / 2$ & 6,830 & 7,520 & 7,180 & & & \\
\hline S95T003327 & & Lower $1 / 2$ & 6,740 & 7,030 & 6,890 & & & \\
\hline S95T003328 & \multirow[t]{2}{*}{$114: 12$} & Upper $1 / 2$ & 6,860 & 7,040 & 6,950 & & & \\
\hline S95T003329 & & Lower $1 / 2$ & 7,090 & 6,990 & 7,040 & & & \\
\hline S95T003330 & \multirow[t]{2}{*}{$114: 13$} & Upper $1 / 2$ & 6,520 & 6,730 & 6,630 & & & \\
\hline S95T003331 & & Lower $1 / 2$ & 6,800 & 6,740 & 6,770 & & & \\
\hline S95T003332 & \multirow[t]{2}{*}{$114: 14$} & Upper $1 / 2$ & 6,390 & 6,380 & 6,390 & & & \\
\hline S95T003333 & & Lower $1 / 2$ & 6,330 & 6,590 & 6,460 & & & \\
\hline \multicolumn{3}{|c|}{ Braminole Houlds } & 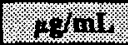 & 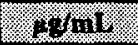 & . 1111 & 1\%olit: & 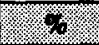 & 43 \\
\hline S95T002976 & $112: 1$ & $\mathrm{DL}$ & 6,840 & 6,700 & 6,770 & \multirow[t]{3}{*}{6,240} & \multirow[t]{3}{*}{6.0} & \multirow[t]{3}{*}{25.0} \\
\hline S95T002984 & $112: 2$ & $\overline{\mathrm{DL}}$ & 6,460 & 6,480 & 6,470 & & & \\
\hline S95T003255 & $114: 1$ & $\mathrm{DL}$ & 6,100 & 5,630 & 5,860 & & & \\
\hline
\end{tabular}




\begin{tabular}{|c|c|c|c|c|c|c|c|c|}
\hline 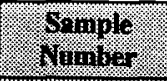 & 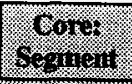 & 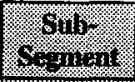 & gesing & 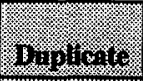 & 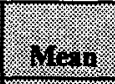 & 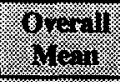 & 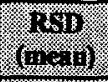 & 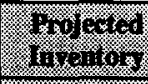 \\
\hline andolos. & . & (3) & 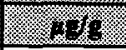 & 34 & 18 & $2 y$ & 壮 & (1) \\
\hline S95T002981 & 112:2 & Whole & 40,600 & 49,200 & 44,900 & \multirow[t]{21}{*}{52,900} & \multirow[t]{21}{*}{7.4} & \multirow[t]{21}{*}{11,600} \\
\hline S95T003085 & \multirow[t]{2}{*}{$112: 3$} & Upper $1 / 2$ & 246,000 & 244,000 & 245,000 & & & \\
\hline S95T003086 & & Lower $1 / 2$ & 46,800 & 51,700 & 49,200 & & & \\
\hline S95T003087 & \multirow[t]{2}{*}{$112: 4$} & Upper $1 / 2$ & 35,600 & dup error & 35,600 & & & \\
\hline S95T003088 & & Lower $1 / 2$ & 49,400 & 47,900 & 48,600 & & & \\
\hline S95T003089 & \multirow[t]{2}{*}{$112: 5$} & Upper $1 / 2$ & 47,300 & 48,200 & 47,800 & & & \\
\hline S95T003090 & & Lower $1 / 2$ & 53,600 & 51,300 & 52,400 & & & \\
\hline S95T003091 & \multirow[t]{2}{*}{$112: 6$} & Upper $1 / 2$ & 58,800 & 51,500 & 55,100 & & & \\
\hline S95T003092 & & Lower $1 / 2$ & 49,900 & 50,500 & 50,200 & & & \\
\hline S95T003093 & \multirow[t]{2}{*}{$112: 7$} & Upper $1 / 2$ & 47,300 & 49,400 & 48,300 & & & \\
\hline S95T003094 & & Lower $1 / 2$ & 54,500 & 53,900 & 54,200 & & & \\
\hline S95T003095 & \multirow[t]{2}{*}{$112: 8$} & Upper $1 / 2$ & 51,100 & 53,100 & 52,100 & & & \\
\hline S95T003096 & & Lower $1 / 2$ & 51,900 & 53,600 & 52,800 & & & \\
\hline S95T003097 & \multirow[t]{2}{*}{$112: 9$} & Upper $1 / 2$ & 51,100 & 49,300 & 50,200 & & & \\
\hline S95T003098 & & Lower $1 / 2$ & 51,900 & 50,200 & 51,000 & & & \\
\hline S95T003099 & \multirow[t]{2}{*}{$112: 10$} & Upper $1 / 2$ & 46,200 & 47,500 & 46,800 & & & \\
\hline \$95T003100 & & Lower $1 / 2$ & 51,400 & 51,500 & 51,500 & & & \\
\hline \$95T003101 & \multirow[t]{2}{*}{$112: 11$} & Upper $1 / 2$ & 47,700 & 45,300 & 46,500 & & & \\
\hline S95T003102 & & Lower $1 / 2$ & 43,200 & 45,200 & 44,200 & & & \\
\hline s95T003103 & \multirow[t]{2}{*}{$112: 12$} & Upper $1 / 2$ & 49,000 & 48,000 & 48,500 & & & \\
\hline S95T003104 & & Lower $1 / 2$ & 46,700 & 48,000 & 47,300 & & & \\
\hline
\end{tabular}


Table B-39. Tank 241-B-204 Analytical Results: Nitrate. (3 sheets)

\begin{tabular}{|c|c|c|c|c|c|c|c|c|}
\hline 6ring: & 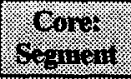 & 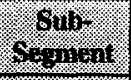 & (mosin) & Bingr, & (1) & Hor & 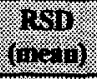 & Whow \\
\hline golg & & & 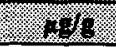 & 18 & 18 & 㳔 & $1 \%$ & (6) \\
\hline S95T003105 & \multirow[t]{2}{*}{$112: 13$} & Upper $1 / 2$ & 50,700 & 50,900 & 50,800 & & & \\
\hline \$95T003106 & & Lower $1 / 2$ & 53,200 & 49,100 & 51,100 & & & \\
\hline S95T003107 & \multirow[t]{2}{*}{$112: 14$} & Upper $1 / 2$ & 49,700 & 51,200 & 50,500 & & & \\
\hline S95T003108 & & Lower $1 / 2$ & 48,600 & 49,400 & 49,000 & & & \\
\hline S95T003308 & \multirow[t]{2}{*}{$114: 2$} & Upper $1 / 2$ & 40,300 & 44,700 & 42,500 & & & \\
\hline S95T003309 & & Lower $1 / 2$ & 44,200 & 40,000 & 42,100 & & & \\
\hline S95T003310 & \multirow[t]{2}{*}{$114: 3$} & Upper $1 / 2$ & 47,000 & 48,000 & 47,500 & & & \\
\hline S95T003311 & & Lower $1 / 2$ & 44,600 & 46,700 & 45,600 & & & \\
\hline S95T003312 & \multirow[t]{2}{*}{$114: 4$} & Upper $1 / 2$ & 48,400 & 47,800 & 48,100 & & & \\
\hline S95T003313 & & Lower $1 / 2$ & 46,500 & 47,700 & 47,100 & & & \\
\hline S95T003314 & \multirow[t]{2}{*}{$114: 5$} & Upper $1 / 2$ & 40,400 & 40,100 & 40,200 & & & \\
\hline S95T003315 & & Lower $1 / 2$ & 42,500 & 43,000 & 42,700 & & & \\
\hline S95T003316 & \multirow[t]{2}{*}{$114: 6$} & Upper $1 / 2$ & 47,000 & 45,500 & 46,300 & & & \\
\hline S95T003317 & & Lower $1 / 2$ & 48,500 & 47,700 & 48,100 & & & \\
\hline S95T003318 & \multirow[t]{2}{*}{$114: 7$} & Upper $1 / 2$ & 44,700 & 52,400 & 48,500 & & & \\
\hline S95T003319 & & Lower $1 / 2$ & 52,400 & 53,500 & 53,000 & & & \\
\hline S95T003320 & \multirow[t]{2}{*}{$114: 8$} & Upper $1 / 2$ & 53,500 & 53,500 & 53,500 & & & \\
\hline S95T003321 & & Lower $1 / 2$ & 52,200 & 52,300 & 52,300 & & & \\
\hline \$95T003322 & \multirow[t]{2}{*}{$114: 9$} & Upper $1 / 2$ & 51,900 & 53,900 & 52,900 & & & \\
\hline S95T003323 & & Lower $1 / 2$ & 52,300 & 50,900 & 51,600 & & & \\
\hline
\end{tabular}




\begin{tabular}{|c|c|c|c|c|c|c|c|c|}
\hline 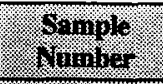 & 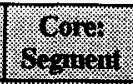 & 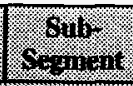 & mening & 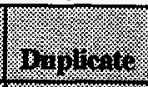 & 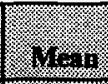 & 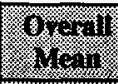 & $\frac{1+181}{(19 \%)}$ & 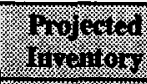 \\
\hline $6 \% 1 \%$ \% & \multirow{3}{*}{$114: 10$} & & $\frac{1}{16}$ & 18 & 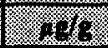 & (1) & (1) & (3) \\
\hline S95T003324 & & Upper $1 / 2$ & 54,500 & 53,800 & 54,200 & & & \\
\hline S95T003325 & & Lower $1 / 2$ & 51,900 & 52,500 & 52,200 & & & \\
\hline \$95T003326 & \multirow[t]{2}{*}{$114: 11$} & Upper $1 / 2$ & 54,300 & 58,300 & 56,300 & & & \\
\hline S95T003327 & & Lower $1 / 2$ & 53,200 & 54,900 & 54,100 & & & \\
\hline \$95T003328 & \multirow[t]{2}{*}{$114: 12$} & Upper $1 / 2$ & 53,800 & 55,400 & 54,600 & & & \\
\hline S95T003329 & & Lower $1 / 2$ & 54,800 & 54,300 & 54,600 & & & \\
\hline S95T003330 & \multirow[t]{2}{*}{$114: 13$} & Upper $1 / 2$ & 50,300 & 52,400 & 51,400 & & & \\
\hline S95T003331 & & Lower $1 / 2$ & 51,900 & 51,600 & 51,800 & & & \\
\hline S95T003332 & \multirow[t]{2}{*}{$114: 14$} & Upper $1 / 2$ & 45,200 & 45,400 & 45,300 & & & \\
\hline S95T003333 & & Lower $1 / 2$ & 45,400 & 47,500 & 46,400 & & & \\
\hline \multicolumn{3}{|c|}{ Orilinible Wquid } & 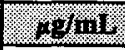 & $4 \gamma_{\mathrm{m}}$ & 28 & A $1 \mathrm{ml}$ & . & 18 \\
\hline S95T002976 & 112:1 & $\mathrm{DL}$ & 49,000 & 49,200 & 49,100 & \multirow[t]{3}{*}{45,900} & \multirow[t]{3}{*}{3.8} & \multirow[t]{3}{*}{184} \\
\hline S95T002984 & $112: 2$ & DL & 43,300 & 42,700 & 43,000 & & & \\
\hline S95T003255 & $114: 1$ & $\overline{D L}$ & 47,300 & 44,200 & 45,800 & & & \\
\hline
\end{tabular}




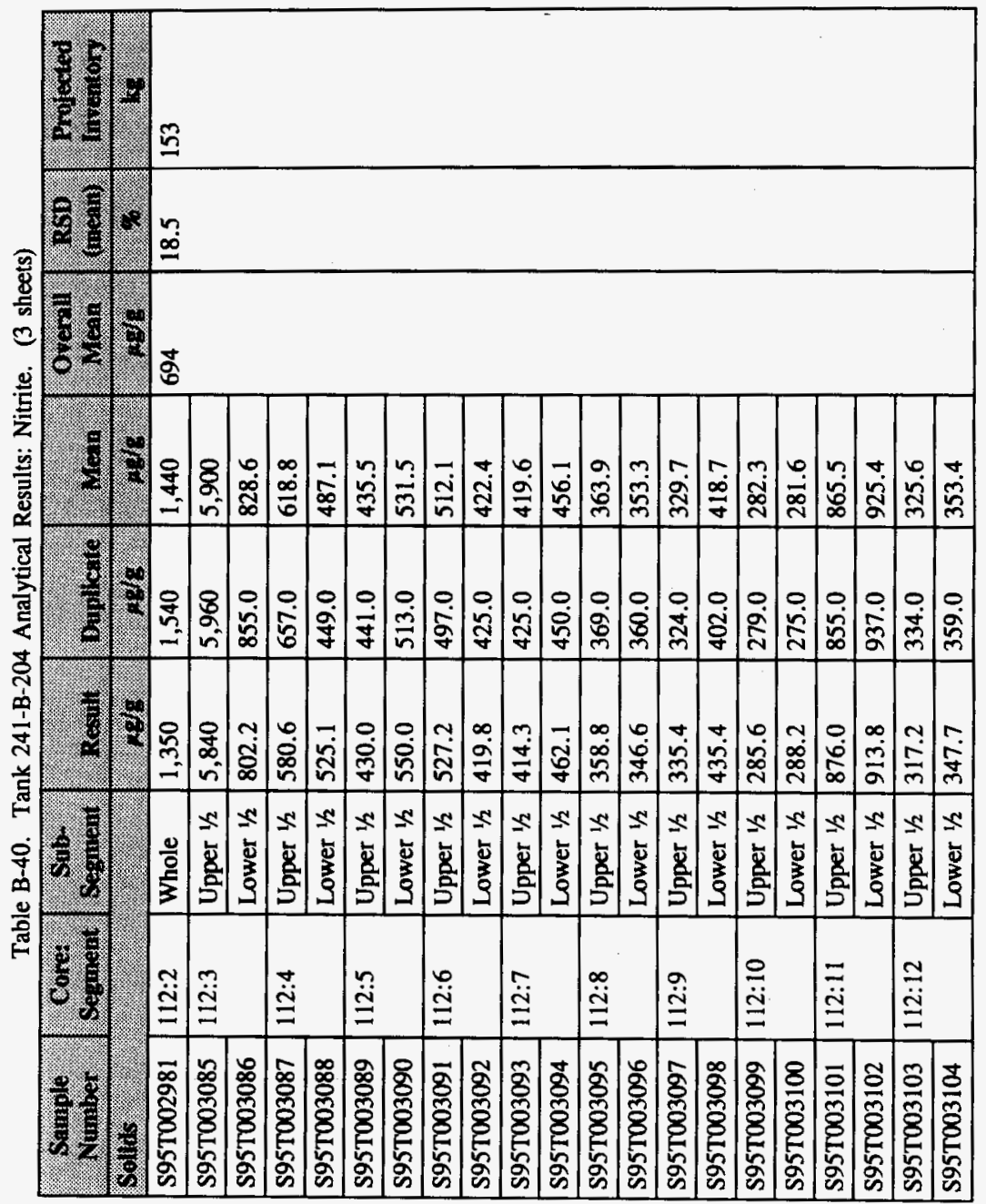


Table B-40. Tank 241-B-204 Analytical Results: Nitrite. (3 sheets)

\begin{tabular}{|c|c|c|c|c|c|c|c|c|}
\hline 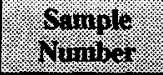 & sorits & andingin & 4 & 0,101010 & 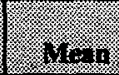 & Shor & 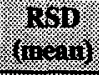 & 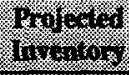 \\
\hline Solls; & & (3) & 1.95 & 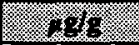 & .86 & 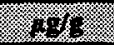 & $8 \%$ & 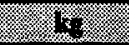 \\
\hline S95T003105 & \multirow[t]{2}{*}{ 112:13 } & Upper $1 / 2$ & 397.7 & 396.0 & 396.9 & & & \\
\hline S95T003106 & & Lower $1 / 2$ & 445.7 & 418.0 & 431.9 & & & \\
\hline S95T003107 & \multirow[t]{2}{*}{$112: 14$} & Upper $1 / 2$ & 389.2 & 386.0 & 387.6 & & & \\
\hline S95T003108 & & Lower $1 / 2$ & 406.2 & 403.0 & 404.6 & & & \\
\hline S95T003308 & \multirow[t]{2}{*}{$114: 2$} & Upper $1 / 2$ & 1,440 & 1,550 & 1,500 & & & \\
\hline S95T003309 & & Lower $1 / 2$ & 1,390 & 1,330 & 1,360 & & & \\
\hline S95T003310 & \multirow[t]{2}{*}{$114: 3$} & Upper $1 / 2$ & 1,080 & 1,080 & 1,080 & & & \\
\hline S95T003311 & & Lower $1 / 2$ & 897.8 & 901.0 & 899.4 & & & \\
\hline \$95T003312 & \multirow[t]{2}{*}{$114: 4$} & Upper $1 / 2$ & 641.0 & 644.0 & 642.5 & & & \\
\hline S95T003313 & & Lower $1 / 2$ & 825.7 & 845.0 & 835.4 & & & \\
\hline S95T003314 & \multirow[t]{2}{*}{$114: 5$} & Upper $1 / 2$ & 1,150 & 1,150 & 1,150 & & & \\
\hline S95T003315 & & Lower $1 / 2$ & 1,080 & 1,070 & 1,070 & & & \\
\hline S95T003316 & \multirow[t]{2}{*}{$114: 6$} & Upper $1 / 2$ & 1,080 & 1,070 & 1,080 & & & \\
\hline S95T003317 & & Lower $1 / 2$ & 1,160 & 1,030 & 1,100 & & & \\
\hline S95T003318 & \multirow[t]{2}{*}{$114: 7$} & Upper $1 / 2$ & 359.2 & 397.0 & 378.1 & & & \\
\hline \$95T003319 & & Lower $1 / 2$ & 478.3 & 466.0 & 472.1 & & & \\
\hline S95T003320 & \multirow[t]{2}{*}{$114: 8$} & Upper $1 / 2$ & 292.5 & 290.0 & 291.2 & & & \\
\hline S95T003321 & & Lower $1 / 2$ & 345.0 & 350.0 & 347.5 & & & \\
\hline S95T003322 & \multirow[t]{2}{*}{$114: 9$} & Upper $1 / 2$ & 351.8 & 368.0 & 359.9 & & & \\
\hline S95T003323 & & Lower $1 / 2$ & 391.3 & 382.0 & 386.6 & & & \\
\hline
\end{tabular}


Table B-40. Tank 241-B-204 Analytical Results: Nitrite. (3 sheets)

\begin{tabular}{|c|c|c|c|c|c|c|c|c|}
\hline Wingor & 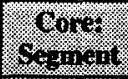 & (3) & 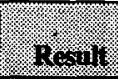 & 10146.16 & 3.1. & 筮 & $\begin{array}{l}\cos 18 \\
1+2,113\end{array}$ & 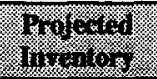 \\
\hline Soll is & (18) & rs: & 45 & 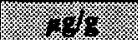 & (1) & 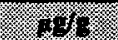 & (2) & (3) \\
\hline S95T003324 & \multirow[t]{2}{*}{$114: 10$} & Upper $1 / 2$ & 333.6 & 340.0 & 336.8 & & & \\
\hline S95T003325 & & Lower $1 / 2$ & 343.9 & 332.0 & 337.9 & & & \\
\hline S95T003326 & \multirow[t]{2}{*}{$114: 11$} & Upper $1 / 2$ & 347.3 & 351.0 & 349.1 & & & \\
\hline S95T003327 & & Lower $1 / 2$ & 343.0 & 342.0 & 342.5 & & & \\
\hline S95T003328 & \multirow[t]{2}{*}{$114: 12$} & Upper $1 / 2$ & 219.1 & 226.0 & 222.6 & & & \\
\hline S95T003329 & & Lower $1 / 2$ & 225.7 & 228.0 & 226.8 & & & \\
\hline S95T003330 & \multirow[t]{2}{*}{$114: 13$} & Upper $1 / 2$ & 932.9 & 328.0 & 630.5 & & & \\
\hline S95T003331 & & Lower $1 / 2$ & 335.0 & 340.0 & 337.5 & & & \\
\hline \$95T003332 & \multirow[t]{2}{*}{$114: 14$} & Upper $1 / 2$ & 373.2 & 398.0 & 385.6 & & & \\
\hline S95T003333 & & Lower $1 / 2$ & 381.3 & 390.0 & 385.6 & & & \\
\hline \multicolumn{3}{|c|}{ 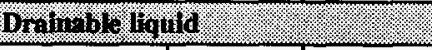 } & (10121\% & (28) & 161111 & orinis & $x$ & 4 \\
\hline S95T002976 & $112: 1$ & $\mathrm{DL}$ & 2,170 & 2,230 & 2,200 & \multirow[t]{3}{*}{1,730} & \multirow[t]{3}{*}{14.2} & \multirow[t]{3}{*}{6.92} \\
\hline S95T002984 & $112: 2$ & $\mathrm{DL}$ & 1,360 & 1,350 & 1,350 & & & \\
\hline S95T003255 & $114: 1$ & DL & 1,720 & 1,630 & 1,680 & & & \\
\hline
\end{tabular}


Table B-41. Tank 241-B-204 Analytical Results: Oxalate. (3 sheets)

\begin{tabular}{|c|c|c|c|c|c|c|c|c|}
\hline Simple & $\frac{6}{60 \%}$ & 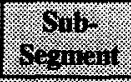 & Rosing) & 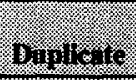 & Me:13. & \% & $\frac{1}{195}$ & 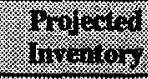 \\
\hline 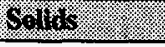 & (2) & & 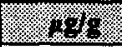 & 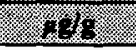 & (7) & 28 & \% & (3) \\
\hline S95T002981 & $112: 2$ & Whole & $<247$ & $<237$ & $<242$ & \multirow[t]{21}{*}{1,710} & \multirow[t]{21}{*}{5.5} & \multirow[t]{21}{*}{376} \\
\hline S95T003085 & \multirow[t]{2}{*}{$112: 3$} & Upper $1 / 2$ & 4,680 & 4,840 & 4,760 & & & \\
\hline S95T003086 & & Lower $1 / 2$ & 1,220 & 1,340 & 1,280 & & & \\
\hline S95T003087 & \multirow[t]{2}{*}{$112: 4$} & Upper $1 / 2$ & 1,330 & 1,560 & 1,450 & & & \\
\hline S95T003088 & & Lower $1 / 2$ & 1,660 & 1,580 & 1,620 & & & \\
\hline S95T003089 & \multirow[t]{2}{*}{$112: 5$} & Upper $1 / 2$ & 1,660 & 1,680 & 1,670 & & & \\
\hline S95T003090 & & Lower $1 / 2$ & 1,940 & 1,840 & 1,890 & & & \\
\hline S95T003091 & \multirow[t]{2}{*}{$112: 6$} & Upper $1 / 2$ & 2,150 & 1,880 & 2,010 & & & \\
\hline \$95T003092 & & Lower $1 / 2$ & 1,750 & 1,860 & 1,810 & & & \\
\hline S95T003093 & \multirow[t]{2}{*}{$112: 7$} & Upper $1 / 2$ & 1,700 & 1,800 & 1,750 & & & \\
\hline S95T003094 & & Lower $1 / 2$ & 2,040 & 1,940 & 1,990 & & & \\
\hline \$95T003095 & \multirow[t]{2}{*}{$112: 8$} & Upper $1 / 2$ & 1,810 & 1,920 & 1,870 & & & \\
\hline S95T003096 & & Lower $1 / 2$ & 1,780 & 1,810 & 1,790 & & & \\
\hline \$95T003097 & \multirow[t]{2}{*}{$112: 9$} & Upper $1 / 2$ & 1,700 & 1,660 & 1,680 & & & \\
\hline S95T003098 & & Lower $1 / 2$ & 1,800 & 1,760 & 1,780 & & & \\
\hline S95T003099 & \multirow[t]{2}{*}{$112: 10$} & Upper $1 / 2$ & 1,620 & 1,610 & 1,620 & & & \\
\hline S95T003100 & & Lower $1 / 2$ & 1,720 & 1,750 & 1,740 & & & \\
\hline S95T003101 & \multirow[t]{2}{*}{$112: 11$} & Upper $1 / 2$ & 2,120 & 2,110 & 2,120 & & & \\
\hline S95T003102 & & Lower $1 / 2$ & 2,120 & 2,070 & 2,100 & & & \\
\hline S95T003103 & \multirow[t]{2}{*}{$112: 12$} & Upper $1 / 2$ & 1,590 & 1,590 & 1,590 & & & \\
\hline S95T003104 & & Lower $1 / 2$ & 1,490 & 1,580 & 1,530 & & & \\
\hline
\end{tabular}




\begin{tabular}{|c|c|c|c|c|c|c|c|c|}
\hline & & & $08 L^{\prime} \mathrm{I}$ & $09 L^{\prime} I$ & $06 L^{\prime} \mathrm{I}$ & $2 / 1$ Іәмо 1 & \multirow[b]{2}{*}{$6: t I I$} & EZEع0مLS6S \\
\hline & & & $088^{6} \mathrm{I}$ & $068^{\circ} \mathrm{I}$ & $088^{\circ} \mathrm{I}$ & $y_{1 /}$ sadd $\Omega$ & & ZZEE00LS6S \\
\hline & & & $058^{\circ} \mathrm{I}$ & $058^{4} \mathrm{I}$ & $098^{6} \mathrm{I}$ & $z / 1$ Iәмо & \multirow[b]{2}{*}{$8: t 11$} & IZEE00LS6S \\
\hline & & & $0 \angle 8^{\circ} \mathrm{T}$ & $0<8^{\circ} \mathrm{I}$ & $088^{\circ} \mathrm{I}$ & $z_{1}$ Iadd $\Omega$ & & Oz\&E00LS6S \\
\hline & & & $056^{\circ} \mathrm{I}$ & $0066^{\circ} \mathrm{I}$ & $0 \angle 6^{\circ} \mathrm{I}$ & $4 / 119 \mathrm{MO}$ & \multirow[b]{2}{*}{$L: t b I I$} & 6IEE00LS6S \\
\hline & & & $069^{\circ} \mathrm{I}$ & $0+8^{\circ} \mathrm{I}$ & $0 E S^{\prime} I$ & $2 / 1$ Iədd $\Omega$ & & 8IEC00LS6S \\
\hline & & & $00 t^{\prime} 2$ & $09 \varepsilon^{\prime} z$ & $0 S t^{\prime} Z$ & \%/1 IOMOI & \multirow[b]{2}{*}{ 9:bll } & LIEE00LS6S \\
\hline & & & OIE'z & $081^{\prime} \mathrm{Z}$ & $0 S t^{\prime} 2$ & $z_{1}$ Iaddn & & 9IEE00LS6S \\
\hline & & & $011 ' 2$ & $0+0 \div 2$ & $0 L I^{\prime} Z$ & $4 / 1$ Iอм० & \multirow[b]{2}{*}{ s:tII } & SIEE00LS6S \\
\hline & & & $0 \varepsilon 0^{\prime} Z$ & $0 \varepsilon 6^{4} \mathrm{I}$ & $0+1 ' z$ & $z_{1}$ roddn & & †IEEO0LS6S \\
\hline & & & $0 S S^{\circ} \mathrm{I}$ & $0 t S^{4} I$ & $095^{\circ} \mathrm{I}$ & $2 / 1$ LәMOI & \multirow[b]{2}{*}{$t: t 11$} & EIEE00」S6S \\
\hline & & & $0 \angle Z^{\prime} I$ & OSZI I & $062^{\prime} \mathrm{I}$ & $z_{1}$ raddn & & ZIEE00LS6S \\
\hline & & & $00 \mathrm{I}^{\prime} \mathrm{I}$ & $02 I^{\prime} \mathrm{I}$ & $060^{\circ} \mathrm{I}$ & 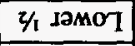 & \multirow[b]{2}{*}{$\varepsilon: t I I$} & IIEE00LS6S \\
\hline & & & $\tau^{\circ} \varepsilon+8$ & 0.588 & t.IS8 & $z_{1} \operatorname{sodd}_{\Omega}$ & & 0IEEOOLS6S \\
\hline & & & t.98t & $0 . s s t_{0}$ & L.LIS & $z / 1$ IOMO7 & \multirow[b]{2}{*}{$Z: t I I$} & 60EE00IS6S \\
\hline & & & ZIt $>$ & $0 z t>$ & $\varepsilon 0 t>$ & $\psi_{1}$ redd $\Pi$ & & 80EE00IS6S \\
\hline & & & $059^{4} \mathrm{I}$ & $069^{6} \mathrm{I}$ & $009^{\prime} I$ & $z / 129 M O 7$ & \multirow[b]{2}{*}{ DI:ZI I } & 80IE0aLS6S \\
\hline & & & $069^{\circ} \mathrm{I}$ & $0 \varepsilon L^{\prime} \mathrm{I}$ & $0+9^{\circ} \mathrm{I}$ & $4 / 1$ Iadd $n$ & & L0IE00LS6S \\
\hline & & & $0 Z L^{\prime} \mathrm{I}$ & $0+9^{6} \mathrm{I}$ & $018^{\circ} \mathrm{I}$ & $z / 12 \mathrm{MO}$ & \multirow[b]{2}{*}{ EI:ZII } & 90 I E00LS6S \\
\hline & & & $O E L^{\prime} I$ & $0 Z L^{\prime} I$ & $O E L^{\prime} I$ & $z_{1} \mathrm{I} \mathrm{dd} \Omega$ & & S0IE00LC6S \\
\hline (1) & 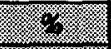 & $9 \%$ & (1/\% & 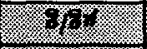 & $9 \%$ & (1) & (7) & 94102 \\
\hline 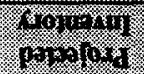 & 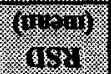 & $\begin{array}{l}0.6 \% \\
112.40\end{array}$ & $\sqrt[7]{1.41}$ & 41069 & Yist? & inowisis & 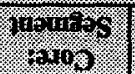 & 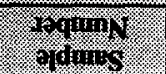 \\
\hline
\end{tabular}




\begin{tabular}{|c|c|c|c|c|c|c|c|c|}
\hline \multirow[b]{3}{*}{$2 t^{\prime} \cdot 1>$} & \multirow[b]{3}{*}{$\mathbf{B} / \mathbf{U}$} & \multirow[b]{3}{*}{$9 S \mathcal{E}>$} & $\varepsilon z \tau>$ & $\varepsilon \tau Z>$ & $\varepsilon Z Z>$ & $\mathrm{Td}$ & I:tDII & SSZE00LS6S \\
\hline & & & $\varepsilon Z 2>$ & $\mathfrak{E Z Z}>$ & $\varepsilon z Z>$ & $\mathrm{Ta}$ & Z:ZII & $\$ 86200 L S 6 S$ \\
\hline & & & $0 . \varepsilon S L$ & $0^{\circ} \varepsilon S L$ & 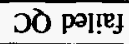 & 70 & $I:$ ZII & $9 \angle 6200 L S 6 S$ \\
\hline (1) & 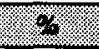 & moint: & $94 \%$ & jur & Twor: & pribivgripura & \multicolumn{2}{|c|}{ 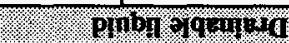 } \\
\hline & & & $0 z 8^{\prime} \mathrm{I}$ & $006^{\prime} \mathrm{I}$ & $0 \div L^{\prime} I$ & // ग्MOT & \multirow[b]{2}{*}{$t I: t I I$} & EEEE00LS6S \\
\hline & & & $0+L^{\prime} I$ & $089^{\prime} \mathrm{I}$ & $008^{6} \mathrm{I}$ & $z / 1$ sadd $n$ & & ZEEE00.L.S6S \\
\hline & & & $0 \varepsilon L^{6} I$ & OEL'I & $0 Z L^{6} \mathrm{I}$ & $2 / 1$ 12MOI & \multirow[b]{2}{*}{$\varepsilon I: t I I$} & IEEE00LS6S \\
\hline & & & $0 I L^{\prime} \mathrm{I}$ & $0 \varepsilon L^{6} I$ & $00 L^{\prime} I$ & $\psi_{1}$ Iadd $n$ & & OEEEOALSGS \\
\hline & & & $008^{\prime} \mathrm{I}$ & $0 L L^{\prime} I$ & $028^{\prime} \mathrm{I}$ & z/1 ГРMOI & \multirow[b]{2}{*}{ ZI:tII } & 6ZEE00LS6S \\
\hline & & & $098^{\prime} I$ & $088^{\prime} \mathrm{I}$ & $0 E 8^{\prime} I$ & $y_{1}$ İdd $\Lambda$ & & 8ZEE00LS6S \\
\hline & & & $028^{\prime} \mathrm{I}$ & $098^{\prime} \mathrm{I}$ & $08 L^{\prime} l$ & $2 / 1$ JOMOT & \multirow[b]{2}{*}{$I I: t I I$} & LZEEOALS6S \\
\hline & & & $026^{6} 1$ & $0 \angle 6^{\prime} \mathrm{I}$ & $098^{\prime} \mathrm{I}$ & $z / 1$ Iadd $\Omega$ & & 9ZEE00LS6S \\
\hline & & & $09 L^{\prime} I$ & OLL'I & OSLL'I & $z / 1 \mathrm{JOMOT}$ & \multirow[b]{2}{*}{ OI:DII } & SZEEOOLS6S \\
\hline & & & $088^{\prime} \mathrm{I}$ & $0 \angle 8^{\circ} \mathrm{I}$ & $088^{\prime} \mathrm{I}$ & $z_{1}$ Iadd $n$ & & tZEEOOLS6S \\
\hline 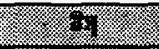 & 83 & 26 & 67 & qfor & 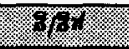 & (2) & & (1) 10 \\
\hline 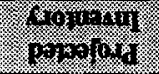 & 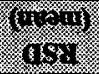 & 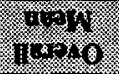 & 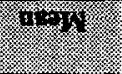 & \% & $4=24$ & monsos & 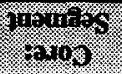 & 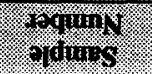 \\
\hline
\end{tabular}

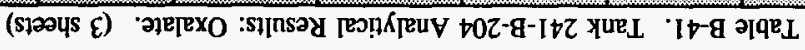




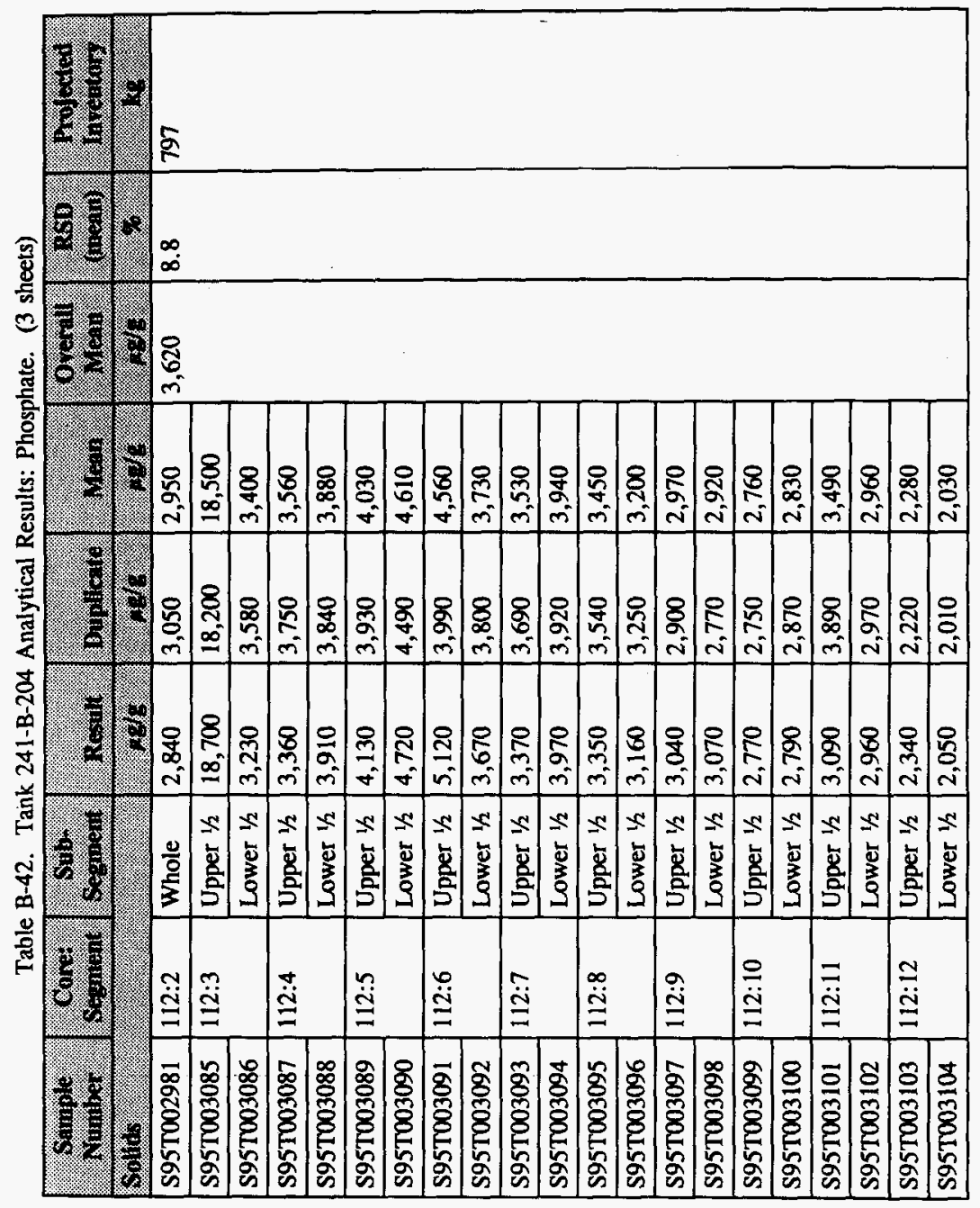




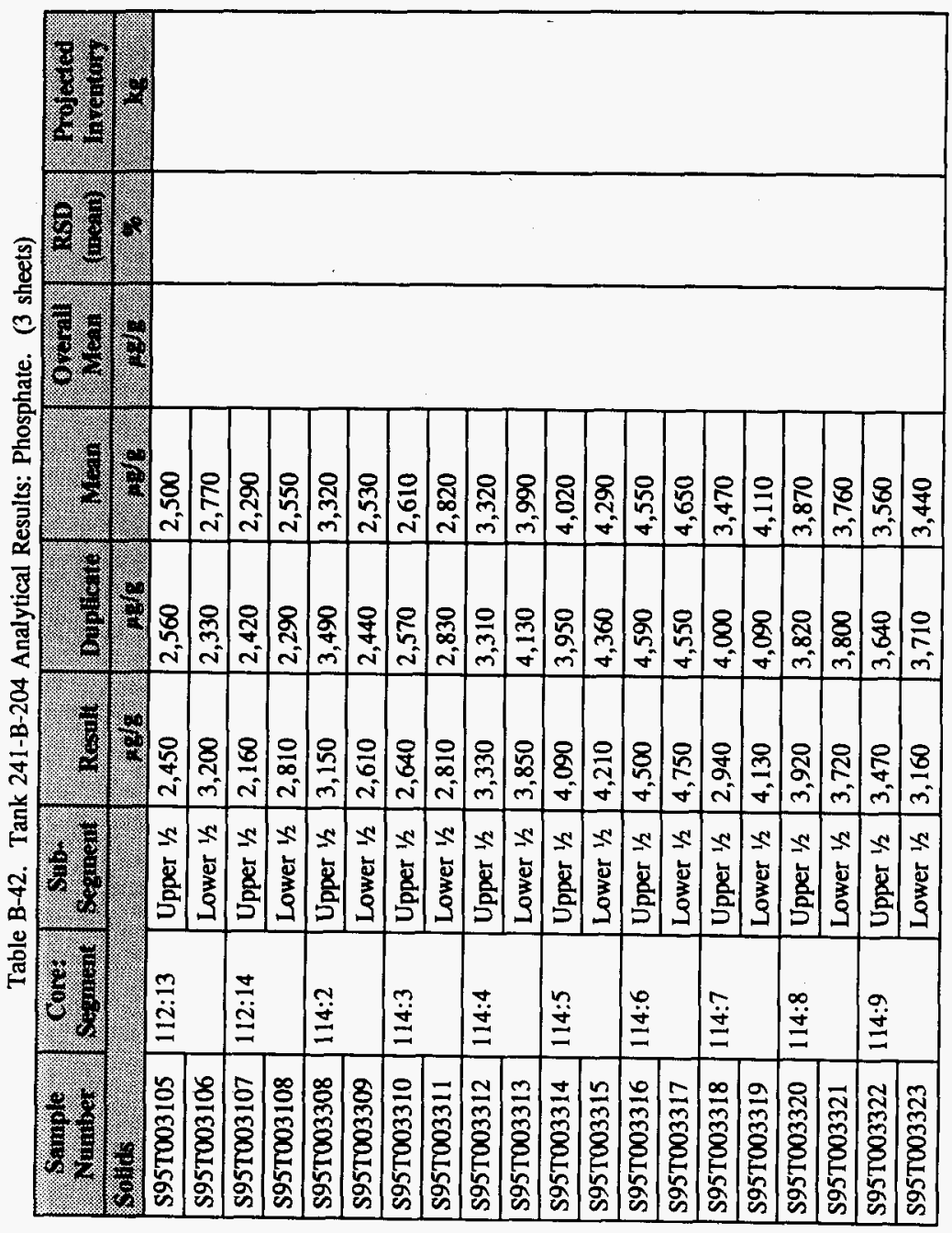


Table B-42. Tank 241-B-204 Analytical Results: Phosphate. (3 sheets)

\begin{tabular}{|c|c|c|c|c|c|c|c|c|}
\hline S4ang & (4) & 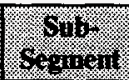 & $\sqrt{10.116}$ & Buplisilo & $x^{4}$ & $4 \%$ & $\frac{1}{10}$ & 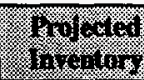 \\
\hline Solos: & 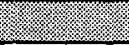 & (2) & 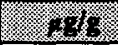 & 806 & 2.10 & 87 & 1. & 2 \\
\hline S95T003324 & \multirow[t]{2}{*}{$114: 10$} & Upper $1 / 2$ & 3,450 & 3,440 & 3,440 & & & \\
\hline S95T003325 & & Lower $1 / 2$ & 3,300 & 3,450 & 3,380 & & & \\
\hline S95T003326 & \multirow[t]{2}{*}{$114: 11$} & Upper $1 / 2$ & 3,330 & 3,590 & 3,460 & & & \\
\hline S95T003327 & & Lower $1 / 2$ & 3,200 & 3,460 & 3,330 & & & \\
\hline S95T003328 & \multirow[t]{2}{*}{$114: 12$} & Upper $1 / 2$ & 3,120 & 3,340 & 3,230 & & & \\
\hline S95T003329 & & Lower $1 / 2$ & 2,950 & 2,890 & 2,920 & & & \\
\hline S95T003330 & \multirow[t]{2}{*}{$114: 13$} & Upper $1 / 2$ & 2,730 & 3,950 & 3,340 & & & \\
\hline S95T003331 & & Lower $1 / 2$ & 2,730 & 2,750 & 2,740 & & & \\
\hline S95T003332 & \multirow[t]{2}{*}{$114: 14$} & Upper $1 / 2$ & 2,700 & 2,530 & 2,620 & & & \\
\hline S95T003333 & & Lower $1 / 2$ & 2,680 & 2,850 & 2,760 & & & \\
\hline \multicolumn{2}{|c|}{ B } & (2) & (anin & $1 / 2 \mathrm{~mL}$ & .02111 & ifoln & . & $\sqrt{18}$ \\
\hline S95T002976 & 112:1 & $\mathrm{DL}$ & 2,540 & 2,720 & 2,630 & \multirow[t]{3}{*}{2,060} & \multirow[t]{3}{*}{12.4} & \multirow[t]{3}{*}{8.24} \\
\hline S95T002984 & 112:2 & $\mathrm{DL}$ & 1,770 & 1,830 & 1,800 & & & \\
\hline S95T003255 & $114: 1$ & DL & 1,910 & 1,890 & 1,900 & & & \\
\hline
\end{tabular}


Table B-43. Tank 241-B-204 Analytical Results: Sulfate. (3 sheets)

\begin{tabular}{|c|c|c|c|c|c|c|c|c|}
\hline 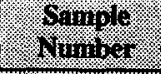 & 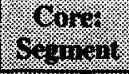 & 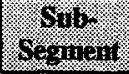 & ne-iol & $0,101,011$ & $\frac{1}{4}$ & 8\% & (nisesis) & 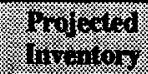 \\
\hline 40136 & & & (1) & (1) & - 116 & . & 8 & (.) \\
\hline S95T002981 & $112: 2$ & Whole & 658.9 & 715.0 & 687.0 & \multirow[t]{21}{*}{648} & \multirow[t]{21}{*}{12.0} & \multirow[t]{21}{*}{143} \\
\hline S95T003085 & \multirow[t]{2}{*}{$112: 3$} & Upper $1 / 2$ & 3,760 & 3,970 & 3,860 & & & \\
\hline S95T003086 & & Lower $1 / 2$ & 474.7 & 542.0 & 508.4 & & & \\
\hline S95T003087 & \multirow[t]{2}{*}{$112: 4$} & Upper $1 / 2$ & 419.6 & 451.0 & 435.3 & & & \\
\hline S95T003088 & & Lower $1 / 2$ & 444.2 & 421.0 & 432.6 & & & \\
\hline$\$ 95 T 003089$ & \multirow[t]{2}{*}{$112: 5$} & Upper $1 / 2$ & 415.6 & 424.0 & 419.8 & & & \\
\hline S95T003090 & & Lower $1 / 2$ & 574.2 & 614.0 & 594.1 & & & \\
\hline \$95T003091 & \multirow[t]{2}{*}{$112: 6$} & Upper $1 / 2$ & 626.0 & 536.0 & 581.0 & & & \\
\hline S95T003092 & & Lower $1 / 2$ & 463.0 & 992.0 & 727.5 & & & \\
\hline S95T003093 & \multirow[t]{2}{*}{$112: 7$} & Upper $1 / 2$ & 482.0 & 414.0 & 448.0 & & & \\
\hline \$95T003094 & & Lower $1 / 2$ & 474.6 & 487.0 & 480.8 & & & \\
\hline S95T003095 & \multirow[t]{2}{*}{$112: 8$} & Upper $1 / 2$ & 347.0 & 427.0 & 387.0 & & & \\
\hline S95T003096 & & Lower $1 / 2$ & 532.8 & 411.0 & 471.9 & & & \\
\hline S95T003097 & \multirow[t]{2}{*}{$112: 9$} & Upper $1 / 2$ & 378.6 & 333.0 & 355.8 & & & \\
\hline S95T003098. & & Lower $1 / 2$ & 478.5 & 406.0 & 442.2 & & & \\
\hline S95T003099 & \multirow[t]{2}{*}{$112: 10$} & Upper $1 / 2$ & 281.3 & 283.0 & 282.1 & & & \\
\hline S95T003100 & & Lower $1 / 2$ & 265.1 & 810.0 & 537.5 & & & \\
\hline S95T003101 & \multirow[t]{2}{*}{ 112:11 } & Upper $1 / 2$ & 986.8 & 936.0 & 961.4 & & & \\
\hline S95T003102 & & Lower $1 / 2$ & 961.7 & 1,360 & 1,160 & & & \\
\hline S95T003103 & \multirow[t]{2}{*}{$112: 12$} & Upper $1 / 2$ & 357.4 & 377.0 & 367.2 & & & \\
\hline S95T003104 & & Lower $1 / 2$ & 500.6 & 418.0 & 459.3 & & & \\
\hline
\end{tabular}


Table B-43. Tank 241-B-204 Analytical Results: Sulfate. (3 sheets)

\begin{tabular}{|c|c|c|c|c|c|c|c|c|}
\hline oiniols & 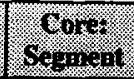 & 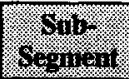 & 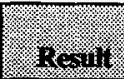 & 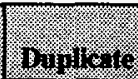 & $41+1$. & 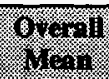 & $\begin{array}{l}\cos 85 \\
(19+1)\end{array}$ & Wrisers \\
\hline $10113 \%$ & & (2) & 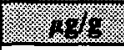 & 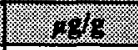 & 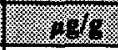 & 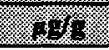 & ( & r. \\
\hline S95T003105 & \multirow[t]{2}{*}{$112: 13$} & Upper $1 / 2$ & 479.5 & 439.0 & 459.2 & & & \\
\hline S95T003106 & & Lower $1 / 2$ & 540.5 & 463.0 & 501.8 & & & \\
\hline \$95T003107 & \multirow[t]{2}{*}{$112: 14$} & Upper $1 / 2$ & 454.0 & 446.0 & 450.0 & & & \\
\hline S95T003108 & & Lower $1 / 2$ & 513.6 & 500.0 & 506.8 & & & \\
\hline S95T003308 & \multirow[t]{2}{*}{$114: 2$} & Upper $1 / 2$ & 813.5 & 875.0 & 844.2 & & & \\
\hline S95T003309 & & Lower $1 / 2$ & 1,020 & 1,080 & 1,050 & & & \\
\hline S95T003310 & \multirow[t]{2}{*}{$114: 3$} & Upper $1 / 2$ & 613.4 & 681.0 & 647.2 & & & \\
\hline S95T003311 & & Lower $1 / 2$ & 568.5 & 584.0 & 576.2 & & & \\
\hline S95T003312 & \multirow[t]{2}{*}{$114: 4$} & Upper $1 / 2$ & 436.0 & 431.0 & 433.5 & & & \\
\hline \$95T003313 & & Lower $1 / 2$ & 735.2 & 791.0 & 763.1 & & & \\
\hline S95T003314 & \multirow[t]{2}{*}{$114: 5$} & Upper $1 / 2$ & 1,110 & 1,220 & 1,160 & & & \\
\hline S95T003315 & & Lower $1 / 2$ & 1,140 & 1,040 & 1,090 & & & \\
\hline S95T003316 & \multirow[t]{2}{*}{$114: 6$} & Upper $1 / 2$ & 1,200 & 1,160 & 1,180 & & & \\
\hline S95T003317 & & Lower $1 / 2$ & 1,120 & 1,100 & 1,110 & & & \\
\hline S95T003318 & \multirow[t]{2}{*}{$114: 7$} & Upper $1 / 2$ & 511.6 & 484.0 & 497.8 & & & \\
\hline S95T003319 & & Lower $1 / 2$ & 599.8 & 714.0 & 656.9 & & & \\
\hline S95T003320 & \multirow[t]{2}{*}{$114: 8$} & Upper $1 / 2$ & 533.4 & 469.0 & 501.2 & & & \\
\hline S95T003321 & & Lower $1 / 2$ & 423.4 & 987.0 & 705.2 & & & \\
\hline S95T003322 & \multirow[t]{2}{*}{$114: 9$} & Upper $1 / 2$ & 449.6 & 464.0 & 456.8 & & & \\
\hline S95T003323 & & Lower $1 / 2$ & 468.3 & 481.0 & 474.6 & & & \\
\hline
\end{tabular}


Table B-43. Tank 241-B-204 Analytical Results: Sulfate. (3 sheets)

\begin{tabular}{|c|c|c|c|c|c|c|c|c|}
\hline 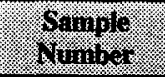 & 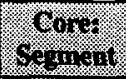 & ong & as $=1$ & Diaplients & $1=-118$ & 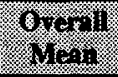 & 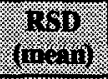 & W \\
\hline sotints & & & 29 & 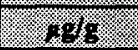 & 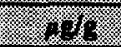 & (1) & (x) & $\sqrt{1.3}$ \\
\hline S95T003324 & \multirow[t]{2}{*}{$114: 10$} & Upper $1 / 2$ & 422.3 & 420.0 & 421.0 & & & \\
\hline \$95T003325 & & Lower $1 / 2$ & 452.9 & 355.0 & 403.9 & & & \\
\hline S95T003326 & \multirow[t]{2}{*}{$114: 11$} & Upper $1 / 2$ & 433.2 & 420.0 & 426.6 & & & \\
\hline S95T003327 & & Lower $1 / 2$ & 990.2 & 465.0 & 727.6 & & & \\
\hline S95T003328 & \multirow[t]{2}{*}{$114: 12$} & Upper $1 / 2$ & 318.4 & 301.0 & 309.7 & & & \\
\hline S95T003329 & & Lower $1 / 2$ & 279.0 & 296.0 & 287.5 & & & \\
\hline S95T003330 & \multirow[t]{2}{*}{$114: 13$} & Upper $1 / 2$ & 413.2 & 542.0 & 477.6 & & & \\
\hline \$95T003331 & & Lower $1 / 2$ & 401.1 & 413.0 & 407.1 & & & \\
\hline S95T003332 & \multirow[t]{2}{*}{$114: 14$} & Upper $1 / 2$ & 419.7 & 480.0 & 449.9 & & & \\
\hline S95T003333 & & Lower $1 / 2$ & 432.2 & 474.0 & 453.1 & & & \\
\hline \multicolumn{3}{|c|}{ Dosingbe lourd } & Intil & $\operatorname{sinti}$ & ( 1111 & ugrinit. & $4 \%$ & 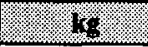 \\
\hline S95T002976 & 112:1 & DL & 1,340 & 1,470 & 1,410 & \multirow[t]{3}{*}{785} & \multirow[t]{3}{*}{34.2} & \multirow[t]{3}{*}{3.14} \\
\hline S95T002984 & $112: 2$ & DL & 483 & 492.0 & 487.4 & & & \\
\hline \$95T003255 & $114: 1$ & DL & 635 & 609.0 & 622.0 & & & \\
\hline
\end{tabular}

Table B-44. Tank 241-B-204 Analytical Results: Americium-241.

\begin{tabular}{|c|c|c|c|c|c|c|c|c|}
\hline Saniplo & Sort: & (n) & $\mathrm{P}(-1,14$ & Biplicate & Men & $40 \%$ & 的 & 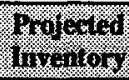 \\
\hline Hovits & \% $-11 \%$ & 1401114 & $201 / 8$ & 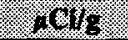 & 14 & $1 \%$ & 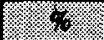 & 0 \\
\hline S95T003120 & $112: 14$ & Lower $1 / 2$ & $<0.0815$ & $<0.0851$ & $<0.0833$ & $<0.0833$ & $n / a$ & $<18.3$ \\
\hline
\end{tabular}




\begin{tabular}{|c|c|c|c|c|c|c|c|c|}
\hline$\angle \varepsilon^{\circ} 8>$ & $\mathrm{e} / \mathrm{u}$ & $08 E 0^{\circ} 0>$ & $08 \mathrm{E} 0^{\circ} 0>$ & $\left\{\angle E 0^{\circ} 0>\right.$ & $88 E 0^{\circ} 0>$ & $z_{1}$ гомо & tI:ZI I & 0ZIE0,LS6S \\
\hline (6) & $\%$ & 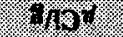 & 190\% & $8 / 8$ & 110\% & \multirow{3}{*}{ 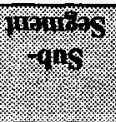 } & \multirow{3}{*}{$\begin{array}{l}\text { garagos } \\
\text { noubs }\end{array}$} & \multirow{3}{*}{$\begin{array}{l}\text { ropany. } \\
\text { qauns. }\end{array}$} \\
\hline ferom & $(2)=97$ & $10 \%$ & mon: & divitha & yory & & & \\
\hline ?2010 & ofs & 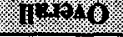 & & & : & & & \\
\hline
\end{tabular}

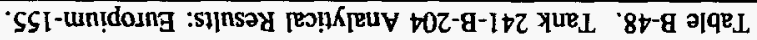

\begin{tabular}{|c|c|c|c|c|c|c|c|c|}
\hline $98^{\circ} L>$ & $E / \mathbb{U}$ & $\angle S E 0^{\circ} 0>$ & $\angle S E 0^{\circ} 0>$ & $\forall \mathcal{E E 0 ^ { \circ }} 0^{\circ}>$ & $08 \varepsilon 0^{\circ}>$ & $y_{1}$ دамо7 & tI:ZII & 0ZIE00.LS6S \\
\hline 18 & $\%$ & w & $9 \%$ & $\pi / \sigma_{0}$ & Wror & \multirow{3}{*}{$\begin{array}{l}\text { gapurass: } \\
\text {-ins }\end{array}$} & \multirow{3}{*}{$\begin{array}{l}\text { arowos } \\
\text { aroo }\end{array}$} & \multirow{3}{*}{$\begin{array}{l}\text { prominy } \\
\text { ofimis }\end{array}$} \\
\hline (3rope & (anowion & $000 \%$ & $100 \%$ & $010116 \%$ & प14 & & & \\
\hline 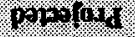 & 1081 & 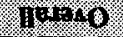 & & (:): & & & & \\
\hline
\end{tabular}

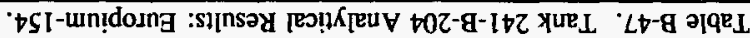

\begin{tabular}{|c|c|c|c|c|c|c|c|c|}
\hline $16^{\prime} 9>$ & $\mathrm{e} / \mathrm{u}$ & $\nabla I E O O>$ & $\nabla I E 0^{\circ} 0>$ & $\varepsilon I E 0^{\circ} 0>$ & SIE0 $0>$ & $z / 1$ IәMOT & tI:ZII & 0ZIEOOLS6S \\
\hline 10 & 4 & \% & 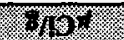 & Yhy & Why & \multirow{3}{*}{ 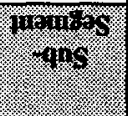 } & $103415 \%$ & \multirow{3}{*}{$6 \operatorname{mon}^{2}$} \\
\hline 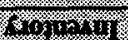 & $(1,211$ & $01 \rightarrow 3 / 4$ & $101 \%$ & 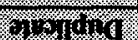 & Sy:m, & & 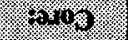 & \\
\hline $10 k^{k} \cdot 10 \%$ & (I) & 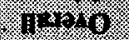 & & & & & & \\
\hline
\end{tabular}

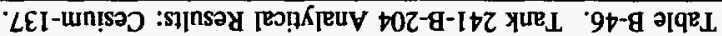

\begin{tabular}{|c|c|c|c|c|c|c|c|c|}
\hline $88^{\prime} z>$ & $\mathbf{e} / \mathbf{u}$ & IEI0 $0>$ & $1 \& 10^{\circ} 0>$ & $2110.0>$ & $6+100>$ & $z_{1} 12 \mathrm{MO}_{\mathrm{T}}$ & $\nabla I: Z I I$ & 0ZIEOOLS6S \\
\hline 8 & \% & 46 & S1 & 64 & (W) & \multirow{3}{*}{ 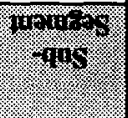 } & 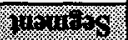 & \multirow{3}{*}{ 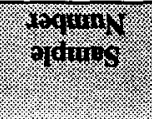 } \\
\hline 40,19119 & 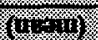 & $110 \%$ & 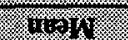 & $40 \%$ W & 111094 & & (x) & \\
\hline 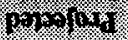 & (asti & $1040 \%$ & & & & & & \\
\hline
\end{tabular}

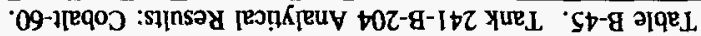


Table B-49. Tank 241-B-204 Analytical Results: Strontium-90.

\begin{tabular}{|c|c|c|c|c|c|c|c|c|}
\hline Q.iminte & (6) & 1016 & 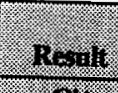 & 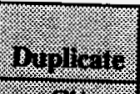 & ysin & \% & . 351 & $\sin ^{4}$ \\
\hline & 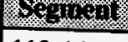 & $2-311-11$ & (s) & 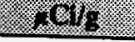 & (1) & \% & 2. & \% \\
\hline$\$ 95 T 003120$ & $112: 14$ & Lower $1 / 2$ & $4.15 E-03$ & $5.85 \mathrm{E}-03$ & $5.00 \mathrm{E}-03$ & $5.00 \mathrm{E}-030$ & 24.0 & 1.10 \\
\hline
\end{tabular}


Table B-50. Tank 241-B-204 Analytical Results: Total Alpha. (2 sheets)

\begin{tabular}{|c|c|c|c|c|c|c|c|c|}
\hline 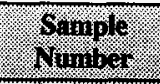 & $(4)+11 \%$ & 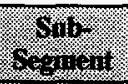 & (28) & Bing & 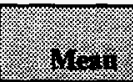 & 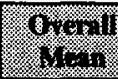 & 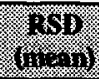 & 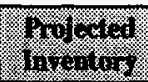 \\
\hline Yolls. & 8 & & $1 \%$ & $1 \%$ & (2) & 16. & (1) & ( \\
\hline \$95T002987 & $112: 2$ & Whole & 0.246 & 0.239 & $0.242^{1,2}$ & \multirow[t]{21}{*}{0.264} & \multirow[t]{21}{*}{7.2} & \multirow[t]{21}{*}{58.1} \\
\hline S95T003109 & $112: 3$ & Lower $1 / 2$ & 0.299 & 0.322 & $0.310^{1,2}$ & & & \\
\hline S95T003110 & $112: 4$ & Lower $1 / 2$ & 0.253 & 0.285 & $0.269^{1,3}$ & & & \\
\hline S95T003111 & $112: 5$ & Lower $1 / 2$ & 0.230 & 0.239 & $0.234^{1}$ & & & \\
\hline \$95T003112 & 112:6 & Lower $1 / 2$ & 0.178 & 0.220 & $0.199^{1,3}$ & & & \\
\hline S95T003113 & $112: 7$ & Lower $1 / 2$ & 0.287 & 0.214 & $0.251^{1,3}$ & & & \\
\hline S95T003114 & $112: 8$ & Lower $1 / 2$ & 0.214 & 0.432 & $0.323^{3}$ & & & \\
\hline S95T003115 & $112: 9$ & Lower $1 / 2$ & 0.359 & 0.324 & $0.342^{3}$ & & & \\
\hline S95T003116 & 112:10 & Lower $1 / 2$ & 0.261 & 0.279 & 0.270 & & & \\
\hline S95T003117 & $112: 11$ & Lower $1 / 2$ & 0.284 & 0.271 & 0.277 & & & \\
\hline S95T003118 & 112:12 & Lower $1 / 2$ & 0.258 & 0.268 & $0.263^{1}$ & & & \\
\hline \$95T003119 & 112:13 & Lower $1 / 2$ & 0.251 & 0.245 & 0.248 & & & \\
\hline S95T003120 & 112:14 & Lower $1 / 2$ & 0.496 & 0.419 & $0.458^{1,3}$ & & & \\
\hline S95T003334 & $114: 2$ & Lower $1 / 2$ & 0.372 & 0.323 & $0.348^{3}$ & & & \\
\hline \$95T003335 & $114: 3$ & Lower $1 / 2$ & 0.269 & 0.280 & 0.275 & & & \\
\hline S95T003336 & $114: 4$ & Lower $1 / 2$ & 0.261 & 0.282 & 0.271 & & & \\
\hline \$95T003337 & $114: 5$ & Lower $1 / 2$ & 0.194 & 0.182 & 0.188 & & & \\
\hline \$95T003338 & $114: 6$ & Lower $1 / 2$ & 0.222 & 0.182 & $0.202^{1,3}$ & & & \\
\hline S95T003339 & $114: 7$ & Lower $1 / 2$ & 0.164 & 0.174 & $0.169^{1}$ & & & \\
\hline S95T003340 & $114: 8$ & Lower $1 / 2$ & 0.204 & 0.242 & $0.223^{3}$ & & & \\
\hline S95T003341 & $114: 9$ & Lower $1 / 2$ & 0.217 & 0.236 & $0.226^{1}$ & & & \\
\hline
\end{tabular}


Table B-50. Tank 241-B-204 Analytical Results: Total Alpha. (2 sheets)

\begin{tabular}{|c|c|c|c|c|c|c|c|c|}
\hline $4 \%$ & Womis & 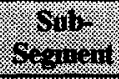 & $\mathrm{m}=\mathrm{mil}$ & 1)ialiois & 48 & W & 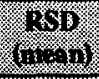 & 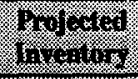 \\
\hline Solins & 8 & & or & 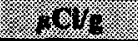 & (x) & / $/ 3$ & - & 阵 \\
\hline S95T003342 & $114: 10$ & Lower $1 / 2$ & 0.262 & 0.298 & $0.280^{3}$ & & & \\
\hline S95T003343 & $114: 11$ & Lower $1 / 2$ & 0.215 & 0.247 & $0.231^{1,3}$ & & & \\
\hline S95T003344 & $114: 12$ & Lower $1 / 2$ & 0.182 & 0.243 & $0.212^{1,3,4}$ & & & \\
\hline S95T003345 & $114: 13$ & Lower $1 / 2$ & 0.219 & 0.245 & $0.232^{1,3}$ & & & \\
\hline S95T003346 & $114: 14$ & Lower $1 / 2$ & 0.336 & 0.329 & $0.333^{1}$ & & & \\
\hline \multicolumn{3}{|c|}{ Molnow } & $24 \% 1$ & 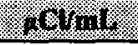 & Minu & 203111 & 8 & 4 \\
\hline \$95T002976 & 112:1 & DL & $1.36 \mathrm{E}-04$ & $1.04 \mathrm{E}-04$ & $1.19 \mathrm{E}-04^{1,2,3}$ & \multirow[t]{3}{*}{$9.98 \mathrm{E}-05$} & \multirow[t]{3}{*}{8.2} & \multirow[t]{3}{*}{$3.99 \mathrm{E}-04$} \\
\hline S95T002984 & $112: 2$ & DL & 9.75E-05 & $9.87 \mathrm{E}-05$ & $9.81 \mathrm{E}-05^{1,2}$ & & & \\
\hline S95T003255 & $114: 1$ & DL & $9.05 \mathrm{E}-05$ & $9.15 \mathrm{E}-05$ & $9.10 \mathrm{E}-05^{2}$ & & & \\
\hline
\end{tabular}

Notes:

'Spike recovery was below the quality control limit of 90 to 110 percent recovery.

${ }^{2}$ Standard recovery was below the quality control limit of 90 to 110 percent recovery.

${ }^{3} \mathrm{RPD}$ was outside the quality control limit of $\leq 10$ percent.

'Standard recovery was above the quality control limit of 90 to 110 percent recovery. 


\begin{tabular}{|c|c|c|c|c|c|c|c|}
\hline $9 \varepsilon 9 L$ & $9 S \angle L$ & $\angle S^{\circ} 9 L$ & $9 S^{\circ} 8 L$ & $(69 I-L Z) 8 L I-8 Z$ & $z_{1}$ ләмо & \multirow[b]{2}{*}{ II:ZII } & stS0E00LS6S \\
\hline$E S^{\circ} 9 L$ & $17 L L$ & $09^{\circ} 9 L$ & $22 \cdot 8 L$ & $(0 L I-L Z) 181-6 Z$ & $z_{1}$ Iadd $n$ & & ${ }_{s} E S 0 E 00 L S 6 S$ \\
\hline $8 E L L$ & $\nabla 6 \mathrm{LL}$ & $\$ 8^{\circ} 9 L$ & $20.6 L$ & $(S S I-S E)[9 I-S E$ & $z / 1$ Іәмо & \multirow[b]{2}{*}{ OI:ZII } & ¿ZSOE00LS6S \\
\hline$\varepsilon L^{\prime} 9 L$ & $98.9 L$ & $99 . \mathrm{LL}$ & $90.9 L$ & $(I E I-S E) \in E I-S E$ & $2 / 1$ Iadd $n$ & & ISOE00LS6S \\
\hline $0 L L L$ & $82: 8 L$ & $\varepsilon 6 \angle L$ & $79^{\circ} 8 \mathrm{~L}$ & $(\forall L I-L Z) 9 L I-I Z$ & z/ Iәмо7 & \multirow[b]{2}{*}{ 6:ZI I } & ऽOSOEOOLS6S \\
\hline$I Z \cdot S L$ & $8 L S L$ & $0 L^{\circ} \nabla L$ & $98^{\circ} 9 L$ & $(+11-12) 591-62$ & $z / 1$ Jadd $\Omega$ & & s6t0E00.LS6S \\
\hline $60^{\circ} Z L$ & $988 \mathrm{ZL}$ & $8 E^{\prime} 8 L$ & $\angle E^{\circ} \angle 9$ & $(S S I-6 Z) 8+I-\varepsilon Z$ & t/1 دәмот & \multirow[b]{2}{*}{ 8:ZII } & s8t0E00LS6S \\
\hline $09 . \angle L$ & $22: 8 L$ & 56.82 & $80^{\circ} \mathrm{LL}$ & $(z 8 I-\nabla z) 08 I-z z$ & $3 / \operatorname{ladd}_{\Omega}$ & & sLtOEDOLS6S \\
\hline$\varepsilon 9^{\circ} \bullet L$ & $9 \varepsilon \subseteq L$ & $S+S L$ & $9 Z^{\prime} S L$ & $(0 L I-\varepsilon Z) 09 \mathrm{I}-\nabla Z$ & Z/1 Iәмо7 & \multirow[b]{2}{*}{$L:$ ZII } & $s 9+0 \varepsilon 00 \mathrm{LS} 6 \mathrm{~S}$ \\
\hline $028 \mathrm{~L}$ & $69.8 L$ & $S Z 8 L$ & $\varepsilon 1.6 L$ & $(0 \angle I-S \mathcal{E}) \angle D I-S E$ & $2 / 1$ sadd $n$ & & ${ }_{D}$ St0E00.LS6S \\
\hline $58 \angle L$ & $\varepsilon E^{\circ} 8 L$ & $52.8 L$ & $27^{\circ} 8 L$ & $(S D I-S E)$ ISI-SE & $4 / 1$ Іәмо̆ & \multirow[b]{2}{*}{ 9:ZII } & $\$ t 0 E 00 \mathrm{LS} 6 \mathrm{~S}$ \\
\hline$I L \cdot t L$ & $2 Z^{\prime} S L$ & $06 \angle L$ & $\forall S^{\prime} Z L$ & $(\mathcal{E S I}-S \mathcal{E}) L Z I-S \mathcal{E}$ & $4 / 12 \mathrm{dd} \cap$ & & ¿Et0E00LS6S \\
\hline $76.8 \mathrm{~L}$ & $\angle S 6 L$ & $08^{\circ} 6 L$ & $S E \cdot 6 L$ & $(6 Z I-S \mathcal{E}) \mathcal{E} D I-\mathcal{S E}$ & $2 / 1$ IәMOT & \multirow[b]{2}{*}{ S:ZII } & ¿t50E00LS6S \\
\hline$E \varepsilon^{\prime} \varepsilon L$ & $10^{\circ} \circ L$ & $0 L^{\circ} 9 L$ & $Z E^{\circ} \mathrm{IL}$ & $(6 S I-S \mathcal{S})$ OII-SE & $2 / 1$ Iodd $n$ & & Itt0E00LS6S \\
\hline $\mathcal{S E} \cdot 9 L$ & $0 L 92$ & $8 L 9 L$ & $29^{\circ} 9 L$ & $(\angle O I-S E) I \angle I-S E$ & $y_{1}$ دәмо & \multirow[b]{2}{*}{$t: Z I I$} & D0t0E00.LS6S \\
\hline $5 E 8 L$ & $9 S^{\circ} 8 L$ & $1 t^{\circ} 8 \mathrm{~L}$ & $Z L^{\prime} 8 L$ & $(S S I-S E)$ SEI-SE & $4 / 1$ Iadd $\cap$ & & $.6 E 0 E 00 L S 6 S$ \\
\hline $99: \angle L$ & $\varepsilon 6 . L L$ & $\varepsilon 2.8 L$ & $29 . \angle L$ & $(I Z I-S E) \angle Z I-S E$ & z/ IәMOT & \multirow[b]{2}{*}{$\varepsilon:$ ZII } & $\$ 8 E 0 E 00 L S 6 S$ \\
\hline$E t 8 L$ & $90^{\circ} 08$ & $\angle E^{\prime} 18$ & $75.6 L$ & $(6 S I-S E) 6 S I-S E$ & $4 / 1$ Iadd $n$ & & DLEOE00LS6S \\
\hline $26{ }^{\circ} \mathrm{LL}$ & $0 I^{\circ} 6 L$ & SO $6 L$ & $S I^{\circ} 6 L$ & $(S 9 I-S E) 6 S I-S E$ & әүочM & Z:ZII & $.6262001,56 S$ \\
\hline \multicolumn{8}{|c|}{ 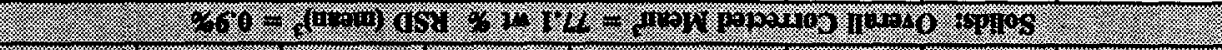 } \\
\hline 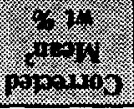 & (1) & 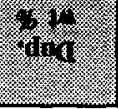 & ing. & 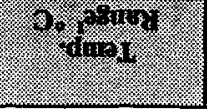 & 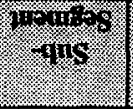 & 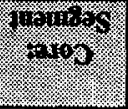 & (2) \\
\hline
\end{tabular}

(s]ə⿱丶万⿱⿰㇒一乂 


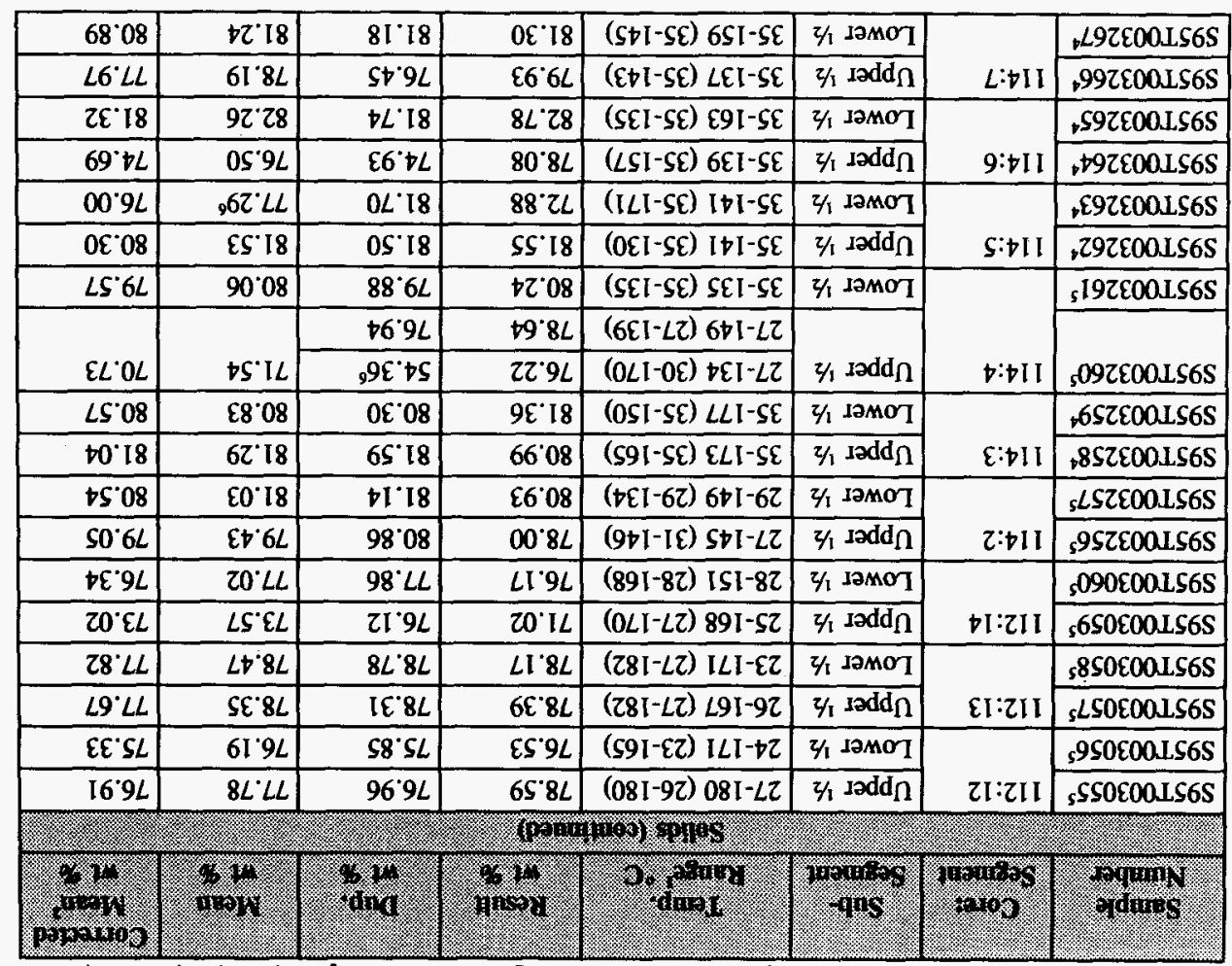

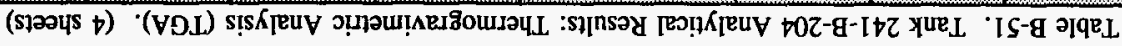


Table B-51. Tank 241-B-204 Analytical Results: Thermogravimetric Analysis (TGA). (4 sheets)

\begin{tabular}{|c|c|c|c|c|c|c|c|}
\hline 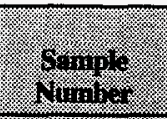 & 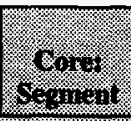 & 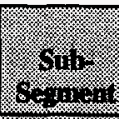 & 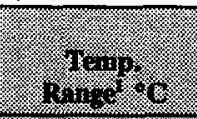 & 40 & 8 & 4 & 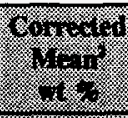 \\
\hline \multicolumn{8}{|c|}{ 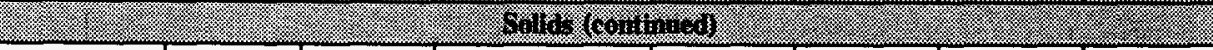 } \\
\hline S95T003268 & \multirow[t]{2}{*}{$114: 8$} & Upper $1 / 2$ & $35-157(35-120)$ & 80.15 & 79.76 & 79.96 & 79.71 \\
\hline$\$ 95 T 003269^{4}$ & & Lower $1 / 2$ & $35-160(35-137)$ & 79.78 & 79.12 & 79.45 & 79.05 \\
\hline S95T003270 & \multirow[t]{2}{*}{$114: 9$} & Upper $1 / 2$ & $35-165(35-140)$ & 80.08 & 78.54 & 79.31 & 78.86 \\
\hline S95T0032714 & & Lower $1 / 2$ & $35-155(35-119)$ & 78.59 & 78.93 & 78.76 & 78.35 \\
\hline $595 T 003272^{4}$ & \multirow[t]{2}{*}{$114: 10$} & Upper $1 / 2$ & $35-159(35-135)$ & 79.70 & 80.46 & 80.08 & 79.55 \\
\hline $5951003273^{5}$ & & Lower $1 / 2$ & $27-183(27-136)$ & 78.97 & 79.13 & 79.05 & 78.42 \\
\hline S95T003274 & \multirow[t]{2}{*}{$114: 11$} & Upper $1 / 2$ & $35-127(35-161)$ & 75.76 & 77.96 & 76.86 & 76.33 \\
\hline $595 T 003275^{4}$ & & Lower $1 / 2$ & $35-181(35-141)$ & 79.30 & 79.11 & 79.20 & 78.67 \\
\hline S95T003276 & \multirow[t]{2}{*}{ 114: 12} & Upper $1 / 2$ & $35-153(35-170)$ & 79.60 & 79.46 & 79.53 & 79.33 \\
\hline S95T003277 & & Lower $1 / 2$ & $35-167(35-163)$ & 77.87 & 78.56 & 78.22 & 77.75 \\
\hline S95T003278 & \multirow[t]{2}{*}{$114: 13$} & Upper $1 / 2$ & $\begin{array}{l}35-90(35-110) \\
{[35-110]}\end{array}$ & 50.78 & $\begin{array}{l}40.95 \\
74.58\end{array}$ & 55.44 & 54.65 \\
\hline S95T003279 & & Lower $1 / 2$ & $35-137(35-143)$ & 77.56 & 78.29 & 77.93 & 77.59 \\
\hline S95T003280 & \multirow[t]{2}{*}{$114: 14$} & Upper $1 / 2$ & $35-147(35-183)$ & 77.00 & 78.05 & 77.53 & 77.10 \\
\hline S95T0032814 & & Lower $1 / 2$ & $35-181(35-113)$ & 77.15 & 76.67 & 76.91 & 77.94 \\
\hline
\end{tabular}


Table B-51. Tank 241-B-204 Analytical Results: Thermogravimetric Analysis (TGA). (4 sheets)

\begin{tabular}{|c|c|c|c|c|c|c|c|}
\hline 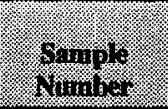 & 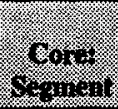 & $\frac{1}{4 y_{11}}$ & $\left(\frac{1}{10} \ln 3\right)$ & 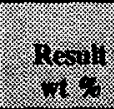 & $\frac{319}{48}$ & 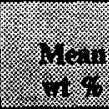 & 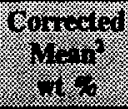 \\
\hline \multicolumn{8}{|c|}{ 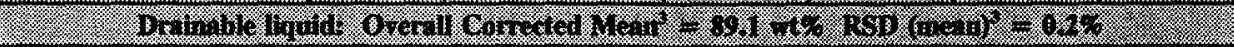 } \\
\hline S95T002976 & $112: 1$ & DL & $26-146(32-146)$ & 89.33 & 89.12 & 89.22 & 89.90 \\
\hline S95T002984 & $112: 2$ & $\mathrm{DL}$ & $29-144(31-144)$ & 89.24 & 89.33 & 89.28 & 88.77 \\
\hline S95T0032554 & $114: 1$ & DL & $35-121(35-123)$ & 89.2 & 88.56 & 88.88 & 88.88 \\
\hline
\end{tabular}

Notes:

Temp. = temperature

'The range in parentheses is for the duplicate; the range in brackets is for the triplicate.

${ }^{2}$ The mean is corrected for bydrostatic head fluid contamination based on bromide results.

${ }^{3}$ The overall corrected mean is calculated based on "Corrected Mean" results.

The percent water by thermogravimetric analysis was determined using a Mettler ${ }^{\mathrm{M}}$ instrument.

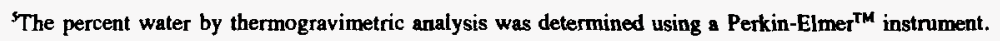

The RPD was outside the quality control limit of $\leq 10$ percent. 
Table B-52. Differential Scanning Calorimetry Results for Tank 241-B-204. (6 sheets)

\begin{tabular}{|c|c|c|c|c|c|c|}
\hline \multirow{2}{*}{ 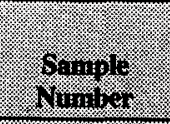 } & \multirow[b]{2}{*}{$\frac{1}{60}$} & \multirow[b]{2}{*}{$11,<-11-1$} & \multirow[b]{2}{*}{ 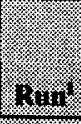 } & \multirow[b]{2}{*}{ 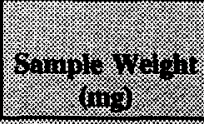 } & \multicolumn{2}{|c|}{ Hin; 10,1} \\
\hline & & & & & W. & . \\
\hline \multicolumn{7}{|c|}{8} \\
\hline \multirow[t]{2}{*}{ S95T002979² } & \multirow[t]{2}{*}{$112: 2$} & \multirow[t]{2}{*}{ Whole } & 1 & 43.122 & 97.3 & 817.7 \\
\hline & & & 2 & 18.125 & 101.3 & $1,218.0$ \\
\hline \multirow[t]{2}{*}{ S95T0030372 } & \multirow[t]{4}{*}{$112: 3$} & \multirow[t]{2}{*}{ Upper $1 / 2$} & 1 & 10.231 & 103.3 & $1,708.6$ \\
\hline & & & 2 & 35.095 & 101.3 & $1,066.1$ \\
\hline \multirow[t]{2}{*}{ S95T003038 } & & \multirow[t]{2}{*}{ Lower $1 / 2$} & 1 & 9.347 & 100.0 & $1,478.3$ \\
\hline & & & 2 & 20.201 & 99.3 & $1,368.5$ \\
\hline \multirow[t]{2}{*}{ S95T0030392 } & \multirow[t]{4}{*}{$112: 4$} & \multirow[t]{2}{*}{ Upper $1 / 2$} & 1 & 19.526 & 103.3 & $1,032.2$ \\
\hline & & & 2 & 20.134 & 103.3 & $1,292.4$ \\
\hline \multirow[t]{2}{*}{ S95T0030402 } & & \multirow[t]{2}{*}{ Lower $1 / 2$} & 1 & 26.001 & 95.3 & $1,396.6$ \\
\hline & & & 2 & 21.224 & 105.3 & $1,176.1$ \\
\hline \multirow[t]{2}{*}{ S95T0030412 } & \multirow[t]{4}{*}{$112: 5$} & \multirow[t]{2}{*}{ Upper $1 / 2$} & 1 & 19.254 & 103.3 & $1,344.7$ \\
\hline & & & 2 & 35.345 & 105.3 & 893.9 \\
\hline \multirow[t]{2}{*}{ S95T003042² } & & \multirow[t]{2}{*}{ Lower $1 / 2$} & 1 & 27.475 & 101.3 & $1,375.7$ \\
\hline & & & 2 & 21.120 & 101.3 & $1,136.3$ \\
\hline \multirow[t]{2}{*}{ S95T0030432 } & \multirow[t]{4}{*}{$112: 6$} & \multirow[t]{2}{*}{ Upper $1 / 2$} & 1 & 31.261 & 101.3 & $1,107.1$ \\
\hline & & & 2 & 30.785 & 101.3 & $1,088.6$ \\
\hline \multirow[t]{2}{*}{ S95T0030442 } & & \multirow[t]{2}{*}{ Lower $1 / 2$} & 1 & 30.841 & 97.3 & $1 ; 172.1$ \\
\hline & & & 2 & 48.951 & 97.3 & $1,049.7$ \\
\hline \multirow[t]{2}{*}{ S95T003045 } & \multirow[t]{2}{*}{$112: 7$} & \multirow[t]{2}{*}{ Upper $1 / 2$} & 1 & 17.802 & 103.3 & $1,310.7$ \\
\hline & & & 2 & 29.120 & 99.3 & $1,049.0$ \\
\hline
\end{tabular}


Table B-52. Differential Scanning Calorimetry Results for Tank 241-B-204. (6 sheets)

\begin{tabular}{|c|c|c|c|c|c|c|}
\hline \multirow{2}{*}{ 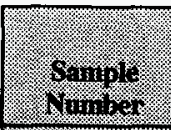 } & \multirow[b]{2}{*}{ (3) } & \multirow[b]{2}{*}{$6116-2 \cdot 110$} & \multirow[b]{2}{*}{ Bin } & \multirow[b]{2}{*}{ 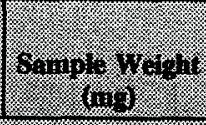 } & \multicolumn{2}{|c|}{ Jonsivilin } \\
\hline & & & & & fory. & 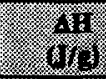 \\
\hline \multicolumn{7}{|c|}{ 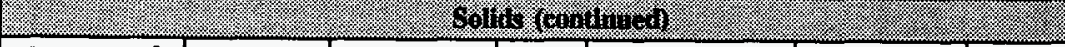 } \\
\hline \multirow[t]{2}{*}{ S95T003046 } & & \multirow[t]{2}{*}{ Lower $1 / 2$} & 1 & 28.790 & 99.3 & 905.2 \\
\hline & & & 2 & 35.459 & 97.3 & 893.2 \\
\hline \multirow[t]{2}{*}{ S95T003047 } & \multirow[t]{4}{*}{$112: 8$} & \multirow[t]{2}{*}{ Upper $1 / 2$} & 1 & 55.866 & 147.4 & 573.0 \\
\hline & & & 2 & 37.320 & 105.3 & 782.0 \\
\hline \multirow[t]{2}{*}{ S95T003048 2} & & \multirow[t]{2}{*}{ Lower $1 / 2$} & 1 & 13.610 & 115.5 & $1,756.8$ \\
\hline & & & 2 & 18.380 & 116.5 & $1,714.0$ \\
\hline \multirow[t]{2}{*}{ S95T003049 } & \multirow[t]{4}{*}{$112: 9$} & \multirow[t]{2}{*}{ Upper $1 / 2$} & 1 & 19.850 & 117.4 & $1,711.2$ \\
\hline & & & 2 & 14.010 & 116.0 & $1,694.6$ \\
\hline \multirow[t]{2}{*}{ S95T003050 } & & \multirow[t]{2}{*}{ Lower $1 / 2$} & 1 & 18.530 & 132.2 & $1,619.2$ \\
\hline & & & 2 & 16.770 & 114.8 & $1,689.6$ \\
\hline \multirow[t]{2}{*}{ S95T003051 ${ }^{2}$} & \multirow[t]{4}{*}{$112: 10$} & \multirow[t]{2}{*}{ Upper $1 / 2$} & 1 & 13.949 & 103.3 & $1,514.7$ \\
\hline & & & 2 & 23.268 & 103.3 & $1,082.5$ \\
\hline \multirow[t]{2}{*}{ S95T003052 2} & & \multirow[t]{2}{*}{ Lower $1 / 2$} & 1 & 23.447 & 99.3 & $1,119.9$ \\
\hline & & & 2 & 22.355 & 103.3 & $1,356.7$ \\
\hline \multirow[t]{2}{*}{ S95T003053 3} & \multirow[t]{4}{*}{ 112:11 } & \multirow[t]{2}{*}{ Upper $1 / 2$} & 1 & 30.220 & 128.1 & $1,636.4$ \\
\hline & & & 2 & 35.360 & 130.0 & $1,737.8$ \\
\hline \multirow[t]{2}{*}{$595 \mathrm{T003054^{3 }}$} & & \multirow[t]{2}{*}{ Lower 1/2 } & 1 & 37.160 & 124.6 & $1 ; 715.1$ \\
\hline & & & 2 & 27.750 & 112.8 & $1,763.6$ \\
\hline
\end{tabular}


Table B-52. Differential Scanning Calorimetry Results for Tank 241-B-204. (6 sheets)

\begin{tabular}{|c|c|c|c|c|c|c|}
\hline \multirow{2}{*}{ | } & \multirow[b]{2}{*}{ 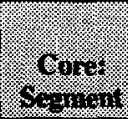 } & \multirow[b]{2}{*}{$5,11<-1.11 \%$} & \multirow[b]{2}{*}{ Ming } & \multirow[b]{2}{*}{ o. } & \multicolumn{2}{|c|}{ Toring } \\
\hline & & & & & 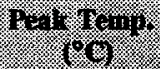 & $(x)$ \\
\hline \multicolumn{7}{|c|}{ 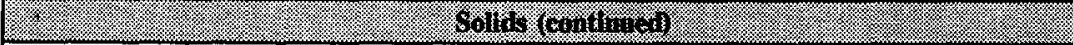 } \\
\hline \multirow[t]{2}{*}{$\$ 95 \mathrm{TO03055^{3 }}$} & \multirow[t]{2}{*}{$112: 12$} & \multirow[t]{2}{*}{ Upper $1 / 2$} & 1 & 22.570 & 118.0 & $1,531.5$ \\
\hline & & & 2 & 38.650 & 119.2 & $1,694.3$ \\
\hline \multirow[t]{2}{*}{ S95T003056 ${ }^{3}$} & & \multirow[t]{2}{*}{ Lower $1 / 2$} & 1 & 24.690 & 132.4 & $1,350.8$ \\
\hline & & & 2 & 22.870 & 116.2 & $1,452.3$ \\
\hline \multirow[t]{2}{*}{ S95T003057 $7^{3}$} & \multirow[t]{4}{*}{$112: 13$} & \multirow[t]{2}{*}{ Upper $1 / 2$} & 1 & 23.150 & 119.9 & $1,493.4$ \\
\hline & & & 2 & 27.870 & 122.0 & $1,813.7$ \\
\hline \multirow[t]{2}{*}{ S95T003058 ${ }^{3}$} & & \multirow[t]{2}{*}{ Lower $1 / 2$} & 1 & 29.390 & 124.4 & $1,758.7$ \\
\hline & & & 2 & 20.580 & 118.2 & $1,825.7$ \\
\hline \multirow[t]{2}{*}{ S95T003059 $9^{3}$} & \multirow[t]{4}{*}{$112: 14$} & \multirow[t]{2}{*}{ Upper $1 / 2$} & 1 & 22.350 & 121.6 & $1,685.1$ \\
\hline & & & 2 & 27.780 & 125.9 & $1,534.9$ \\
\hline \multirow[t]{2}{*}{ S95T003060 3} & & \multirow[t]{2}{*}{ Lower $1 / 2$} & 1 & 26.910 & 125.9 & $1,710.2$ \\
\hline & & & 2 & 26.430 & 120.1 & $1,647.8$ \\
\hline \multirow[t]{2}{*}{ S95T003256 2} & \multirow[t]{4}{*}{$114: 2$} & \multirow[t]{2}{*}{ Upper $1 / 2$} & 1 & 18.675 & 106.5 & $1,580.6$ \\
\hline & & & 2 & 24.379 & 123.0 & $1,502.7$ \\
\hline \multirow[t]{2}{*}{ S95T003257 $7^{2}$} & & \multirow[t]{2}{*}{ Lower $1 / 2$} & 1 & 12.172 & 108.3 & $1,616.4$ \\
\hline & & & 2 & 10.403 & 105.8 & $1,719.4$ \\
\hline \multirow[t]{2}{*}{ S95T003258 ${ }^{2}$} & \multirow[t]{4}{*}{$114: 3$} & \multirow[t]{2}{*}{ Upper $1 / 2$} & 1 & 8.530 & 91.8 & $1 ; 895.7$ \\
\hline & & & 2 & 10.453 & 103.3 & $1,593.6$ \\
\hline \multirow[t]{2}{*}{ S95T0032592 } & & \multirow[t]{2}{*}{ Lower $1 / 2$} & 1 & 9.050 & 103.3 & $1,830.5$ \\
\hline & & & 2 & 10.200 & 101.3 & $1,687.0$ \\
\hline
\end{tabular}


Table B-52. Differential Scanning Calorimetry Results for Tank 241-B-204. (6 sheets)

\begin{tabular}{|c|c|c|c|c|c|c|}
\hline \multirow[b]{2}{*}{ (1) } & \multirow[b]{2}{*}{$\frac{1}{104}$} & \multirow[b]{2}{*}{ 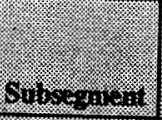 } & \multirow[b]{2}{*}{ (Ainin: } & \multirow[b]{2}{*}{ 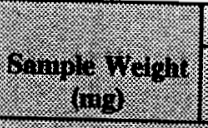 } & \multicolumn{2}{|c|}{ H. } \\
\hline & & & & & 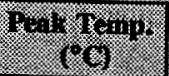 & 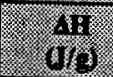 \\
\hline \multicolumn{7}{|c|}{ 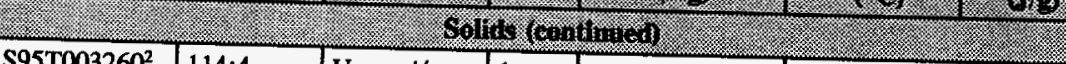 } \\
\hline \multirow[t]{2}{*}{ S95T0032602 } & \multirow[t]{4}{*}{$114: 4$} & \multirow[t]{2}{*}{ Upper $1 / 2$} & 1 & 28.915 & 99.3 & $1,172.1$ \\
\hline & & & 2 & 18.400 & 103.3 & $1,380.7$ \\
\hline \multirow[t]{2}{*}{ S95T003261 2} & & \multirow[t]{2}{*}{ Lower $1 / 2$} & 1 & 11.745 & 103.6 & $1,477.0$ \\
\hline & & & 2 & 16.001 & 103.3 & $1,429.8$ \\
\hline \multirow[t]{2}{*}{ S95T003262 } & \multirow[t]{4}{*}{$114: 5$} & \multirow[t]{2}{*}{ Upper $1 / 2$} & 1 & 15.978 & 105.3 & $1,325.5$ \\
\hline & & & 2 & 13.140 & 105.3 & $1,377.6$ \\
\hline \multirow[t]{2}{*}{ S95T0032632 } & & \multirow[t]{2}{*}{ Lower $1 / 2$} & 1 & 8.649 & 82.2 & $1,465.9$ \\
\hline & & & 2 & 11.978 & 105.3 & $1,762.9$ \\
\hline \multirow[t]{2}{*}{ S95T003264 } & \multirow[t]{4}{*}{$114: 6$} & \multirow[t]{2}{*}{ Upper $1 / 2$} & 1 & 12.452 & 101.4 & $1,649.1$ \\
\hline & & & 2 & 24.747 & 101.3 & $1,368.8$ \\
\hline \multirow[t]{2}{*}{ S95T003265 } & & \multirow[t]{2}{*}{ Lower $1 / 2$} & 1 & 12.352 & 98.0 & $1,634.5$ \\
\hline & & & 2 & 17.987 & 107.3 & $1,502.9$ \\
\hline \multirow[t]{2}{*}{ S95T003266 } & \multirow[t]{4}{*}{$114: 7$} & \multirow[t]{2}{*}{ Upper $1 / 2$} & 1 & 23.510 & 103.3 & 985.4 \\
\hline & & & 2 & 40.714 & 99.3 & 904.5 \\
\hline \multirow[t]{2}{*}{ S95T003267 } & & \multirow[t]{2}{*}{ Lower $1 / 2$} & 1 & 16.510 & 103.3 & $1,302.3$ \\
\hline & & & 2 & 19.900 & 101.3 & $1,349.4$ \\
\hline \multirow[t]{2}{*}{ S95T003268 2} & \multirow[t]{4}{*}{$114: 8$} & \multirow[t]{2}{*}{ Upper $1 / 2$} & 1 & 21.825 & $101 . \overline{3}$ & $1,275.0$ \\
\hline & & & 2 & 21.080 & 105.3 & $1,266.9$ \\
\hline \multirow[t]{2}{*}{ S95T0032692 } & & \multirow[t]{2}{*}{ Lower $1 / 2$} & 1 & 47.955 & 101.3 & 693.5 \\
\hline & & & 2 & 24.471 & 99.3 & $1,110.9$ \\
\hline
\end{tabular}


Table B-52. Differential Scanning Calorimetry Results for Tank 241-B-204. (6 sheets)

\begin{tabular}{|c|c|c|c|c|c|c|}
\hline \multirow{2}{*}{ 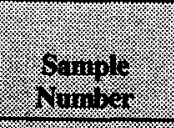 } & \multirow[b]{2}{*}{$\frac{1}{20 \%}$} & \multirow[b]{2}{*}{ 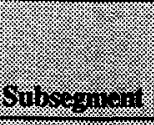 } & \multirow[b]{2}{*}{ 3in } & \multirow[b]{2}{*}{ 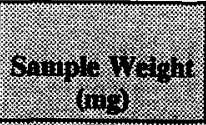 } & \multicolumn{2}{|c|}{ Hongurou } \\
\hline & & & & & 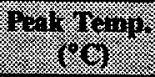 & . \\
\hline \multicolumn{7}{|c|}{ (n) } \\
\hline \multirow[t]{2}{*}{ S95T003270² } & \multirow[t]{4}{*}{$114: 9$} & \multirow[t]{2}{*}{ Upper $1 / 2$} & 1 & 13.934 & 103.3 & $1,579.4$ \\
\hline & & & 2 & 11.900 & 103.3 & $1,515.0$ \\
\hline \multirow[t]{2}{*}{ S95T003271 } & & \multirow[t]{2}{*}{ Lower $1 / 2$} & 1 & 24.020 & 103.3 & $1,209.5$ \\
\hline & & & 2 & 25.181 & 101.3 & $1,354.8$ \\
\hline \multirow[t]{2}{*}{ S95T0032722 } & \multirow[t]{4}{*}{$114: 10$} & \multirow[t]{2}{*}{ Upper $1 / 2$} & 1 & 17.950 & 105.3 & $1,154.3$ \\
\hline & & & 2 & 27.924 & 101.3 & $1,106.9$ \\
\hline \multirow[t]{2}{*}{ S95T0032732 } & & \multirow[t]{2}{*}{ Lower $1 / 2$} & 1 & 26.310 & 103.3 & 998.0 \\
\hline & & & 2 & 26.680 & 103.3 & $1,219.1$ \\
\hline \multirow[t]{2}{*}{ S95T0032742 } & \multirow[t]{4}{*}{ 114:11 } & \multirow[t]{2}{*}{ Upper $1 / 2$} & 1 & 15.835 & 105.3 & $1,554.4$ \\
\hline & & & 2 & 17.922 & 103.3 & $1,265.7$ \\
\hline \multirow[t]{2}{*}{ S95T003275 } & & \multirow[t]{2}{*}{ Lower $1 / 2$} & 1 & 40.264 & 99.3 & 915.6 \\
\hline & & & 2 & 18.840 & 103.3 & $1,139.6$ \\
\hline \multirow[t]{2}{*}{ S95T003276 } & \multirow[t]{4}{*}{$114: 12$} & \multirow[t]{2}{*}{ Upper $1 / 2$} & 1 & 34.795 & 103.3 & 965.3 \\
\hline & & & 2 & 8.670 & 107.3 & $1,675.5$ \\
\hline \multirow[t]{2}{*}{ S95T003277² } & & \multirow[t]{2}{*}{ Lower $1 / 2$} & 1 & 22.140 & 105.3 & $1,434.4$ \\
\hline & & & 2 & 20.812 & 107.3 & $1,536.1$ \\
\hline \multirow[t]{2}{*}{ S95T0032782 } & \multirow[t]{4}{*}{$114: 13$} & \multirow[t]{2}{*}{ Upper $1 / 2$} & 1 & 21.180 & 111.3 & $1,464.2$ \\
\hline & & & 2 & 7.690 & 107.6 & $1,642.2$ \\
\hline \multirow[t]{2}{*}{ S95T003279² } & & \multirow[t]{2}{*}{ Lower $1 / 2$} & 1 & 27.250 & 103.3 & $1,054.0$ \\
\hline & & & 2 & 25.140 & 103.3 & $1,166.9$ \\
\hline
\end{tabular}


Table B-52. Differential Scanning Calorimetry Results for Tank 241-B-204. (6 sheets)

\begin{tabular}{|c|c|c|c|c|c|c|}
\hline \multirow{2}{*}{ 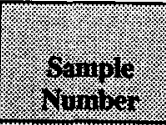 } & \multirow[b]{2}{*}{ (1) } & \multirow[b]{2}{*}{ 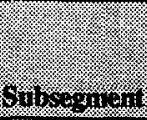 } & \multirow[b]{2}{*}{31011} & \multirow[b]{2}{*}{ ons } & \multicolumn{2}{|c|}{ Ton: } \\
\hline & & & & & 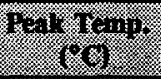 & 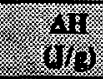 \\
\hline \multicolumn{7}{|c|}{ 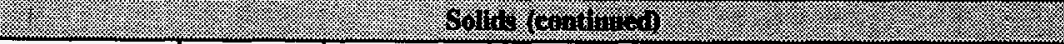 } \\
\hline \multirow[t]{2}{*}{ S95T003280 } & \multirow[t]{4}{*}{$114: 14$} & \multirow[t]{2}{*}{ Upper $1 / 2$} & 1 & 29.080 & 99.3 & $1,138.6$ \\
\hline & & & 2 & 10.880 & 101.9 & $1,616.9$ \\
\hline \multirow[t]{2}{*}{ S95T003281 } & & \multirow[t]{2}{*}{ Lower $1 / 2$} & 1 & 22.900 & 99.3 & $1,384.7$ \\
\hline & & & 2 & 11.765 & 110.8 & $1,293.4$ \\
\hline & ; & 1 & (128) & Hol & & \\
\hline \multirow[t]{2}{*}{ S95T002976 3} & \multirow[t]{2}{*}{$112: 1$} & \multirow[t]{2}{*}{ DL } & 1 & 10.560 & 111.9 & $2,051.5$ \\
\hline & & & 2 & 17.880 & 117.6 & $2,095.2$ \\
\hline \multirow[t]{2}{*}{ S95T002984 3} & \multirow[t]{2}{*}{$112: 2$} & \multirow[t]{2}{*}{ DL } & 1 & 10.390 & 102.7 & $1,985.9$ \\
\hline & & & 2 & 16.620 & 115.2 & $2,057.0$ \\
\hline \multirow[t]{2}{*}{ S95T003255² } & \multirow[t]{2}{*}{$114: 1$} & \multirow[t]{2}{*}{ DL } & 1 & 40.570 & 101.3 & 695.3 \\
\hline & & & 2 & 37.650 & 101.3 & 840.4 \\
\hline
\end{tabular}

Notes:

$\Delta \mathrm{H}=$ change in enthalpy.

${ }^{1}$ Run 1 equals the result; run 2 equals the duplicate.

${ }^{2}$ The analysis was performed on Mettler ${ }^{\mathrm{TM}}$ equipment.

${ }^{3}$ The analysis was performed on Perkin-Elmer ${ }^{\mathrm{TM}}$ equipment. 


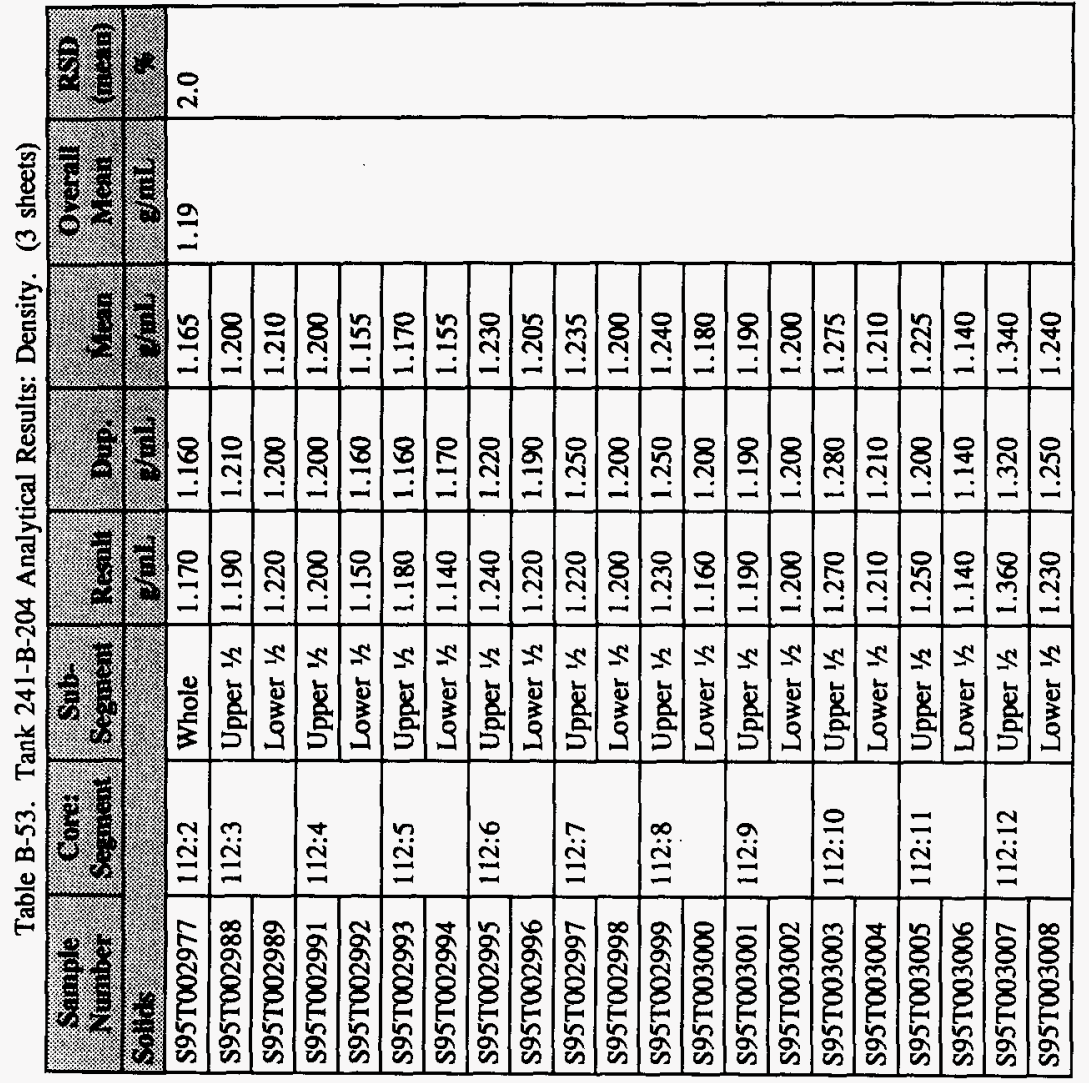




\begin{tabular}{|c|c|c|c|c|c|c|c|}
\hline & & $59 I^{\circ} \mathrm{I}$ & OSI $\mathrm{I}$ & $08 I^{\prime} \mathrm{I}$ & $2 / 1$ Iәмо T & \multirow[b]{2}{*}{$6: t I I$} & LIZEOOLS6S \\
\hline & & $0 \angle I^{\circ} I$ & $091 . \mathrm{I}$ & $08 \mathrm{I}^{\circ} \mathrm{I}$ & $\mathrm{z} / 1$ Iadd $\Omega$ & & $912 \varepsilon 00 \mathrm{LS} 6 \mathrm{~S}$ \\
\hline & & $S L I \cdot 1$ & $0 L I \cdot I$ & $08 I^{-1}$ & \% IOMOT & & SIZEOaLS6S \\
\hline & & $S \angle I^{\circ} I$ & $09 I^{\prime} 1$ & $06 \mathrm{I}^{\circ} \mathrm{I}$ & $z / 1$ ladd $\Omega$ & $8: t I I$ & tIZE00LS6S \\
\hline & & S9I. & OSI I & $08 \mathrm{I} \cdot \mathrm{I}$ & $2 / \mathrm{I}$ IอMOT & & EIZE00LS6S \\
\hline & & S9I'I & $09 I^{\circ} \mathrm{I}$ & $0 L I \cdot I$ & $z_{1}$ Ioddn & $L: D I I$ & ZIZE00LS6S \\
\hline & & $108 \mathrm{I}^{\circ} \mathrm{I}$ & $0 \not \tau \cdot 1$ & $021 \div \mathrm{I}$ & $z / 1$ LAMOT & & IIZE00LS6S \\
\hline & & $s+\mathrm{I}^{\circ} \mathrm{l}$ & $001 . \mathrm{I}$ & $06 \mathrm{I}^{\circ} \mathrm{I}$ & $z_{1}$ saddn & 9:011 & 0IZع00LS6S \\
\hline & & $08 I^{\circ} \mathrm{I}$ & $09 I^{\prime} I$ & $002 \cdot I$ & $2 / 1$ เәмо & & $60 Z \varepsilon 00 \perp S 6 S$ \\
\hline & & $091^{\circ} 1$ & 021.1 & $002 \div$ & $z_{1}$ Iadd $n$ & s:tII & 80ZE00LS6S \\
\hline & & $0 \mathrm{LII}$ & 061.1 & OSI'I & $2 / 1$ JOMOT & & $\angle 0 Z \varepsilon 00 \mathrm{LS} 6 \mathrm{~S}$ \\
\hline & & OSI ${ }^{\circ}$ & OSIT & OSI'I & $z_{1}$ Ladd $\Omega$ & $t: t I I$ & $90 z \varepsilon 00 \mathrm{LS} 6 \mathrm{~S}$ \\
\hline & & $091^{\circ} 1$ & 091.1 & $091 \%$ & Z/, samo7 & & S0zE00IS6S \\
\hline & & $0 \angle I^{\circ} \mathrm{I}$ & 061.1 & OSI'I & $z_{1}$ ladd $\Pi$ & $\varepsilon: \downarrow I I$ & t02E00LS6S \\
\hline & & $09 I^{\circ} \mathrm{I}$ & OLI'I & OSI'I & $2 / 1$ sәм07 & & E0zE00IS6S \\
\hline & & $581^{\circ} \mathrm{T}$ & OLI' I & 002.1 & z/1 Iaddn & $Z: t I I$ & zozE00LS6S \\
\hline & & $08 E^{\circ} \mathrm{I}$ & O8E. I & $08 E^{\circ} \mathrm{I}$ & $y_{1}$ ग्MO & & 2IOEOOLS6S \\
\hline & & $581^{\circ} \mathrm{I}$ & $081^{\circ} \mathrm{I}$ & $06 I^{*} \mathrm{I}$ & $z_{1} \operatorname{sadd} \Omega$ & $\rightarrow I: Z I I$ & $110600156 S$ \\
\hline & & $0 \angle 2 \cdot 1$ & $08 \mathrm{Z}^{\circ} \mathrm{I}$ & $092^{\circ} \mathrm{I}$ & $y_{1}$ IOMO & & $010600156 \mathrm{~S}$ \\
\hline & & S92'I & $09 Z^{\circ} I$ & $0 L Z^{\circ} I$ & $z_{1}$ Iadd $\Omega$ & EI:ZII & $600 E 00 \mathrm{LS} 6 \mathrm{~S}$ \\
\hline 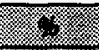 & $91 \%$ & 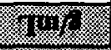 & 10096 & Tyif & 1: & & 84100 \\
\hline$\frac{194 \%}{10 \%}$ & 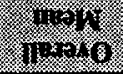 & (19) & 49 & 10 & 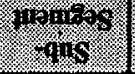 & 10, & 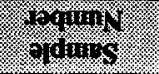 \\
\hline
\end{tabular}

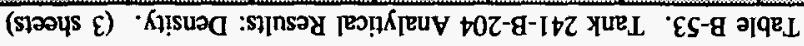




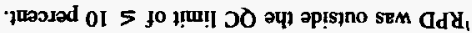

:əุoN

\begin{tabular}{|c|c|c|c|c|c|c|c|}
\hline \multirow[b]{3}{*}{ s.0 } & \multirow[b]{3}{*}{ SO ${ }^{\circ} \mathrm{I}$} & $S+0 . I$ & 090 I & $0 E 0^{\circ} \mathrm{I}$ & 70 & $I: t I I$ & SSZEO0JLS6S \\
\hline & & SSO I & $090^{\circ} \mathrm{I}$ & $0 S 0^{\circ} \mathrm{I}$ & $\mathrm{TO}$ & 2:ZII & $\$ 86200 \mathrm{LS} 6 \mathrm{~S}$ \\
\hline & & SSO'I & $0 S 0^{\circ} \mathrm{I}$ & $090^{\circ} \mathrm{I}$ & 7a & I:ZII & 9L6200LS6S \\
\hline \multirow[t]{11}{*}{8.} & torart & $611 \%$ & W14\% & why & & \multicolumn{2}{|c|}{ 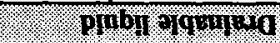 } \\
\hline & & $00 Z^{*} \mathrm{I}$ & $022 * I$ & $08 \mathrm{I}^{\prime} \mathrm{I}$ & $z / 1$ JәMOT & \multirow[b]{2}{*}{ DI:tII } & LZZEOOLS6S \\
\hline & & S6I'I & $00 Z^{\circ} I$ & $06 \mathrm{I}^{\circ} \mathrm{I}$ & $z_{1}$ Iədd $_{\Omega}$ & & 9ZZE0OLS6S \\
\hline & & $\operatorname{SIZ} I$ & $0 t 2 \cdot 1$ & $06 \mathrm{I}^{\circ} \mathrm{I}$ & $z_{1}$ IวMO & \multirow[b]{2}{*}{$\varepsilon I: t I I$} & SZZEOOLS6S \\
\hline & & $091^{\circ} \mathrm{I}$ & OEI I & $061^{\circ} 1$ & $\psi_{1}$ sedd $\bigcap$ & & tZZE00LS6S \\
\hline & & SSI'I & $0 S I^{*} \mathrm{I}$ & $09 I^{\prime} \mathrm{I}$ & $\psi / 1$ IәMOT & \multirow[b]{2}{*}{ ZI:tII } & EZ2E0ALS6S \\
\hline & & $06 \mathrm{I}^{\circ} \mathrm{I}$ & $06 \mathrm{I}^{\circ} \mathrm{I}$ & $06 \mathrm{I}^{\circ} \mathrm{I}$ & $2 / 1 \operatorname{Iodd}_{\Omega}$ & & ZZZE00LS6S \\
\hline & & $0 \angle I I$ & $0 \angle I^{\circ} I$ & $0 \angle I^{\circ} \mathrm{I}$ & 3/1 гомот & \multirow[b]{2}{*}{$I I: \mapsto I I$} & IZZE00LS6S \\
\hline & & $091^{\circ} \mathrm{I}$ & $08 I^{\circ} \mathrm{I}$ & $00 I^{\circ} \mathrm{I}$ & $y / i$ Iadd $n$ & & 0Z2E00LS6S \\
\hline & & OLI'I & $091^{\circ} 1$ & $08 I^{\circ} \mathrm{I}$ & $4 / 1$ เәмо & \multirow[b]{2}{*}{ OI:tII } & 6IZE00LS6S \\
\hline & & $\mathrm{SSI}^{\circ} \mathrm{I}$ & $09 \mathrm{I}^{\circ} \mathrm{I}$ & $0 S I^{\circ} 1$ & $\psi_{i}$ Iadd $\Omega$ & & 8IZEOOLS6S \\
\hline 8 . & (1) & $11 \%$ & $H^{10} \%$ & 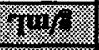 & & & (5) \\
\hline$(1+6+1)$ & 11186 & 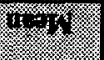 & 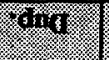 & 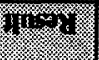 & $9=18+10 \%$ & 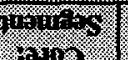 & 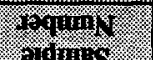 \\
\hline$(\ln 36$ & Hxpers & & & & silus & $x^{2}$ & $60 \operatorname{sen} 8$ \\
\hline
\end{tabular}

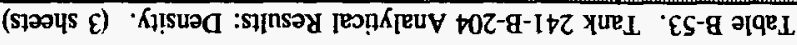




\begin{abstract}
APPENDIX C
RESULTS OF HYDROSTATIC HEAD FLUID CONTAMINATION CHECK FOR SINGLE-SHELL TANK 241-B-204
\end{abstract}


WHC-SD-WM-ER-581 Rev. 0

This page intentionally left blank. 


\section{APPENDIX C}

\section{RESULTS OF HYDROSTATIC HEAD FLUID CONTAMINATION CHECK FOR SINGLE-SHELL TANK 241-B-204}

\section{C.1 INTRODUCTION AND ANALYTE TABLE DESCRIPTION}

Appendix C reports the results of the HHF contamination check for the 1995 core sampling and analysis event. Lithium and bromide were measured to detect contamination of the waste samples by the HHF. Lithium was determined by ICP; bromide by ion chromatography. Because lithium may form insoluble compounds with the tank waste matrix, the HHF corrections to the percent water values were based on the bromide results.

Column 1 lists the laboratory sample identification number for each analyte. For sampling rationale, locations, and a description of the sampling event, see Section 3.0 and Appendix A.

Column 2 lists the core and segment from which each sample was derived. The first number is the core number; it is followed by a colon and the segment number.

Column 3 lists the name of the segment portion (subsegment) from which the sample was taken. This can be the entire segment (whole), the drainable liquid portion (DL), or the upper or lower half segment portions.

Columns 4 and 5 (Resuit and Duplicate) are self-explanatory.

Column 6 (Mean) is the average of the result and duplicate values. All values, including those below the detection level (indicated by the less-than symbol, <), were averaged in calculating the sample means. If the result and duplicate values were both nondetected, the mean is expressed as a nondetected value. If one of the two values is nondetected and one is detected, or if both are detected, then the sample mean is reported as a detected value. The result and duplicate values and the result/duplicate means, are reported in the tables exactly as found in the original laboratory data package. The means may appear to have been rounded up in some cases and rounded down in others. This is because the analytical results given in the tables may have fewer significant figures than originally reported, not because the means were incorrectly calculated.

The four quality control parameters assessed on the tank 241-B-204 samples were standard recoveries, spike recoveries, duplicate analyses (RPDs), and blanks. These were summarized in Section 5.1.2. Sample and duplicate pairs, in which any of the quality control parameters were outside their specified limits, are footnoted in column 6 of the following tables. 


\begin{tabular}{|c|c|c|c|c|c|}
\hline$S L I Z$ & 0622 & $09^{\circ} 02$ & $2 / 1$ IOMOI & & S8ZE00LS6S \\
\hline$S L^{\prime} S I$ & $0 S \varsigma I$ & 00.91 & $2 / \pi$ Iadd $\Omega$ & $\varepsilon: t I I$ & †8ZE00LS6S \\
\hline $08^{\circ}+\varepsilon$ & $O Z^{\circ} S E$ & $0 t^{\circ}+\varepsilon$ & $z_{1}$ IวMOT & \multirow[b]{2}{*}{$\tau: t I I$} & E8ZE00LS6S \\
\hline$S \varepsilon^{\circ} 0 Z$ & $0 I^{\circ} 0 Z$ & $09^{\circ} 02$ & $z / \mathrm{r}$ radd $\Omega$ & & Z8ZE00IS6S \\
\hline $560 t$ & $0 t^{\circ} 6 \varepsilon$ & $0 S^{\circ} z \phi$ & $\%$ IวMOT & \multirow[b]{2}{*}{$\nabla I: Z I I$} & $780 E 00 \mathrm{LS} 6 \mathrm{~S}$ \\
\hline $\mathcal{S E} 9 \mathcal{E}$ & $08^{\circ} I E$ & 06.00 & $2 / \pi$ Iodd $n$ & & ع80E00LS6S \\
\hline $0 \angle \angle b$ & $0<96$ & $0 L^{\prime} 8 t$ & $4 / \mathrm{I} I \mathrm{MOT}$ & \multirow[b]{2}{*}{ EI:ZII } & 280E00IS6S \\
\hline st.9s & $06 \angle 5$ & $00^{\circ} \subseteq \varsigma$ & $4 / 1$ sadd $\Omega$ & & I80E00LS6S \\
\hline $06^{\circ} \varepsilon 9$ & $05^{\circ} 09$ & $O E^{*} \angle 9$ & $z / 12 \mathrm{MOT}$ & \multirow[b]{2}{*}{ ZI:ZII } & 080E00LS6S \\
\hline S5:08 & $08^{\circ} 08$ & $0 \varepsilon^{\circ} 08$ & $\psi_{1} \operatorname{Iedd}_{\Omega}$ & & 6LOEOOLS6S \\
\hline S8.It & $0 \angle L^{\prime} O t$ & $00^{\circ} \varepsilon t$ & $z / 1$ IOMOT & \multirow[b]{2}{*}{ II:ZII } & 8L0E00LS6S \\
\hline$O S^{\circ} \angle Z$ & $00 \angle 2$ & $05^{\circ} 92$ & $2 / 1 \operatorname{Iadd}_{\Omega}$ & & $\angle L O E 00 L S 6 S$ \\
\hline $50^{\circ}$ It & $0 S^{\circ}[t$ & $09^{\circ} 0$ & \% IəMO & \multirow[b]{2}{*}{ 0I:ZII } & 9LOEOOLS6S \\
\hline$S 0^{\circ} \star \varepsilon$ & $0^{\circ} \mathrm{SE}$ & $\mathrm{OI}^{\circ} \varepsilon \mathcal{E}$ & $2 / \mathrm{I} \operatorname{Iddd}_{\Omega}$ & & SLOEOOLS6S \\
\hline $09^{\circ}+\varepsilon$ & $0 t^{\circ}+\varepsilon$ & $08^{\circ} \nabla \varepsilon$ & $4 / 1$ IOMO & \multirow[b]{2}{*}{ 6:ZII } & DLOEOOLS6S \\
\hline$S L \cdot 9 E$ & $0 t^{\circ} 9 \mathcal{E}$ & $0 \mathrm{I}^{\circ} L \varepsilon$ & $\%$ Iadd $\Omega$ & & ELOEO0LS6S \\
\hline$s \tau^{\circ} \bullet s$ & $0 \varepsilon^{\circ} \triangleright \varsigma$ & $0 z^{\circ} \nabla s$ & $2 / r$ IәMOT & \multirow[b]{2}{*}{$8: Z I I$} & ZLOEOOLS6S \\
\hline $0 L \angle \varepsilon$ & $0 z \cdot 8 \mathcal{E}$ & $0 z^{\circ} \angle \varepsilon$ & $y_{1} \operatorname{radd}_{\Omega}$ & & ILOEOOLS6S \\
\hline $08^{\circ} 8 t$ & 0586 & $0 I^{\circ} 6 t$ & $2 / 1$ IOMOT & \multirow[b]{2}{*}{$L: Z I I$} & 0LOEOOLS6S \\
\hline${ }_{2} S S^{\circ} Z \mathcal{E}$ & $0 \tau \cdot \angle \varepsilon$ & $06 \%$ & $z_{1}$ I2dd $n$ & & 690800LS6S \\
\hline 50.82 & $0 I^{-9} 92$ & $00^{\circ} 0 \varepsilon$ & $4 / \mathrm{I}$ IOMOI & \multirow[b]{2}{*}{ 9:ZII } & $890 E 00 L 56 \mathrm{~S}$ \\
\hline $\mathcal{S S}^{\cdot}$ IE & $0 I^{\circ} z \varepsilon$ & $\infty 0^{\circ} I \varepsilon$ & $2 / 1$ Iadd $n$ & & L90E00IS6S \\
\hline${ }_{\mathrm{I}} \$ 9^{\circ} 9 \mathcal{E}$ & $0 S^{\prime} \nabla \varepsilon$ & $08 \cdot 8 \varepsilon$ & L/ IOMOT & \multirow[b]{2}{*}{ s:ZII } & 990E00LS6S \\
\hline$s t \cdot 2 s$ & $02 \cdot 25$ & $0 L^{\prime} Z S$ & $z_{1}$ radd $\Omega$ & & \$90E00LS6S \\
\hline $0 Z \angle I$ & $0 Z^{\circ} \angle I$ & $0 Z^{\circ} \angle I$ & \% Iәмо 1 & \multirow[b]{2}{*}{ D:ZI I } & $\$ 90 E 00 \mathrm{LS6S}$ \\
\hline${ }_{2} \mathrm{SS}^{\circ} 8 \mathrm{I}$ & $0 z \cdot 12$ & 06 SI & $2 / 1$ Iadd & & $\varepsilon 90 \varepsilon 00 \mathrm{LS} 6 \mathrm{~S}$ \\
\hline $06^{\circ} \mathrm{EI}$ & $O E^{*} t I$ & $0 S^{\circ} \varepsilon I$ & \% IәMOI & \multirow[b]{2}{*}{$\varepsilon: Z I I$} & 290E00LS6S \\
\hline$S E^{-I Z}$ & $09^{\circ} \mathrm{IZ}$ & $0 I^{\circ} \mathrm{IZ}$ & $2 / \mathrm{radd} \Omega$ & & I90E00LS6S \\
\hline${ }_{t} S^{\prime} E O I$ & $0^{\circ}$ SOI & $0.20 I$ & ว०पМ & Z:ZII & 086200LS6S \\
\hline Whit: & Mr & Wh & & \multicolumn{2}{|c|}{ (1) } \\
\hline 40311 & agerdira & 109028 & 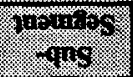 & 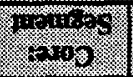 & 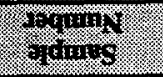 \\
\hline
\end{tabular}

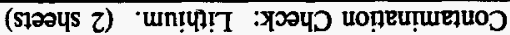

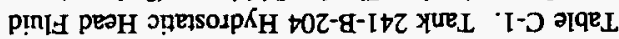


WHC-SD-WM-ER-581 Rev. 0

Table C-1. Tank 241-B-204 Hydrostatic Head Fluid

Contamination Check: Lithium. (2 sheets)

\begin{tabular}{|c|c|c|c|c|c|}
\hline 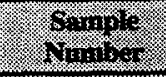 & $x^{2}$ & 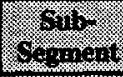 & 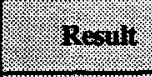 & Buplicale & Menit \\
\hline Somor. & . & & 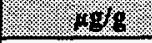 & 1488 & 3. \\
\hline S95T003286 & \multirow[t]{2}{*}{$114: 4$} & Upper $1 / 2$ & 56.00 & 60.40 & 58.20 \\
\hline S95T003287 & & Lower $1 / 2$ & 29.10 & 30.20 & 29.65 \\
\hline S95T003288 & \multirow[t]{2}{*}{$114: 5$} & Upper $1 / 2$ & 108.0 & 102.0 & 105.0 \\
\hline S95T003289 & & Lower $1 / 2$ & 61.30 & 61.80 & 61.55 \\
\hline S95T003290 & \multirow[t]{2}{*}{$114: 6$} & Upper $1 / 2$ & 107.0 & 103.0 & 105.0 \\
\hline \$95T003291 & & Lower $1 / 2$ & 38.40 & 37.60 & 38.00 \\
\hline S95T003292 & \multirow[t]{2}{*}{$114: 7$} & Upper $1 / 2$ & 15.70 & 18.00 & 16.85 \\
\hline S95T003293 & & Lower $1 / 2$ & 17.20 & 16.20 & 16.70 \\
\hline S95T003294 & \multirow[t]{2}{*}{$114: 8$} & Upper $1 / 2$ & 23.20 & 24.00 & 23.60 \\
\hline S95T003295 & & Lower $1 / 2$ & 24.00 & 27.00 & 25.50 \\
\hline S95T003296 & \multirow[t]{2}{*}{$114: 9$} & Upper $1 / 2$ & 40.20 & 37.40 & 38.80 \\
\hline S95T003297 & & Lower $1 / 2$ & 22.80 & 22.10 & 22.45 \\
\hline S95T003298 & \multirow[t]{2}{*}{$114: 10$} & Upper $1 / 2$ & 56.60 & 50.70 & 53.65 \\
\hline \$95T003299 & & Lower $1 / 2$ & 48.10 & 48.60 & 48.35 \\
\hline S95T003300 & \multirow[t]{2}{*}{$114: 11$} & Upper $1 / 2$ & 36.20 & 37.40 & 36.80 \\
\hline S95T003301 & & Lower $1 / 2$ & 39.40 & 38.30 & 38.85 \\
\hline S95T003302 & \multirow[t]{2}{*}{$114: 12$} & Upper $1 / 2$ & 12.80 & 16.00 & $14.40^{2}$ \\
\hline S95T003303 & & Lower $1 / 2$ & 41.10 & 42.60 & 41.85 \\
\hline S95T003304 & \multirow[t]{2}{*}{$114: 13$} & Upper $1 / 2$ & 23.30 & 21.20 & 22.25 \\
\hline S95T003305 & & Lower $1 / 2$ & 12.10 & 12.90 & 12.50 \\
\hline S95T003306 & \multirow[t]{2}{*}{$114: 14$} & Upper $1 / 2$ & 24.00 & 22.80 & 23.40 \\
\hline S95T003307 & & Lower $1 / 2$ & 17.50 & 17.90 & 17.70 \\
\hline \multicolumn{3}{|c|}{ 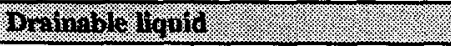 } & $10 \% \mathrm{mi}$ & $24 m \mathrm{H}$ & $64 \% 11$ \\
\hline S95T002976 & 112:1 & $\mathrm{DL}$ & $<2.01$ & $<2.01$ & $<2.01$ \\
\hline S95T002984 & $112: 2$ & $\mathrm{DL}$ & 126.0 & 124.0 & 125.0 \\
\hline S95T003255 & $114: 1$ & DL & 4.160 & 4.020 & 4.090 \\
\hline
\end{tabular}

Notes:

'The spike recovery was below the quality control limit of 85 to 115 percent recovery.

'The RPD was outside the quality control limit of $\leq 15$ percent. 
Table C-2. Tank 241-B-204 Hydrostatic Head

Fluid Contamination Check: Bromide. (2 sheets)

\begin{tabular}{|c|c|c|c|c|c|}
\hline 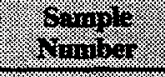 & carts. & Seventir & inemill & Buplicate & Merm \\
\hline Sotid: & (3) & (2) & 8.861 & 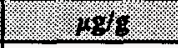 & $\begin{array}{r}488 \\
\end{array}$ \\
\hline S95T002981 & $112: 2$ & Whole & 1,510 & 1,730 & 1,620 \\
\hline S95T003085 & \multirow[t]{2}{*}{$112: 3$} & Upper $1 / 2$ & 2,780 & 2,900 & 2,840 \\
\hline S95T003086 & & Lower $1 / 2$ & 359.9 & 377.0 & 368.4 \\
\hline S95T003087 & \multirow[t]{2}{*}{$112: 4$} & Upper $1 / 2$ & 275.9 & 316.0 & 295.9 \\
\hline S95T003088 & & Lower $1 / 2$ & 443.0 & 442.0 & 442.5 \\
\hline S95T003089 & \multirow[t]{2}{*}{$112: 5$} & Upper $1 / 2$ & 751.3 & 764.0 & 757.6 \\
\hline S95T003090 & & Lower $1 / 2$ & 983.7 & 850.0 & 916.9 \\
\hline S95T003091 & \multirow[t]{2}{*}{$112: 6$} & Upper $1 / 2$ & 619.0 & 578.0 & 598.6 \\
\hline S95T003092 & & Lower $1 / 2$ & 664.1 & 678.0 & 671.0 \\
\hline S95T003093 & \multirow[t]{2}{*}{$112: 7$} & Upper $1 / 2$ & 653.0 & 721.0 & 687.0 \\
\hline S95T003094 & & Lower $1 / 2$ & 862.5 & 856.0 & 859.2 \\
\hline S95T003095 & \multirow[t]{2}{*}{$112: 8$} & Upper $1 / 2$ & 817.2 & 842.0 & 829.6 \\
\hline S95T003096 & & Lower $1 / 2$ & 795.4 & 860.0 & 827.7 \\
\hline S95T003097 & \multirow[t]{2}{*}{$112: 9$} & Upper $1 / 2$ & 696.2 & 679.0 & 687.6 \\
\hline S95T003098 & & Lower $1 / 2$ & 803.9 & 777.0 & 790.5 \\
\hline S95T003099 & \multirow[t]{2}{*}{$112: 10$} & Upper $1 / 2$ & 173.7 & 174.0 & 173.8 \\
\hline S95T003100 & & Lower $1 / 2$ & 751.1 & 743.0 & 747.0 \\
\hline S95T003101 & \multirow[t]{2}{*}{$112: 11$} & Upper $1 / 2$ & 1,100 & 1,130 & 1,120 \\
\hline S95T003102 & & Lower $1 / 2$ & 1,510 & 1,530 & 1,520 \\
\hline S95T003103 & \multirow[t]{2}{*}{$112: 12$} & Upper $1 / 2$ & 1,130 & 1,130 & 1,130 \\
\hline S95T003104 & & Lower $1 / 2$ & 1,020 & 1,060 & 1,040 \\
\hline S95T003105 & \multirow[t]{2}{*}{$112: 13$} & Upper $1 / 2$ & 929.2 & 914.0 & 921.6 \\
\hline S95T003106 & & Lower $1 / 2$ & 920.2 & 863.0 & 891.6 \\
\hline S95T003107 & \multirow[t]{2}{*}{$112: 14$} & Upper $1 / 2$ & 599.8 & 593.0 & 596.4 \\
\hline S95T003108 & & Lower $1 / 2$ & 861.7 & 862.0 & 861.9 \\
\hline S95T003308 & \multirow[t]{2}{*}{$114: 2$} & Upper $1 / 2$ & 521.9 & 569.0 & 545.5 \\
\hline S95T003309 & & Lower $1 / 2$ & 813.7 & 734.0 & 773.9 \\
\hline S95T003310 & \multirow[t]{2}{*}{$114: 3$} & Upper $1 / 2$ & 404.9 & 406.0 & 405.4 \\
\hline S95T003311 & & Lower $1 / 2$ & 409.5 & 420.0 & 414.8 \\
\hline
\end{tabular}


Table C-2. Tank 241-B-204 Hydrostatic Head

Fluid Contamination Check: Bromide. (2 sheets)

\begin{tabular}{|c|c|c|c|c|c|}
\hline ofingle & 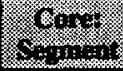 & $80+m=1$ & Pesint & Bupllow & Mesin \\
\hline 80165 & (3) & 8 & (2) & 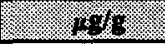 & 40 \\
\hline S95T003312 & \multirow[t]{2}{*}{$114: 4$} & Upper $1 / 2$ & 815.7 & 812.0 & 813.9 \\
\hline S95T003313 & & Lower $1 / 2$ & 742.9 & 736.0 & 739.5 \\
\hline S95T003314 & \multirow[t]{2}{*}{$114: 5$} & Upper $1 / 2$ & 1,950 & 1,890 & 1,920 \\
\hline S95T003315 & & Lower $1 / 2$ & 1,630 & 1,580 & 1,610 \\
\hline S95T003316 & \multirow[t]{2}{*}{$114: 6$} & Upper $1 / 2$ & 2,080 & 2,150 & 2,120 \\
\hline S95T003317 & & Lower $1 / 2$ & 1,580 & 1,510 & 1,550 \\
\hline S95T003318 & \multirow[t]{2}{*}{$114: 7$} & Upper $1 / 2$ & 294.0 & 315.0 & 304.5 \\
\hline S95T003319 & & Lower $1 / 2$ & 567.7 & 564.0 & 565.9 \\
\hline S95T003320 & \multirow[t]{2}{*}{$114: 8$} & Upper $1 / 2$ & 377.6 & 379.0 & 378.3 \\
\hline S95T003321 & & Lower $1 / 2$ & 588.7 & 585.0 & 586.9 \\
\hline S95T003322 & \multirow[t]{2}{*}{$114: 9$} & Upper $1 / 2$ & 651.1 & 659.0 & 655.0 \\
\hline S95T003323 & & Lower $1 / 2$ & 580.7 & 568.0 & 574.4 \\
\hline S95T003324 & \multirow[t]{2}{*}{$114: 10$} & Upper $1 / 2$ & 786.5 & 802.0 & 794.2 \\
\hline S95T003325 & & Lower $1 / 2$ & 881.8 & 884.0 & 882.9 \\
\hline S95T003326 & \multirow[t]{2}{*}{$114: 11$} & Upper $1 / 2$ & 644.7 & 698.0 & 671.4 \\
\hline S95T003327 & & Lower $1 / 2$ & 741.5 & 770.0 & 755.8 \\
\hline S95T003328 & \multirow[t]{2}{*}{$114: 12$} & Upper $1 / 2$ & 297.7 & 300.0 & 298.9 \\
\hline S95T003329 & & Lower $1 / 2$ & 647.6 & 627.0 & 637.3 \\
\hline S95T003330 & \multirow[t]{2}{*}{$114: 13$} & Upper $1 / 2$ & 497.8 & 490.0 & 493.9 \\
\hline S95T003331 & & Lower $1 / 2$ & 459.9 & 460.0 & 459.9 \\
\hline s95T003332 & \multirow[t]{2}{*}{$114: 14$} & Upper $1 / 2$ & 552.4 & 589.0 & 507.7 \\
\hline S95T003333 & & Lower $1 / 2$ & 580.4 & 622.0 & 601.2 \\
\hline \multicolumn{3}{|c|}{ 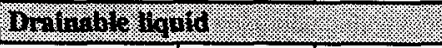 } & $4.8 \mathrm{gmi}$ & 18.mi: & $1804 \mathrm{~min}$ \\
\hline S95T002976 & $112: 1$ & DL & $<267$ & 1060 & 664 \\
\hline S95T002984 & $112: 2$ & $\mathrm{DL}$ & 1,680 & 1,710 & 1,690 \\
\hline S95T003255 & 114:1 & $\mathrm{DL}$ & $<267$ & $<267$ & $<226$ \\
\hline
\end{tabular}


WHC-SD-WM-ER-581 Rev. 0

This page intentionally left blank. 
WHC-SD-WM-ER-581 Rev. 0

APPENDIX D

HISTORICAL SAMPLING RESULTS

D-1 
WHC-SD-WM-ER-581 Rev. 0

This page intentionally left blank. 


\section{APPENDIX D}

\section{HISTORICAL SAMPLING RESULTS}

\section{D.1 INTRODUCTION}

Table D-1 lists the analytical results from a historical sampling event performed in 1978. The sample, reference number 1974, was reported as black in color with a consistency of soft grease. No additional specifics were available from historical records. These historical data are not validated and are included for comparison only; decisions affecting the waste in tank 241-B-204 should not be based on these data.

Table D-1. Historical Sampling Results for Tank 241-B-204. ${ }^{1}$

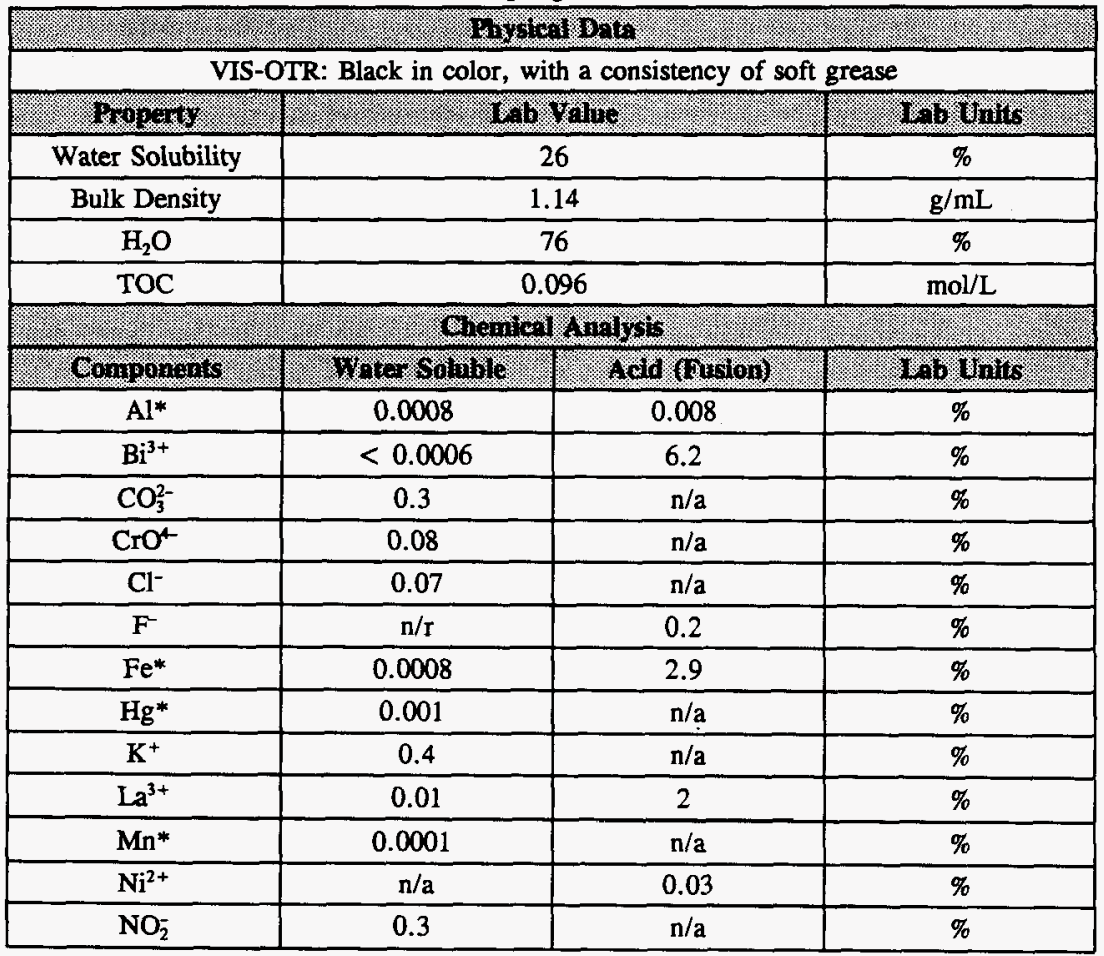


WHC-SD-WM-ER-581 Rev. 0

\begin{tabular}{|c|c|c|c|}
\hline \multicolumn{4}{|c|}{ 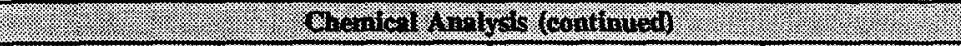 } \\
\hline 8omboncin: & (X) & icid (nision) & Yas units \\
\hline $\mathrm{NO}_{3}^{-}$ & 2.8 & $<0.07$ & $\%$ \\
\hline $\mathrm{Na}^{+}$ & 1.4 & $\mathrm{n} / \mathrm{a}$ & $\%$ \\
\hline $\mathrm{OH}^{-}$ & 0.1 & $\mathrm{n} / \mathrm{a}$ & $\%$ \\
\hline $\mathrm{PO}_{4}^{3-}$ & 0.1 & 1.2 & $\%$ \\
\hline $\mathrm{SO}_{4}^{2-}$ & $<0.05$ & $<0.1$ & $\%$ \\
\hline $\mathrm{SiO}_{2}^{2-}$ & 0.004 & 0.3 & $\%$ \\
\hline 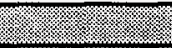 & \multicolumn{2}{|c|}{ 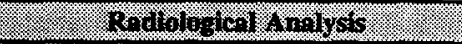 } & (1) \\
\hline 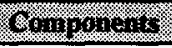 & (6) & igngurous & $(8), 3 / 115$ \\
\hline $\mathrm{U}^{*}$ & 4.63E-08 & $9.71 \mathrm{E}-06$ & $g / g$ \\
\hline $\mathrm{Pu}^{*}$ & $1.16 \mathrm{E}-09$ & $9.74 \mathrm{E}-06$ & $g / g$ \\
\hline $\mathrm{Am}^{*}$ & $n / r$ & $\mathrm{n} / \mathrm{a}$ & $\mathrm{n} / \mathrm{a}$ \\
\hline${ }^{89} \mathrm{Sr} \mathrm{Sr}$ & 0.004 & 4 & $\mu \mathrm{Ci} / \mathrm{g}$ \\
\hline${ }^{137} \mathrm{Cs}$ & 0.005 & 0.007 & $\mu \mathrm{Ci} / \mathrm{g}$ \\
\hline${ }^{155} \mathrm{Eu}^{*}$ & $n / r$ & $n / a$ & $\mathrm{n} / \mathrm{a}$ \\
\hline
\end{tabular}

Notes:

* = All oxidation states

$\mathbf{n} / \mathbf{a}=$ not applicable or not avaiłable

$\mathrm{n} / \mathbf{r}=$ Analysis not requested

${ }^{1}$ Horton (1978) 


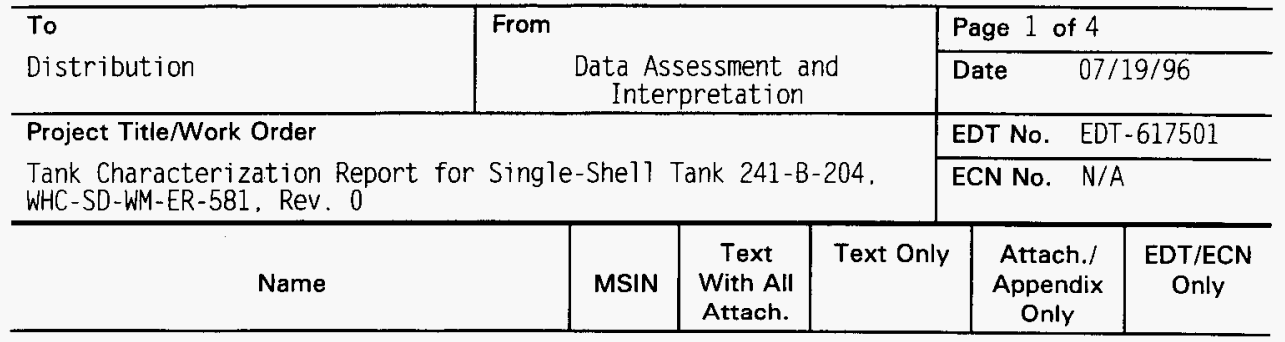

\section{OFFSITE}

Sandia National Laboratory

P.0. Box 5800

MS-0744, Dept. 6404

Albuquerque, NM 87815

D. Powers

Nuclear Consulting Services Inc.

P. 0. Box 29151

Columbus, $\mathrm{OH}$ 43229-01051

J. L. Kovach

Chemical Reaction Sub-TAP

P.0. Box 271

Lindsborg. KS 67456

B. C. Hudson

$x$

Tank Characterization Panel

Senior Technical Consultant

Contech

7309 Indian School Road

Albuquerque, NM 87110

J. Arvisu

$x$

U. S. Department of Energy - Headquarters

Office of EnvironmentaT Restoration and Waste Management EM-563 12800 Middlebrook Road

Germantown. MD 20874

J. A. Poppitti

$x$

Jacobs Enqineering Group

B5-36

$x$ 
DISTRIBUTION SHEET

\begin{tabular}{|c|c|c|c|c|c|}
\hline To & \multirow{2}{*}{\multicolumn{3}{|c|}{$\begin{array}{c}\text { Data As } \\
\text { Inter }\end{array}$}} & \multicolumn{2}{|l|}{ Page 2 of 4} \\
\hline Distribution & & & & \multicolumn{2}{|c|}{$07 / 19 / 96$} \\
\hline \multicolumn{4}{|l|}{ Project Title/Work Order } & \multicolumn{2}{|c|}{ EDT No. $\quad$ EDT-617501 } \\
\hline \multicolumn{4}{|c|}{$\begin{array}{l}\text { Tank Characterization Report for Single-Shell Tank 241-B-204, } \\
\text { WHC-SD-WM-ER-581. Rev. } 0\end{array}$} & ECN No. $\quad \mathrm{N} / \mathrm{f}$ & \\
\hline Name & MSIN & $\begin{array}{c}\text { Text } \\
\text { With All } \\
\text { Attach. }\end{array}$ & Text Only & $\begin{array}{l}\text { Attach./ } \\
\text { Appendix } \\
\text { Only }\end{array}$ & $\begin{array}{l}\text { EDT/ECN } \\
\text { Only }\end{array}$ \\
\hline
\end{tabular}

SAIC

20300 Century Boulevard. Suite 200-B

Germantown, MO 20874

H. Sutter

555 Quince Orchard Rd. . Suite 500

Gaithersburg. MD 20878

P. Szerszen

$x$

Los Alamos Laboratory

CST-14 MS-J586

P. 0 . Box 1663

Los Alamos, NM 87545

S. F. Agnew (4)

$x$

Los Alamos Technical Associates

T. T. Tran

B1-44 X

Oqden Environmental

101 East Wellsian Way

Richland. WA 99352

R. J. Anema

$x$

$\mathrm{CH} 2 \mathrm{M} \mathrm{Hill}$

P. O. BOX 91500

Bél levue. WA 98009-2050

M. McAfee

$x$

Tank Advisory Panel

102 Windham Road

Oak Ridge. TN 37830

D. O. Campbe17

$X$ 


\section{DISTRIBUTION SHEET}

\begin{tabular}{|c|c|c|c|c|c|}
\hline To & \multirow{2}{*}{\multicolumn{3}{|c|}{$\begin{array}{l}\text { From } \\
\text { Data Assessment and } \\
\text { Interpretation }\end{array}$}} & \multicolumn{2}{|l|}{ Page 3 of 4} \\
\hline Distribution & & & & Date & $9 / 96$ \\
\hline \multicolumn{4}{|l|}{ Project Title/Work Order } & \multicolumn{2}{|c|}{ EDT No. EDT-617501 } \\
\hline \multicolumn{4}{|c|}{$\begin{array}{l}\text { Tank Characterization Report for Single-Shel1 Tank 241-B-204, } \\
\text { WHC-SD-WM-ER-581. Rev. } 0\end{array}$} & \multicolumn{2}{|l|}{ ECN No. $\quad \mathrm{N} / \mathrm{A}$} \\
\hline Name & MSIN & $\begin{array}{l}\text { Text } \\
\text { With All } \\
\text { Attach. }\end{array}$ & Text Only & $\begin{array}{l}\text { Attach./ } \\
\text { Appendix } \\
\text { Only }\end{array}$ & $\begin{array}{c}\text { EDT/ECN } \\
\text { Only }\end{array}$ \\
\hline
\end{tabular}

ONSITE

Department of Enerqy - Richland Operations

J. F. Thompson

W. S. Liou

S7.54 $\quad x$

N. W. Willis

$57.54 \quad x$

S7.54 $x$

ICF-Kaiser Hanford Company

R. L. Newe 11

S3-09 $x$

Pacific Northwest Laboratory

N. G. Colton

K3-75 X

X J. R. Gormsen

K7 -28

K5-12

S. A. Hartley

K $7-94$

J. G. Hill

G. J. Lumetta

P7 -25

A. F. Noonan

K9-81

Westinghouse Hanford Company

H. Babad

D. A. Barnes

G. R. B $700 m$

W. L. Cowley

L. A. Diaz

G. L. Dunford

E. J. Eberlein

D. B. Engelman

J. S. Garfield

* J. D. Guberski

D. L. Herting

D. C. Hetzer

G. Jansen

G. D. Johnson

T. J. Kelley

N. W. Kirch

M. J. Kupfer

J. E. Meacham
S7-14

R1-80

H5-61

A3-37

T6-06

S7-81

R2-12

R1-49

H5-49

R2-06

T6-09

S6-31

H6-33

S7 -15

S7 -21

R2-11

H5 - 49

$57-15$
$X$

$X$

* Aduanced Dist Dkas 


\section{DISTRIBUTION SHEET}

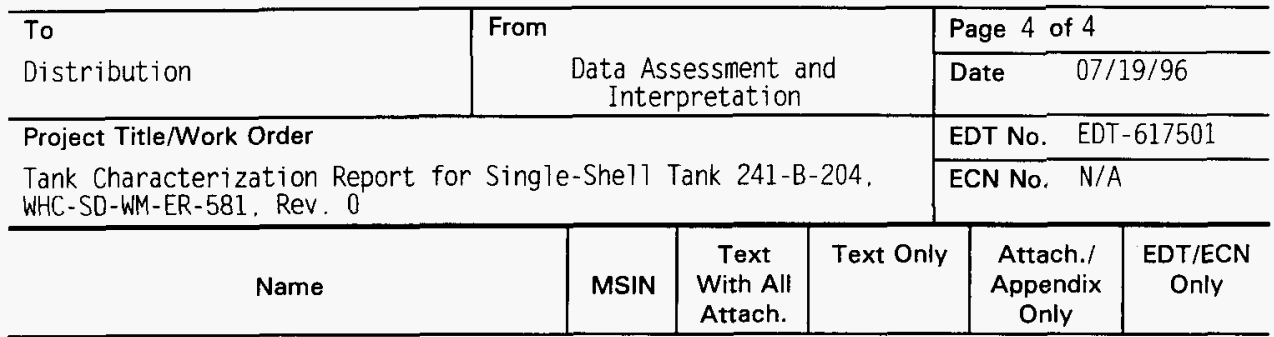

Westinghouse Hanford Company continued

W. C. Miller

C. T. Narquis

D. E. Place

D. A. Reynolds

L. M. Sasaki (2)

L. W. Shelton, Jr.

B. C. Simpson

G. L. Troyer

L. R. Webb

K. A. White

IfIC (Fank Farm Information Center)

Central Files

EDMC

ERC (Environmental Resource Center) OSTI $(2)$

TCRC (10)

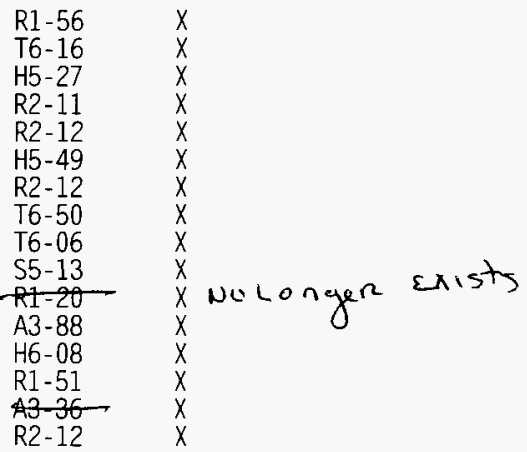

